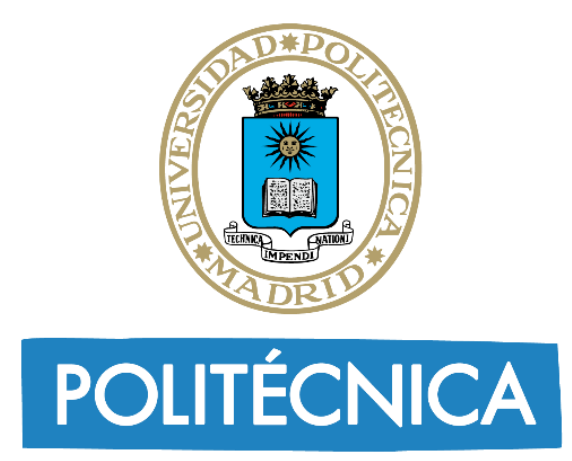

Universidad Politécnica de Madrid

Escuela Técnica Superior de Ingenieros Industriales

\title{
Estudio de la distribución espacial de aerosoles y gases traza en la atmósfera de Madrid mediante la técnica DOAS
}

Tesis Doctoral

David García Nieto, MSc.

Director:

Prof. Alfonso Saiz López

2020 


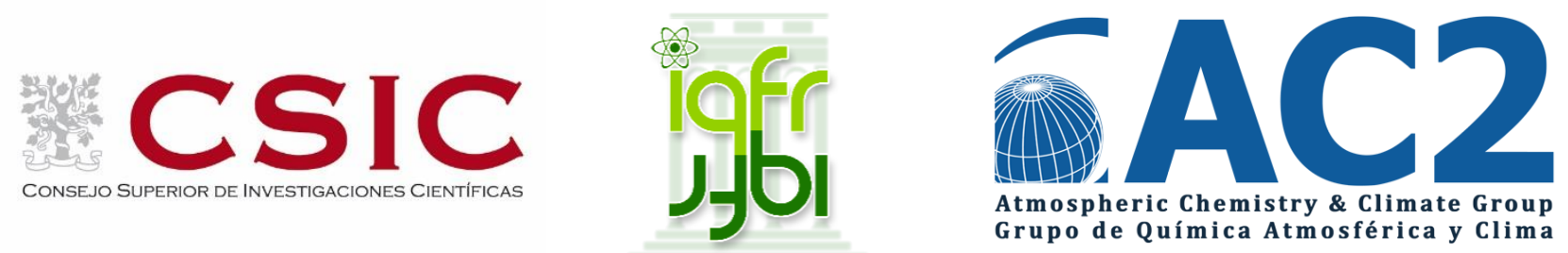

Instituto de Química Física Rocasolano (CSIC)

Grupo de Química Atmosférica y Clima

\title{
Estudio de la distribución espacial de aerosoles y gases traza en la atmósfera de Madrid mediante la técnica DOAS
}

\author{
Tesis Doctoral \\ David García Nieto, MSc.
}

Director:

Prof. Alfonso Saiz López

2020 

Título:

Estudio de la distribución espacial de aerosoles y gases traza en la atmósfera de Madrid mediante la técnica DOAS

Autor:

David García Nieto, MSc.

Supervisor:

Prof. Alfonso Saiz López.

Tribunal nombrado por el Mgfco. Y Excmo. Sr. Rector de la Universidad Politécnica de Madrid, el día de de 2020
Presidente
Vocal D.
Vocal D.
Vocal D.
Secretario D.
Suplente D.
Suplente D.

Realizado el acto de defensa y lectura de la Tesis Doctoral el de de 2020.

Calificación:
El presidente
El secretario
Los vocales 

A Paqui, José, Alba, Loli y Pepe, sin su cariño y apoyo incondicional en mis peores momentos, nada de esto hubiera sido posible 



\section{Agradecimientos}

En primer lugar querría agradecer todo este trabajo a mi director, Prof. Alfonso Saiz López, por su ayuda, guía y confianza durante todos estos años. Su exigencia ha representado en todo momento una ocasión para continuar aprendiendo y mejorando. Pero sobre todo me gustaría agradecerle por la oportunidad que me dio para poder llevar a cabo mi tesis doctoral y vivir una gran experiencia.

También querría agradecer a mi tutor, Prof. Rafael Borge, por su continua e inestimable ayuda, tanto administrativa como científica, en todos estos años, así como el apoyo y la confianza dados.

Por otra parte, durante la tesis he tenido la oportunidad de poder hacer campañas de medida y viajes que representaron, todos ellos, una experiencia muy valiosa e inolvidable. Gracias a toda la gente que hizo posible la campaña CINDI-2 (en Holanda, donde pude compartir buenas experiencias con mis compañeras del INTA: Olga, Mónica, Cristina y Margarita), así como los cruceros científicos PEACETIME (especial agradecimiento a Cécile Gieu y a Karine Desbouefs) y ARAON. Asímismo me gustaría aprovechar la oportunidad de agradecer al CfA de Boston los dos meses que pude pasar allí, y de manera especial al Dr. Gonzalo González Abad y al Prof. Kelly Chance por su ayuda y el inmejorable trato recibido. Pude aprender mucho de ellos.

En cuanto a la parte instrumental, el desarrollo de los instrumentos MAXDOAS -y por tanto las medidas espectrales- no hubiera sido posible sin la ayuda de Nacho y José, del taller mecánico. Gracias a ellos también.

A lo largo de todos estos años, han sido muchos los compañeros de grupo que he tenido, con todos ellos (los que estuvieron y los que aún continúan) pasé muy buenos 
momentos: María, Manolo, David, Nando, Leti, Nani, Alba, Caterina, Carlos, Mónica, Paul, Vega, Noemí, Dani, Jesús, Qinyi, Ángel, Roberto, Federico, Javi, Rafa, Shiney, Anoop. Gracias por los recuerdos.

Quería dejar una mención aparte para Nuria, con quien he podido compartir experiencias increíbles, geniales e irrepetibles, y sin cuyo apoyo no hubiera podido llegar siquiera a escribir esto. He aprendido mucho de ella, y siempre que me caí me ayudó a levantarme. Nunca podré llegar a agradecerte suficiente, mil gracias por todo.

Por último, muchísimas gracias a mis padres Paqui y José, a mi hermana Alba y a mis tíos Loli y Pepe por su gran ayuda y apoyo en todo momento. Siempre me han querido y enseñado mucho, y en todo momento han representado mi refugio en mis momentos más difíciles. Un último y afectuoso recuerdo a mi abuelo Sebastián, quien hubiera estado muy orgulloso de haber podido llegar a verme en este momento.

A todos, muchas gracias. 


\section{Table of contents}

\section{Abstract}

Resumen

1 INTRODUCTION.

.31

1.1 Motivation..............................................................................................

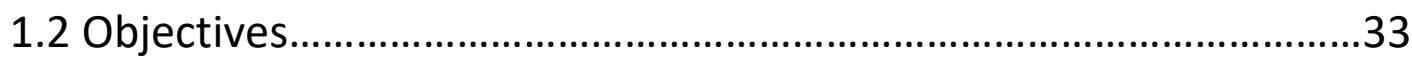

2 ATMOSPHERE OF THE EARTH........................................................35

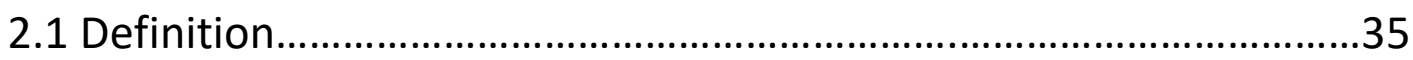

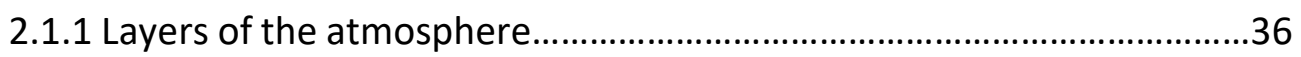

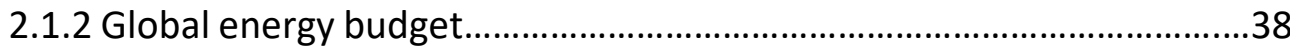

2.2 Composition of the atmosphere.........................................................40

2.2.1 Main atmospheric trace compounds........................................................41

2.3 The greenhouse effect.....................................................................42

2.4 Properties of light through the atmosphere........................................45

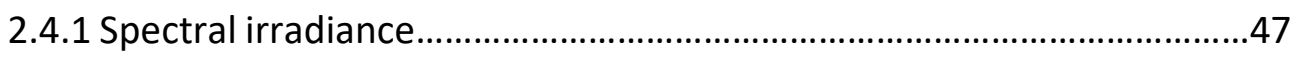

2.4.2 Solar radiation at the surface of the Earth.................................................47

2.5 Atmospheric effects.........................................................................48

2.5.1 Absorption and scattering effects in the atmosphere.............................48

3 FUNDAMENTALS OF DOAS............................................................51

3.1 The Beer-Lambert Law.........................................................................52 


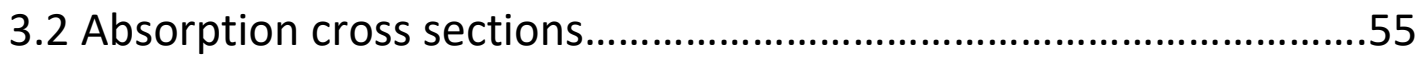

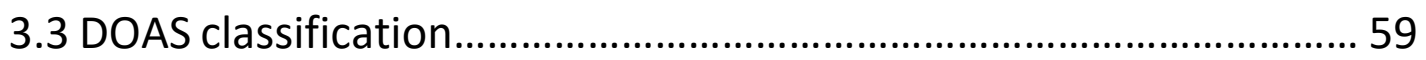

3.4 Multi AXis Differential Optical Absorption Spectroscopy...................60

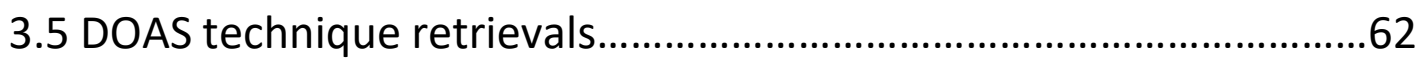

3.6 Instrumental slit function ..................................................................65

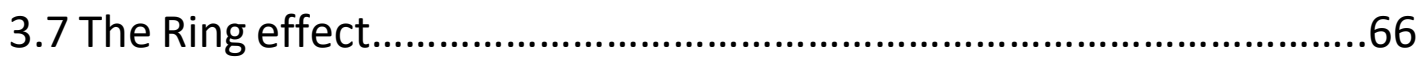

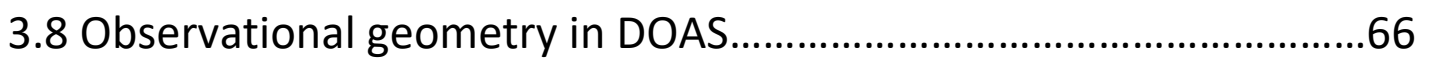

3.8.1 Solar Azimuth Angle................................................................................6

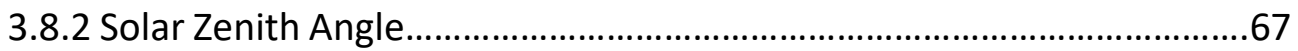

3.8.3 Viewing Azimuth Angle..........................................................................67

3.8.4 Viewing Elevation Angle.......................................................................67

3.8.5 Air mass factor........................................................................................68

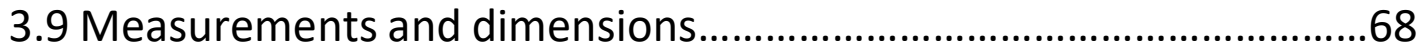

4 RADIATIVE TRANSFER..........................................................71

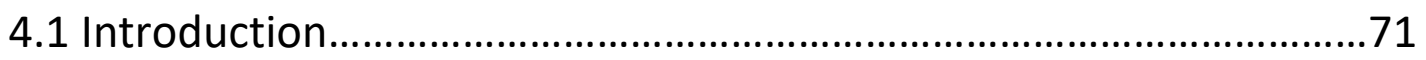

4.2 The forward model and its matrix notation......................................73

4.3 Aerosol extinction and trace gas profile retrieval..............................76

4.3.1 A priori profiles.........................................................................................

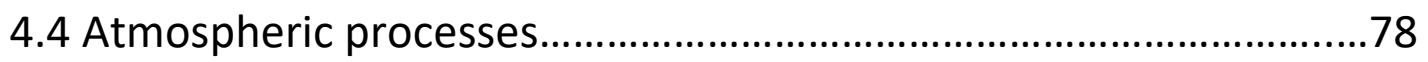

4.4.1 In-scattering and out-scattering..........................................................78

4.4.2 Rayleigh scattering.................................................................................

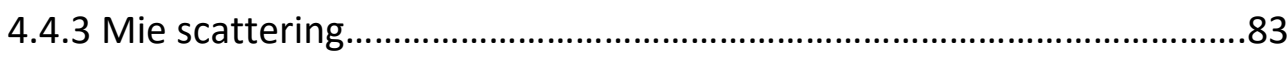

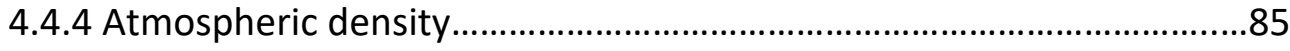

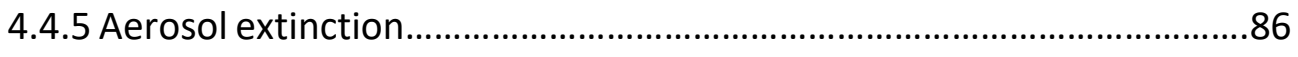

4.4.6 Integrating over light paths........................................................................86

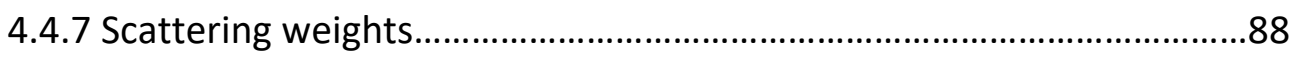


4.4.8 Multiple scattering and albedo.........................................................90

4.5 Cloud screening....................................................................91

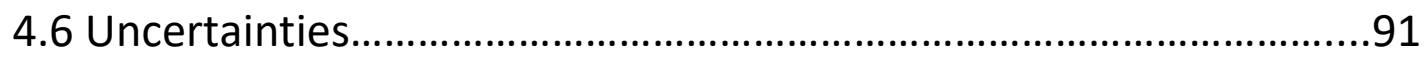

4.7 Detection limit and quality filters...........................................92

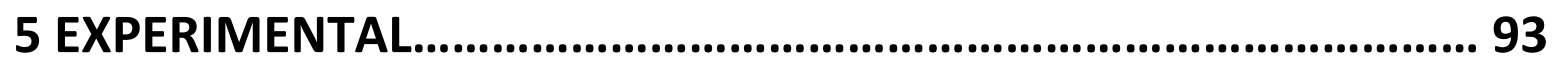

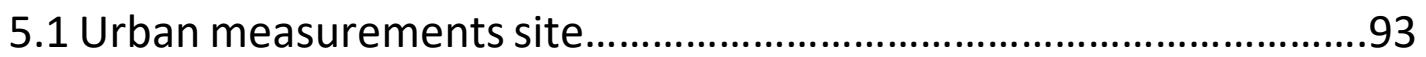

5.2 Description of the MAXDOAS-1D instrument.....................................94

5.2.1 Instrument control software...............................................96

5.3 Development of a new MAXDOAS-2D instrument.............................96

5.3.1 Measurements setup........................................................98

5.3.2 Alignment procedure............................................................99

5.4 Common parameters and instrumental effects...............................100

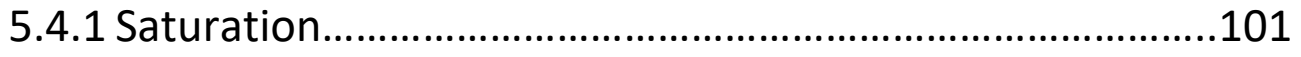

5.4.2 Electronic offset...............................................................101

5.4.3 Dark current......................................................................102

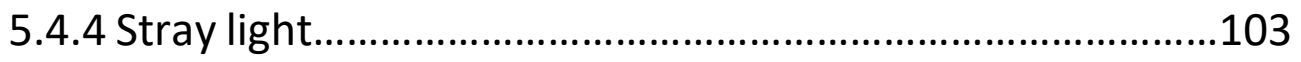

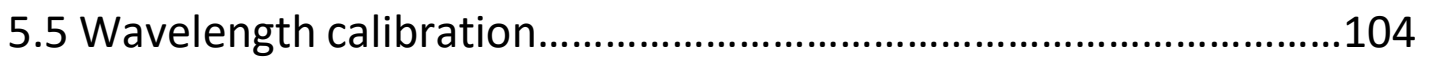

$6 \mathrm{NO}_{2}$ AND HONO............................................................107

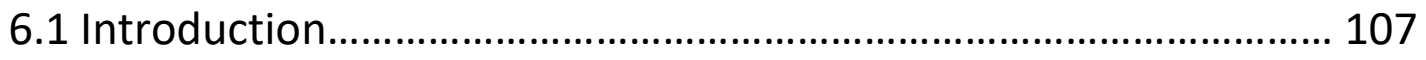

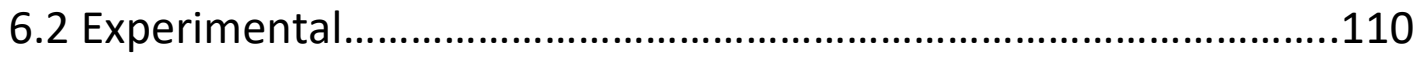

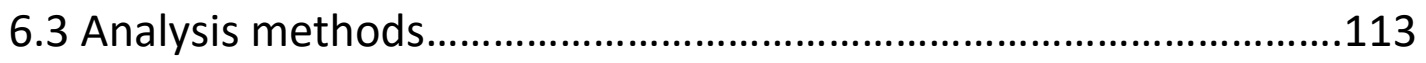

6.3.1 Cloud screening and quality filters.....................................................113

6.3.2 Spectral detection..............................................................................113 
6.3.3 Inversion parameters........................................................................114

6.4 Results and discussion...................................................................114

6.4.1 $\mathrm{O}_{4}$ DSCDs correction factor...............................................................114

6.4.2 Aerosols extinction and vertical profiles.................................................116

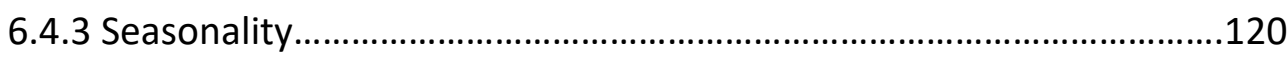

6.4.4 Estimation of $\mathrm{OH}$ production from HONO photolysis............................125

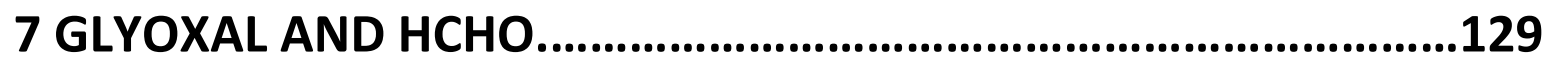

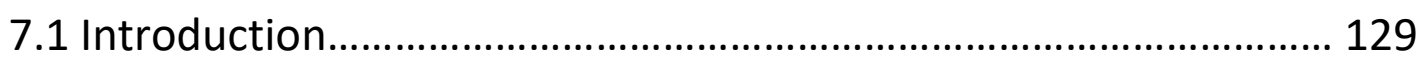

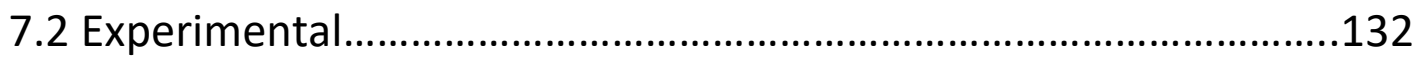

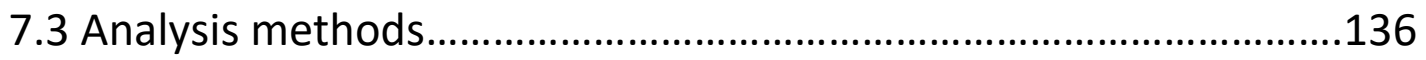

7.3.1 Cloud screening and quality filters.........................................................136

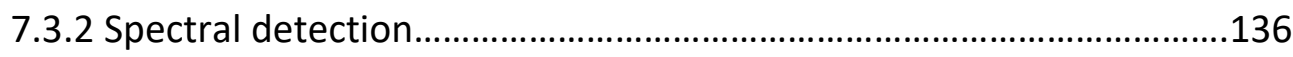

7.3.3 Profile retrieval configuration.................................................................137

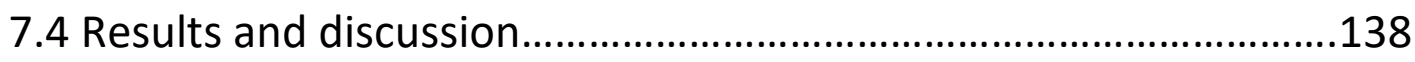

7.4.1 HCHO profiles....................................................................................138

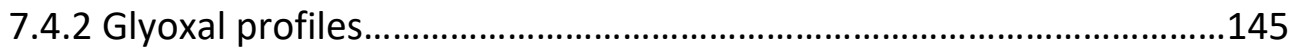

7.5 The glyoxal/formaldehyde ratio.......................................................147

\section{DEVELOPMENT OF A NEW MAXDOAS-2D INSTRUMENT..............151}

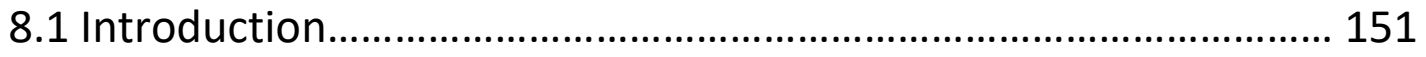

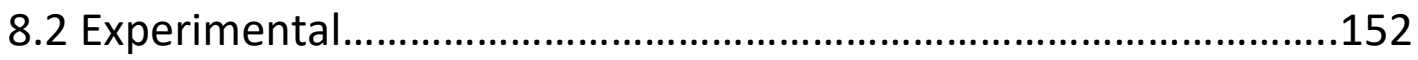

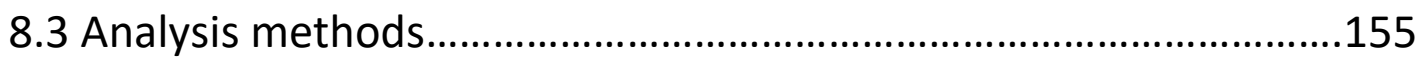

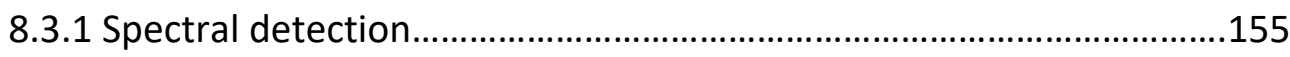

8.3.2 Inversion algorithm and vertical profiles..............................................155

8.3.3 Estimation of $\mathrm{NO}_{2}$ gradients..............................................................161

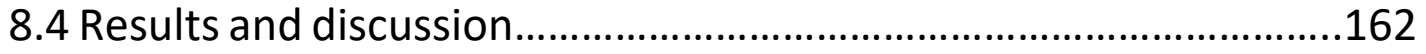




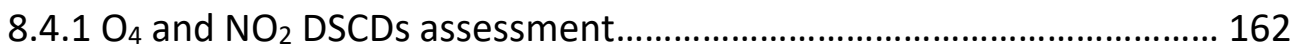

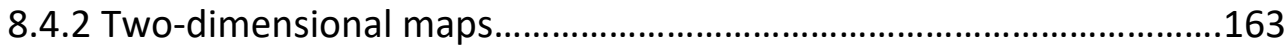

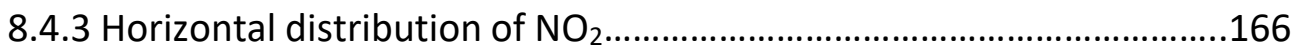

8.4.4 Correlation with the in-situ air quality monitoring stations of Madrid.....

9 CONCLUSIONS AND FUTURE WORK............................................173

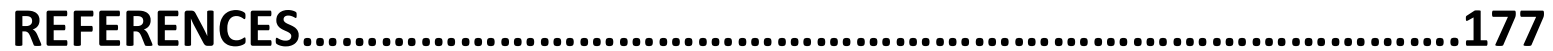





\section{List of figures}

Figure 2.1. Temperature and pressure vertical profiles, using the U.S. Standard Atmosphere profiles.

Figure 2.2. Flow of energy through the atmosphere of the Earth (diagram created by Kevin Trenberth, John Fasullo and Jeff Kiehl at the UCAR Center for Science Education).

Figure 2.3. Emitted spectral radiation by the Earth. The greenhouse gases keep part of this radiation within the atmosphere (figure taken from Honsberg and Bowden, 2019).

Figure 3.1. The Beer-Lambert law representation.

Figure 3.2. Absorption cross sections for $\mathrm{O}_{3}$.

Figure 3.3. Absorption cross sections for the different trace gases in the UV-VIS regions. This figure is taken from the book "Atmospheric Trace Gases" document version: 20.03.2014 (C.Kern).

Figure 3.4. Basic geometry of the MAXDOAS measurements. This figure comes from Nicole and Frank 2004.

Figure 3.5. Example of a DOAS analysis in Madrid on May 11, 2019 at around noon.

Figure 4.1. Basic diagram of a light scattering event.

Figure 4.2. Rayleigh function with respect to wavelength.

Figure 4.3. Rayleigh scattering coefficient at different wavelengths.

Figure 4.4. Mie scattering phase function for different values of the parameter $\mathrm{g}$.

Figure 4.5. U.S. Standard Atmosphere number density profile.

Figure 4.6. Geometry for a given SZA $\Theta$.

Figure 4.7. Scattering weights for different VEA.

Figure 5.1. MAXDOAS-1D location in Madrid (Spain).

Figure 5.2. MAXDOAS-1D on the roof of the ICA building.

Figure 5.3. MAXDOAS-1D measurements software. The interface includes the spectra collected by each optical fiber, along with all the parameters.

Figure 5.4. MAXDOAS-2D geometry diagram, the background of this picture represents the Madrid city center taken by (C) Google Maps. 
Figure 5.5. a) Aluminum tower with the instrument installed on top of it; b) MAXDOAS-2D instrument; c) MAXDOAS-2D scheme.

Figure 5.6. Solar scan for the alignment of the MAXDOAS-2D instrument.

Figure 5.7. Electronic offset and dark current corrections in the UV region for the MAXDOAS-1D instrument.

Figure 5.8. Example of the wavelength calibration of a spectrum. It was a zenith spectrum collected with the MAXDOAS-2D in Madrid at around noon on May 11, 2019. The estimated spectral resolution (FWHM) is of $0.54 \mathrm{~nm}$, with an estimated wavelength shift correction of $0.03 \mathrm{~nm}$.

Figure 6.1. Examples of $\mathrm{O}_{4}$ and HONO spectral fits in the UV region on April 6, 2016.

Figure 6.2. Example of a HONO spectral fit in the UV region on April 6, 2016.

Figure 6.3. Comparison between simulated and measured $\mathrm{O}_{4}$ DSCDs on January 16, 2016 (clear sky day).

Figure 6.4. Comparison and correlation between measured and simulated $\mathrm{O}_{4} \mathrm{DSCD}$ on January 16, 2016.

Figure 6.5. Vertical profiles of aerosol extinction coefficients for the UV region in Madrid on January 16, 2016.

Figure 6.6. Comparison and correlation between measured and simulated $\mathrm{NO}_{2}$ DSCDs on January 16, 2016.

Figure 6.7. Vertical profiles of $\mathrm{NO}_{2}$ for the UV region in Madrid on January 16, 2016.

Figure 6.8. Comparison and correlation between measured and simulated HONO DSCDs on January 16, 2016.

Figure 6.9. HONO vertical concentration profiles in Madrid on January 16, 2016.

Figure 6.10. Annual correlation of measured and simulated DSCDs of $\mathrm{O}_{4}$ and $\mathrm{NO}_{2}$.

Figure 6.11. Annual correlation of measured and simulated DSCDs of HONO.

Figure 6.12. Seasonal, diurnal variation of HONO surface mixing ratio and VCDs.

Figure 6.13. Seasonal, diurnal variation of temperature and relative humidity in Madrid during 2016.

Figure 6.14. Seasonal diurnal profiles of HONO at 8 UTC. 
Figure 6.15. HONO monthly variation of relative concentration and VCD at 8 UTC.

Figure 6.16. Seasonal diurnal variation of $\mathrm{NO}_{2}$ relative concentration (of the first layer).

Figure 6.17. Seasonal and diurnal $\mathrm{OH}$ production rate from HONO photolysis.

Figure 7.1. Formaldehyde and glyoxal spectral fitting examples. The red curves are the calculated optical densities for each trace gas (using QDOAS) while the black curves represent the measured optical density and the residual of the analysis, and they stand for the goodness of the analysis. The spectrum was collected on April 6, 2016, at a VEA of $20^{\circ}$ and a SZA of $54^{\circ}$ for formaldehyde and at a VEA of $20^{\circ}$ and SZA of $65^{\circ}$ for glyoxal.

Figure 7.2. Long-term correlation of simulated and measured HCHO DSCDs throughout the year.

Figure 7.3. a) Measured and simulated HCHO DSCDs comparison, b) correlation between measured and simulated HCHO DSCDs, c) diurnal vertical profiles of $\mathrm{HCHO}$ mixing ratio d) HCHO surface mixing ratio. The measurements of these plots were all taken on April 6, 2016. Figure 7.4. Seasonally averaged diurnal surface mixing ratios and VCDs of HCHO.

Figure 7.5. Monthly variation of hourly averaged surface mixing ratios (left column) and VCDs (right column) for HCHO at three different UTC hours.

Figure 7.6. a) Measured and simulated $\mathrm{CHOCHO}$ DSCDs comparison, b) correlation between measured and simulated $\mathrm{CHOCHO}$ DSCDs, c) diurnal vertical profiles of $\mathrm{CHOCHO}$ mixing ratio d) $\mathrm{CHOCHO}$ surface mixing ratio. The measurements of these plots were all taken on November 10, 2016.

Figure 7.7. Glyoxal monthly variation at 09 UTC time of hourly averaged surface mixing ratios (a) and VCD (b).

Figure 7.8. Monthly variation of $\mathrm{R}_{\mathrm{GF}}$ calculated from hourly averaged $\mathrm{CHOCHO}$ and $\mathrm{HCHO}$ surface mixing ratios at 08 UTC time (blue curve) and monthly variation of $\mathrm{NO}_{2}$ surface mixing ratio at 08 UTC time (green curve).

Figure 7.9. Monthly variation of the ratio between biogenic and anthropogenic VOCs emissions in Madrid for the year 2015. The ratio was taken using hourly averaged values of the emissions inventories at 08 UTC. 
Figure 8.1. Spectral detection of $\mathrm{O}_{4}(\mathrm{a})$ and (c) and $\mathrm{NO}_{2}(\mathrm{~b})$ and (d), red lines represent the calculated optical densities and black lines are the measured optical densities.

Figure 8.2. Comparison of retrieved aerosols using two different atmospheric profiles: the U.S. Standard Atmosphere (atmosphere A) and the U.S. Standard Atmosphere adapted to the altitude above sea level of Madrid (atmosphere B).

Figure 8.3. $\mathrm{NO}_{2}$ retrieval comparison using two different atmospheric profiles: the US Standard Atmosphere (atmosphere A) and the US Standard Atmosphere adapted to the altitude above sea level of Madrid (atmosphere B).

Figure 8.4. Comparison of the simulated $\mathrm{O}_{4}$ DSCDs and the retrieved extinction coefficients using two different a priori column-integrated extinctions: a total extinction of 0.05 (atmosphere C) and a total extinction of 0.1 (atmosphere D).

Figure 8.5. Comparison of the simulated $\mathrm{O}_{4}$ DSCDs and the retrieved extinction coefficients using two different surface albedo coefficients: 0.07 (atmosphere E) and 0.7 (atmosphere F).

Figure 8.6. Comparison between simulated and measured DSCDs of $\mathrm{O}_{4}$ and $\mathrm{NO}_{2}$.

Figure 8.7. Example of $\mathrm{O}_{4}$ and AOD retrievals in the UV region at 9 UTC on May 11, 2019. These contour plots are smoothed from adjacent VAA data points separated by $20^{\circ}$ in order to estimate the azimuthal distribution of the irradiance extinction coefficients over Madrid. Figure 8.8. $\mathrm{NO}_{2}$ vertical distribution retrieved in the UV region at 6 UTC and at 12 UTC on May 11, 2019. These contour plots are smoothed from adjacent VAA data points separated by $20^{\circ}$ in order to estimate the azimuthal distribution of $\mathrm{NO}_{2}$ over Madrid.

Figure 8.9. HONO vertical distribution retrieved at 6 UTC.

Figure 8.10. Polar plots of $\mathrm{NO}_{2}$ within the surface layer (0-200 m height) for May 11, 2019. Please note that these polar plots extend over a direction perpendicular to those shown in Figure 8.8. Here, circles are used for the UV (shorter horizontal light path) and triangles for the VIS (larger horizontal light path).

Figure 8.11. Polar plots of $\mathrm{NO}_{2}$ within the surface layer (0-200 $\mathrm{m}$ height) for May 11, 2019. Please note that these contours extend over a direction perpendicular to those shown in 
Figure 8.8. These contour plots are smoothed from adjacent VAA data points separated by $20^{\circ}$ and from the two different horizontal light paths in the radial direction.

Figure 8.12. Monitoring stations in Madrid, the picture was taken from @ Annual Plan 2019 Madrid.

Figure 8.13. Correlation between in-situ observations from Madrid's air quality monitoring network and those derived from the MAXDOAS-2D instrument for the surface layer (0-200 $\mathrm{m}$ height). 



\section{List of tables}

Table 2.1. Composition of the atmosphere of the Earth at sea level.

Table 6.1. DOAS fit settings for the retrieval of $\mathrm{O}_{4}$ and $\mathrm{NO}_{2}$ in the $\mathrm{UV}$ range.

Table 6.2. DOAS fit settings for the retrieval of HONO in the UV range.

Table 7.1. DOAS fit settings for the retrieval of glyoxal in the VIS range.

Table 7.2. DOAS fit settings for the retrieval of $\mathrm{HCHO}$ in the UV range.

Table 8.1. DOAS fit settings for the retrieval of $\mathrm{O}_{4}$ and $\mathrm{NO}_{2}(\mathrm{UV})$.

Table 8.2. DOAS fit settings for the retrieval of $\mathrm{O}_{4}$ and $\mathrm{NO}_{2}$ (VIS).

Table 8.3. Summary of average uncertainties of the retrieval in both spectral regions. 



\section{List of abbreviations}

\begin{tabular}{|c|c|}
\hline AERONET & AErosol RObotic NETwork \\
\hline AMF & Air Mass Factor \\
\hline AOD & Aerosol Optical Depth \\
\hline CCD & Charge-Coupled Device \\
\hline $\mathrm{CCN}$ & Cloud Condensation Nuclei \\
\hline CSIC & Consejo Superior de Investigaciones Científicas \\
\hline DOAS & Differential Optical Absorption Spectroscopy \\
\hline DSCD & Differential Slant Column Density \\
\hline EF & Enhancement Factor \\
\hline FWHM & Full Width at Half Maximum \\
\hline ICA & Instituto de Ciencias Agrarias \\
\hline LIDAR & Light Detection and Ranging \\
\hline MAXDOAS & Multi-AXis Differential Optical Absorption Spectroscopy \\
\hline NIWA & National Institute of Water and Atmospheric Research \\
\hline OD & Optical Density \\
\hline $\mathrm{R}_{\mathrm{GF}}$ & Ratio between Glyoxal and Formaldehyde \\
\hline RMS & Residual Mean Square \\
\hline RTM & Radiative Transfer Model \\
\hline SAA & Solar Azimuth Angle \\
\hline SEA & Solar Elevation Angle \\
\hline SI & International System of Units \\
\hline SOA & Secondary Organic Aerosols \\
\hline SZA & Solar Zenith Angle \\
\hline UPS & Uninterruptible Power Supply \\
\hline VAA & Viewing Azimuth Angle \\
\hline VCD & Vertical Column Density \\
\hline
\end{tabular}


VEA

WHO

WMO
Viewing Elevation Angle

World Health Organization

World Meteorological Organization 


\section{Abstract}

This thesis is focused on measurements of trace gases in the atmosphere of Madrid (Spain) and their assessment and relationship with urban pollution and air quality. The remote-sensing, ground-based Multi AXis Differential Optical Absorption Spectroscopy (MAXDOAS) technique has been used.

A complete analysis of $\mathrm{NO}_{2}, \mathrm{HONO}$, glyoxal and formaldehyde vertical concentration profiles in the urban atmosphere of Madrid has been performed. Our MAXDOAS-1D instrument (located within the city centre) was operating during the entire year of 2016 allowing the retrieval of diurnal and seasonal cycles of these trace gases. Using DOAS spectral fitting methods and a radiative transfer model, information on vertical columns and their altitude-distributed concentrations were retrieved. The RTM simulates the atmosphere, resulting in vertical profiles of the aerosols extinction coefficients, nitrogen dioxide, nitrous acid, glyoxal and formaldehyde concentrations.

A new MAXDOAS-2D instrument was built from scratch and a complete analysis of $\mathrm{O}_{4}$ and $\mathrm{NO}_{2}$ vertical concentration profiles in the urban atmosphere of Madrid (Spain) has been performed over two months (from May 6 to July 5, 2019). It provides a twodimensional view (in height and Viewing Azimuth Angle) of irradiance extinction and $\mathrm{NO}_{2}$ concentration profiles. Moreover, we also carried out the HONO analysis, providing vertical, two-dimensional profiles of this trace gas as well. We inferred data on the horizontal gradient of $\mathrm{NO}_{2}$ within the surface layer making use of the different light paths travelled by photons at different spectral regions. The "onion-peeling" method yielded peak values of $\mathrm{NO}_{2}$ in the early morning and in the southern section of the city (around $\left.180^{\circ} \mathrm{VAA}\right)$. We suggest that the new mesoscale information provided by the MAXDOAS-2D instrument may help in the study of pollution transport dynamics in Madrid. In addition, it is shown that this technique complements very well the in situ monitoring of trace gases, providing information at a larger spatial scale for the overall analysis of air pollution in Madrid. 



\section{Resumen}

Esta tesis está centrada en las medidas de gases traza en la atmósfera de Madrid (España) y su evaluación, así como la relación que tienen con la contaminación urbana y la calidad del aire en la ciudad. Para ello, la técnica de medidas desde tierra Multi AXis Differential Optical Absorption Spectroscopy (MAXDOAS) ha sido utilizada.

Se ha llevado a cabo un análisis completo de los perfiles verticales de concentración de $\mathrm{NO}_{2}, \mathrm{HONO}$, glioxal y formaldehído en la atmósfera de Madrid. Nuestro instrumento MAXDOAS-1D (situado en el centro de la ciudad) proporcionó medidas durante todo el año 2016, a partir de las cuales se obtuvieron perfiles diurnos y estacionales de todos los gases traza mencionados. Empleando la técnica DOAS, así como modelos de transferencia radiativa, se han calculado perfiles verticales de varios compuestos, proporcionando así información acerca de la distribución en altura de los mismos. Los modelos de transferencia radiativa simulan la atmósfera, junto con todos sus procesos físicos y químicos asociados. El resultado que facilitan son los perfiles verticales optimizados de diversos gases traza.

Por otra parte, un nuevo instrumento MAXDOAS-2D con capacidad para medir en dos dimensiones angulares ha sido desarrollado desde cero para esta tesis. De manera análoga a cómo se ha utilizado el instrumento MAXDOAS-1D, se han llevado a cabo análisis de perfiles verticales de $\mathrm{O}_{4}$ y $\mathrm{NO}_{2}$ en la atmósfera de Madrid durante dos meses del año 2019. Estos análisis proporcionan una visión bi-dimensional (en altura y en ángulo azimutal) de los perfiles verticales de extinción de irradiancia y de $\mathrm{NO}_{2}$. Además, también se ha llevado a cabo una evaluación de perfiles verticales de HONO para ambos meses. Asímismo, a partir de los espectros registrados se ha inferido el gradiente horizontal de $\mathrm{NO}_{2}$ dentro de la capa límite (la más cercana a la superficie terrestre) aplicando el concepto de que fotones pertenecientes a distintas regiones espectrales recorrerán -estadísticamente- caminos ópticos de distinta longitud. Para ello, se ha seguido la aproximación numérica conocida como "capas de cebolla", quedando como resultado general una mayor concentración superficial de $\mathrm{NO}_{2}$ en la parte sur de la ciudad (en torno a 180 de azimut, y de acuerdo con 
estudios previos sobre la distribución esperada de los contaminantes en Madrid). Sugerimos con este trabajo que la información de mesoescala proporcionada por el instrumento MAXDOAS-2D puede ser de gran ayuda en el estudio de la dinámica de transporte de contaminantes en Madrid. Además, se muestra aquí que esta técnica complementa muy bien la red de monitores in situ de la Comunidad de Madrid, siendo capaz de ofrecer información a una escala espacial mayor que dichos instrumentos para una evaluación más completa de la contaminación urbana entendida como un todo. 


\section{INTRODUCTION}

\subsection{Motivation}

Global warming, climate change and air pollution have become a great concern in our society nowadays, since they represent a major risk to human health (WHO, 2019) and they may cause severe environmental problems (affecting vegetation, fauna and ecosystems). Although these effects show a direct impact mainly on urban areas, they represent a worldwide threat. In this context, the atmosphere plays a pivotal role on all of these environmental issues. The study of the atmosphere -along with its interactions with other Earth components like the hydrosphere (water) of the lithosphere (Earth surface)- is key to understand the Earth as a global system. This includes the knowledge of gas and aerosol emissions, composition and physical and transportation phenomena that take place within the atmosphere, and its variation over time and space (from microscale up to mesoscale) (EEA, 2019).

The state of air pollution is often expressed as air quality, which usually represents the concentration of gaseous pollutants as well as size and -or- number of particulate matter that may affect human health, ecosystems, national heritage and regional climate (Monks et al., 2009). Though air quality is usually considered as a measure of the anthropogenic perturbation of the natural atmospheric state, the interactions with biogenic and other natural emissions and their respective feedback have to be considered as well.

Contrary to what happens in global or regional chemistry studies, urban atmospheric chemistry is characterized by a high density of emission sources (often difficult to evaluate), very strong concentration gradients and large pollution levels. Residence 
times in urban environments are usually relatively short, hence affecting the range of chemical processes that may occur. The strong influence of microscale magnitudes (for instance winds or climate parameters) in this type of atmosphere makes the assessment of these magnitudes of great interest.

Pollution has a fundamental impact on urban air quality. And although there are some natural sources of pollutants, such as volcanoes, swamps, dust or forest fires, the anthropogenic emissions are by far the dominant sources of pollution. There are three removal processes of pollutants: chemical conversion to non-polluting constituents, dry deposition (whereby gases are absorbed by plants, water or soil) and wet deposition -or removal by precipitation- which is only effective for water soluble species. There are, however, a number of tropospheric species capable of oxidizing these pollutants so that they become soluble. Although these species can be present in trace amounts, they play a pivotal role in the chemistry of urban atmospheres. For instance, the hydroxyl radical $(\mathrm{OH})$ is the most important oxidant in the atmosphere. These oxidants determine the lifetime and the abundance of trace species, acting as atmospheric regulators. The reverse is also true: the abundance of trace species regulates the oxidizing capacity of the atmosphere, since an increase in the emission of a given pollutant reduces the abundance of its principal oxidant (Saiz-Lopez et al., 2017). The resulting positive feedback may even eventually lead to an increase of other pollutants. This underlines the importance of understanding the oxidizing capacity in the troposphere in the context of air pollution research.

Over the years, especially in big cities, there has been an effort to measure and improve the knowledge on the state of the atmosphere, mainly regarding pollutants and air quality. In this regard, many cities have installed in situ monitors to measure some of the most important air pollutants. However, these in situ instruments -as of today- do not routinely measure other important trace gases present in the atmosphere (for instance nitrous acid (HONO) or glyoxal ( $\mathrm{CHOCHO})$ ). Note also that in situ measurements are only representative of the immediate surrounding of the instruments and at a surface level. 
There is therefore a need for mesoscale analysis (both in horizontal and vertical) of urban air pollution that could complement the in situ measurements. Hence here lies the principal goal of this thesis: mesoscale analysis of important atmospheric gases in the city of Madrid, as well as an assessment of their relationship and implications with respect to urban air quality.

\subsection{Objectives}

As described above, the atmospheric composition and chemistry make an impact in the general state of the atmosphere. In Madrid, a decrease in the $\mathrm{NO}_{2}$ levels has been associated with an increase in tropospheric ozone $\left(\mathrm{O}_{3}\right)$, which in turn can change the oxidizing capacity of the atmosphere (Saiz-Lopez et al., 2017). Trace gases are particularly important, and they are key in the chemistry of the atmosphere in spite of not being present in significant amounts.

Some in situ instruments provide measurements that are only representative of their surroundings and at the surface level. The goal of this work is to determine the vertical concentration profiles of trace gases (and aerosols) at mesoscale within the city of Madrid. The Differential Optical Absorption Spectroscopy (DOAS) technique is used in the work of this thesis. It is a widely used technique for the detection of atmospheric trace gases in the atmosphere. It is based on the wavelength dependent absorption of light by atmospheric constituents. Various trace gases can be identified simultaneously by their spectral signatures which act as an individual 'fingerprint'.

Ground-based DOAS observations of scattered sunlight are very suitable both for long term measurements of atmospheric constituents, and for measurements in the field using small portable instruments. The latter can be used, for example, to study emissions from volcanic plumes or the emission of reactive trace gases from remote salt lakes. Since those instruments are easy to maintain, they are ideally suited for the operation in remote regions with restricted technical infrastructure. 
In particular, this thesis work focused on some trace gases (nitrogen dioxide $\left(\mathrm{NO}_{2}\right)$, HONO, formaldehyde ( $\mathrm{HCHO}$ ) and $\mathrm{CHOCHO}$ ), which are key in the study of atmospheric chemistry, despite their relatively low concentrations (typically below $1 \%$ of the air molecules). The Multi AXis Differential Optical Absorption Spectroscopy (MAXDOAS) technique was used to monitor these key trace gases in the urban atmosphere of Madrid. 


\section{ATMOSPHERE OF THE EARTH}

In this chapter the main concepts and ideas concerning the atmosphere of the Earth are explained, along with its layers and the main atmospheric components. A brief description of the most relevant physical phenomena is also included in this chapter.

\subsection{Definition}

An atmosphere (from Greek atmos, haze) can be defined as a gas cover surrounding a star or a planet due to its own gravitational force. The atmosphere of the Earth can be divided into different layers. The most common classification is based on the temperature vertical profile. According to this scheme, the atmosphere is divided into the troposphere, the stratosphere, the mesosphere, the thermosphere and the exosphere (see Figure 2.1, more details in Section 2.1.1).

The frontiers that separate two different layers are called 'pause', as in the tropopause, that divides the troposphere from the stratosphere. In the troposphere, the energy flow is dominated by incoming solar radiation heating up the surface (Figure 2.2). Sensible (thermal energy that can be measured with a thermometer) and latent (energy originating from evaporation on the ground or in the ocean) heat are then transported from the ground up into the troposphere, and thermal energy is converted into potential energy. The updraft of an air parcel from the ground can be approximated by adiabatic cooling, which explains the temperature decline with height of about 6-10 K per km. This transport of latent heat is the main driver for the tropospheric weather and largely explains its turbulent behaviour. On the other hand, the energy balance in the stratosphere is dominated by radiative heating due to absorption of incoming radiation by trace gases, for 
example $\mathrm{O}_{3}$. This leads to an increase of temperature with altitude and thus a stratification of the air masses, hence the term 'stratosphere'.

\subsubsection{Layers of the atmosphere}

In a general way, the atmosphere of the Earth can be divided into lower and upper regions. The lower atmosphere is generally considered to extend to the top of the stratosphere, up to an altitude of about $50 \mathrm{~km}$. Our atmosphere is characterized by variations of temperature and pressure with height. In fact, it is indeed the variation of the average temperature profile with altitude which serves as the foundation for separating the layers of the atmosphere. Briefly, the regions of the atmosphere are classified as follows:

- Troposphere: the lowest layer of the atmosphere, extending from the surface of the Earth up to the tropopause, which is at an altitude of $10-15 \mathrm{~km}$ depending on latitude and time of year. Its principal characteristics are the decrease of temperature with height and a rapid vertical mixing of gases.

- Stratosphere: ranges from the tropopause to the stratopause (which is located at roughly 45 to $55 \mathrm{~km}$ altitude); temperature increases with altitude, leading to a layer in which vertical mixing is slow.

- Mesosphere: extends from the stratopause to the mesopause (at a height of 80$90 \mathrm{~km}$ ). Its temperature decreases with altitude up to the mesopause, which is the coldest point in the atmosphere. As the troposphere, the mesosphere is characterized by rapid vertical mixing.

- Thermosphere: the region above the mesopause that is characterized by high temperatures as a result of the absorption of incoming solar radiation by molecular nitrogen $\left(\mathrm{N}_{2}\right)$ and molecular oxygen $\left(\mathrm{O}_{2}\right)$ (specially at shorter wavelengths) and 
rapid vertical mixing. The ionosphere is a region that is located between the upper mesosphere and lower thermosphere where ions are produced by photoionization.

- Exosphere: the outermost region of the atmosphere (altitude higher than $500 \mathrm{~km}$ ). Here, gas molecules with sufficient energy can escape from the Earth gravitational attraction.

However, since this work is related to urban environments, we have focused mainly in the troposphere. The troposphere -a term coined by the British Meteorologist Sir Napier Shaw-, from the Greek word tropi, meaning turn- is a region of ceaseless turbulence and mixing. As stated above, the height of the tropopause changes with latitude and time: it is at a maximum height over the tropics, sloping downwards moving toward the poles; on average, it has a height of about $16 \mathrm{~km}$ in the equator, while it is located at about $8 \mathrm{~km}$ in the poles. Following the convention of the World Meteorological Organization (WMO), the tropopause is defined as the lowest level at which the rate of decrease in temperature with height is sustained at $\leq 2 \mathrm{~K} \mathrm{~km}^{-1}$ (Maddox and Mullendore, 2018). Although the troposphere accounts for only a small fraction of the atmospheric total height, it contains about $80 \%$ of its total mass, including almost all of the water vapor and trace gases. In the troposphere, the temperature decreases almost linearly with height. In dry air the rate of decrease with increasing altitude is of approximately $9 \mathrm{~K} \mathrm{~km}^{-1}$. The reason for this decline in temperature is the increasing distance from the Sun-warmed Earth. At the tropopause, the temperature has fallen to an average of $\sim 217 \mathrm{~K}$. The troposphere can also be divided into the planetary boundary layer, extending from the surface of the Earth up to roughly $1 \mathrm{~km}$ height, and the free troposphere (ranging from $1 \mathrm{~km}$ to the tropopause). The boundary layer is the most interesting atmospheric level for urban air quality research, although there can be relevant chemical cycles involving trace compounds within the free troposphere. Figure 2.1 shows the temperature and pressure vertical profiles using the U.S. Standard Atmosphere profiles. The three first atmospheric layers (troposphere, stratosphere and mesosphere) 
are depicted on the temperature profile, being clearly distinguished based on the changes in the temperature behavior with height.
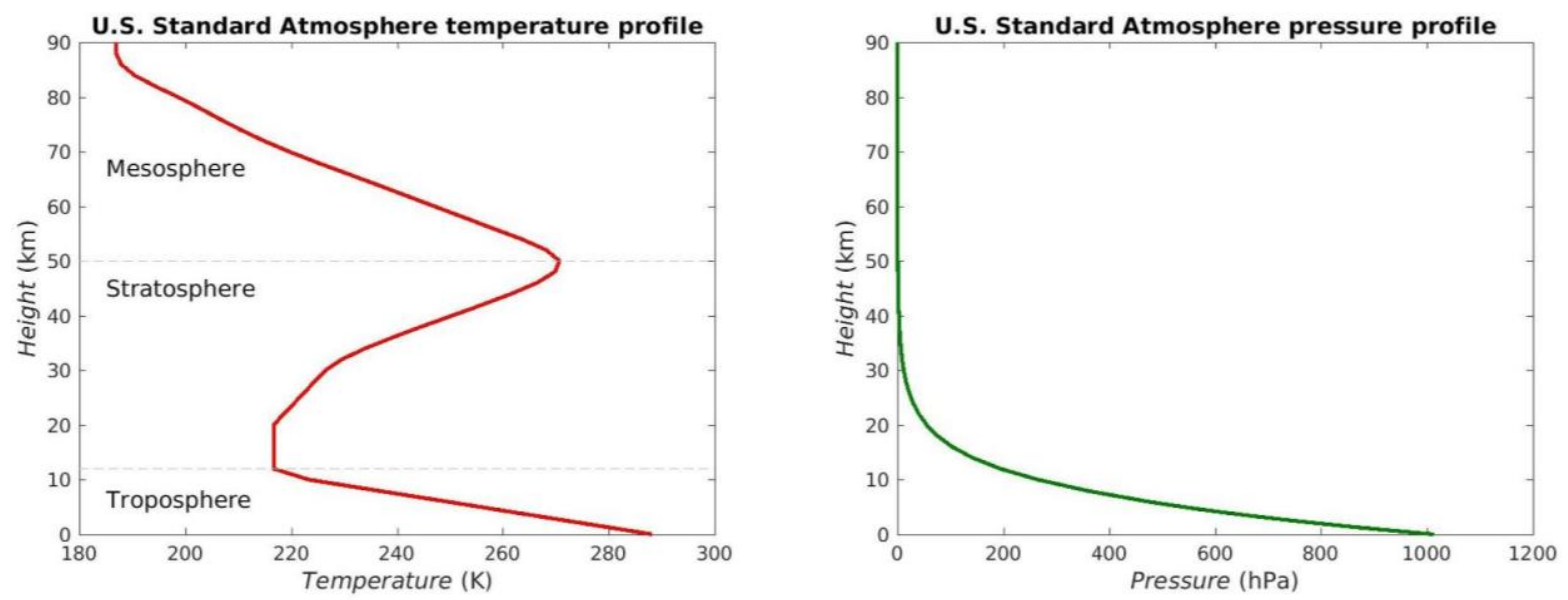

Figure 2.1. Temperature and pressure vertical profiles, using the U.S. Standard Atmosphere profiles.

\subsubsection{Global energy budget}

The energy budget of the Earth is crucial for the global climate. Weather and climate on Earth are defined by the distribution and sum of incoming radiation from the Sun. Outgoing longwave radiation balances the incoming absorbed solar radiation for an climatic equilibrium.

The diagram in Figure. 2.2 illustrates the flow of energy through the atmosphere of the Earth. It shows how energy contained in sunlight warms our planet, and how this energy becomes temporarily trapped as it flows away from the surface as longwave infrared radiation. This energy trap produces the greenhouse effect, the main driver of global warming (see Section 2.3 for more details).

Nevertheless, not all of the energy coming from the Sun reaches the atmosphere of the Earth, let alone the surface: one reason is that the clouds or the atmosphere itself 
absorb or reflect part of the radiation. Moreover, some of the energy which is reflected back to the atmosphere is absorbed by clouds or greenhouse gases present in the atmosphere. Then, it is re-emitted downwards.

\section{Global Energy Flows W $\mathrm{m}^{-2}$}

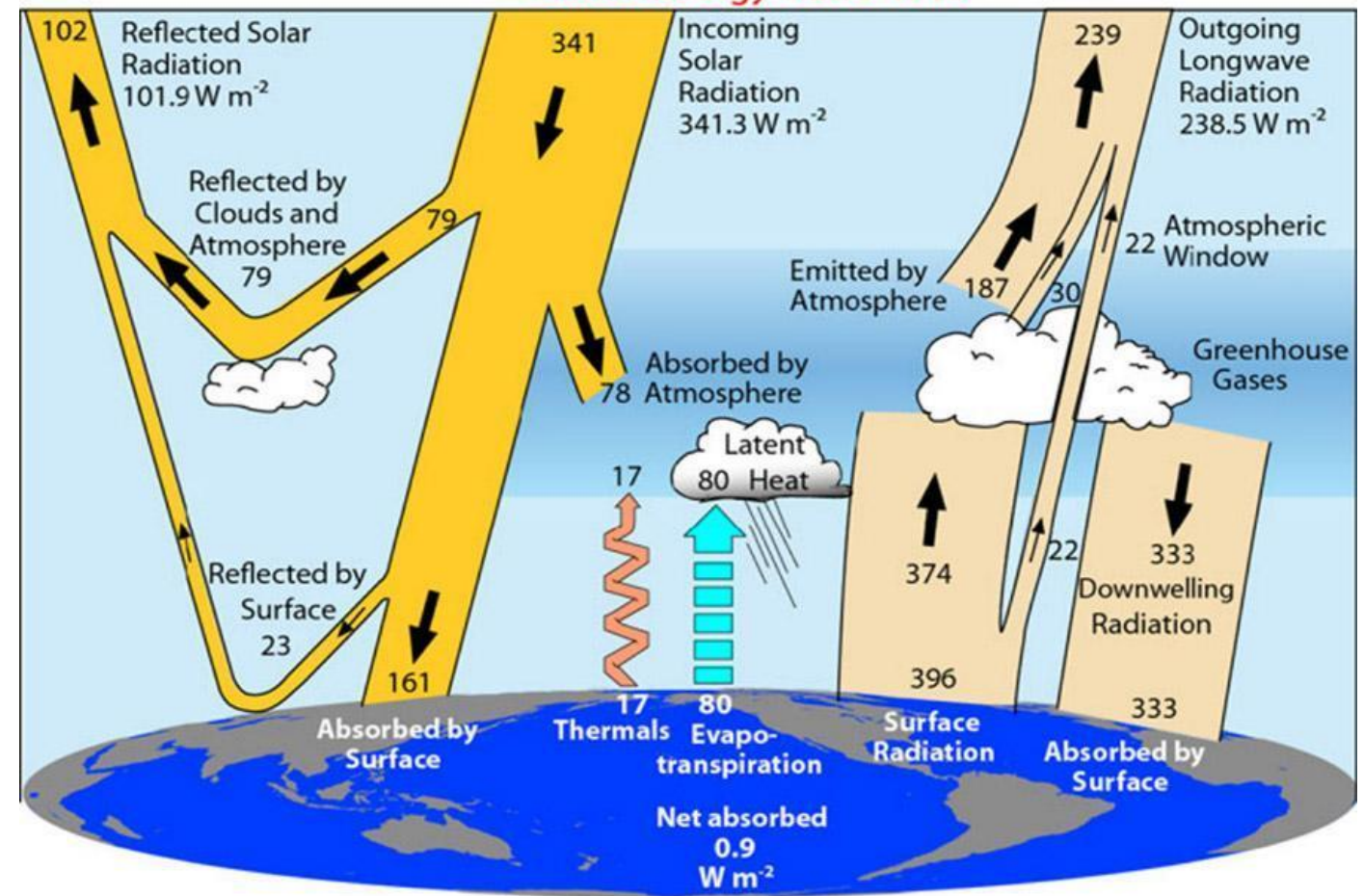

Figure 2.2. Flow of energy through the atmosphere of the Earth (diagram created by Kevin Trenberth, John Fasullo and Jeff Kiehl at the UCAR Center for Science Education).

Basically, all the energy that reaches the Earth comes from the Sun as we can see in Figure 2.2. The average temperature on Earth remains fairly constant, indicating that the Earth as a system (including the atmosphere) loses as much energy by re-radiating back into space as is received by radiation from the Sun. The accounting for the incoming and outgoing radiant energy constitutes the main components in the energy balance of the Earth. The atmosphere, although it may appear to be transparent to radiation, plays a very important role in the aforementioned energy balance. In fact, the atmosphere controls the amount of solar radiation that actually reaches the surface and, at the same time, restrains how much outgoing terrestrial radiation escapes into space. 
The energy budget can change due to internal variability and external forcing, which can be either natural (e.g. variations in solar intensity) or originated by humans. People in their activities emit a large quantity of gases to the atmosphere, most of them are dangerous to human health and others can be greenhouse gases (explained in section 2.3), the latter causing global warming. The exposure to air pollutants can be harmful and severely affect human health. For example, long exposure to these compounds can produce several health problems like allergies, irritation in the mucosa conducts, pulmonary diseases, cardiovascular illnesses, asthma and lung or other cancers.

\subsection{Composition of the atmosphere}

The main constituents of the atmosphere in the Earth are $\mathrm{O}_{2}, \mathrm{~N}_{2}$ and argon ( $\mathrm{Ar}$, see Table 2.1 for standard dry air composition). The atmosphere also contains water vapor not included among the dry air components cited above- which is present at an average volume fraction of around $1 \%$ at sea level (and roughly $0.4 \%$ over the entire atmosphere). However, water vapor strongly varies with location, reaching up to $5 \%$ in volume in hot, humid places. In the stratosphere, on the other hand, the mixing ratio of water vapor is fairly steady: about 3-4 ppmv (parts per million in volume, see Section 3.9 for more details concerning atmospheric measurements and units). All the remaining gases (typically in

volume fractions much lower than $1 \%$ ) are therefore called trace gases. Some examples of these so-called trace gases are: carbon dioxide $\left(\mathrm{CO}_{2}\right)$, methane $\left(\mathrm{CH}_{4}\right)$, nitrous oxides or $\mathrm{O}_{3}$. There are also some noble gases included. Despite their low concentration, these trace gases are key for the atmospheric chemistry processes.

Regarding the stable main constituents, the atmosphere is well mixed and the concentrations are basically proportional to the atmospheric pressure profile (Fig 2.1). In contrast to the main constituents, trace gas concentrations are significantly affected by complex production and destruction cycles and may therefore vary strongly within space and time. 
Table 2.1. Composition of the atmosphere of the Earth at sea level.

\begin{tabular}{|c|c|c|}
\hline Gas & Symbol & Volume Fraction (\%) \\
\hline Nitrogen & $\mathrm{N}_{2}$ & 78.09 \\
\hline Oxygen & $\mathrm{O}_{2}$ & 20.95 \\
\hline Argon & $\mathrm{Ar}$ & 0.93 \\
\hline Carbon Dioxide & $\mathrm{CO}_{2}$ & 0.04 \\
\hline Trace Gases & - & $<1$ \\
\hline
\end{tabular}

\subsubsection{Main atmospheric trace compounds}

Within the category of atmospheric trace gases, there are some of them which will be present very often in our DOAS analysis. The $\mathrm{O}_{4}$ molecule is the dimer of the oxygen molecule $\mathrm{O}_{2}$. It is formed when two oxygen molecules collide. Therefore, its concentration is proportional to the square of the oxygen concentration in the atmosphere. For this reason, the integrated vertical column of $\mathrm{O}_{4}$ is given in units of molecules $\mathrm{cm}^{-5}$. Its vertical profile is an exponentially decreasing (with respect to altitude) curve with a scale height of approximately $4 \mathrm{~km}$. Since the vertical profile of the $\mathrm{O}_{4}$ concentration is well known, its measured column densities can be used to gain insight into photon paths in the atmosphere, i.e. light path enhancement due to aerosols and clouds.

The nitrogen oxides -i.e. nitrogen monoxide (NO) and $\mathrm{NO}_{2}$ - are together referred to as $\mathrm{NO}_{\mathrm{x}}$. They are one of the most problematic pollutants in urban areas not only in developing, but also in industrialised countries. They are key species in the control of tropospheric ozone production and they play an important role in the formation of the socalled urban smog (for instance the famous 'Los Angeles Smog'). Moreover, the 
concentration of $\mathrm{NO}_{x}$ has a strong influence on the atmospheric level of $\mathrm{OH}$, which initiates the degradation of most oxidizable trace gases.

On the other hand, atmospheric aerosols are a key component of the atmosphere. In particular, they have a strong impact on the radiation budget. Anthropogenic changes in the aerosol loading and its composition may contribute significantly to climate change and even affect human health. However, the lack of detailed knowledge on the aerosols optical properties are one of the largest uncertainty sources in climate forcing analysis (Wagner et al., 2004).

The influence of aerosols is rather complex, since it depends on several variables like their composition, size distribution or shape. There are different ways of measuring the aerosol loading: in-situ instruments provide information about particles, but they are limited to the surroundings of the instrument; there are also some remote sensing methods, for instance Sun photometers and LIDAR (acronym for LIght Detection And Ranging), which offer very precise measurements but for the total aerosol extinction; also information on the scattering phase function can be derived. One of the main disadvantages of Sun photometers is that they can only measure optical properties of the total atmospheric column. In addition, since they observe the absolute value of the solar radiation, the requirements on instrument calibration and stability are high, this instrument is used by AERONET (AErosol RObotic NETwork) to measure the Aerosol Optical Depth (AOD).

In addition to the aforementioned trace gases, there are some others which are important for urban environments, for instance: $\mathrm{O}_{3}, \mathrm{HCHO}, \mathrm{HONO}$ or glyoxal.

\subsection{The greenhouse effect}

As mentioned in the previous section, the mean overall temperature of the Earth is rather steady, due to an equilibrium established between the incoming radiation from the 
Sun and the energy radiated into space by the Earth. The outgoing radiation emitted by the Earth is strongly affected by the presence and composition of the atmosphere. If we had no atmosphere, as on the Moon, the average temperature on the surface of the Earth would be about $-18{ }^{\circ} \mathrm{C}$. However, there are some gases that either absorb or reflect back to Earth some of the outgoing radiation, thereby keeping this energy in the atmosphere and warming the Earth as a whole. Therefore, the atmosphere causes the temperature of the Earth to increase from the $-18{ }^{\circ} \mathrm{C}$ that would have a non-atmosphere Earth up to the mean actual value of about $15^{\circ} \mathrm{C}\left(33^{\circ} \mathrm{C}\right.$ above the mean temperature at the surface of the Moon). This effect is the famous greenhouse effect, and it is important to remark that it is a natural effect, indeed a critical factor to supporting life as we know it. However, human activities (industries, use of fossil fuels, clearance of forests and so on) have accelerated the greenhouse effect, being key in global warming. A natural background level of $\mathrm{CO}_{2}$ of 270 ppmv in the atmosphere acts as the main natural greenhouse gas. $\mathrm{CO}_{2}$ absorbs strongly in the 13-19 $\mu \mathrm{m}$ wavelength band while water vapor, another atmospheric trace gas, absorbs strongly in the 4-7 $\mu \mathrm{m}$ wavelength band. Between the two cover a significant part of the spectral radiation emitted by the Earth, therefore most of this outgoing radiation (70\%) escapes in the window between 7-13 $\mu \mathrm{m}$.

Nonetheless, human activities are increasingly releasing anthropogenic gases into the atmosphere, $\mathrm{CO}_{2}$ for example. Some of them even partially absorb in the 7-13 $\mu \mathrm{m}$ wavelength window through which heat radiated by the surface would normally escape to space, particularly $\mathrm{CH}_{4}, \mathrm{O}_{3}$, nitrous oxides and chlorofluorocarbons (CFCs). These anthropogenic gases prevent the normal escape of energy and are leading to an increase in terrestrial temperature. Present evidence suggests that effective $\mathrm{CO}_{2}$ levels will double by 2030 , causing global warming in the order of $1-4{ }^{\circ} \mathrm{C}$. This would lead to changes in wind patterns and rainfall and as a result may cause the interior of continents to dry out and cause the oceans to rise. Further increases in the release of anthropogenic gases would, of course, cause more severe effects. Some time ago there was considerable discussion about whether the warming was a trend or a statistical fluctuation. There was some hope that 
the average temperatures would go down again into the level of the statistical average. There is still some debate about it, however, in the intervening years the mean temperature on Earth has continued to rise, hence supporting the claims of global warming, which is widely accepted in all of scientific fields.

Figure 2.3 shows the expected spectrum of energy emitted from the Earth (yellow region). The observed spectrum (measured above the atmosphere) indicates that a noticeable fraction of that energy is either absorbed or reflected back to the surface by various gases in the atmosphere.

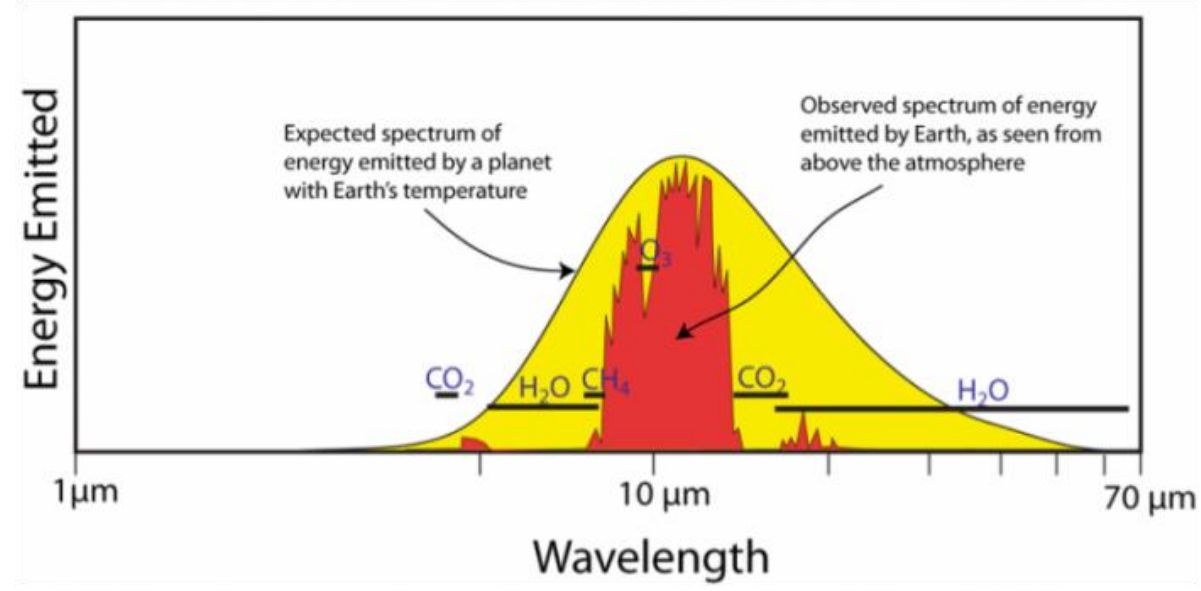

Figure 2.3. Emitted spectral radiation by the Earth. The greenhouse gases keep part of this radiation within the atmosphere (figure taken from Honsberg and Bowden, 2019).

Human activities have now reached a scale where they are impacting on the environment of the planet. The side-effects could be devastating and technologies with low environmental impact and no greenhouse gas emissions are likely to be of increasing importance over the coming decades. Since the energy sector is the major producer of greenhouse gases via the combustion of fossil fuels, technologies such as photovoltaics must increasingly be used. 


\subsection{Properties of light through the atmosphere}

Sunlight is a form of electromagnetic radiation and the visible light that we are able to see everyday as human beings is only a small fraction of the electromagnetic spectrum radiated by the Sun. The electromagnetic spectrum describes light as a wave which has a particular wavelength. The description of light as a wave first gained acceptance in the early 1800s when experiments performed by Thomas Young, François Aragon and Augustin Jean Fresnel showed interference effects in light beams, indicating that light is made of waves. By the late 1860s light was viewed as part of the electromagnetic spectrum. However, in the late 1800s a problem with the wave-based view of light became apparent when experiments measuring the spectrum of wavelengths from heated objects could not be explained using the wave-based equations of light. Planck proposed that the total energy of light is made up of indistinguishable energy elements, or a quanta of energy. Einstein, while examining the photoelectric effect (the release of electrons from certain metals and semiconductors when struck by light), correctly distinguished the values of these quantum energy elements. For their work in this area Planck and Einstein won the Nobel prize for physics in 1918 and 1921, respectively, and based on this work, light may be viewed as consisting of "packets" or particles of energy, called photons.

Today, quantum mechanics explain both the observations of the wave nature and the particle nature of light. In quantum mechanics, a photon, like all other quantummechanical particles such as electrons, protons, etc, is most accurately pictured as a "wavepacket". A wave packet is defined as a collection of waves which may interact in such a way that the wave-packet may either appear spatially localized (in a similar fashion as a square wave which results from the addition of an infinite number of sine waves), or may alternately appear simply as a wave. In the cases where the wave-packet is spatially localized, it acts as a particle. Therefore, depending on the situation, a photon may appear as either a wave or as a particle and this concept is called wave-particle duality. 
There are several key characteristics of the incident solar energy which are critical in determining how the incident sunlight interacts with any given object:

- The spectral content of the incident light.

- The radiant power density.

- The angle at which the incident solar radiation strikes at the surface of the object.

- The radiant energy from the Sun throughout a year or day.

A photon is characterized by either a wavelength, denoted by $\lambda$, or equivalently an energy, denoted by $E$. Radiation is emitted from matter when an electron drops to a lower level of energy. The difference in energy between the initial and final levels, $\Delta E$, is related to the frequency of the emitted radiation by Equation 2.1.

$$
\Delta E=h^{*} c / \lambda
$$

where $h$ is the Planck's constant ( $h=6.626 * 10^{-34} \mathrm{~J} \mathrm{~s}$ ), and $c$ is the speed of light in vacuum $\left(c=2.9979 * 10^{8} \mathrm{~m} \mathrm{~s}^{-1}\right)$. When the energy difference $\Delta E$ is large, the frequency of the excited photon is high (very small wavelength) and the radiation is in the X-ray or gamma-ray region. Equation 2.1 also applies to the absorption of a photon of a given energy by a molecule. Thus a molecule can absorb radiant energy only if the wavelength of the radiation corresponds to the difference between two of its energy levels. Since the spacing between energy levels is, in general, different for molecules of different composition and shape, the absorption of radiant energy by molecules of differing structure occurs in different regions of the electromagnetic spectrum. 


\subsubsection{Spectral irradiance}

There are many ways of mathematically treating light energy, but the most common way of expressing the energy flow of light in atmospheric sciences is the so-called spectral irradiance. It is the irradiance (energy flux, i.e. energy per time and transversal area) as a function of the photon wavelength (or frequency). In other words, it gives the power density received by a given surface at a particular wavelength. The units of the spectral irradiance using the International System of Units (SI) are $\mathrm{W} \mathrm{m}^{-3}$, however since light wavelength is usually expressed in $\mathrm{nm}$ within this field, we will find it more often in terms of $\mathrm{W} \mathrm{m}^{-2} \mathrm{~nm}^{-2}$. If needed, the photon flux can be obtained from the spectral irradiance by inserting the equivalence of the photon energy $E=h^{*} c / \lambda$. The total power density emitted from a light source can be calculated by integrating the spectral irradiance over all wavelengths or energies. The energy flux vector at several heights in the atmosphere will be key for developing the physical models that try to simulate the atmosphere (see Chapter 4).

\subsubsection{Solar radiation at the surface of the Earth}

While the incident solar radiation on the top of the atmosphere of the Earth is relatively constant, the radiation at the surface varies widely due to:

- atmospheric effects, including absorption and scattering.

- local variations in the atmosphere, such as water vapor, clouds and pollution.

- latitude of the location.

- the season of the year and the time of day.

The effects above have several impacts on the solar radiation received at the surface. These changes include variations in the power received, the spectral content of 
the light and the incident angle of light on a surface. In addition, a key change is that the variability of the solar radiation at a particular location increases dramatically. The variability is due to both local effects such as clouds and seasonal variations, as well as other effects such as the length of the day at a particular latitude.

\subsection{Atmospheric effects}

There are several atmospheric effects which exert an impact on the solar radiation that arrives at the surface of the Earth. The most important ones for atmospheric sciences are:

- a reduction in the power of the solar radiation due to absorption, scattering and reflection in the atmosphere.

- a change in the spectral content of the solar radiation due to greater absorption or scattering of some wavelengths.

- the introduction of a diffuse or indirect component into the solar radiation.

- local variations in the atmosphere (such as water vapor, clouds and pollution) which have additional effects on the incident power, spectrum and directionality.

\subsubsection{Absorption and scattering effects in the atmosphere}

As solar radiation passes through the atmosphere, gases, dust and aerosols absorb the incident photons. Specific gases, notably ozone, carbon dioxide and water vapor have very high absorption of photons that have energies close to the bond energies of these atmospheric gases. This absorption results in deep troughs in the spectral radiation curve of the Sun. 
While the absorption by specific gases in the atmosphere changes the spectral content of the terrestrial solar radiation, they have a relatively minor impact on the overall power. Instead, the major factor reducing the power from solar radiation is the absorption and scattering of light due to air molecules and dust. These absorption and scattering processes do not produce the mentioned deep troughs in the spectral irradiance, but rather cause a power reduction dependent on the photon path length through the atmosphere. When the Sun is overhead, the absorption due to these atmospheric elements causes a relatively uniform reduction across the visible spectrum, so the incident light appears white. However, for longer path lengths, higher energy (lower wavelength) light is more effectively absorbed and scattered. Hence in the morning and evening the Sun appears much redder and has a lower intensity than in the middle of the day.

One of the mechanisms for light scattering in the atmosphere is known as Rayleigh scattering which is caused by air molecules. Rayleigh scattering is particularly effective for short wavelength light (that is blue light) since it has a $\lambda^{-4}$ dependence. In addition to Rayleigh scattering, aerosols and dust particles contribute to the scattering of incident light known as Mie scattering.

Scattered light is undirected, and so it appears to be coming from any region of the sky. This light is called "diffuse" light. Since diffuse light is primarily "blue" light, the light that comes from regions of the sky other than where the Sun is, appears in blue. In the absence of scattering in the atmosphere, the sky would appear black, and the Sun would appear as a disk light source. On a clear day, about $10 \%$ of the total incident solar radiation is diffused (both absorption and scattering events in the atmosphere will be thoroughly detailed in Chapter 4). 


\section{FUNDAMENTALS OF DOAS}

Spectroscopy is the study of the interaction between radiation and matter as a function of the radiation wavelength or frequency. As we have seen in the previous chapter, within the atmosphere there can be processes of scattering, absorption or emission. The combination of all of them leads to the formation of absorption -or emissionlines (narrowband features) in the observed spectra. Absorption spectroscopy methods are well-established tools for the analysis of gases. It allows us to study their chemical composition and it has been key in improving our knowledge on the physical and chemical properties and processes in the atmosphere.

There are many spectroscopic techniques, and among them, DOAS is one of the most powerful and widely used techniques for the detection of atmospheric trace gases in the atmosphere. It is based on the wavelength dependent absorption of light by atmospheric constituents (Platt and Stutz, 2008). Various trace gases can be identified simultaneously by their spectral signatures.

The principle of the DOAS technique consists of separating the narrowband absorption features of molecular species from the broadband background that comes mainly from Rayleigh and Mie scattering as well as from instrumental effects (Plane and Saiz-Lopez, 2006). This leads to differential spectral structures that every trace gas possesses and that act like a "fingerprint", allowing us to differentiate between them.

The DOAS principle can be applied to a wide variety of light path configurations and observation platforms: from ground, on aircrafts, balloons and satellites, and by using 
either artificial light sources, scattered sunlight or direct sunlight (or moonlight) (Hönninger et al., 2004).

A major advantage of the DOAS technique is the ability to detect extremely weak absorptions with optical depths of around $10^{-4}$, which means it is sensitive enough to detect trace gases at typical atmospheric mixing ratios from as low as 0.1 parts per trillion (pptv) to several parts per billion (ppbv). As long as the wavelength region is carefully chosen, other trace gas species present in the sampled air mass do not influence the measurement. Another primary advantage of DOAS is the ability to measure absolute trace gas concentrations without any in situ calibration, once the absorption cross sections of the species are known. Additionally, DOAS measures the trace gases without chemical interference so it is particularly useful for the measurement of highly reactive species such as bromine monoxide ( $\mathrm{BrO}$ ) and iodine monoxide (IO). The simultaneous measurement of several trace gas species, by analysing the sum of their absorptions in one wavelength interval, provides information on the actual chemical composition of one air mass, typically in a few minutes. Finally, DOAS instruments are relatively simple, low-cost, portable and capable of real-time, unattended operation.

\subsection{The Beer-Lambert law}

When electromagnetic radiation passes through matter, its intensity is reduced by absorption and scattering. This process, called extinction, is described by the law of August Beer (Beer, 1854), which is illustrated in Figure 3.1. 


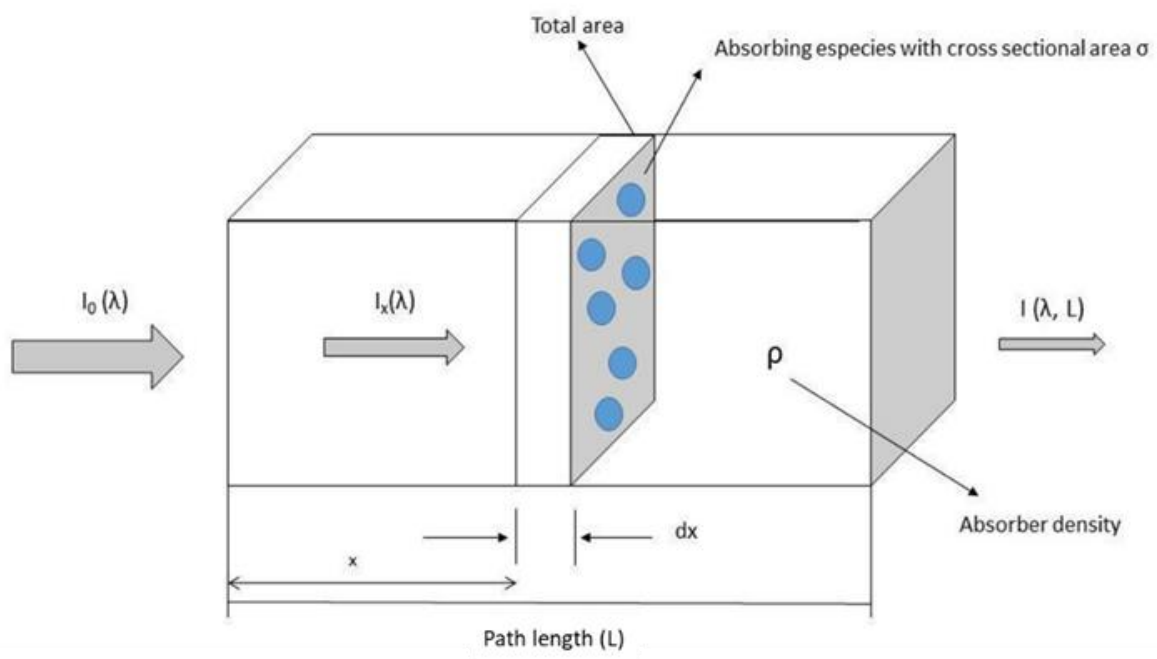

Figure 3.1. The Beer-Lambert law representation.

Let $I o(\lambda)$ be the intensity of a light beam at wavelength $\lambda$ before entering a certain volume where the absorber is present, and $I(\lambda)$ the intensity right after crossing that volume (measured at the same initial $\lambda$ ). Then both intensities are related by the BeerLambert law, which describes the exponential attenuation of light when traversing a volume due to its interaction with the matter that is located inside:

$$
I(\lambda, L)=I_{0}(\lambda) \exp \left(-\sum_{i} \int_{0}^{L} \sigma_{i}(\lambda) \rho_{i}(s) d s\right)
$$

where $\sigma_{i}(\lambda)$ is the absorption cross section of an absorber $i, \rho_{i}(s)$ represents its concentration along the light path, and as mentioned above $I_{0}(\lambda)$ and $I(\lambda)$ are respectively- the intensities before and after the considered absorption processes. In our case, $I_{0}(\lambda)$ is the Fraunhofer reference solar spectrum (with no atmospheric absorption) while $I(\lambda)$ stands for the recorded, ground-based spectrum, with all the absorption and scattering events included. The integral is done including the actual photon path and the results are added for every absorber.

To determine the concentration of a particular trace gas in the atmosphere, it would, in principle, be necessary to quantify all factors influencing the spectral intensity 
$I(\lambda)$ of a spectrum of light. In the laboratory, this can be achieved by removing the absorber from the light path. In the atmosphere, however, where this is impossible, the multiple factors influencing the intensity pose a dilemma. DOAS overcomes this challenge by using the fact that many processes in radiative transfer show broad or even smooth spectral characteristics, while certain trace gases exhibit narrow band absorption structures. The basic principle of DOAS is thus the separation of spectral structures in narrowband (varying strongly with wavelength) and broadband components (varying slowly with wavelength) in differential spectra, so that changes in narrowband absorptions can be detected. The use of Equation 3.1 in DOAS measurements requires its modification according to properties of radiative transfer in the atmosphere and the measurement principles of DOAS instruments. When performing the DOAS spectral fit, a magnitude called optical density $(\tau(\lambda))$ appears, as shown in Equation 3.2.

$$
\tau(\lambda)=\ln \left(\frac{I_{0}(\lambda)}{I(\lambda)}\right)
$$

thus we can expand the right side of Equation 3.2 once we remove the exponential after applying the natural logarithm in Equation 3.1.

$$
\tau(\lambda)=\sum_{i} \int_{0}^{L} \sigma_{i}(\lambda) \rho_{i}(s) d s
$$

The primary quantity measured by DOAS instruments is the Slant Column Density $(\mathrm{SCD})$, defined as the integrated concentration $\rho_{i}$ of a trace gas $i$ along a light path of length $L$ (Equation 3.4).

$$
S C D=\int_{0}^{L} \rho_{i}(s) d s
$$

Introducing this quantity into Equation 3.2, we get the basic DOAS fit equation:

$$
\tau(\lambda)=\sum_{i} S C D_{i} \times \sigma_{i}(\lambda)+\text { low order polynomial }
$$


By doing this, optical density vectors are obtained from the measured spectra, and a non-linear fit is carried out in order to get the optimal SCDs values. Each trace gas has its individual absorption cross section; therefore several absorbers can be measured simultaneously even if their absorption features are superimposed.

\subsection{Absorption cross sections}

The UltraViolet-VISible (UV-VIS) spectra of molecules (for this work we will consider the UV-VIS range as the spectral window of $300-500 \mathrm{~nm}$ ) exhibit vibrational and rotational structures. Frequently, the rotational structure consists of many lines, the spacing of which is smaller than their line-width (or which cannot be separated by the spectrometer), thus leading to a quasi-continuous absorption within a vibrational "band". The absorption cross section is a characteristic property of every species, and it depends on wavelength. It can be measured in the laboratory.

The ability to separate broad and narrow absorption features in the spectra is the foundation of DOAS, but restricts it to species with distinct and narrow absorption structures, typically of less than about $10 \mathrm{~nm}$ in width. There are, nevertheless, many detectable species with unique narrow band absorption features in the UV-VIS wavelength region, including $\mathrm{O}_{3}, \mathrm{NO}_{2}, \mathrm{NO}, \mathrm{NH}_{3}$ (ammonium), $\mathrm{HONO}, \mathrm{HCHO}, \mathrm{CHOCHO}, \mathrm{BrO}, \mathrm{IO}$, molecular iodine $\left(\mathrm{I}_{2}\right), \mathrm{OH}$ and $\mathrm{O}_{4}$. In theory, all gases that have these narrow absorption bands in the UV, visible or near InfraRed (IR, spectral region above $700 \mathrm{~nm}$ ) can be measured. However, the concentrations of these compounds in the atmosphere, and the detection limits of the DOAS instruments as of now, restrict the number of trace gases that can actually be detected.

The separation of the narrow- and broadband structures of the absorption cross section of a given trace gas is usually done subtracting the broad features with a low order polynomial, although it can also be achieved by means of a high-pass filter: 


$$
\sigma(\lambda)=\sigma_{\text {broad }}(\lambda)+\sigma_{\text {diff }}(\lambda)=\text { low order polynomial }+\sigma_{\text {diff }}(\lambda)
$$

Figure 3.2 shows an example of how the differential absorption cross section of a trace gas is obtained. As an initial simplification, we assume that the differential absorption cross section is independent of temperature and pressure. For certain passive DOAS applications, this assumption is difficult to sustain and would, in principle, require a rigorous numerical solution. There is, however, an approximate solution, which splits $\sigma^{\prime}$ into a small number of different absorption cross sections for individual temperature/pressure combinations (note that there is a correlation between pressure and temperature in the atmosphere). Nonetheless, it is a safe assumption in this work.
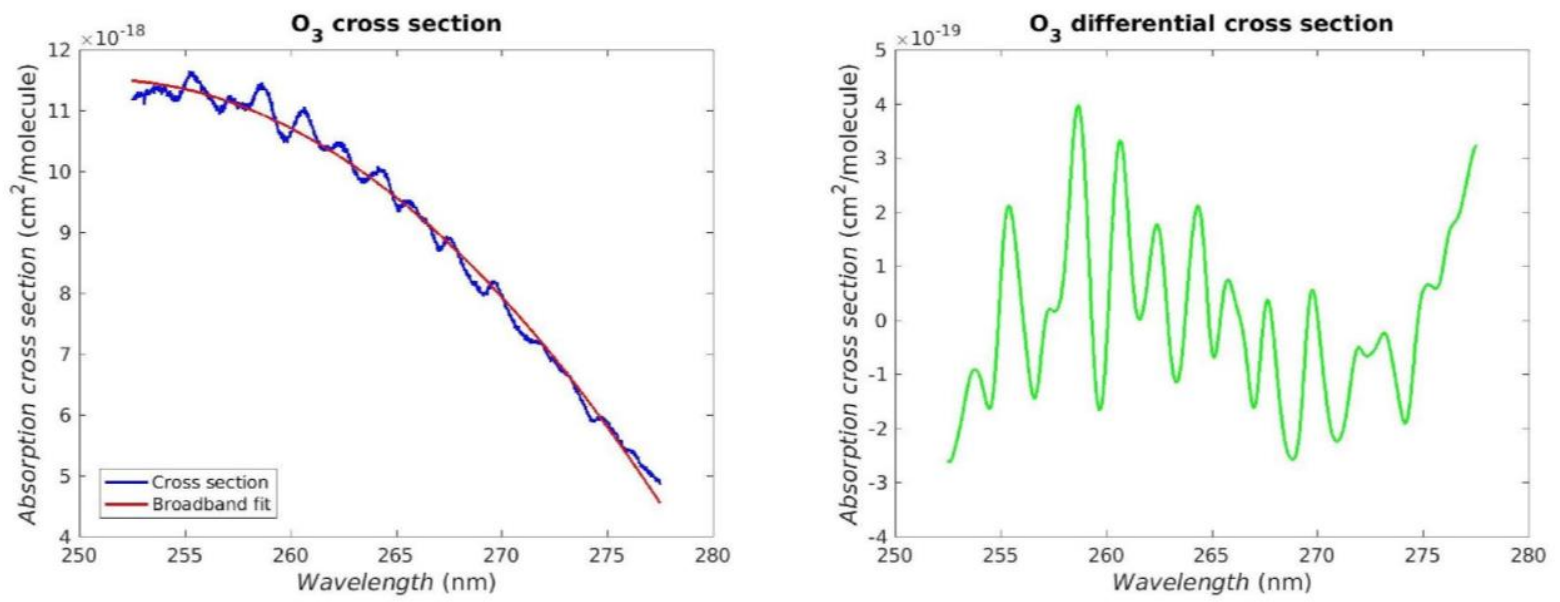

Figure 3.2. Absorption cross sections for $\mathrm{O}_{3}$.

Figure 3.3 shows the absorption cross sections of a number of trace gases that are regularly measured by DOAS. A number of features about these cross sections should be pointed out here. First and most importantly, each trace gas spectrum has a unique shape. Most of the trace gases only absorb in certain wavelength intervals. However, many spectral regions can contain a large number of simultaneous absorbers. For example, between 300 and $400 \mathrm{~nm}$, the following trace gases will show noticeable absorption features if they are present at high enough concentrations: $\mathrm{O}_{3}, \mathrm{SO}_{2}, \mathrm{NO}_{2}, \mathrm{HONO}, \mathrm{HCHO}$ and 
BrO. Because of their unique spectral structure, a separation of the absorptions is possible. From Equation 3.5, it is clear that spectral regions with higher $\sigma^{\prime}$ will show the largest optical densities. These spectral intervals are thus preferred for DOAS measurements since the sensitivity improves in these wavelength regions. In principle, each trace gas has an optimal wavelength interval. In practice, however, one has to often compromise in the choice of the wavelength interval to measure more than one trace gas simultaneously. Because expanding the wavelength window reduces the spectral resolution of typical grating spectrometers, the sensitivity is also reduced.

A condition for the successful differentiation of the trace gases is, however, that the absorption cross section of a certain gas does not accidentally contain absorption structures of other trace gases due to, say, impurities in the numerical analysis procedure. 


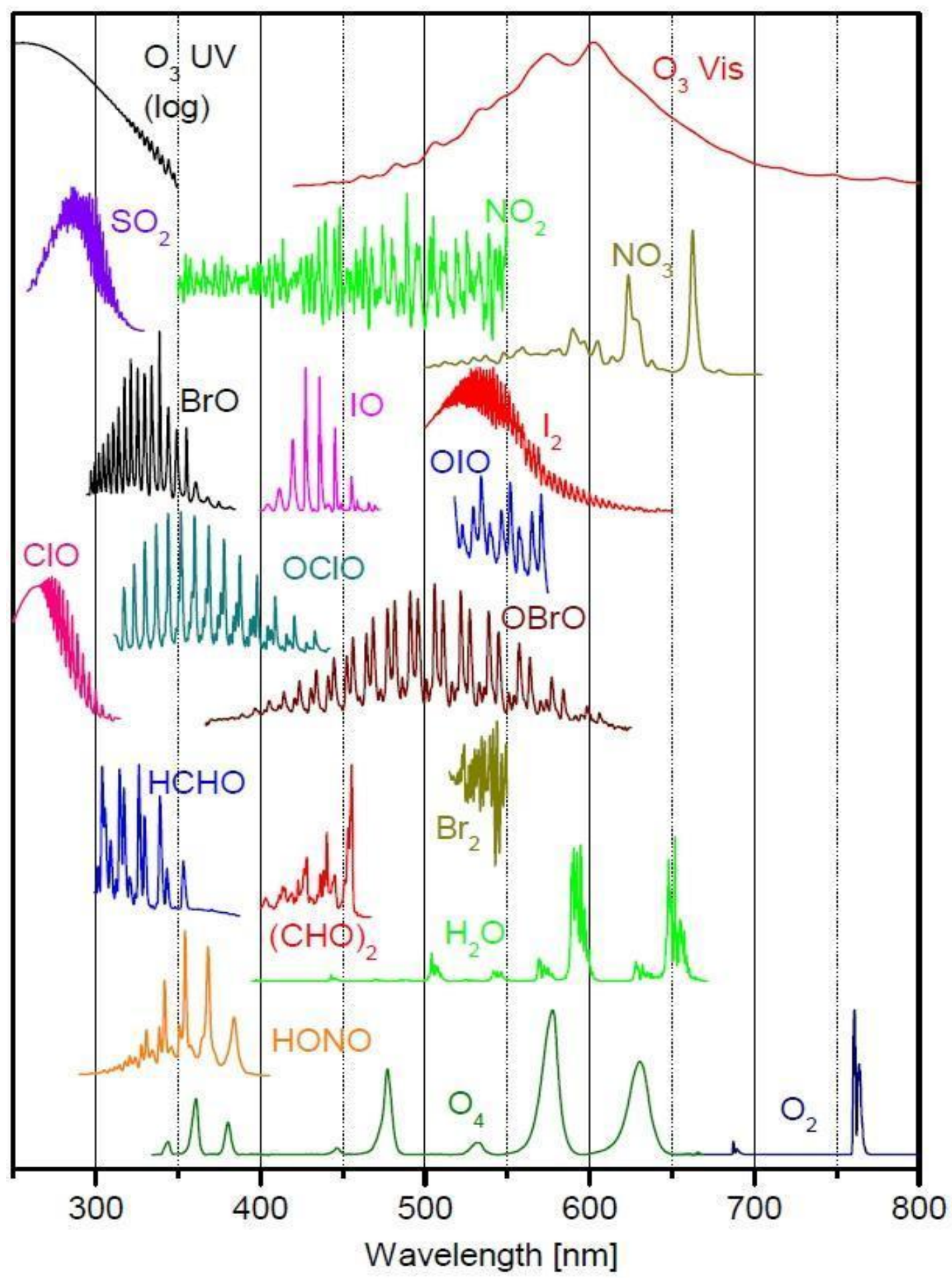

Figure 3.3. Absorption cross sections for the different trace gases in the UV-VIS regions. This figure is taken from the book "Atmospheric Trace Gases" document version: 20.03.2014 (C.Kern). 


\subsection{DOAS classification}

With respect to their light sources, there can be two types of DOAS systems. Active DOAS techniques, such as Long Path DOAS (LPDOAS), use an artificial light source often combined with retro-reflectors (Platt, 1994). LPDOAS has been widely used, especially for pollution studies (Saiz-Lopez et al., 2006). The advantage of this technique is that the light path is precisely known and, with long light paths, active DOAS is highly accurate and sensitive for measurements of trace gases near the ground. In addition, measurements can also be made at night and at wavelengths below $300 \mathrm{~nm}$, but active DOAS instruments require a much more complicated optical setup, more power, and higher maintenance than passive instruments (Plane and Saiz-Lopez, 2006). Passive DOAS uses light from the Sun, Moon or stars, so the instrumental setup is simpler than active DOAS, but since the light traverses an ensemble of paths through the whole atmosphere, the direct calculation of concentrations from absorptions is not possible. Thus radiative transfer calculations or geometric approximations are required in order to retrieve Vertical Column Densities (VCDs) or vertical concentrations profiles from the measurements. The range of passive DOAS geometries includes ground-based and balloon-borne direct sunlight DOAS, Zenith Scattered Light DOAS (ZSLDOAS), MultiAXis DOAS (MAXDOAS), Airborne MultiAXis DOAS (AMAXDOAS), Imaging DOAS (IDOAS), and satellite-borne occultation, nadir and limb DOAS (Platt and Stutz, 2008).

Ground-based DOAS observations of scattered sunlight are suitable both for long term measurements of atmospheric constituents, and for measurements in the field using small portable instruments. The latter can be used, for example, to study emissions from volcanic plumes or the emission of reactive trace gases from remote salt lakes. Since those instruments are easy to maintain, they are ideally suited for the operation in remote regions with restricted technical infrastructure. 


\subsection{Multi AXis Differential Optical Absorption Spectroscopy}

DOAS has been used extensively since the 1970 s and is a valuable tool for the measurement of several trace gas species, including $\mathrm{O}_{3}, \mathrm{NO}_{2}, \mathrm{NO}_{3}, \mathrm{BrO}$ and $\mathrm{O}_{4}$ (Hönninger, 2004). The first "off-axis" (viewing in directions other than the zenith) measurements were made by Sanders et al. (1993) using an off-axis zenith angle of $80^{\circ}$ in the direction of the Sun in order to observe stratospheric OClO for longer periods into the "polar night". As the sky is brighter in the direction of the Sun compared to the zenith at high elevation angles, this viewing direction improved the signal-to-noise ratio of the measurements. They also concluded that this approach enhanced absorption by tropospheric species, while absorptions for stratospheric species are comparable to the zenith observations (Solomon et al., 1987). Arpaq et al., 1994 used twilight off-axis observations to measure stratospheric BrO for the first time at mid-latitudes and Miller et al., 1997 used observations made at offaxis angles of $87^{\circ}$ and $85^{\circ}$ to measure stratospheric $\mathrm{BrO}$ and $\mathrm{OClO}$ related to polar sunrise boundary layer Ozone Depletion Events (ODEs) in Greenland.

The use of multiple off-axis observation angles to obtain increased information about the vertical distribution of trace gases in the proximity of the instrument was recently developed, and became known as MAXDOAS (Hönninger, et al., 2004). Measurements made with a telescope aimed at multiple elevation angles close to the horizon observe scattered sunlight that has traversed different paths through the atmosphere, with lower elevation angles typically observing light from longer absorption paths through the lowest layers relative to higher elevation angles (Figure 3.4). Thus, the slant column densities measured at low telescope elevation angles are enhanced for trace gases close to the instrument. In particular, this technique is highly sensitive to trace gas abundance in the lowest 1-2 km of the atmosphere for ground-based instruments, which is the typical upper range of the marine boundary layer height and the ideal range for pollution studies.

For illustration of the MAXDOAS measurement geometry, consider the geometric approximation shown in Figure 3.4. 


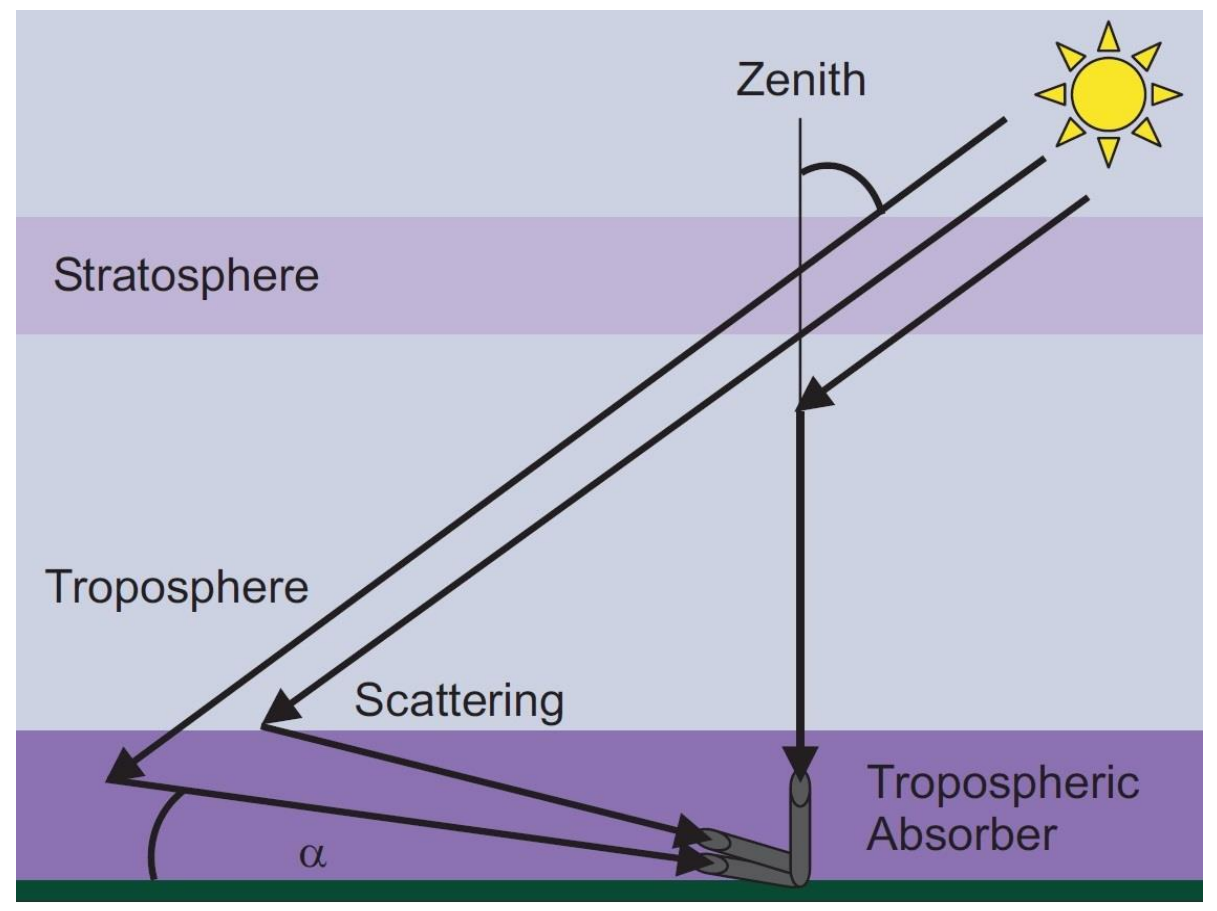

Figure 3.4. Basic geometry of the MAXDOAS measurements. This figure comes from Nicole and Frank, 2004.

For MAXDOAS applications, $I 0(\lambda)$ from Equation 3.1 stands for the solar spectrum (known as the Fraunhofer spectrum, with no Earth atmospheric absorptions), while $I(\lambda)$ represents the recorded ground-based spectrum, which includes all the absorption and scattering processes. However, and since the actual photon path is difficult to determine with accuracy (see Chapter 4), and determining the spectral instrumental effects with great accuracy is also very complex, the MAXDOAS calculations are done using relative absorptions between two different optical paths: a zenith spectrum -that contains less absorptions and is assumed as a reference spectrum- and other spectrum pointing to a given -usually low- elevation angle. This way, most of the instrumental effects, as well as the impact of the stratospheric events, will be canceled out. Therefore, the direct product of the method is the Differential Slant Column Density (DSCD), which can be defined as the difference in the integrated concentration of a given absorber between the two selected pointing directions (more details about the numerical procedure that lies behind can be found in Hönninger et al., 2004, Plane and Saiz-Lopez, 2006; Brooks et al., 2006; and Platt 
and Stutz, 2008). Finally, these DSCDs are used as the main input for the profile retrieval algorithms. Sometimes, it is useful to apply some geometric approximations in order to get a sense on the order of an estimated VCD (Hönninger et al., 2004):

$$
V C D \cong D S C D /\left[\frac{a}{\sin (\alpha)}+\frac{1-a}{\cos (\beta)}\right]
$$

where $a$ is the fraction of the total vertical trace gas column that resides below the typical scattering altitude. An absorber below the scattering altitude (e.g. in the boundary layer) enhances the light path by a factor of approximately $(1 / \sin (\alpha))$, where $\alpha$ is the elevation angle of the MAXDOAS instrument and $\beta$ represents the zenith angle of the Sun. We can observe that there is a strong effect of the instrumental elevation angles on the tropospheric light paths. Moreover, the higher the elevation angle of the instrument, the shorter the light path of the collected photons.

\subsection{DOAS technique retrieval}

There are two approaches for this process, depending on whether the fit is done directly over the spectra, generating a synthetic spectrum that simulates the measured one Basic Optical Absorption Spectroscopy (BOAS) or the spectral fit is carried out on the differential structures of the spectra (DOAS, which is the one we used in this study).

The DOAS analysis software used in this work is called QDOAS, and it was developed at BIRA-IASB, http://uv-vis.aeronomie.be/software/QDOAS/. DOAS employs non-linear least squares fitting to minimise the difference between the measured spectral radiances (in terms of optical density) and the differential absorption cross sections of the relevant absorbers that are present. In this technique, the wavelength window chosen is a key parameter, since it must be wide enough to contain distinctive narrowband absorptions features and avoid the interference of spectral features of other absorbers as much as possible. 
First, the reference spectrum (measured pointing at the zenith direction) is aligned -in terms of wavelength- with the Fraunhofer spectrum using a nonlinear least squares fitting (Aliwell et al., 2002). Then the other spectrum for the DOAS analysis is aligned with respect to the already aligned reference. After that, the Fraunhofer structures are eliminated by dividing both measured spectra. As a result of this ratio, some interpolation uncertainties might appear, thus sometimes a shift and/or stretch in the wavelength vector may be useful in order to improve the analysis.

Background spectral features that vary slowly with wavelength -for instance Rayleigh and Mie scattering- are handled as "pseudo-absorptions" and are approximated by low order polynomials to separate them from the fine spectral structures. The measured differential optical densities are fitted by a nonlinear least squares system to the differential absorption cross sections of each absorber (included in the analysis).

Moreover, the uncertainties in the estimated DSCDs retrieved by QDOAS are also provided as a one standard deviation of the parameter. Finally, also a residual vector of the analysis is rendered in optical density units. Figure 3.5 includes an example of a DOAS analysis using a couple of spectra to optimise the DSCDs: one measured a $1^{\circ}$ elevation angle and a zenith spectrum. 

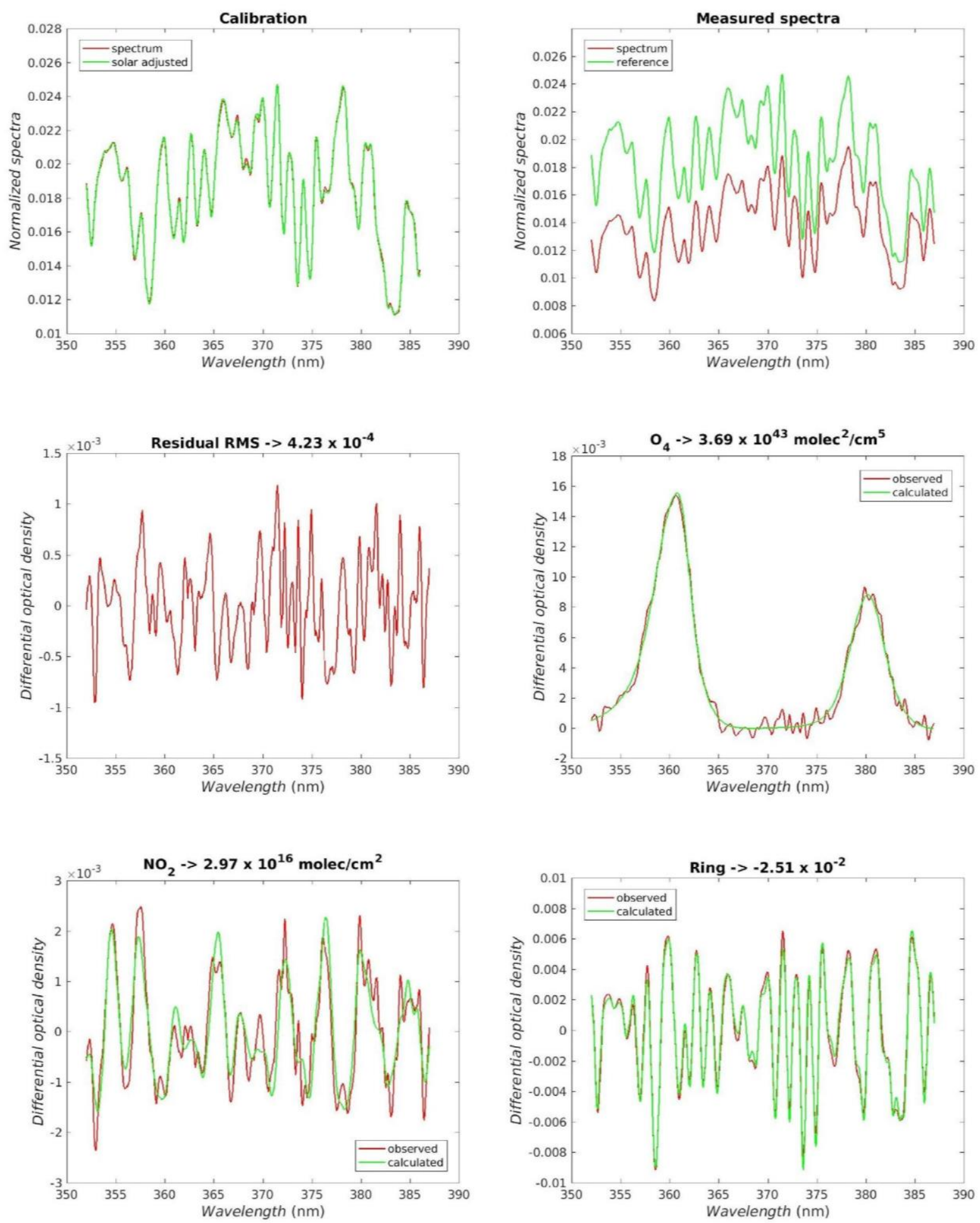

Figure 3.5. Example of a DOAS analysis in Madrid on May 11, 2019 at around noon. 


\subsection{Instrumental slit function}

The instrumental slit function or spectral resolution is defined as the Full Width at Half Maximum (FWHM) of the instrument function. The shape of a spectrum could change due to the entrance slit of the spectrometer grating and the focussing and dispersing optics when we use a spectrometer to measure the absorption spectrum. In addition to the mentioned effects, the nature of light itself has an impact on the uncertainty. All of these factors combine into this: what in theory should be a perfectly fine emission line (Dirac delta), clearly defined at a given wavelength, is indeed widened, often yielding a Gaussian shape. Therefore, the FWHM of a certain peak is considered as an estimation of the spectral resolution of a given instrument (in our case, a whole MAXDOAS instrument).

This parameter can be calculated using any type of spectrum. One common way consists of using a well calibrated emission lamp and evaluating the FWHM of the tabulated emission lines of the lamp. Another usual procedure in DOAS is to carry out the resolution estimation using the measured scattered sunlight spectra and comparing it to a highresolution solar atlas (as will be detailed in Section 5.6). However, a combination of the two methods was used for this work: a primary, coarse estimation of the resolution of our MAXDOAS instruments using emission lines in the laboratory, and subsequently adapt this resolution in the field within the context of a given measurements campaign.

The QDOAS software is able to estimate this parameter and it is needs that the instrumental resolution function remains stable and constant during a solar spectra measurement (Aliwell et al., 2002). When this function is obtained, the measured spectra (and/or the absorption cross sections of trace gases) can be corrected for instrumental effects by a convolution with the mentioned instrumental slit function. The numerical details of the procedure will be explained in Section 5.6. 


\subsection{The Ring effect}

In 1961, J.Grainger and J.Ring (Grainger and Ring, 1962) discovered that the depth of the Fraunhofer lines was smaller when examining scattered sunlight than when direct sunlight is observed. This was attributed to inelastic rotational Raman scattering, which slightly changes the wavelength of the scattered photon when the scattering occurs. This causes a "filling in" of strong absorption lines, as wavelengths on either side of the line are shifted into it.

The Ring effect is therefore the manifestation in atmospheric spectra that Rayleigh scattering is partially inelastic. As stated above, this inelastic component is composed of Raman scattering by air molecules, predominantly $\mathrm{N}_{2}$ and $\mathrm{O}_{2}$. This inelastic component varies from $3 \%$ to $4 \%$ of the total Rayleigh scattering over the prime atmospheric trace gas wavelength fitting region of $280-1000 \mathrm{~nm}$. But, although this fraction is small in its absolute value, it has indeed a noticeable effect in the spectral content of the optical density vector, specially given the relatively low optical density values which with we usually work with in the DOAS analysis. The Ring effect can be accounted for as an additional absorber in the DOAS fitting configuration. Thus, to first order, the correction for the Ring effect in atmospheric spectra is simply the convolution of the solar irradiance with the spectrum of Raman scattering by air molecules, where the cross sections for the dominant rotational Raman components are given by Chance and Spurr (Chance and Spurr, 1997).

\subsection{Observational geometries in DOAS}

\subsubsection{Solar Azimuth Angle}

The Solar Azimuth Angle (SAA) is the compass direction at which the Sun is located at a certain time. The origin of the SAA starts at the true north (SAA of 0 degrees) and goes clockwise. At solar noon, the Sun is always directly south in the northern hemisphere and directly north in the southern hemisphere. The azimuth angle varies throughout the day. 
At the equinoxes, the Sun rises directly east and sets directly west regardless of the latitude, thus making the azimuth angles $90^{\circ}$ at sunrise and $270^{\circ}$ at sunset.

\subsubsection{Solar Zenith Angle}

The Solar Zenith Angle (SZA) is the angle between the Sun and the zenith direction (gravitational vertical upwards). The SZA is related to the Solar Elevation Angle (SEA) since the last is the complementary angle of the former. This means that the SEA is the elevation angle of the Sun measured from the horizon.

$$
S Z A=90-S E A
$$

The SEA is $0^{\circ}$ at sunrise and $90^{\circ}$ when the Sun is directly overhead (which occurs for example at the equator on the spring and fall equinoxes).

\subsubsection{Viewing Azimuth Angle}

Using the same reference as the SAA, the Viewing Azimuth Angle (VAA) is the azimuthal angle -east wise with respect to the north- at which the line of sight of an instrument is pointing. The absolute difference between the SAA and the VAA is called the Relative Azimuth Angle (RAA).

\subsubsection{Viewing Elevation Angle}

The Viewing Elevation Angle (VEA, also Elevation Viewing Angle) is the angular height of the instrument line of sight measured from the horizontal. In a similar fashion as in the SEA, the instrument is pointing at the local horizontal at VEA of $0^{\circ}$, while it is pointing at the zenith direction at VEA $90^{\circ}$.

Both the VAA and the VEA vectors can vary during the day or depending on the location or measurement campaign. Their values have to be decided by the instrument 
operator. It is also worth noting that, throughout this work, both VEA and EVA (Elevation Viewing Angle) acronyms will be used indistinguishably for the elevation angle of the light collector.

\subsubsection{Air mass factor}

The air mass factor is the path length which light takes through the atmosphere normalized to the shortest possible path length (that is, when the Sun is directly overhead). The Air Mass Factor (AMF) quantifies the reduction in the power of light as it passes through the atmosphere and is absorbed by air molecules and particles. Assuming a coarse geometrical approximation, the overall AMF of the atmosphere can be estimated by Equation 3.9.

$$
A M F=1 / \cos (S Z A)
$$

The Equation 3.9 to calculate the $A M F$ assumes that the atmosphere is a flat horizontal layer. Although this idea is simple and not very accurate, the concept behind the AMF will be extremely useful when calculating light paths in Chapter 4 . The idea behind is: an $\mathrm{AMF}$ is the ratio between the actual photon path in a given atmospheric volume and the thickness of that volume.

\subsection{Measurements and dimensions}

The concentration of a trace gas is the amount of that particular trace gas per volumetric unit. It can be expressed either in terms of number of molecules (number density) or related to the mass (traditional -or mass- density). In atmospheric sciences, its units are typically [molecule $\mathrm{cm}^{-3}$ ] for number density and $\left[\mu \mathrm{g} \mathrm{m}^{-3}\right.$ ] for mass density.

However, there is another way of expressing concentration which is quite popular in this field: the mixing ratio. The mixing ratio is the relative amount of a trace gas compared 
to the amount of air at a given altitude. It can be defined in several different ways: number of moles of gas per mole of air [mol/mol], number density of a gas per number density of air [parts per] or equivalently in terms of volume of gas per volume of air $[\mathrm{v} / \mathrm{v}]$ since the volume occupied by an ideal gas is proportional to its number of molecules. Since it is a ratio between equivalent magnitudes, it has no dimensional units. Thus it is represented as "parts per", for instance parts per million [ppm], parts per billion [ppb] or parts per trillion [ppt] are commonly used. On a separate note, and again due to the fact of the mixing ratio being a relative magnitude, we will refer to it indistinctly as either "mixing ratio" or "relative concentration".

The mixing ratio of a gas has the virtue of remaining constant when the air density changes (as happens when the temperature or the pressure change). The mixing ratio is therefore a robust measure of atmospheric composition. It also offers another advantage: it allows us to fully assess vertical profiles and their evolution without having to compromise to offer absolute concentration values. Instead, the concept of mixing ratio is enough for our purposes. This will be particularly useful when dealing with the estimation of light paths, which can be rather complex (as will be discussed in Chapter 4): dividing the concentrations estimated from the absorptions by the air number density cancels out some of the uncertainties in deriving the light paths. 


\section{RADIATIVE TRANSFER}

This chapter describes the fundamentals on radiative transfer and its most important concepts, as well as the numerical calculations used in this thesis. The theoretical foundation of this chapter lies mainly in two sources: Solomon et al., 1987 and Rodgers, 2000.

\subsection{Introduction}

Ground, remote sensing techniques offer an effective way to carry out indirect measurements of atmospheric magnitudes (for instance vertical concentration profiles of trace gases) using instruments located on the ground. Moreover, some of these remote sensing instruments -like MAXDOAS instruments- are able to perform large spatial scale measurements. However, the main disadvantage of these remote measurements is that the measured variable often follows a complicated physical function. Here is where the inverse problem arises: a known system of equations (the forward model) describes the atmosphere along with its physical phenomena, hence we have to invert the measured absorptions in order to derive the atmospheric state. Frequently there is no analytical solution, and in most cases when there is, no unique mathematical solution can be obtained. Therefore, these inversion methods usually consist of optimal estimation techniques.

We apply inversion algorithms to the measured DSCDs to estimate the light paths and subsequently derive the trace gas vertical concentration profile. The main idea behind these inversion algorithms is based on the fact that each VEA has different scattering heights and light paths (Solomon et al., 1987). Therefore, a given set of measured DSCDs contains information about the vertical distribution of a certain trace gas. Since higher VEAs 
are generally related to higher scattering heights, different layers within the atmosphere can be sampled, especially in the lower troposphere. The study of the interaction between light and matter is extremely complex. Part of the problem comes from the atmosphere not being homogeneous: the density profiles and the overall composition change with altitude. Furthermore, some of the physical processes are difficult to describe mathematically, hence there is no easy way to simulate the entire atmosphere.

If we consider a small volume $(d V)$ throughout which we can safely assume homogeneity, the so-called Radiative Transfer Equation (RTE) describes mathematically the propagation of radiation through this $d V$ that may be affected by absorption, emission and scattering processes:

$$
\frac{d I_{\lambda}}{d s} * d a * d \Omega=j_{k} * d_{a} * d \Omega-\left(k_{\lambda}+\sigma_{\lambda}\right) I_{\lambda} * d a * d \Omega
$$

where $I_{\lambda}$ is the spectral radiance, $d_{s}$ and $d_{a}$ form the small volume $d V\left(d V=d a^{*} d s\right)$, and are -respectively- differentials of propagation and area. Furthermore, $j_{k}, k_{\lambda}, \sigma_{\lambda}$ are the emission, scattering and absorption spectral coefficients, respectively. $d \Omega$ is the solid angle differential. Thus simplifying the RTE, we obtain Equation 4.2.

$$
\frac{d I_{\lambda}}{d s}=j_{k} *-\left(k_{\lambda}+\sigma_{\lambda}\right) I_{\lambda}
$$

The RTE cannot be solved analytically except for the simplest, symmetrical, steady configurations (conditions that do not frequently occur within the atmosphere), hence numerical methods have to be applied in order to estimate the best solution, or at least optimize it. The algorithms that calculate these atmospheric events are called Radiative Transfer Models (RTMs), and they study the transport of radiation as well as its interaction with matter. There are several RTMs for atmospheric applications, and they can be divided into two main groups, depending on how they solve the RTE: i) deterministic methods such as the Discrete Ordinates method (Stamnes et al., 1988)- which discretize the entire 
atmosphere and generate discrete radiation fields and physical phenomena until an optimal solution with respect to the RTE is achieved; and ii) other RTMs are based on Monte Carlo methods, which estimate the radiation fields statistically (Deutschmann et al., 2011).

For this thesis, we have used the bePRO inversion algorithm, based on LIDORT discrete method- and developed at BIRA-IASB (Clémer et al., 2010).

\subsection{The forward model and its matrix notation}

The atmospheric RTMs often have complex calculations inside, therefore before describing each component mathematically, it is often helpful to relate the matrix general notation to get a first idea of how they work. The inversion of DSCD measurements to obtain information on the vertical distribution of the measured species, requires an accurate description of the physics of the measurement process. This forward model should include all the physics of the measurements to an accuracy that is better than that of the measurements. In practice, the complete physics of the measurement is not fully understood or it is so complicated that it must be approximated by the forward model $F(\vec{x}, b)$, modified by the experimental uncertainty:

$$
\vec{y}=F(\vec{x}, b)+\vec{\varepsilon}
$$

where $\vec{y}$ is the vector of measurements (DSCDs in the case of MAXDOAS) with uncertainty $\vec{\varepsilon}, \vec{x}$ is the state vector containing the vertical distribution of the trace gas or aerosols being measured, and $b$ stands for forward model parameters that influence the modelled DSCDs, but are not retrieved. Since the measurements are discrete quantities,

while the vertical distribution to be retrieved, $\vec{x}$, is in principle a continuous function, the problem is formally ill-posed or underconstrained. Therefore, the state vector is discretized into a finite number of components depending on the resolution required and the information content of the measurement. 
The input to the radiative transfer model includes the state profile $\vec{x}$, and the forward model parameters $b$ such as temperature, pressure, ozone and aerosol profiles. The radiative transfer model then calculates the forward model DSCDs via the weighting functions $(K)$. The weighting function matrix $(K)$ gives the sensitivity of the modelled DSCD to changes in each discrete layer ( $n$ ) of the state profile. It may also be called the Jacobian, since it is a matrix of derivatives, the kernel (hence $K$ ), or the adjoin (Equation 4.4).

$$
K=\frac{d F(\vec{x})}{d b}
$$

More concretely, the matrix $K$ is defined as the sensitivity of the forward model to the forward model parameters. Focusing on our goals (retrieving the measured DSCDs), $K$ can be interpreted as the matrix that ultimately converts the vertical concentration profiles of a given trace gas into column densities, hence $K$ actually stands for the box-AMFs (the AMF of each atmospheric layer). The sensitivity of these box-AMFs and consequently DSCDs to several forward model parameters, including SZA, RAA, VEA, aerosol extinction profile, Henvey-Greenstein asymmetry parameter or surface albedo are included inside $K$.

By far the most time-consuming part of the radiative transfer calculation is the determination of box-AMFs. For a linear inversion, the sensitivity of the measurements to the state must be independent of the state vector itself and the forward model can then be described in terms of the constant weighting function matrix, $K$ :

$$
\vec{y}=K \vec{x}+\vec{\varepsilon}
$$

Trace gases measured by MAXDOAS are, by requirement of the technique, optically thin $(\tau<<1)$ and so the linear Maximum A Posteriori (MAP) retrieval method can be safely applied. Optically thick absorbers and aerosols influence the radiance and hence the DSCDs in a non-linear way with respect to density, so an iterative numerical solution such as a Gauss-Newtonian method must be used (Wedderburn, 1974) in those cases. 
Given a set of measurements $\vec{y}$ with their uncertainty covariance, the forward model $F(\vec{x})$, and any available a priori information, the possible states $\vec{x}$ consistent with this information can be assigned a Probability Density Function (PDF). The MAP optimal estimation method chooses the state that maximises the posterior conditional PDF $P(x / y)$, meaning that $P(x / y) d x$ is the probability that $\mathrm{x}$ is in the interval $[x, x+d x]$ for a given set of measurements $\vec{y}$. In contrast, the Maximum Likelihood (ML) solution maximises the conditional $\operatorname{PDF} P(x / y)$.

The linear maximum a posterior solution $(\hat{x})$ can be written as Equation 4.6.

$$
\hat{x}=\vec{x}_{a}+G\left(\vec{y}-K \vec{x}_{a}\right)
$$

Where $\vec{x}_{a}$ is the a priori profile and $G$ stands for the gain matrix. The gain matrix, or contribution function matrix, $G$, quantifies the contribution of each DSCD measurement to the retrieved profile, or in other words the sensitivity of the retrieval to the measurement.

Optimal estimation ideally chooses the best solution from all possible solutions that minimize the difference between the measured and modelled vectors based on the measurements, their uncertainties and the prior constraints. In order to understand and correctly interpret the retrieved state profile, it is important to understand how the solution is related to the "true" state and to understand the various sources of uncertainty that propagate into the retrieval.

Another important concept is the averaging kernel matrix, $A$. It describes the sensitivity of the retrieval to the "true" state and is the product of the gain and weighting function matrices, Equation 4.7.

$$
A=G K
$$


This matrix indicates how a certain layer is sensible to surrounding layers.

\subsection{Aerosol extinction and trace gas profile retrieval}

The original calculation was built based on the Optimal Estimation Method (OEM; Rodgers, 2000) and it comprises two steps: first, the light paths and the vertical profiles of irradiance extinction are calculated using the $\mathrm{O}_{4}$ DSCDs; then, the target trace gas vertical concentration profile is retrieved using the corresponding light paths and measured absorptions. In order to do that, bePRO simulates the atmospheric state characterizing several different physical phenomena including pressure and temperature vertical profiles, Rayleigh and Mie scattering events (along with their respective phase functions), the effect of the surface albedo, the light path geometries or the irradiance extinction processes. Once the atmospheric vector state is defined, its combination with a certain vertical concentration profile results in the simulated DSCDs. This vertical profile is iterated until the generated set of simulated DSCDs is optimized with respect to the measured DSCDs so that the residual is minimized. As a result, an optimal vertical profile is obtained when the iteration is finished for each MAXDOAS cycle.

$$
X^{2}(x)=\sum_{m=0}^{M}\left(\frac{F_{m}(\vec{x})-y_{m}}{\sigma_{m}}\right)^{2}+\sum_{n=0}^{N}\left(\frac{x_{n}-x_{a, n}}{\sigma_{a, n}}\right)^{2}
$$

This equation is divided into two parts. One is based on the difference between the modelled $F_{m}(\vec{x})$ and measured values $\left(y_{m}\right)$ and the second represents the discrepancy between a certain atmospheric state vector $\left(x_{n}\right)$ and an a priori profile $\left(x_{a, n}\right)$. Each term is weighted by their respective uncertainties $\left(\sigma_{m}, \sigma_{a, n}\right)$. The method finds (by iterations) one particular solution from an infinite range of possible solutions that is statistically optimal $\left(X^{2}(x) \min \right)$.

The measured $\mathrm{O}_{4}$ DSCDs are used to estimate the light paths for each VEA since they are related to the square of the atmospheric $\mathrm{O}_{2}$ profiles, which are well-known. This profile 
is fairly steady during the day and does not heavily depend on chemistry. Therefore, the measured $\mathrm{O}_{4}$ DSCDs can provide information on the irradiance extinction in the atmosphere. This extinction profile, retrieved using bePRO, is usually associated with the aerosol extinction coefficients and thus, its vertical integration yields the AOD. These aerosol extinction profiles are required to subsequently evaluate trace gas profiles since they strongly affect the relative light paths and hence the concentration profiles derived from them.

Once the light paths are computed in the previous step, a linear fit is carried out for the measured DSCDs of the target trace gas, yielding the optimal vertical concentration profile. The vertical integration of this concentration profile is called the vertical column density.

\subsubsection{A priori profiles}

The inversion algorithm simulates the atmosphere along with the corresponding parameters included, such as temperature and pressure profiles, irradiance levels for each height layer, scattering evaluation, aerosol loading, light path geometry and vertical concentration profiles of absorbers. In order to perform the retrieval, which is a non-linear, iterative system of equations with non-unique solution, an a priori profile is needed, both for starting the iteration process and to prevent the final solution to be not realistic enough.

We have thus to build a priori profiles for the aerosol extinction and trace gas retrievals. For both cases we chose exponentially decreasing profiles:

$$
c_{a}(z)=\left(\frac{V C_{a}}{s h}\right) * \exp \left(\frac{-z}{s h}\right)
$$

where $c_{a}(z)$ is the a priori profile for the MAXDOAS cycle at a given altitude $\mathrm{z}$, sh is the scaling height constant and $V C_{a}$ is the vertical column of the associated amount. We assumed $0.5 \mathrm{~km}$ as scaling height constant for all the a priori profiles and a fixed total 
vertical amount: for the aerosol retrieval we took the AOD from AERONET while for trace gases we applied the geometrical approximation to the measured DSCDs at VEA $30^{\circ}$. This approach is founded on the idea that the trace gas is supposed to be located below the scattering height, so that geometrical considerations can be implemented for the purpose of estimating the air mass factor and hence derive the vertical column (Hönninger et al., 2004). This approach allows us to take the possible diurnal variability of the a priori profiles into account with simple, straightforward calculations.

This approach also allows the use of different a priori profiles for each MAXDOAS vertical scan and each trace gas. The height grid used in this work had 72 layers, the first one ranging from $0 \mathrm{~m}$ (ground surface) to $100 \mathrm{~m}$; then nine layers of $100 \mathrm{~m}$ thickness each, between $0.1 \mathrm{~km}$ and $1 \mathrm{~km}$; after that, we included another fifteen layers ranging from $1 \mathrm{~km}$ to $4 \mathrm{~km}$ of $200 \mathrm{~m}$ thickness each; the twelve layers of $500 \mathrm{~m}$ thickness from $4 \mathrm{~km}$ to $10 \mathrm{~km}$, from $10 \mathrm{~km}$ to $30 \mathrm{~km}$ the layers have $1 \mathrm{~km}$ and finally the remaining layers have $2 \mathrm{~km}$ thickness (up to $60 \mathrm{~km}$ height). For the bePRO retrievals, the temperature and pressure profiles were taken from the U.S. Standard Atmosphere files and interpolated to Madrid height above sea level $(667 \mathrm{~m})$ and the height grid described in Chapters 6 and 7. While the U.S. Standard Atmosphere profiles were used in Chapter 8.

\subsection{Atmospheric processes}

\subsubsection{In-scattering and out-scattering}

In a general way, scattering can be defined as the process in which a particle diffuses incoming radiation at a given angle and with certain energy. If the energy is conserved, the scattering is called elastic, whereas if the energy changes, the scattering is called inelastic. Concerning the atmosphere, light can be scattered once or more times. Given that in the MAXDOAS technique we are dealing with scattered sunlight, we can safely assume that most of the collected irradiance by the collector will be single-scattered. Multiple scattering 
indeed has a noticeable effect, and will be taken care of, but can be considered as a secondorder contribution.

For now, we can just assume that all of the irradiance that arrives at the MAXDOAS instrument comes from a single scattering event, hence previous to that single scattering event the light must be direct sunlight. At the top of the atmosphere, all of the irradiance is maximum. From that point down to the single scattering event, as light traverses the atmosphere the irradiance decreases due to absorption and scattering processes. The portion of light that is scattered out of the way of the light beam is called out-scattered. After the scattering process, another portion of light is out-scattered in its way to the light collector. Therefore, the irradiance that reaches the instrument will always be lower than that at the top of the atmosphere.

On the other hand, we will refer to as in-scattering when light that originally was not in the line of sight of the instrument is eventually scattered into the angular direction of the MAXDOAS light collector. Under certain conditions, in-scattering allows seeing light sources that are not in the detector line of sight. Its most obvious optical effect results in light halos around light sources.

An atmospheric scattering event depends on the probability of light reaching air molecules, therefore it is conceptually pleasing to think that, the denser the medium, the more likely the scattering event will occur. Atmospheric scattering is also related to light paths and hence the distance travelled by light. The larger the distance, the higher the number of scattering events.

In order to quantify this process, the concept of the scattering function $S$ will be used. Its purpose is to derive how much light is scattered in a certain direction for a given wavelength. 
$I_{f}(\lambda)=I_{0}(\lambda) * S(\lambda, \theta, z)$

where $S(\lambda, \theta, z)$ depends on wavelength, altitude and relative scattering angle.

\subsubsection{Rayleigh scattering}

Rayleigh scattering -named after the British physicist Lord Rayleigh- models how light is scattered by air molecules (which are relatively small), mainly $\mathrm{N}_{2}$ and $\mathrm{O}_{2}$. When irradiance reaches a small particle, some of it will continue unaffected, but a portion of the incoming light will interact with the particle, ending up being scattered in all directions. Nonetheless, not all of the possible angular directions receive the same amount of energy. Figure 4.1 shows a simple diagram of a Rayleigh scattering event. The basic equation that describes the Rayleigh scattering function is in Equation 4.11.

$$
S(\lambda, \theta, z)=\frac{\pi^{2}\left(n^{2}-1\right)^{2} \rho(z)\left(1+\cos ^{2} \theta\right)}{2 N \lambda^{4}}
$$

where $\lambda$ is the radiation wavelength, $\theta$ is the angular deviation from the scattered light with respect to the original direction of light and $z$ represents the altitude from the ground. Moreover, $n$ stands for the refractive index of air, $\rho$ represents the density ratio which will be described below- and $N$ is the mean surface molecular number density of air. We can observe the famous $\lambda^{-4}$ dependence of the Rayleigh scattering with respect to the incoming wavelength.

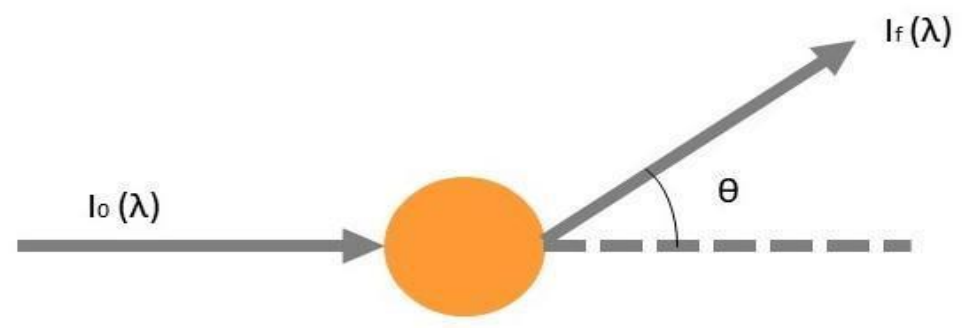

Figure 4.1. Basic diagram of a light scattering event. 
Note from the Equation 4.9 that the Rayleigh-scattered light is not homogeneous in $\theta$, on the contrary, some directions receive more light than others. Figure 4.2 shows the usual eight-shape of the Rayleigh function, as well as the strong relationship with wavelength.

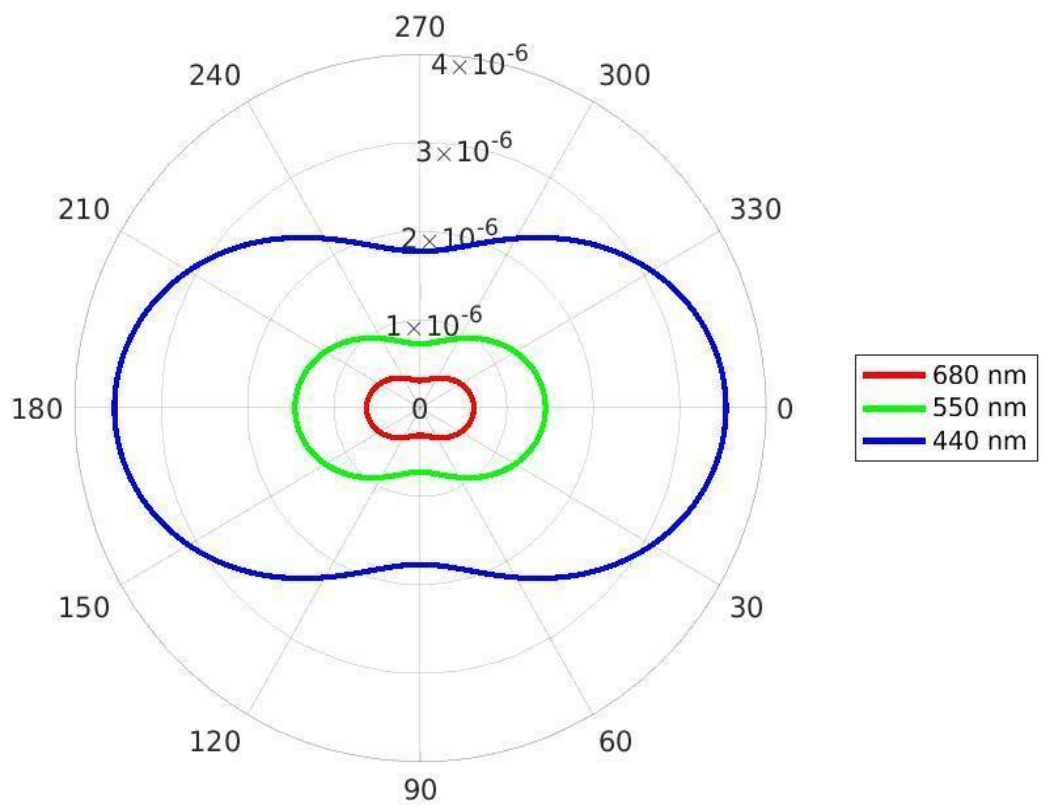

Figure 4.2. Rayleigh function with respect to wavelength.

Although it may seem intuitively easy to think of the Rayleigh scattering as light "bouncing off" air molecules, that is not really the case. Light consists of an electromagnetic wave, and it can interact with the charge imbalances that are naturally present in certain molecules. Those charges become excited by the absorption of the incoming electromagnetic radiation, which is later re-emitted. The two-lobed shape you see in the phase function shows that air molecules become electric dipoles, radiating exactly like microscopic antennas.

Figure 4.2 shows that the angular preferences of photons after a Rayleigh scattering are either continuing straight through unaffected, or going back at $180^{\circ}$, whereas the less probable outcome is being scattered at 90 degrees. The equation of the Rayleigh scattering 
function indicates how much light is scattered towards a particular direction. However, it gives no direct information about how much energy is scattered in total. Integrating Equation 4.11 over the three-dimensional solid angle, and normalizing so that the integral over all possible angles sums 1, the Rayleigh scattering coefficient is obtained in Equation 4.12.

$$
\beta(\lambda, z)=\frac{8 \pi^{3}\left(n^{2}-1\right)^{2}}{3} \frac{\rho(z)}{N} \frac{1}{\lambda^{4}}
$$

where $\beta(\lambda, z)$ stands for the fraction of total energy which is lost after a single scattering event. Figure 4.3 includes the evolution of the Rayleigh scattering coefficient as a function of wavelength.

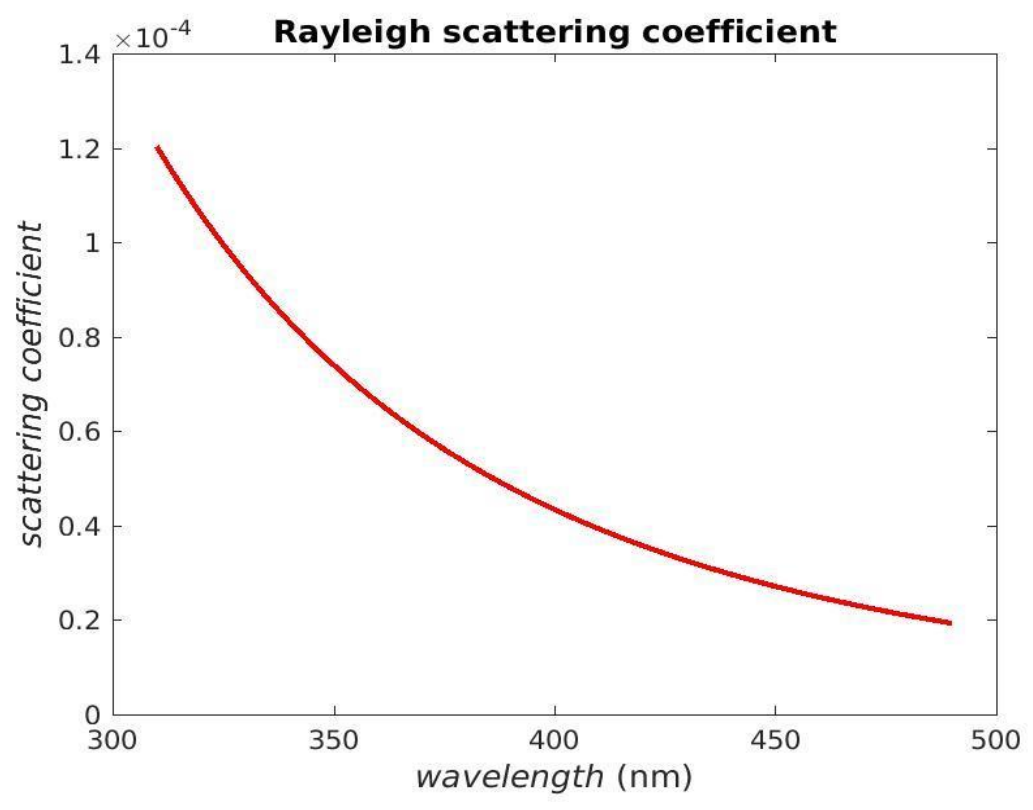

Figure 4.3. Rayleigh scattering coefficient at different wavelengths.

The strong relationship between wavelength and the Rayleigh scattering coefficient is what ultimately makes the sky blue and the sunrises and sunsets red. As can be observed in Figure 4.3, bluish light (shorter wavelength) is heavily scattered in comparison with reddish light (larger wavelength). This means that, in the early morning and in the evening, 
when the Sun is at low elevation angles -more horizontal, longer light paths- most of the bluish light has been out-scattered when it reaches our eyes, thus the reddish color. On the other hand, during the day, when the SZA is lower, wherever we look at the sky -barring direct sunlight- what we see is basically scattered sunlight, and since bluish light is inscattered considerably more that reddish light, hence the usual blue-sky color that we observe.

Regarding the Rayleigh scattering coefficient, we can decompose the Rayleigh scattering function $S$ into two components: the Rayleigh scattering coefficient $B(\lambda, z)$ described above, which modulates the intensity, and a component in charge of the angular direction. This last component can be derived by dividing Equation 4.11 and Equation 4.12:

$$
\gamma(\theta)=\frac{3}{16 \pi}\left(1+\cos ^{2} \theta\right)
$$

Note that $\gamma(\theta)$ does not depend on incoming light wavelength, as we could expect. This equation is responsible for the dipole shape in Figure 4.2.

\subsubsection{Mie scattering}

Rayleigh scattering works well when dealing with particles (like atoms or air molecules) that are small in comparison to the light wavelength. However, it does fail describing the scattering by larger particles. Since the amount of these larger particles in the atmosphere is not negligible, it is required to somehow introduce an additional scattering term in order to mathematically describe the atmosphere in a more comprehensive way.

Mie scattering was first described in a fully manner way by Gustav Mie and is defined as the interaction of light with (particulate) matter of dimensions comparable to the wavelength of the incident radiation (Mie, 1908). Since the linear dimension of the particle is comparable to the wavelength of the radiation, interference effects occur. The 
most noticeable difference compared to Rayleigh scattering is the typically much weaker wavelength dependence (usually proportional to $\lambda^{-1.3}$ ) and a strong dominance of the forward direction in the scattered light. Since Mie scattering involves solving Maxwell's equations, the calculation of the Mie scattering spectral cross section can be complicated (involving summing over slowly converging series), even for spherical particles, but even more so for particles of arbitrary shape. Therefore, the Mie scattering contribution can only be approximated.

In an analogous way as with the Rayleigh scattering, the Mie scattering depends on the relative angular direction of the scattered light with respect to the incoming light. Although it may be very complicated, there are several numerical approximations that yield very good results. One of the most common is the Henyey-Greenstein function (Henyey and Greenstein, 1941), firstly derived in 1941.

$$
\gamma_{M}(\theta)=\frac{3}{8 \pi} \frac{1-g^{2}}{2+g^{2}} \frac{1+\cos ^{2} \theta}{\left(1+g^{2}-2 g \cos \theta\right)^{3 / 2}}
$$

where the parameter $g$ (which ranges from -1 to 1 ) is referred to as the preferred scattering direction, and controls the anisotropy of the Mie scattering. Figure 4.4 includes three examples of the Mie scattering phase function.

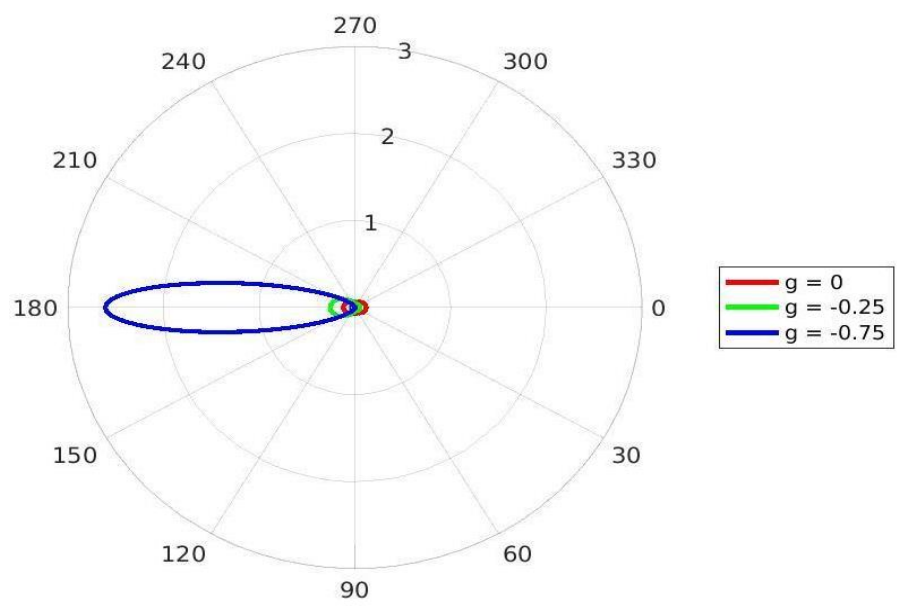

Figure 4.4. Mie scattering phase function for different values of the parameter $\mathrm{g}$. 
The value of $g$ varies depending on factors such as the environment and aerosol loading. In urban, polluted environments, for instance, Mie scattering is much stronger than in natural regions. While Rayleigh scattering is responsible for blue skies and red sunsets, Mie scattering is what causes clouds to be white.

\subsubsection{Atmospheric density}

As previously discussed (see Subsection 4.4.1), atmospheric scattering is proportional to the atmospheric density. A denser medium means more chances for the scattering events to occur. The parameter $\rho(z)$ represents the normalized atmospheric density as a function of altitude:

$$
\rho(z)=\frac{n_{\text {air }}(z)}{n_{\text {air }}(0)}
$$

here $n_{\text {air }}(z)$ stands for the atmospheric number density at a given altitude $z$. Using the Ideal Gas Law (pressures in the atmosphere are sufficiently low that this law holds to an estimate accuracy of about $1 \%$ ) along with the temperature and pressure vertical profiles, the atmospheric density vector can be derived in Equation 4.16.

$$
n_{\text {air }}(z)=\frac{P(z) N_{A}}{R T(z)}
$$

where $P(z)$ and $T(z)$ are the pressure and temperature values at altitude $z, N_{A}$ is the Avogadro number and $R$ is the ideal gas constant. Figure 4.5 includes the number density profile for the U.S. Standard Atmosphere: 


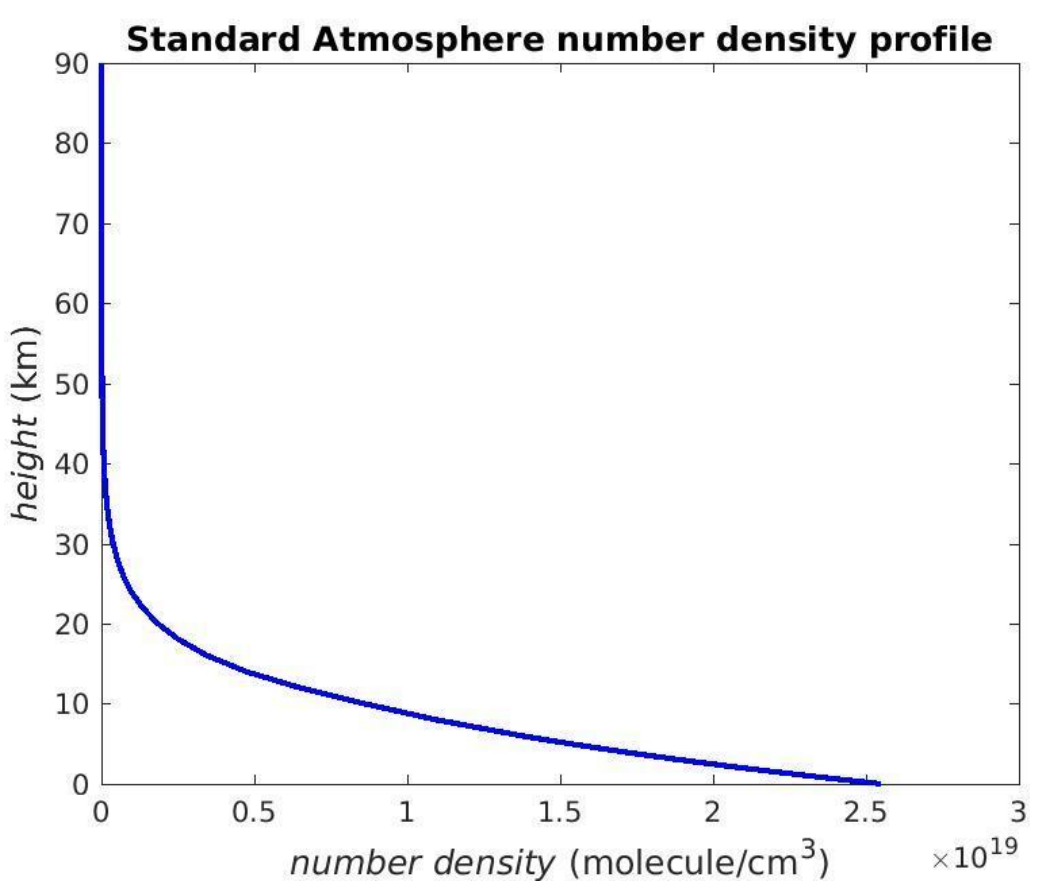

Figure 4.5. U.S. Standard Atmosphere number density profile.

\subsubsection{Aerosol extinction}

Another way of irradiance extinction comes from aerosols. As mentioned before (Section 4.3), the aerosol loading has a huge impact on the light paths, and hence on the profile retrieval, specially for lower altitudes. This means that the aerosol vertical profile is required. When using MAXDOAS measurements, these profiles are not readily available, nor they can be directly inferred. Instead, they have to be optimised within a non-linear fitting so that the simulated $\mathrm{O}_{4}$ absorptions match the measured ones. Once the extinction profile by aerosols is estimated, it can be included along the out-scattering extinction terms.

\subsubsection{Integrating over light paths}

In the previous subsections, we have explained how to numerically describe single scattering events in the atmosphere. The scattering coefficients represent the fraction of irradiance that is lost in a single scattering interaction. Hence the light that continues unaffected would be: 


$$
I_{f}(\lambda)=I_{0}(\lambda)-I_{0}(\lambda) ß(\lambda)
$$

where $I_{0}(\lambda)$ and $I_{f}(\lambda)$ are, respectively, the initial and final spectral irradiance. While this holds for a single collision, we are interested in understanding how much energy is scattered over a certain distance. This means that, at each point, the remaining light is subject to this process all over again. When light travels through a differential volume $d V$ considering a uniform medium- with a given scattering coefficient, the final irradiance that gets out of $d V$ is shown in Equation 4.18.

$$
I_{f}(\lambda)=I_{0}(\lambda) * \exp (-\beta * s)
$$

and here $\beta$ represents all of the scattering coefficients and $s$ the distance travelled by light within $d V$.

Therefore, here we can already simulate the irradiance that would arrive at the MAXDOAS instrument considering all of the possible scattering events. In order to do that, a discrete three-dimensional grid has to be defined, generating cells of $d V$ where light propagates through. There has to be a compromise in the size of these differential volumes, since they have to be large enough to account for a statistically significant amount of processes, but not to an extent to which the descriptive coefficients can no longer be regarded as constant inside $d V$.

The individual contribution to the scattered radiation by an infinitesimally thin layer at altitude $z$ is given by:

$$
I_{z, s}=I_{z} S_{R} * n_{\text {air }}(z) * E F * \Delta z
$$

where $I_{Z}$ is the radiation reaching the layer located at altitude $z, S_{R}$ represents the overall Rayleigh scattering function, $\Delta z$ is the thickness of the aforementioned infinitesimal 
layer and $E F$ stands for the Enhancement Factor, i.e. how much longer is the actual photon path traversing the volume $d V$ in relation to its thickness $\Delta z$.

Implementing these equations and summing over all of the cells, a vector of contributing intensities is estimated: each layer contributes with a relative irradiance to the total amount received at the light collector. Since we are dealing with relative intensities, a probability vector can be calculated normalising the contribution intensities vector to one. This probability vector can be interpreted as the relative probability of a given collected photon to come from a certain layer.

\subsubsection{Scattering weights}

As detailed in Chapter 3, the AMF is a concept that portrays the relationship between the thickness of the atmosphere and the actual light paths. However, we have seen that the collected photons do not follow an unique path, instead the registered spectrum consists of an ensemble of photons, each coming from different light paths. This discrete method characterizes numerically every possible light path. Since the scattering events can occur at any layer, different light paths need to be accounted for. This is the reason why the idea of box-AMFs is introduced: they represent the same as the general AMF but for every layer. Figure 4.6 includes a diagram of the observation geometry of a MAXDOAS instrument. 


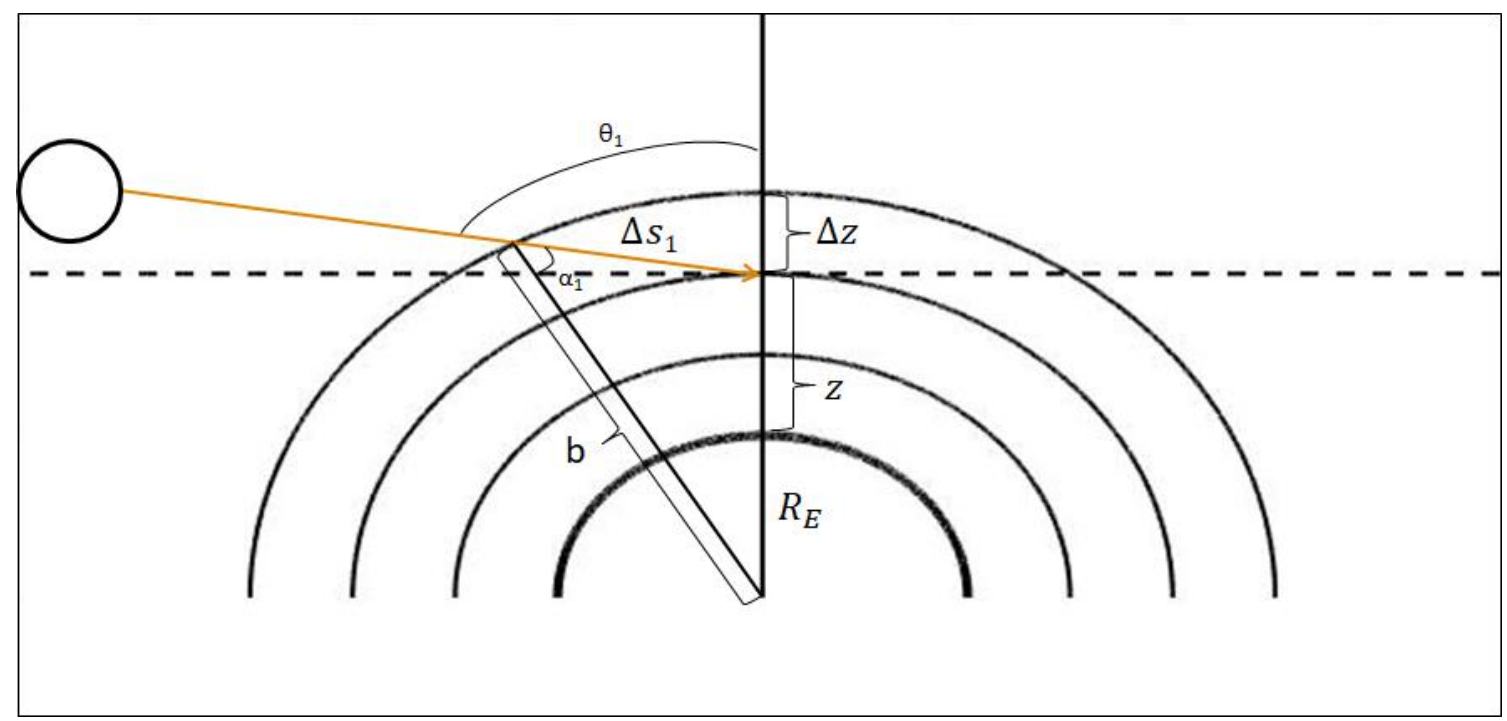

Figure 4.6. Geometry for a given SZA $\Theta$

The box-AMF for a layer $i$ is defined as in Equation 4.20.

$$
b o x-A M F_{i}=\frac{\Delta s_{i}}{\Delta z_{i}}
$$

here $\Delta s_{i}$ is the actual photon path inside layer $i$, whilst $\Delta z_{i}$ is the $i$-layer thickness. Multiplying the probability vector times the box-AMF vector the so-called scattering weights are calculated. They symbolize the contribution of a layer $i$ to the absorptions of a given species (Equation 4.21).

$$
\text { scd }=S W * \text { profile }
$$

Hence the differential scattering weights of two observational directions result in the desired matrix $K$ that was described in Section 4.2. 


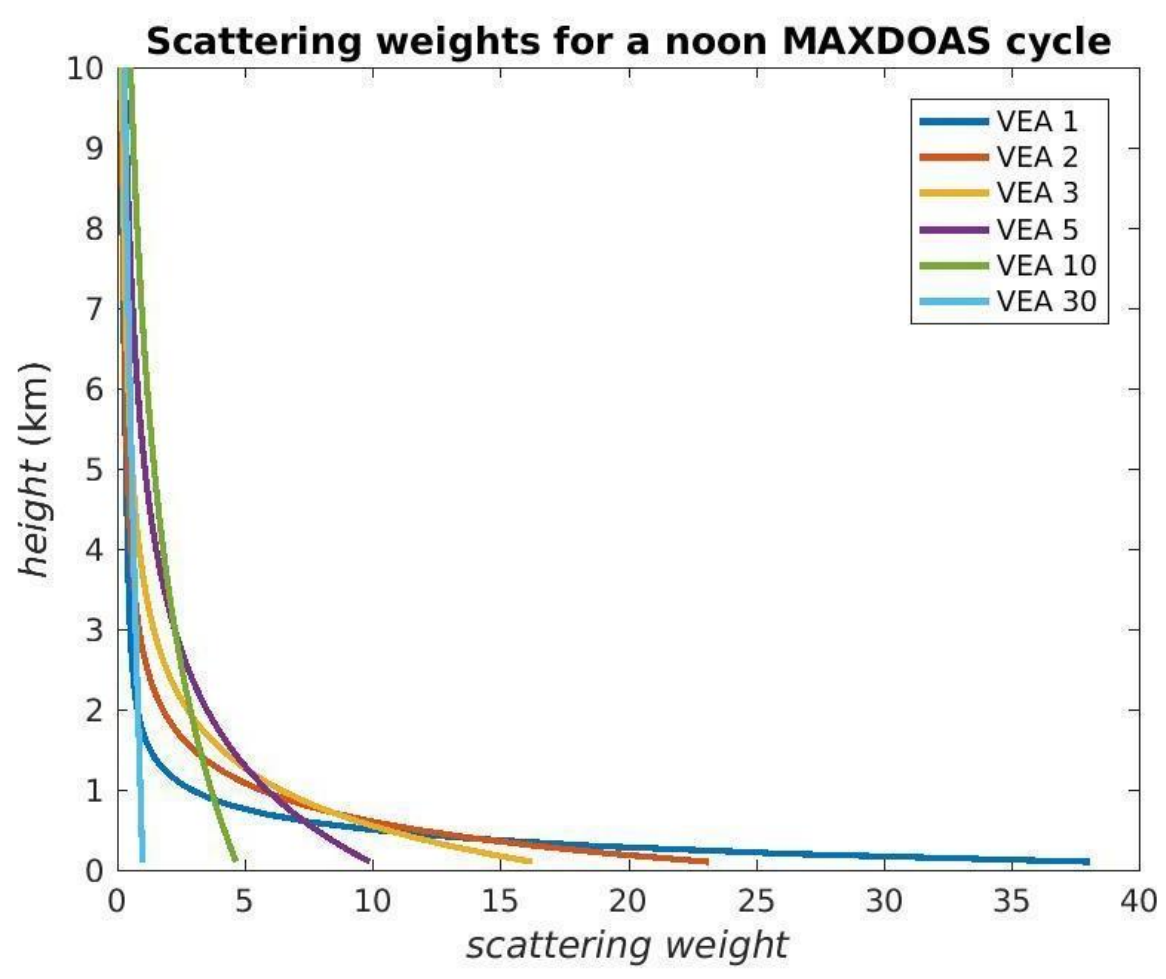

Figure 4.7. Scattering weights for different VEA.

\subsubsection{Multiple scattering and albedo}

Once the single scattering events are evaluated, the multiple scattering contribution can be estimated by letting single, out-scattered photons to be in-scattered again into the light collector line of sight. Since the probability of a scattering event (determined by the scattering coefficients) is already relatively low, and a multiple scattering event involves several scatterings, the scattering probability follows an exponential function, thus largely decreasing the contribution of high-order scatterings. Hence only multiple scattering of second order is usually implemented. Note, though, that there are several studies which suggest that the higher-order multiple scatterings contribution is negligible (Gomez et al., 2014).

Additionally, the albedo can be mathematically regarded as a multiple scattering event with respect to the surface. 


\subsection{Cloud screening}

MAXDOAS profile retrievals of aerosols and trace gases typically assume clear-sky conditions in the forward model. Actually, these measurements can be strongly affected by clouds, mainly due to multiple scattering effects, leading to significant data quality degradation and larger uncertainties on the retrievals. For this reason, the cloudcontaminated measurements have to be somehow flagged and excluded. It is required to apply a could index to the entire database.

Within this work, we apply a cloud index to the entire database. In order to do this, we used the AERONET database. The AERONET program is a global network of groundbased aerosol remote sensing instruments established by NASA and PHOTONS (http://aeronet.gsfc.nasa.gov). It provides a long-term, continuous and readily accessible public domain database of aerosol optical, microphysical and radiative properties for aerosol research. AERONET has three data quality levels: we used their latest Version $3.0-$ Level 1.5 (which is cloud-screened).

\subsection{Uncertainties}

The precision of the MAXDOAS calculations comprises two main contributions:

- The uncertainties from the DOAS analysis.

- The uncertainties of the profile retrieval.

Assuming they are independent, the overall uncertainty of the analysis is estimated propagating these two uncertainty sources. It is worth noting that the absorption cross sections of the trace gases also have uncertainties, sometimes quite noticeable. Nonetheless, for the MAXDOAS analysis, the cross sections are assumed as tabulated constants. However, they still have uncertainties, which will be taken into account in the overall propagation of uncertainties. 


\subsection{Detection limit and quality filters}

We estimate the minimum detectable optical density as Equation 4.22.

$$
O D_{\min }=\frac{R_{o p t m}}{\sigma_{\max }^{\prime}}
$$

where $O D_{\min }$ is the estimated minimum optical density that we can measure, $R_{\text {optm }}$ represents the RMS of the optimal residual (in optical density units) from our DOAS analysis and $\sigma_{\max }^{\prime}$ stands for the maximum differential absorption cross section of a given trace gas.

Once the DSCDs were obtained for each measurement campaign, and since they came from a system of nonlinear, least-squares equations, there was a gradient of quality and uncertainties in the results. Therefore, before using the profile algorithm, we applied a quality filter to the DSCDs. For doing this, we discarded every DSCD that had either a residual Root Mean Square (RMS) > 0.01 (in optical density units) or a relative uncertainty higher than 1. We also filtered out the DSCDs with an absolute value lower than twice the estimated detection limit, assuming it to be, for each day, the lower residual divided by the maximum value of the respective differential absorption cross section as described by Equation 4.20 (see Peters et al., 2012 for more details). 


\section{EXPERIMENTAL}

This chapter describes the equipment used for the measurements of this thesis. That includes urban measurements performed in Madrid, Spain, using MAXDOAS instruments with both $1 \mathrm{D}$ and $2 \mathrm{D}$ angular movements. Each instrument will be fully described, as well as the location and measurements set up. Finally, common parameters of the measurements will be detailed, along with the wavelength calibration procedure.

\subsection{Urban measurements site}

Our urban MAXDOAS instruments are located on the roof of the Instituto de Ciencias Agrarias (ICA) -within the main campus of the Spanish National Research Council (CSIC) in Madrid- at a latitude of $40^{\circ} 26^{\prime} 30^{\prime \prime} \mathrm{N}$ and a longitude of $3^{\circ} 41^{\prime} 15^{\prime \prime} \mathrm{W}$. The height of the building is approximately $70 \mathrm{~m}$ above the mean ground level of Madrid. Madrid is the biggest city in Spain, with over 3 million inhabitants. It is located in the centre of the Iberian Peninsula, the city is bordered (in the north-northwest) by the Sierra de Guadarrama, which is a mountain range of about $2400 \mathrm{~m}$ high. Moreover, Madrid has an area with low mountains in the east and northeast. The measurements were taken in the downtown area of Madrid, a location that can be classified as an urban site, with the usual weather of continental areas at mid-latitudes (i.e. hot and dry summers and cold winters), and with prevalence of clear sky days during the year. On average, precipitation occurs 63 days per year approximately and the mean relative humidity is about $57 \%$.

Madrid suffers from severe traffic congestion throughout the city with considerable emissions from house heating and factories. For these reasons, Madrid has endured episodes of air pollution by several compounds such as $\mathrm{NO}_{2}$ or $\mathrm{O}_{3}$ (Madrid's Air Quality 
Plan, 2019). Figure 5.1 shows the location of the instrument inside the city along with the VAA of the MAXDOAS-1D (which will be described in the following section).

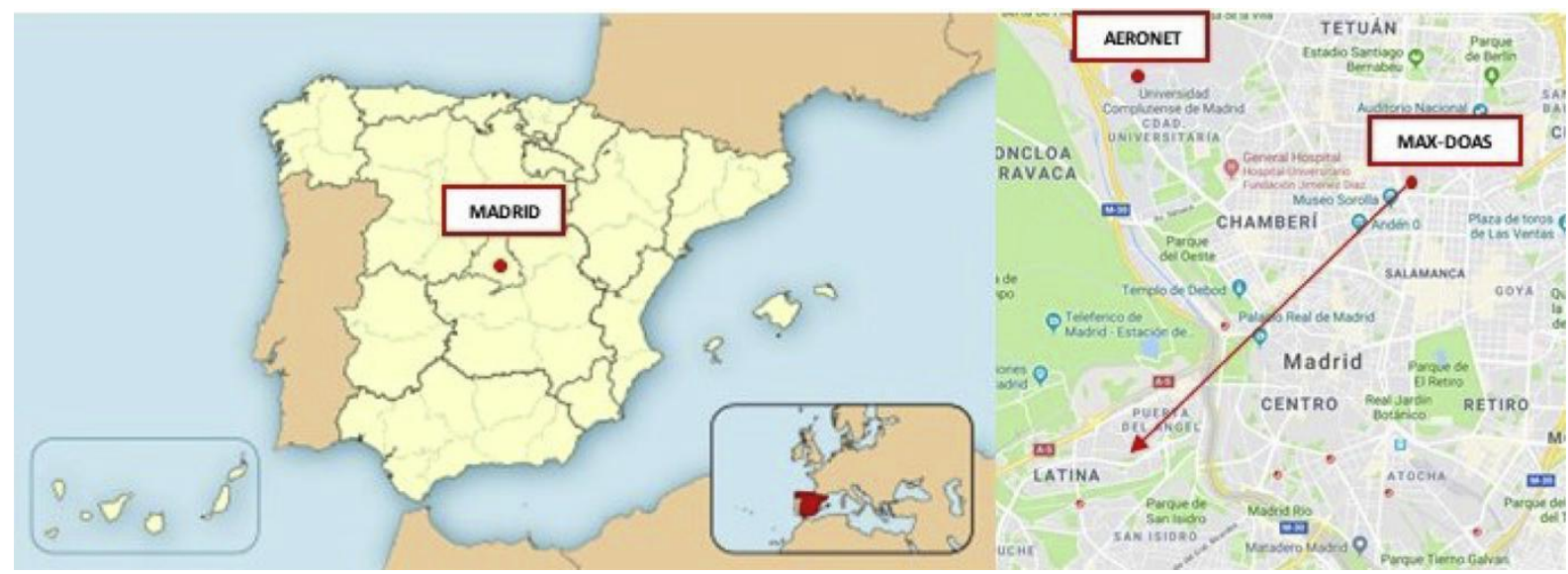

Figure 5.1: MAXDOAS-1D location in Madrid (Spain).

\subsection{Description of the MAXDOAS-1D instrument}

Our MAXDOAS-1D instrument (shown in Figure 5.2) was installed in 2014 and has been in operation continuously since then. It has two main subsystems (fully described in Prados-Roman et al., 2015 and Wang et al., 2016). Briefly, the external sub-system is placed on the ICA building roof and it consists of a telescope which receives sunlight that is scattered in the atmosphere at different altitudes. The light is focused by a lens (with 200 $\mathrm{mm}$ of focal distance) with a diameter of $50.8 \mathrm{~mm}$ and then, it goes through an optical fiber which has 19 individual quartz fibers. The optical fiber is connected with the internal part, which comprises a spectrometer (Princeton Instruments SP500i), a Charge-Coupled Device detector (camera CCD Princeton Instruments Pixis 400B) and a computer. This CCD sensor is cooled down to $-70^{\circ} \mathrm{C}$ thus making the influence of the dark current negligible. Once in the spectrometer, the light is diffracted and dispersed by a 600 groove/mm grating before reaching the CCD sensor. All of the components are connected to a computer that saves the data and keeps the instrument running. The internal sub-system is placed in a temperature-controlled room. Instrumental effects such as the electronic offset and the dark current are considered and subtracted from each measured spectrum using the 
equations derived in Section 5.4. The exposure time is automatically calculated in order to optimize the light input, hence depending on the sunlight intensity (Benavent et al., 2019).

As for the measurements set-up, the light collector accumulates the scattered sunlight with the following VEAs sequence: $0,1,2,3,5,7,10,15,20,30$ and $90^{\circ}$, for each MAXDOAS vertical cycle. The data were acquired at these elevation angles for about $30 \mathrm{~s}$ of total exposure time in each angle, taking between 2 and 50 measurements within each position depending on the sunlight intensity and with the purpose of maximizing the signalto-noise ratio. The instrument operates continuously up to $95^{\circ}$ of SZA, although only measurements below $90^{\circ} \mathrm{SZA}$ have been considered for this work. The rotating grating of the spectrometer allows the analysis in the UV region (305-394 $\mathrm{nm}$ ) and in the VIS range (406-493 nm) with a spectral resolution of $0.5 \mathrm{~nm}$ of FWHM over the full spectral window. The telescope is pointing to the south-southwest direction: VAA of $212^{\circ}$ (measured eastwards from the north, see Figure 5.1).

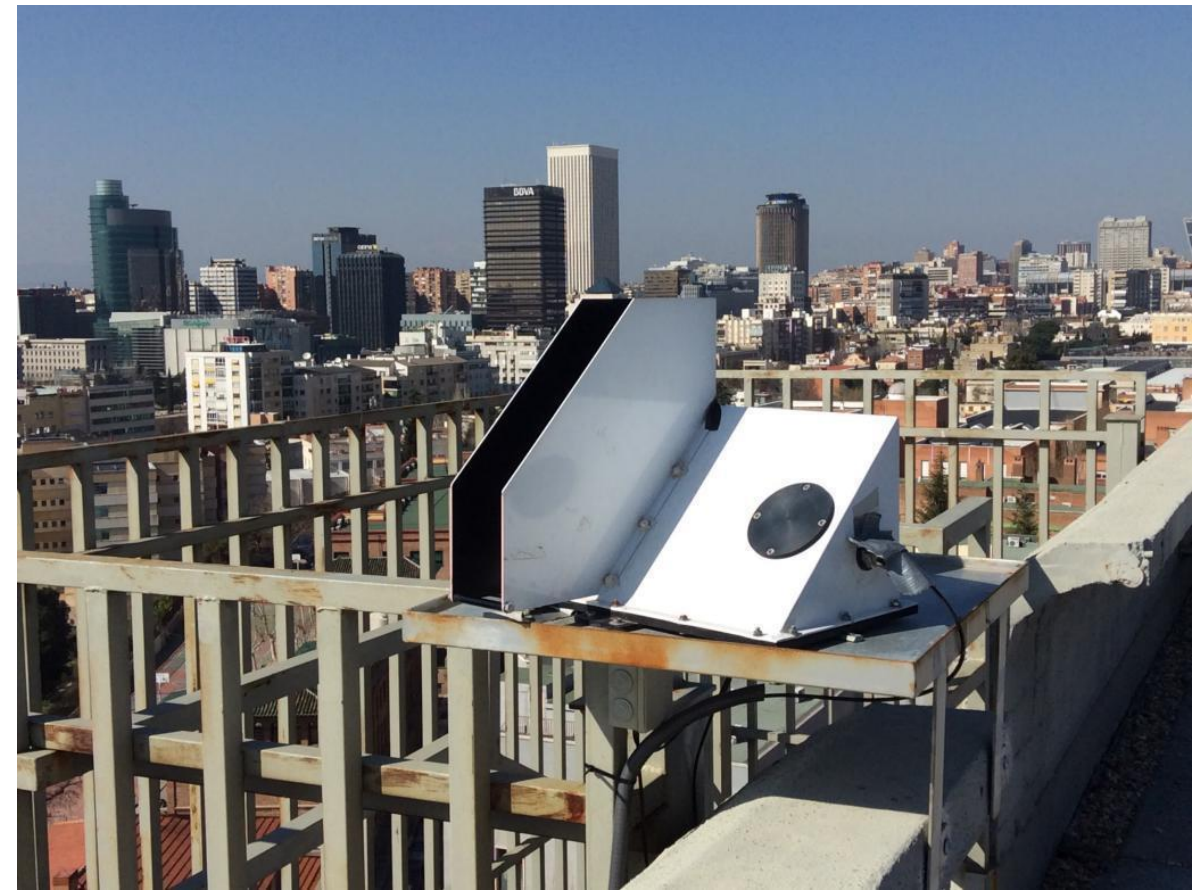

Figure 5.2. MAXDOAS-1D on the roof of the ICA building. 


\subsubsection{Instrument control software}

The spectrometer control and logging software used in this work was developed in Labview by Paul Johnston at the National Institute of Water and Atmospheric Research (NIWA) in Lauder, New Zealand. The program controls the signal saturation, sample and integration times, the stepper motor for the light collector VEAs, displays the spectra and saves them to a daily data file after each integration time.

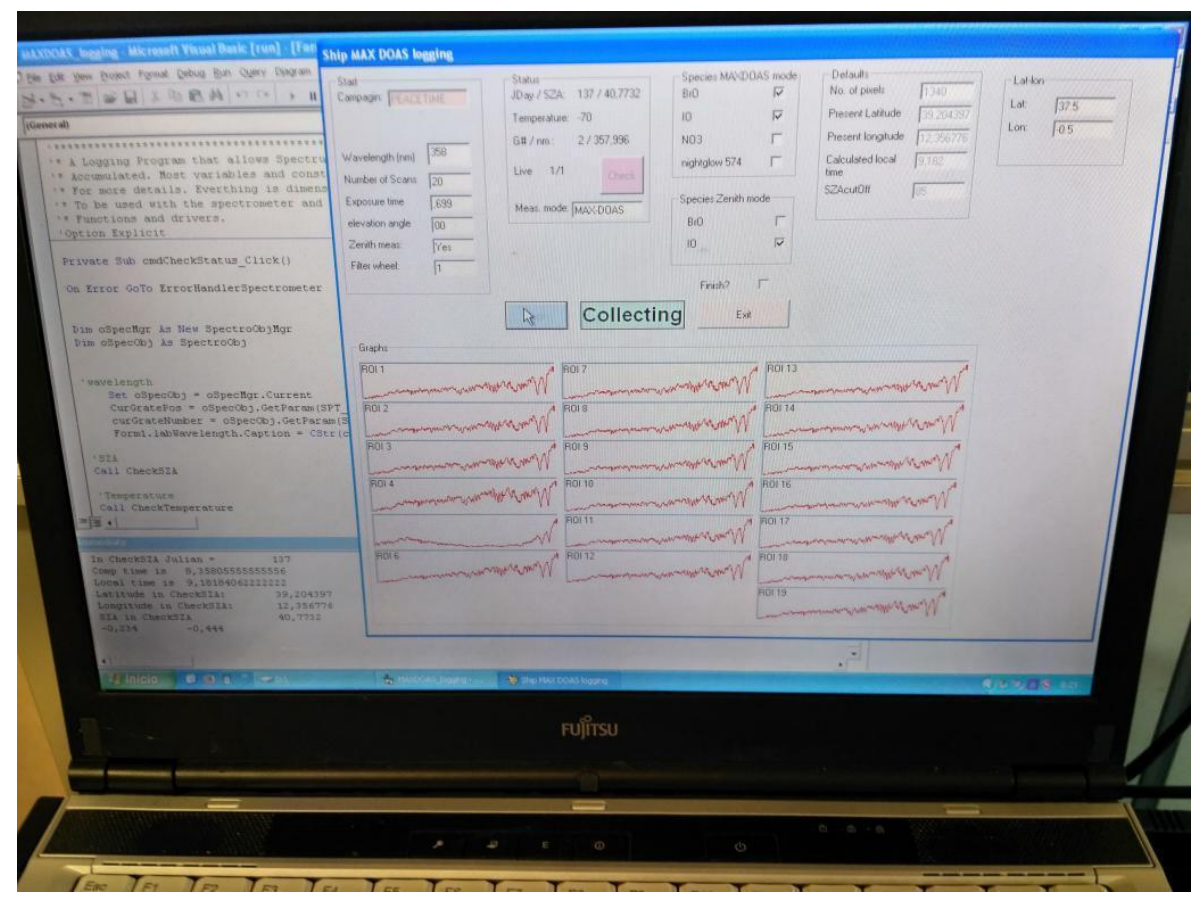

Figure 5.3. MAXDOAS-1D measurements software. The interface includes the spectra collected by each optical fiber, along with all the parameters.

\subsection{Development of a new MAXDOAS-2D instrument}

During the course of this thesis, a new MAXDOAS-2D instrument (Figure 5.5) was built by the Atmospheric Chemistry and Climate group at the Institute of Physical Chemistry Rocasolano (IQFR, CSIC). Its main elements are based on our previous MAXDOAS-1D instrument: a light collector attached to a stepper motor, along with a focusing lens (80 $\mathrm{mm}$ focal length) are responsible for collecting the scattered sunlight. An Ocean Optics, SMA 905 optical fiber of 1-meter length conducts the light through an Ocean Optics, 
HR4000 spectrometer (which incorporates a linear silicon CCD array as detector). The spectrometer wavelength window ranges roughly from $300 \mathrm{~nm}$ to $500 \mathrm{~nm}$ and offers an estimated spectral resolution (FWHM) of about $0.5 \mathrm{~nm}$. An additional stepper motor was included to make the azimuthal movement possible. The instrument incorporates all its components in an outdoor unit. Therefore, to maintain the spectrometer temperature as steady as possible -for both mechanical and wavelength calibration purposes- a Peltier cell was included. Additionally, an Uninterruptible Power Supply (UPS) device provides the necessary power supply and eliminates possible strong power peaks. Two webcams take pictures of -respectively- the cloud cover at each VAA, and monitor the instrument itself. The instrument is quite autonomous and it runs on a homemade Java software. This software controls the movement, the spectra collection and recording, the surrounding accessories and automatically keeps it continuously measuring as long as the Sun is over the horizon.

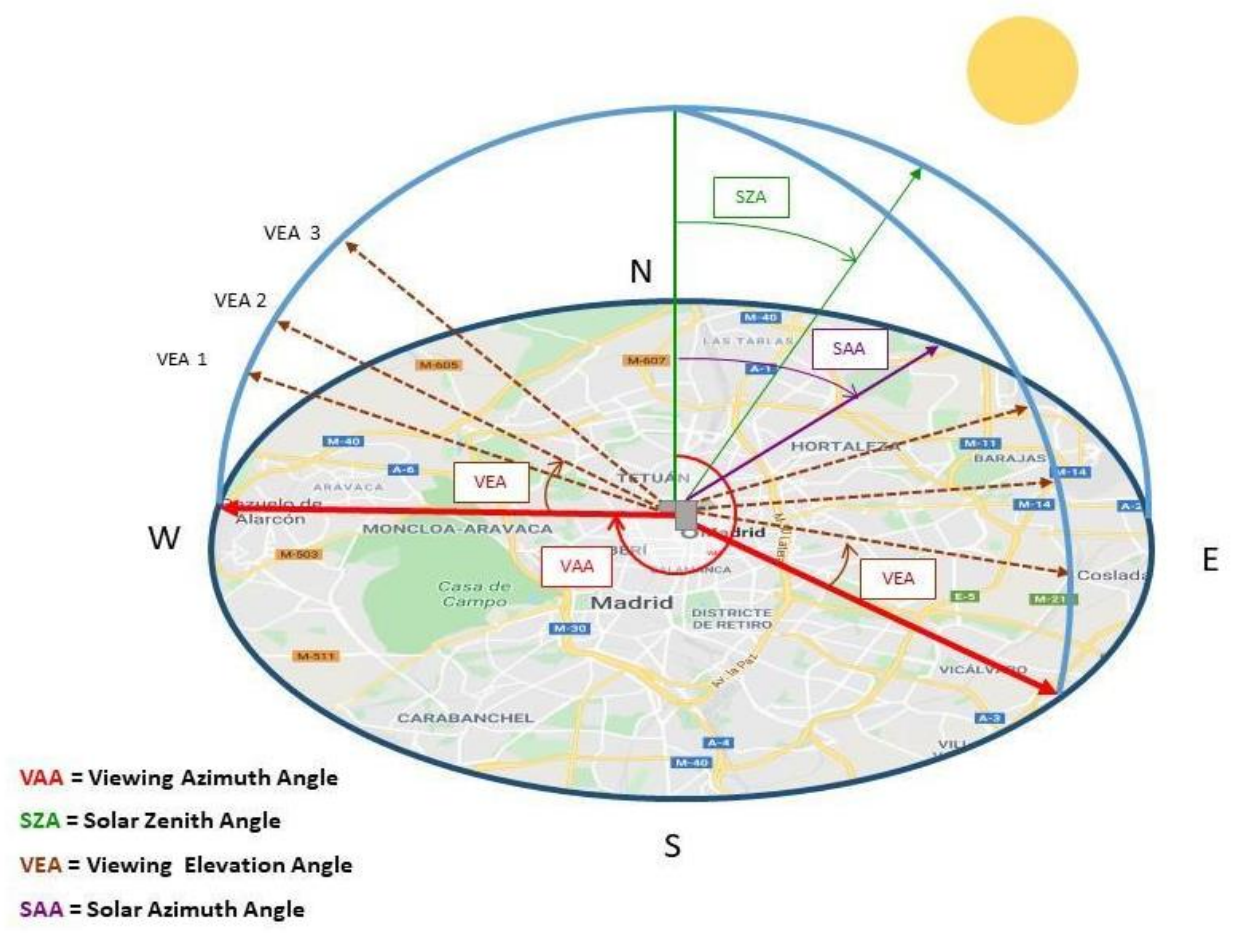

Figure 5.4. MAXDOAS-2D geometry diagram, the background of this picture represents the Madrid city center taken from (C) Google Maps. 
Due to some obstacles that blocked a clear view in some of the VAAs, a small aluminum tower was built to overcome the viewing obstructions and the MAXDOAS-2D instrument was fixed on top of it (see Figure 5.5).
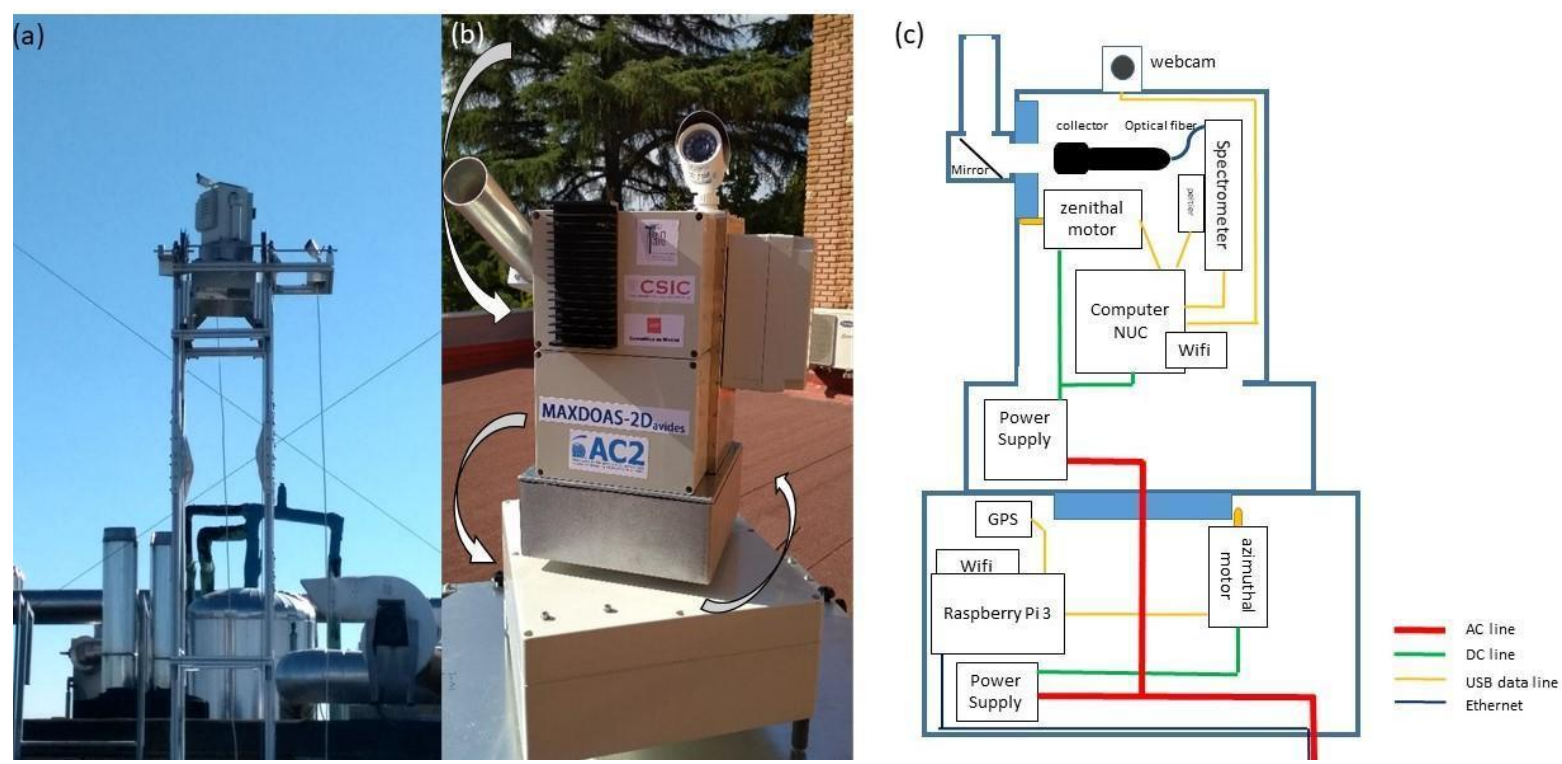

Figure 5.5. a) Aluminum tower with the instrument installed on top of it; b) MAXDOAS-2D instrument; c) MAXDOAS-2D scheme.

\subsubsection{Measurements setup}

In order to sample and analyze a representative portion of the atmosphere over Madrid, selected angular directions were chosen. Starting at a VAA of $0^{\circ}$ (pointing to the north), the MAXDOAS-2D rotated clockwise using steps of $20^{\circ}$ in azimuth. In each azimuthal direction, the ensuing VEA vector was used: 1, 2, 3, 5, 10, 30 and 90 degrees. Therefore, an entire azimuthal lap was completed when the light collector was back again at VAA of $0^{\circ}$.

For every measured spectrum, the spectrometer was able to correct for both electronic offset and dark current effects. Other important parameters for the measurements such as the integration time and the number of scans taken in each angular direction were automatically calculated by the software. More specifically, for this study we set the goal of completing an azimuthal lap in approximately one hour, mainly for an 
easier interpretation of the results and for the subsequent comparison with the in situ instruments of the air quality monitoring network of Madrid. Hence, we chose 24 seconds as the maximum exposure time in each angular combination.

\subsubsection{Alignment procedure}

Once the instrument was set up, we aligned it for both angular movements azimuthal and zenithal- with respect to the geographical North and the local horizontal (i.e. perpendicular to the gravitational plumb), respectively. This process was achieved in two steps: at the beginning, the light collector was coarsely oriented using levels and a compass. Later on, the alignment was refined doing a vertical scan of the Sun (which has a very wellknown position vector) and its angular surroundings at several different times of a clear sky day. The angular differences between the expected theoretical position of the Sun and the measured center of intensity of the registered spectra (a similar approach was done in

Ortega et al., 2015) were estimated and the associated correction applied to the instrument.

Figure 5.6 includes an example of such a solar scan: the point where the red lines cross each other represents the expected angular position of the Sun for that given time, while the yellow sphere stands for the saturation maxima of the collected spectra. The center of intensity in this contour could be assumed as the observed center of the Sun. Finally, both angular differences between the expected and the measured position of the center of the Sun will be accounted for as angular corrections for a more precise alignment. 


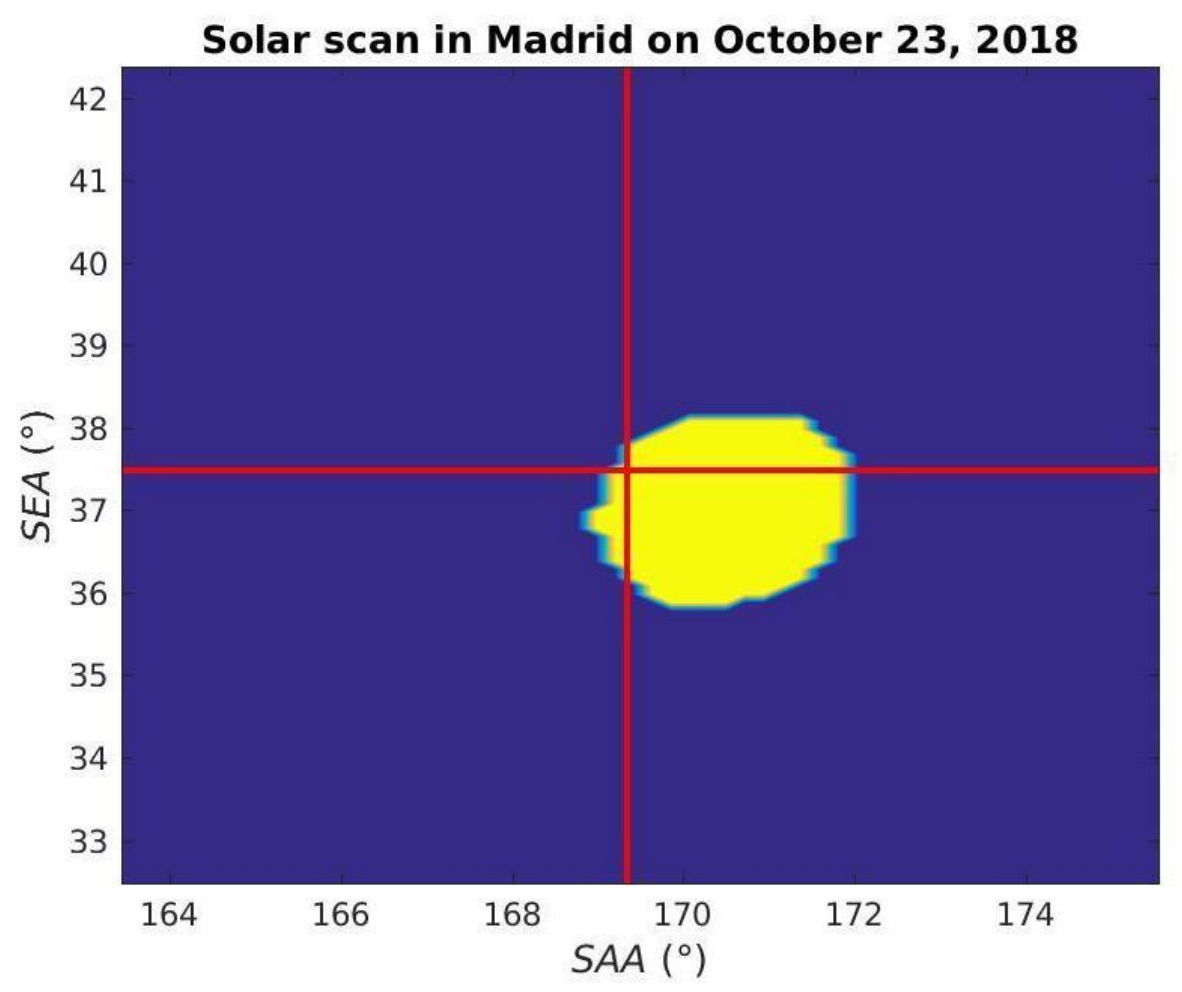

Figure 5.6. Solar scan for the alignment of the MAXDOAS-2D instrument.

\subsection{Common parameters and instrumental effects}

When recording a spectrum, two parameters must be taken into account: the exposure time $\left(t_{\text {exp }}\right)$ and the number of exposures $\left(n_{\text {scans }}\right)$, also called scans. The exposure time is usually given in milliseconds and represents the time during which the detector is exposed to incoming light. The number of scans tells the spectrometer how many exposures it has to add for each measurement. Therefore, the integration time $\left(t_{\text {int }}\right)$ is the total time that the spectrometer has been accumulating spectra: it is equal to the exposure time times the number of exposures $\left(\operatorname{texp}^{*} n_{\text {scans }}\right)$. An optimal combination of these three parameters has to be found in order to maximize the light input as well as the signal-tonoise ratio. 


\subsubsection{Saturation}

In order to maximize the signal-to-noise ratio, it is important to use the dynamic range of the detector. If the chosen exposure time is too short, the recorded spectrum will consist almost exclusively of background noise and the spectral features will not be fully registered, hence yielding large uncertainties. On the other hand, if the exposure time is too long, the detector will be saturated, thus losing spectral features. We can define the saturation level $S L(n)$ of a certain pixel $n$ as:

$$
S L(n)=\frac{c(n) * 100}{n_{\text {scans }} * c_{\max }}
$$

here, $c(n)$ is the number of counts registered in the pixel $n$ and $c_{\max }$ is the maximum number of counts the pixel can register. At saturations above $80-90 \%$, the sensitivity of the CCD detector begins to drop, hence the measured signal is no longer proportional to the incoming photons. To avoid this, a saturation level of about $70-80 \%$ is typically used for atmospheric measurements.

\subsubsection{Electronic offset}

The spectrometer detector receives irradiance (a certain number of photons over each pixel), but in order to treat the data, those irradiances have to be converted to an interpretable signal. The AD-converter takes care of this, producing an analogue voltage (or current) for each pixel: the counts. However, and since the AD-converter cannot transform a negative signal, the CCD detectors include an electronic offset that is added to the measured spectrum (which is photon induced). This shift in the spectral signal must be accounted for in order to avoid non-realistic optical densities. The electronic offset can be estimated by recording an exposure at a very low exposure time with no light entering the spectrometer -for instance blocking the optical fiber- since the measured spectrum will then consist of a combination between the electronic offset and the dark current signals. To minimize the noise, it is appropriate to collect several scans in dark conditions, hence improving the statistical analysis. It can be considered that the electronic offset effect does 
not depend on time, only on the number of scans, therefore the offset-corrected irradiance per each pixel $(\operatorname{Ioc}(n))$ can be calculated as follows:

$$
I_{O C}(n)=I(n)-n_{\text {scans }} * O S(n) / N
$$

where, $I(n)$ is the intensity measured at a certain pixel $n, O S(n)$ is the electronic offset signal previously measured in dark conditions, and $N$ is the number of scans applied to estimate the electronic offset signal.

\subsubsection{Dark current}

The dark current is caused by thermally excited electrons which travel through the semiconductor band gap due to Boltzmann statistics. This means that it strongly depends on the temperature of the detector (Widenhorn et al., 2002). Sometimes, the spectrometers include a cooling device in order to maintain the temperature low (usually at $-50^{\circ} \mathrm{C}$ or even lower), hence reducing the dark current effect.

For a given, constant temperature, the dark current signal is proportional to the exposure time. Therefore, to measure the dark current of an instrument an unique scan, with a very long exposure time, is used, so that the electronic offset is only added once (as explained in section 5.4.2). As we are dealing with background corrections, the conditions here have to be the same as in the electronic offset correction: no light should enter the spectrometer. Once the measurement is recorded, it also has to be offset-corrected, as described in Equation 5.3. As a consequence, the remaining signal consists mainly of dark current. Assuming again that the dark current basically depends on the exposure time, the correction of a given spectrum has to be scaled with respect to the exposure time used in the dark current measurement (Equation 5.3).

$$
I_{D C}(n)=I_{O C}(n)-t_{e x p} * \frac{D_{O S}}{t_{D C}}
$$


Here, $I_{O C}(n)$ is the intensity measured at a pixel $n$ and already corrected for electronic offset. $D_{O S}$ is the measured dark current after correcting for the electronic offset. The exposure time used for the dark current measurement is $t_{D C}$, and $I_{D C}(n)$ is the dark current corrected spectrum at pixel $(n)$.

Figure 5.9 shows an example of the estimated electronic offset and dark current corrections for the MAXDOAS-1D in the UV region. These background measurements were taken with 100 scans at 0.064 seconds per scan for the electronic offset characterization, and 1 scan of 60 seconds for the dark current.
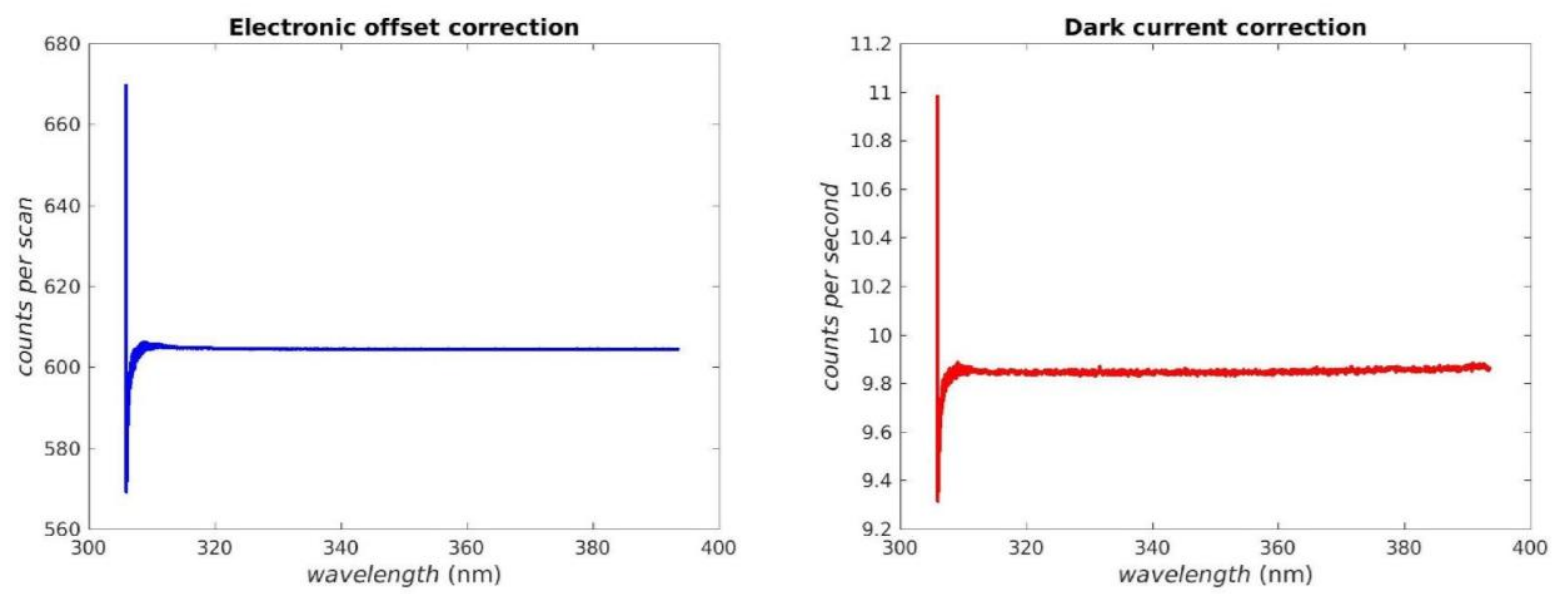

Figure 5.7. Electronic offset and dark current corrections in the UV region for the MAXDOAS-1D instrument.

\subsubsection{Stray light}

The stray light can be defined as the light not desired in the optical design. This stray light can come from either an external source or from the desired source but following nonintended light paths. The main source of the stray light in the DOAS technique consists of reflected light inside the spectrometer other than the diffracted light by the grating, causing an instrumental addition to the measured spectrum. 


\subsection{Wavelength calibration}

The DOAS analysis of atmospheric trace gases requires a careful wavelength calibration, hence minimizing the optical density residuals. Spectrometers collect spectra and assign every pixel with a number of counts that represent the irradiance that reaches each particular pixel. But after that, and in order to evaluate the spectral absorptions, a wavelength has to be associated with each pixel. This step is key for the entire calculations, since not only the tabulated absorption cross sections of the trace gases offer different wavelength grids between them, but there can also be smooth changes in wavelength due to environmental and/or instrumental effects (such as temperature variation). Therefore, a solid, robust wavelength vector is needed to assure a good spectral analysis.

The calibration process has several steps. First, a sound, highly-resolved, extraatmospheric solar spectrum is required. This solar spectrum (measured in vacuum wavelengths) includes the Fraunhofer lines, and is usually measured, with great accuracy, by satellites. For this tabulated solar spectrum, the reference solar atlas based on Chance and Kurucz, 2010, has been used. But this solar atlas has a very high resolution -much higher than the measured spectra- so in order to calibrate the measured spectra with respect to the reference, this solar atlas has to be convolved with the instrument resolution. Usually, a gaussian function -although other fitting functions could be used- of a certain FWHM is applied. Afterwards, a given spectrum can be calibrated -with respect to the solar atlascomparing both wavelength vectors, hence obtaining a wavelength correction. There are several different numerical approaches here, but a general procedure remains: the spectrum that has to be calibrated is compared to the solar atlas, hence both spectra are inputs of a non-linear, least-squares fitting that applies the following parameters: the FWHM of the convolution function, and the wavelength correction between them (usually in the form of a low-order polynomial). The fit is based on the wavelength position of the absorption lines, rather than the actual amplitudes (which would be related to the absorptions themselves, as in the DOAS analysis). In spite of this, often a Ring spectrum is also included in the calibration, in order to cancel out some of the spectral features. Once 
an optimal set of parameters is found, the spectrum is corrected and considered wellcalibrated. Figure 5.8 shows an example of a wavelength calibration of a noon spectrum taken with the MAXDOAS-2D instrument. A very good simulation can be observed, along with the calibration parameters (estimated FWHM and shift).

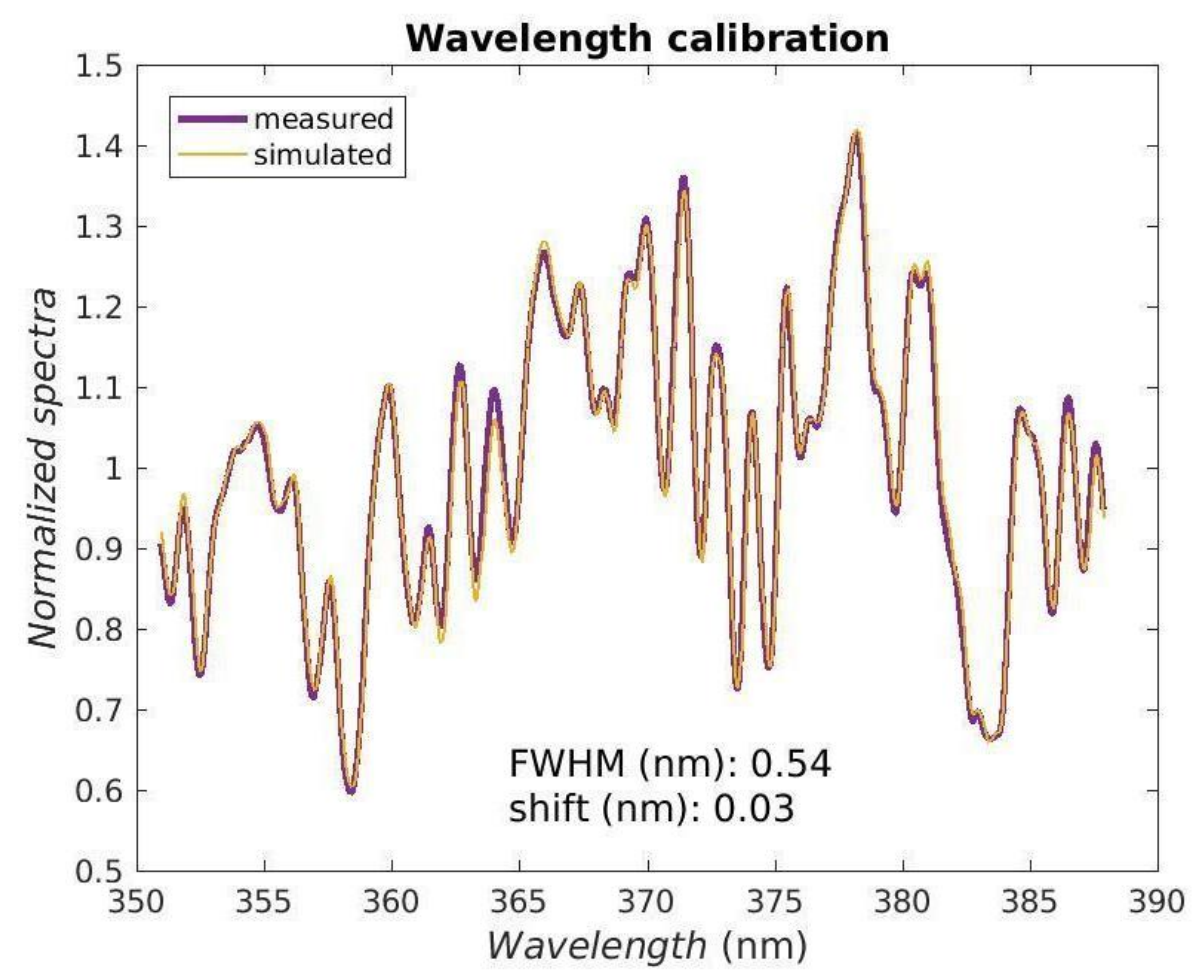

Figure 5.8. Example of the wavelength calibration of a spectrum. It was a zenith spectrum collected with the MAXDOAS-2D in Madrid at around noon on May 11, 2019. The estimated spectral resolution (FWHM) is of $0.54 \mathrm{~nm}$, with an estimated wavelength shift correction of $0.03 \mathrm{~nm}$.

However, as mentioned in Chapter 3, we need two spectra (a low VEA spectrum and the zenith spectrum) in order to get the optical density and analyse the absorptions. But if we calibrate in wavelength for both spectra, there might be slight differences in each final wavelength vector, so a choice has to be made. Typically, the zenith spectrum (i.e. VEA $90^{\circ}$ ) is set as the wavelength reference, since it has less absorptions and covers smaller light paths, thus being more comparable to the solar atlas, thereby yielding an easier calibration. 
Hence the other spectrum (collected at a low VEA) is interpolated to the optimized wavelength vector of the reference spectrum. Finally, all the absorption cross sections included in the analysis are convolved using the optimal FWHM of the fitting and subsequently interpolated to the reference wavelength vector.

On a separate note, the mentioned optimal FWHM is used as an estimation of the spectral resolution of the instrument, since it is representative of how wide you have to degrade the high-resolution solar atlas in order to have spectral features comparable with those measured by the instrument.

Although this wavelength calibration process could be performed over the entire spectrum, usually it is done over a window that is slightly larger than the analysis window. Moreover, within this main calibration window, there could also be several sub-windows over which the non-linear fitting is carried out. 


\section{$6 \mathrm{NO}_{2}$ AND HONO}

In this chapter, measurements of the atmospheric compounds $\mathrm{NO}_{2}$ and $\mathrm{HONO}$ are described. First, the tropospheric chemistry of these compounds is explained; after that, the details of the measured spectra, the DOAS analysis and the inversion parameters applied are also described. Finally, the vertical concentration profiles of $\mathrm{NO}_{2}$ and $\mathrm{HONO}$ and their seasonal evolution are studied. The results have been published in Garcia-Nieto et. al. 2018.

\subsection{Introduction}

In atmospheric chemistry, nitrogen oxides $\left(\mathrm{NO}_{\mathrm{x}}\right)$ are highly reactive in the atmosphere and they have a great impact on its oxidation capacity. Natural sources of $\mathrm{NO}_{\mathrm{x}}$ are biomass burning, lightning, soil or volcanic emissions. However, most of the $\mathrm{NO}_{\mathrm{x}}$ sources in urban environments come from anthropogenic emissions including traffic, industrial activities and transport. This anthropogenic contribution constitutes more than half of the global $\mathrm{NO}_{2}$ emissions.

Atmospheric $\mathrm{NO}_{2}$ can be removed by the following oxidation process:

$$
\mathrm{NO}_{2}+\mathrm{OH}+\mathrm{M} \rightarrow \mathrm{HNO}_{3}+\mathrm{M}
$$

The removal of $\mathrm{HNO}_{3}$ proceeds via wet and dry deposition. The wet deposition is called acid rain. Another important reaction is the one that involves $\mathrm{NO}_{2}$ with ozone.

$$
\mathrm{NO}_{2}+\mathrm{O}_{3} \rightarrow \mathrm{NO}_{3}+\mathrm{O}_{2}
$$


The rate coefficient for this reaction is small, although it cannot be neglected in areas with high ozone concentrations in the absence of sunlight. $\mathrm{NO}_{3}$ is quickly photolyzed during the early morning, but at night it reacts with $\mathrm{NO}_{2}$ and form the night time reservoir species:

$$
\begin{aligned}
& \mathrm{NO}_{3}+\mathrm{NO}_{2}+\mathrm{M} \rightarrow \mathrm{N}_{2} \mathrm{O}_{5}+\mathrm{M} \\
& \mathrm{N}_{2} \mathrm{O}_{5}+\mathrm{M} \rightarrow \mathrm{NO}_{3}+\mathrm{NO}_{2}+\mathrm{M}
\end{aligned}
$$

When the Sun rises again, the $\mathrm{N}_{2} \mathrm{O}_{5}$ can form $\mathrm{NO}_{3}$ or $\mathrm{NO}_{2}$ following these reactions:

$$
\mathrm{N}_{2} \mathrm{O}_{5}+\mathrm{hv} \rightarrow \mathrm{NO}_{2}+\mathrm{NO}+\mathrm{O}_{2}
$$

At sunlit hours, the $\mathrm{NO}_{2}$ oxidation reactions that occur in the atmosphere are the following (Leighton, 1961).

$$
\begin{aligned}
& \mathrm{O}\left({ }^{3} \mathrm{P}\right)+\mathrm{O}_{2}+\mathrm{M} \rightarrow \mathrm{O}_{3} \\
& \mathrm{NO}+\mathrm{O}_{3} \rightarrow \mathrm{NO}_{2}+\mathrm{O}_{2} \\
& \mathrm{NO}_{2}+\mathrm{hv} \rightarrow \mathrm{NO}+\mathrm{O}\left({ }^{3} \mathrm{P}\right)(\lambda<420 \mathrm{~nm})
\end{aligned}
$$

Nitrous acid stands as one of the main species in tropospheric chemistry, primarily in polluted, urban regions. Since several decades ago, it is known that HONO plays a key role in the chemistry of the troposphere because, through its fast photodissociation, it is considered as one of the main sources of the hydroxyl radical, the most relevant oxidant in the atmosphere and responsible for the degradation of most atmospheric pollutants (Perner and Platt, 1979; Heard and Pilling, 2003). This effect takes place in the sunlit hours in polluted regions (Zhang et al., 2016), and thus has associated impacts on the formation of secondary oxidation products such as $\mathrm{O}_{3}$ and Secondary Organic Aerosols (SOA) 
(Volkamer et al., 2007). Therefore, the evaluation of HONO vertical concentration profiles and their temporal evolution is important for urban atmospheric chemistry.

Several HONO production pathways have been proposed: for instance, the heterogeneous conversion of $\mathrm{NO}_{2}$ on humid organic surfaces or on inorganic ground surfaces (like soil, buildings, vegetation and aerosols). These processes are thought to be a major HONO source, and very likely the main mechanism for its build-up during the night (Wojtal et al., 2011):

$$
2 \mathrm{NO}_{2}+\mathrm{H}_{2} \mathrm{O}+\text { surface }-->\mathrm{HONO}+\mathrm{HNO}_{3}
$$

Direct emissions from vehicles and combustion processes have also been identified as sources of $\mathrm{HONO}$, with a molar emission ratio of $\mathrm{HONO}$ to nitrogen oxides $\left(\mathrm{NO}_{x}=\mathrm{NO}+\right.$ $\mathrm{NO}_{2}$ ) ranging from $10^{-4}$ to $10^{-2}$ (Kurtenbach et al., 2001):

$$
\mathrm{NO}+\mathrm{OH}+\mathrm{M}-->\mathrm{HONO}+\mathrm{M}
$$

Reaction (R6.10) works only during daytime, when the $\mathrm{OH}$ and NO concentrations are high. The loss processes of HONO include dry deposition during night-time and the reaction between $\mathrm{HONO}$ and $\mathrm{OH}$ :

$$
\mathrm{HONO}+\mathrm{OH}-->\mathrm{H}_{2} \mathrm{O}+\mathrm{NO}_{2}
$$

However, reaction (R6.11) is comparatively very slow, and the mentioned sink reactions are negligible compared to the prominent, fast photolytic pathway, which occurs at a yearly averaged rate of the order of $10^{-3} \mathrm{~s}^{-1}$ around noon for mid-latitudes (Hendrick et al., 2014):

$$
\mathrm{HONO}+\mathrm{hv}(\lambda<400 \mathrm{~nm})-->\mathrm{OH}+\mathrm{NO}
$$


Numerous studies have shown that this rapid photolysis (of accumulated HONO during the night) can actually be the dominant early morning source of $\mathrm{OH}$ (Hendrick et al., 2014), yielding similar $\mathrm{OH}$ production rates as the $\mathrm{O}_{3}$ photolysis, which is regarded as the major $\mathrm{OH}$ source in remote atmospheres (Alicke et al., 2003).

Nevertheless, although several field campaigns and experiments have been carried out during the past decades, the main HONO source mechanisms are not fully characterised yet.

Given its relevance in atmospheric chemistry, knowledge of the HONO concentration and spatial distribution is key for our understanding of the oxidation capacity in urban atmospheres (Acker et al., 2006). Most HONO observations in urban environments use in situ techniques or long path averaged DOAS observations (Heland et al., 2001), which are unable to provide information about the vertical distribution of the molecule within the planetary boundary layer, though there are also some previous HONO measurements using the MAXDOAS technique (Wang et al., 2017).

\subsection{Experimental}

For the measurements, the MAXDOAS-1D placed on the roof of the ICA building (inside the CSIC campus in Madrid, see more details in Section 5.2) has been used.

For this chapter, one year of ground-based MAXDOAS observations of $\mathrm{NO}_{2}$, $\mathrm{HONO}$ and aerosol extinction profiles in Madrid is presented; the measurements range from January 1, 2016 to December 31, 2016 and represent the first HONO time series measurements performed in Madrid. Vertical profiles, as well as their diurnal and seasonal evolution during 2016 are derived. Tables 6.1 and 6.2 summarize the DOAS fit settings used for the retrieval of $\mathrm{NO}_{2}, \mathrm{O}_{4}$ and $\mathrm{HONO}$. 
Table 6.1. DOAS fit settings for the retrieval of $\mathrm{O}_{4}$ and $\mathrm{NO}_{2}$ in the UV range.

\begin{tabular}{|c|c|}
\hline Parameter & Value \\
\hline Fitting window & $338-370 \mathrm{~nm}$ \\
\hline Wavelength calibration & $\begin{array}{l}\text { Based on reference solar atlas (Chance and } \\
\text { Kurucz, 2010). }\end{array}$ \\
\hline Zenith reference & Scan \\
\hline Polynomial order & 5 \\
\hline Intensity offset & Order 1 \\
\hline Shift & $\begin{array}{l}\text { The measured spectra and the Ring cross } \\
\text { sections were allowed to shift and stretch } \\
\text { (order } 1 \text { ) in wavelength. }\end{array}$ \\
\hline Molecule & Cross section \\
\hline $\mathrm{O}_{4}$ & $293 \mathrm{~K}$ (Thalman and Volkamer, 2013) \\
\hline $\mathrm{NO}_{2 a}$ & 298 K (Vandaele et al., 1998) \\
\hline $\mathrm{NO}_{2 b}$ & $220 \mathrm{~K}$ (Vandaele et al., 1998) \\
\hline $\mathrm{O}_{3 а}$ & $223 \mathrm{~K}$ (Serdyuchenko et al., 2014) \\
\hline $\mathrm{O}_{3 b}$ & $243 \mathrm{~K}$ (Serdyuchenko et al., 2014) \\
\hline $\mathrm{HCHO}$ & $297 \mathrm{~K}$ (Meller and Moortgat, 2000) \\
\hline $\mathrm{BrO}$ & $223 \mathrm{~K}$ (Fleischmann et al., 2004) \\
\hline Ring & Calculated by QDOAS \\
\hline
\end{tabular}


Table 6.2. DOAS fit settings for the retrieval of HONO in the UV range.

\begin{tabular}{|c|c|}
\hline Parameter & Value \\
\hline Fitting window & $337-375 \mathrm{~nm}$ \\
\hline Wavelength calibration & $\begin{array}{l}\text { Based on reference solar atlas (Chance and } \\
\text { Kurucz, 2010). }\end{array}$ \\
\hline Zenith reference & Scan \\
\hline Polynomial order & 5 \\
\hline Intensity offset & - \\
\hline Shift & $\begin{array}{l}\text { The measured spectra and the Ring cross } \\
\text { sections were allowed to shift and stretch } \\
\text { (order } 1 \text { ) in wavelength. }\end{array}$ \\
\hline Molecule & Cross section \\
\hline HONO & 296 K (Stutz et al., 2000) \\
\hline $\mathrm{O}_{4}$ & $293 \mathrm{~K}$ (Thalman and Volkamer, 2013) \\
\hline $\mathrm{NO}_{2} \mathrm{a}$ & 298 K (Vandaele et al., 1998) \\
\hline $\mathrm{NO}_{2} \mathrm{~b}$ & $220 \mathrm{~K}$ (Vandaele et al., 1998) \\
\hline $\mathrm{O}_{3} \mathrm{a}$ & $223 \mathrm{~K}$ (Serdyuchenko et al., 2014) \\
\hline $\mathrm{O}_{3} \mathrm{~b}$ & $243 \mathrm{~K}$ (Serdyuchenko et al., 2014) \\
\hline $\mathrm{BrO}$ & $223 \mathrm{~K}$ (Fleischmann et al., 2004) \\
\hline Ring & Calculated by QDOAS \\
\hline
\end{tabular}




\subsection{Analysis methods}

\subsubsection{Cloud screening and quality filters}

After crossing the cloud-screened AERONET database (explained in Subsection 4.5) with our own spectra database and applying the quality filters explained in Subsection 4.7, 269 days remained, which are the days included in this section. Regarding their distribution among seasons, we have 58 days for winter (defined here as from January to March, both included), 69 days for spring (April to June), 85 days for summer (July to September) and 57 days for autumn (October to December).

\subsubsection{Spectral detection}

As explained before, the DOAS technique adjusts the measured differential optical density to the various differential absorption cross sections included in the analysis. This produces the so-called spectral fittings (Figures 6.1 for $\mathrm{O}_{4}$ and $\mathrm{NO}_{2}$ and 6.2 for $\mathrm{HONO}$ ).
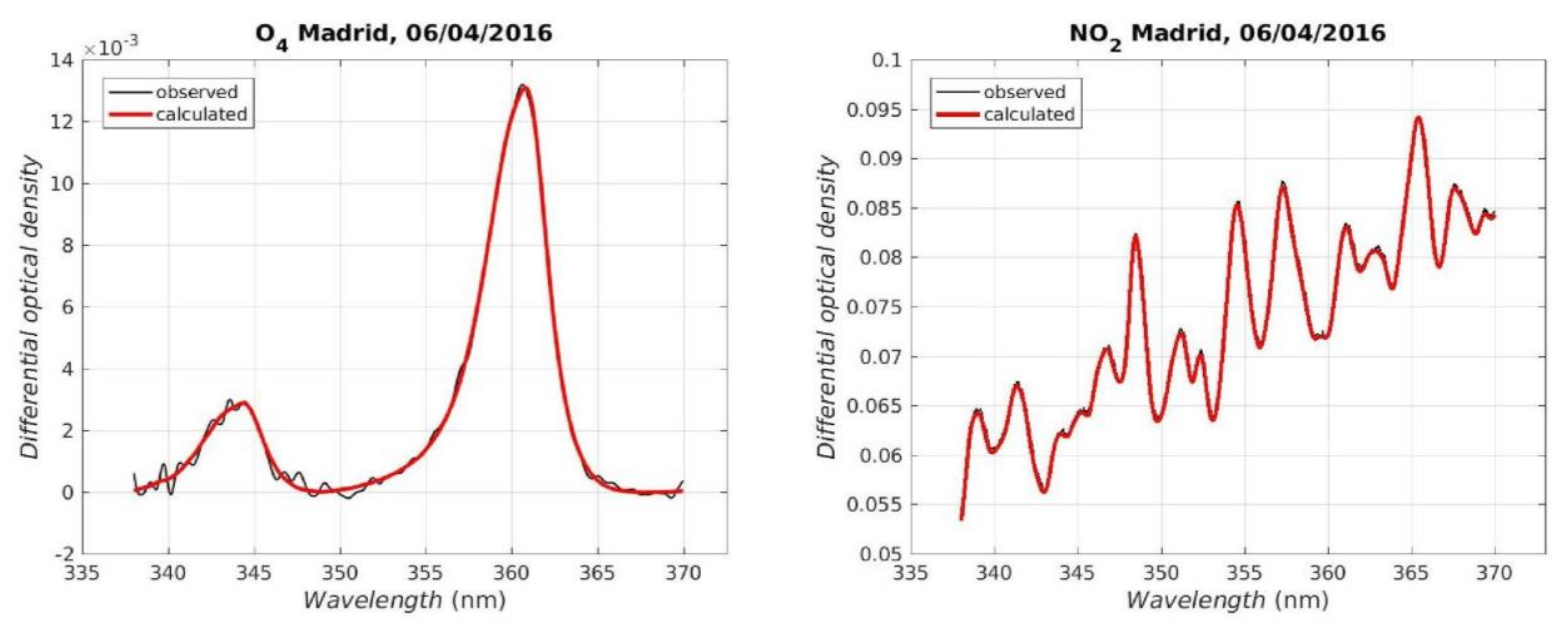

Figure 6.1. Examples of $\mathrm{O}_{4}$ and $\mathrm{NO}_{2}$ spectral fits in the UV region on April 6, 2016. 


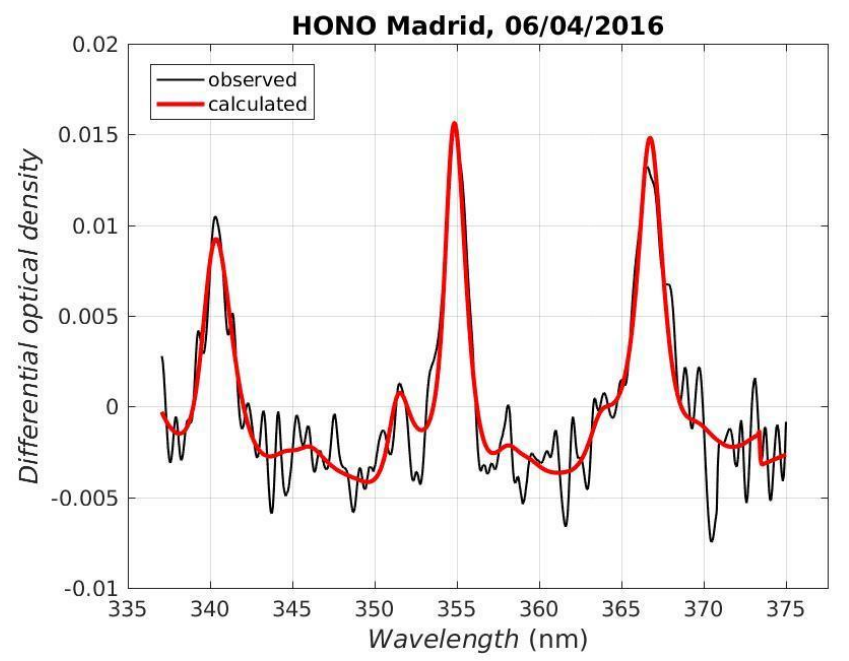

Figure 6.2. Example of a HONO spectral fit in the UV region on April 6, 2016.

\subsubsection{Inversion parameters}

Since atmospheric chemistry models have been reported to systematically underestimate HONO concentration values with respect to the measured profiles (Lee et al., 2016), the geometric approximation explained in Section 3.4 has been chosen. We assumed $0.5 \mathrm{~km}$ as scaling height constant for all the a priori profiles and a fixed total vertical amount: for the aerosol retrieval we took the AOD from AERONET while for $\mathrm{NO}_{2}$ and HONO we applied the geometrical approximation to the measured DSCDs at EVA $20^{\circ}$.

\subsection{Results and discussion}

\subsection{1 $\mathrm{O}_{4}$ DSCDs correction factor}

There are several different $\mathrm{O}_{4}$ cross sections reported in the literature, each of them slightly different from the others: for instance, the peak of the $\mathrm{O}_{4}$ absorption band around $360 \mathrm{~nm}$ is $5.4 \times 10^{-46} \mathrm{~cm}^{5}$ molecule ${ }^{-2}$ in Wagner et al., 2002, while in the one used in this study (Table 6.1) the maximum value at the same wavelength is $4.28 \times 10^{-46} \mathrm{~cm}^{5}$ molecule ${ }^{-2}$. These differences are not large, but since both the shape and the absolute values of the cross sections have an influence in the DOAS analysis, it is worth to take this effect into account. 
In order to do that we compared, for a clear-sky and low aerosol loading day, the measured $\mathrm{O}_{4}$ DSCDs with the simulated DSCDs using the radiative transfer model bePRO. Under the aforementioned atmospheric conditions, the $\mathrm{O}_{4}$ DSCDs at high VEAs (higher than $10^{\circ}-15^{\circ}$ ) are rather insensitive to changes in the pressure, temperature or aerosol optical properties (Wagner et al., 2009).

Figure 6.3 shows this effect. For VEAs $15^{\circ}, 20^{\circ}$ and $30^{\circ}$ some different atmospheric conditions were chosen: in particular, we assumed an exponentially decreasing profile for the aerosol load with a certain scaling height and a given total AOD (ranging between 0.25 and $0.75 \mathrm{~km}$ the former and from 0.025 to 0.05 the latter, see Figure 6.3). It can be observed that there is a good agreement for each set. Therefore, the uncertainty related to the $\mathrm{O}_{4}$ cross sections -along with their dependencies on pressure and temperature, which are not well known (Wagner et al., 2002)- can be accounted for the differences between the measured and simulated $\mathrm{O}_{4}$ DSCDs.

Comparing the measured and simulated $\mathrm{O}_{4}$ DSCDs for the selected clear day January 16 , 2016- we found that the measurements were consistently about $25 \%$ higher than the simulations (Figure 6.3, similar correction factors were reported by other authors (Wagner et al., 2009; Clémer et al., 2010)). Therefore, to take this systematic difference into account we introduced the following factor for the subsequent $\mathrm{O}_{4}$ calculations:

$$
\mathrm{O}_{4} \text { DSCD corrected }=\mathrm{O}_{4} \text { DSCD measured } * 0.8
$$



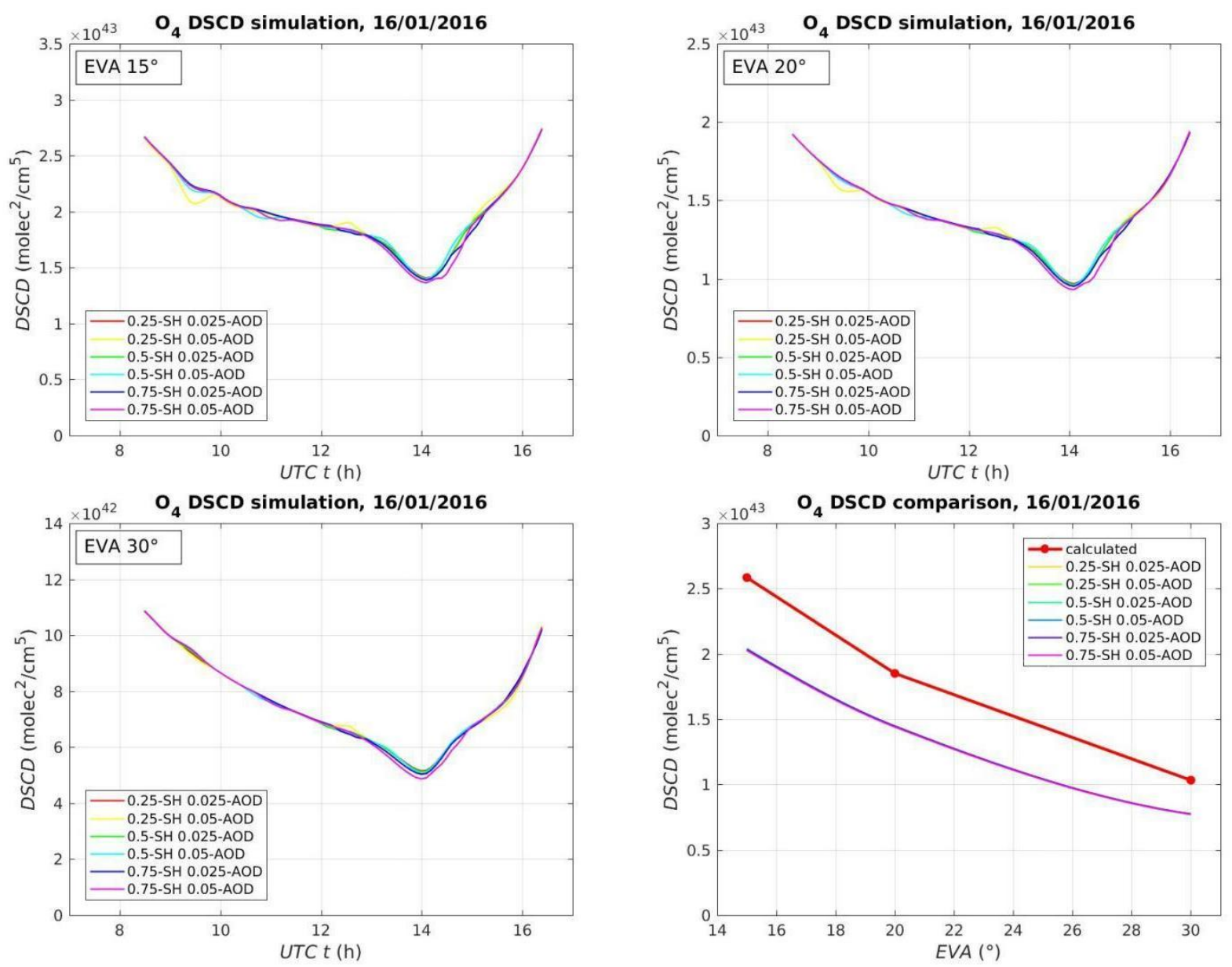

Figure 6.3. Comparison between simulated and measured $\mathrm{O}_{4}$ DSCDs on January 16, 2016 (clear sky day).

\subsubsection{Aerosols extinction and vertical profiles}

Once the $\mathrm{O}_{4} \mathrm{DSCDs}$ factor is estimated, the resulting $\mathrm{O}_{4} \mathrm{DSCD}$ are introduced as a primary input in the profile retrieval as explained in Chapter 4. The comparison -and correlation- between the simulated and measured DSCDs is representative of the goodness of the adjustment. Figure 6.4 shows an example of such a comparison, performed for the same day chosen for the $\mathrm{O}_{4}$ DSCDs correction factor estimation. 

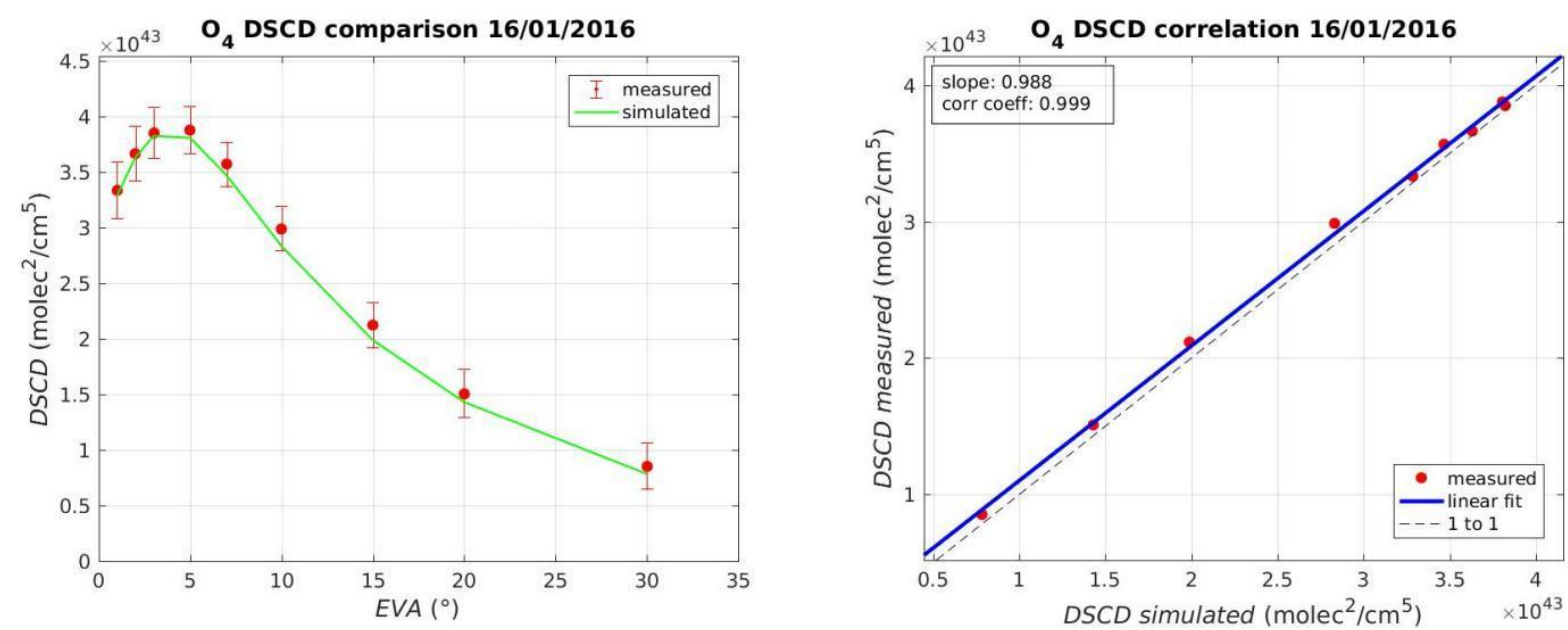

Figure 6.4. Comparison and correlation between measured and simulated $\mathrm{O}_{4} \mathrm{DSCDs}$ on January 16, 2016.

The optimal set of simulated $\mathrm{O}_{4}$ DSCDs come from the aerosol extinction vertical profile that generates it. Figure 6.5 shows an example of aerosol extinction vertical profile for the mentioned day January 16, 2016.

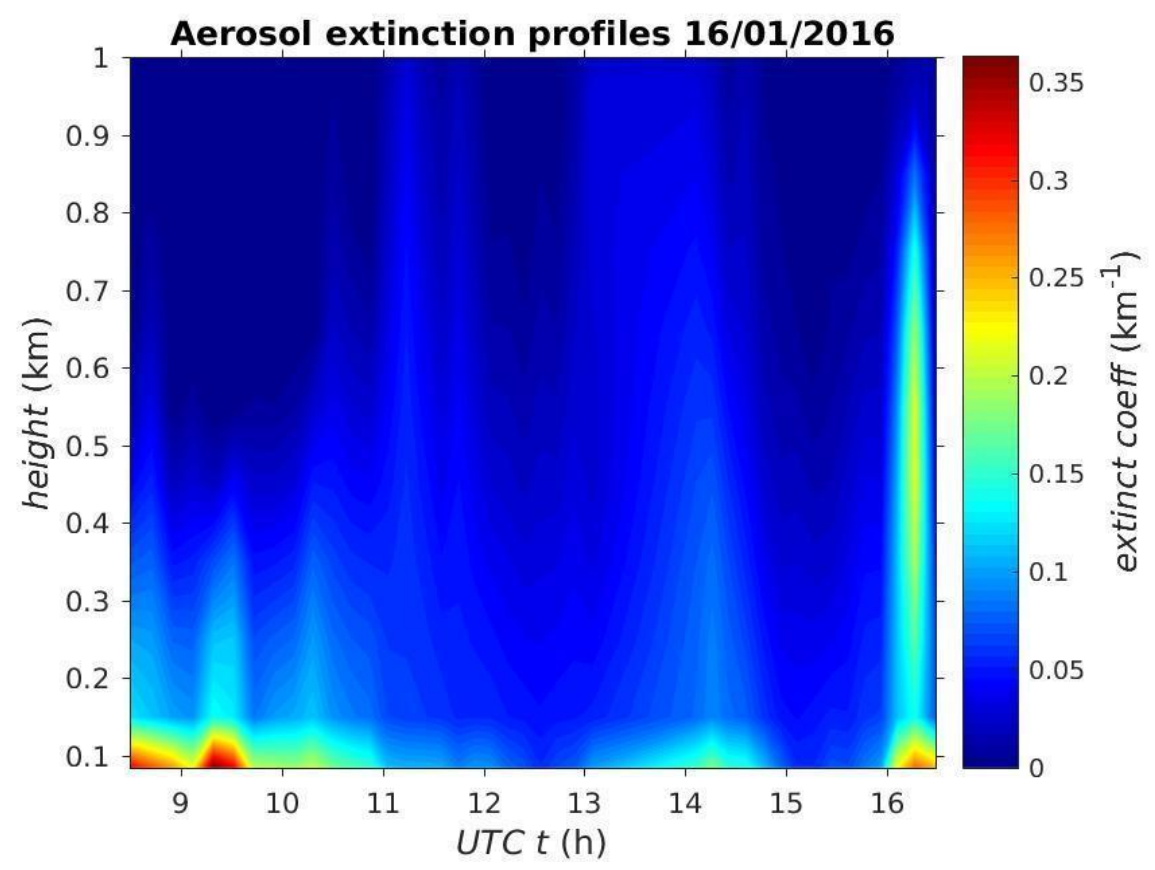

Figure 6.5. Vertical profiles of aerosol extinction coefficients for the UV region in Madrid on January 16, 2016. 
Once the influence of the extinction by aerosols is subtracted, an analogous process is done in order to get the $\mathrm{NO}_{2}$ and $\mathrm{HONO}$ vertical profiles. Figure 6.6 includes the comparison and correlation between measured and simulated $\mathrm{NO}_{2}$ DSCDs on January 16 , 2016, while Figure 6.7 includes the associated $\mathrm{NO}_{2}$ vertical concentration profiles during the same day.
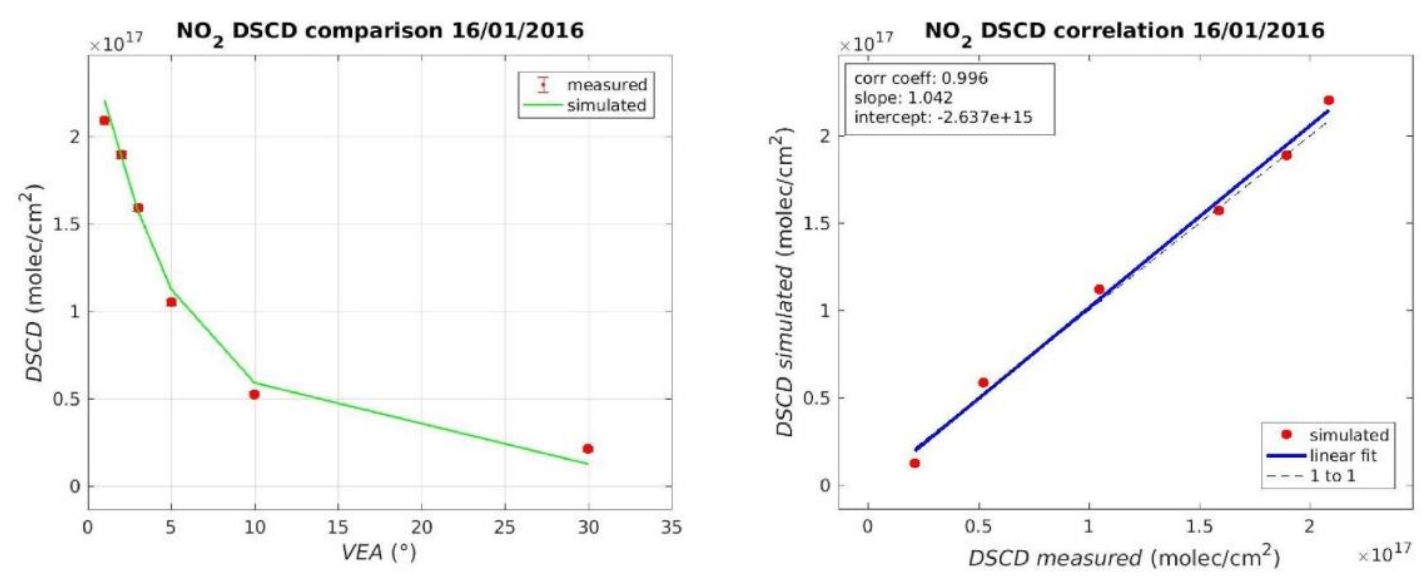

Figure 6.6. Comparison and correlation between measured and simulated $\mathrm{NO}_{2} \mathrm{DSCD}$ on January 16, 2016.

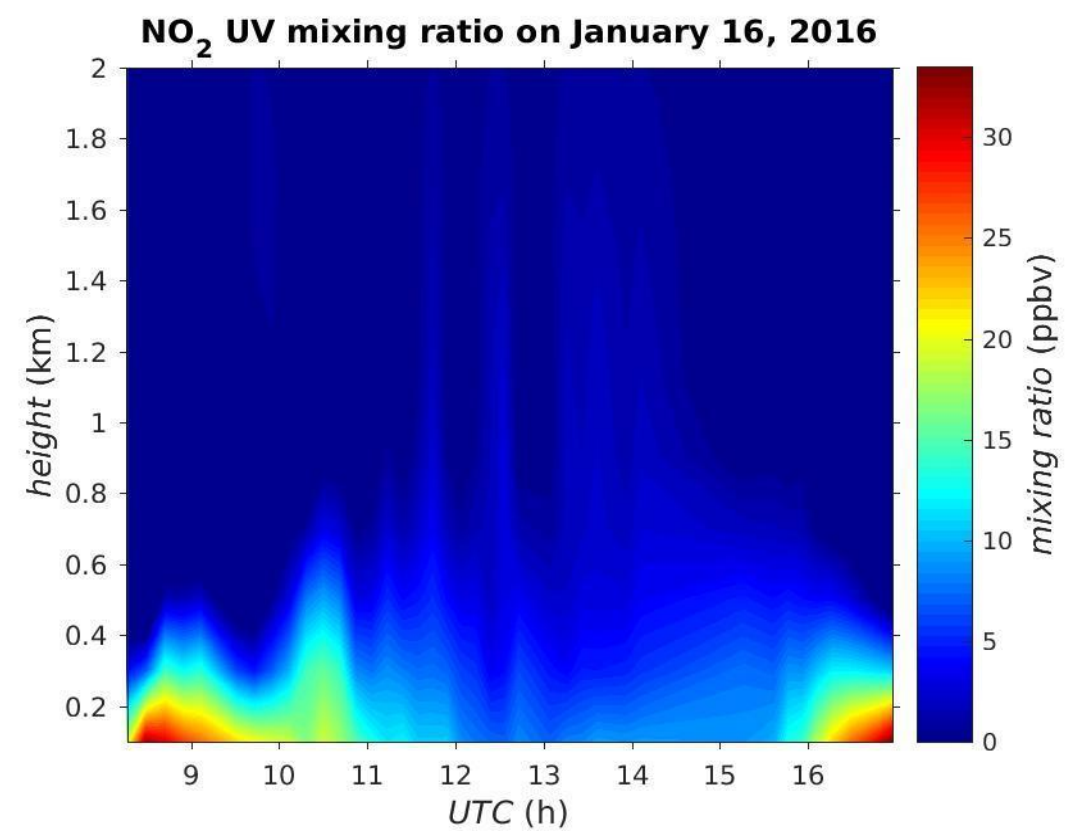

Figure 6.7. Vertical profiles of $\mathrm{NO}_{2}$ for the UV region in Madrid on January 16, 2016. 
Figure 6.7 shows maximum $\mathrm{NO}_{2}$ mixing ratio values of about $30 \mathrm{ppbv}$ in the early morning and in the evening. Both peaks can be related to traffic rush hours in the city.

As an example for HONO, Figure 6.8 contains the comparison and correlation between measured and simulated HONO DSCDs on August 1, 2016, while Figure 6.9 includes the associated HONO vertical concentration profiles during the same day.
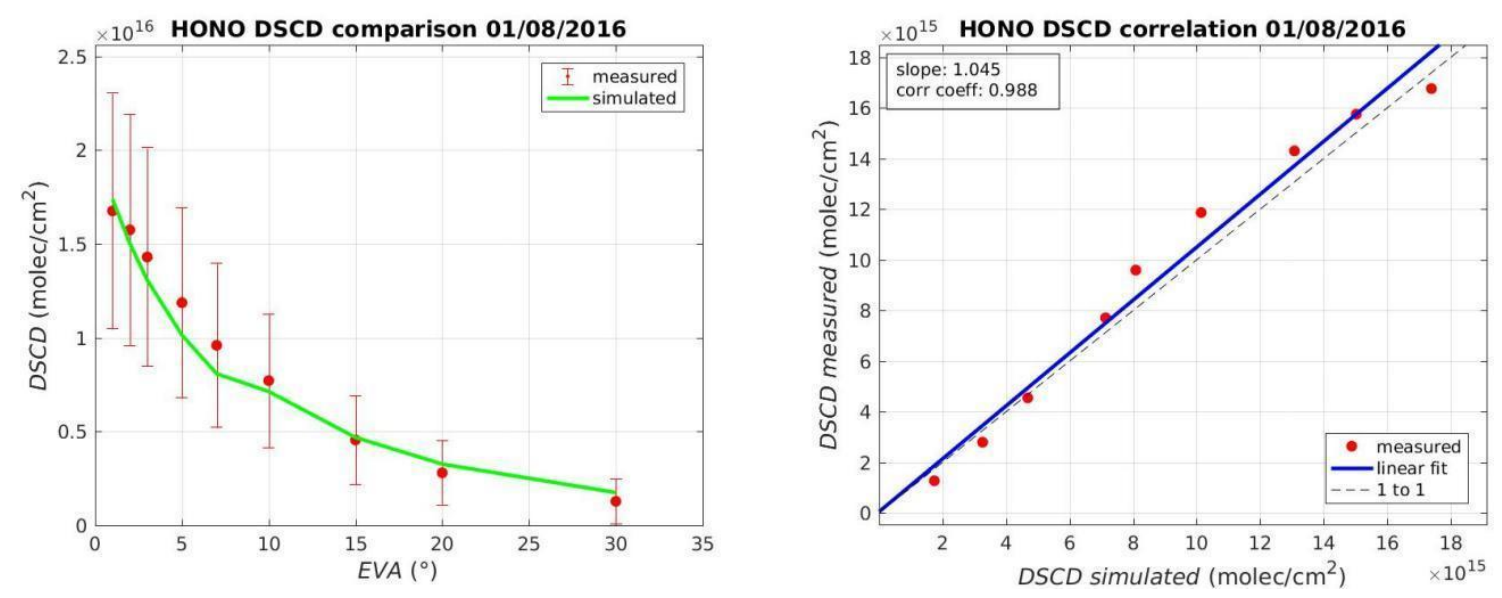

Figure 6.8. Comparison and correlation between measured and simulated HONO DSCDs on January 16, 2016.

It can be observed in Figure 6.9 that the profile exhibits the expected HONO concentration swift decay -by photolysis- as soon as the Sun rises in the early morning as well as the beginning of its accumulation in the late evening when photolysis is much less efficient or absent. Maximum values between 2 and $2.5 \mathrm{ppbv}$ are reached at the beginning ( 6 UTC) and the end (19 UTC) of the sunlight hours and are located within the first $500 \mathrm{~m}$; while minimum values lower than 1 ppbv are found around noon, spanning up to $150 \mathrm{~m}$ height, with almost null HONO background for higher altitudes at that time. The average temporal decay rate as the solar elevation angle increases in the morning is close to -160 pptv $\mathrm{h}^{-1}$ for the first $100 \mathrm{~m}$ height layer, whereas concerning the altitude, HONO concentration falls below $10 \%$ of the ground level value for altitudes higher than $1 \mathrm{~km}$. 


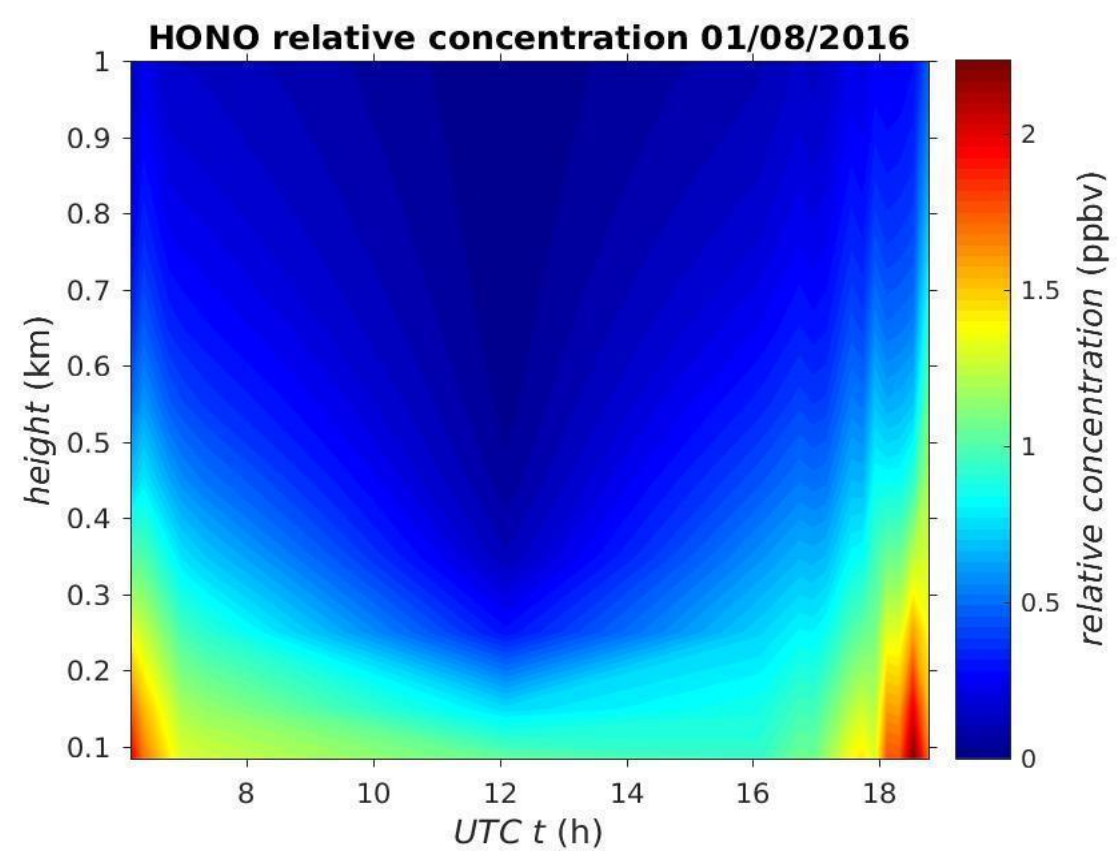

Figure 6.9. HONO vertical concentration profiles in Madrid on January 16, 2016.

\subsubsection{Seasonality}

In this section we report the seasonal variability of $\mathrm{NO}_{2}$ and $\mathrm{HONO}$ levels in Madrid. To illustrate the behaviour of the profiling tool in relation to the measurements, Figure 6.10 presents the long-term correlation of measured and simulated DSCDs both for aerosols and $\mathrm{NO}_{2}$ retrievals, while Figure 6.11 includes the same correlation for HONO.
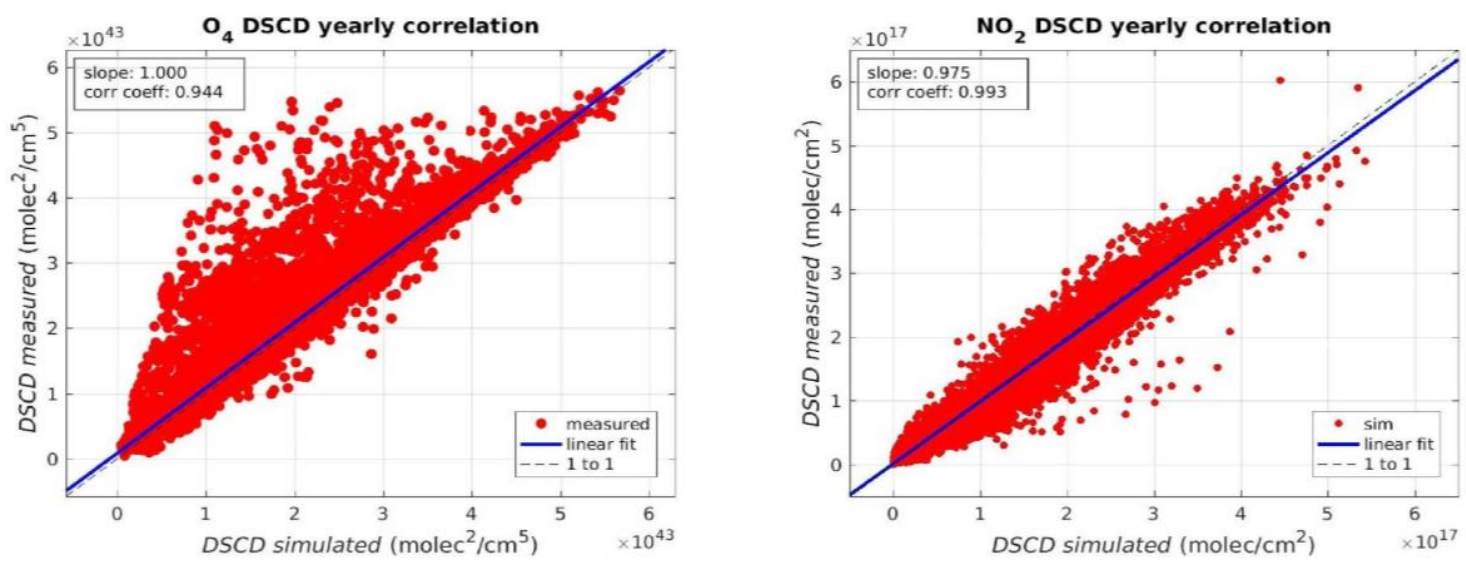

Figure 6.10. Annual correlation of measured and simulated DSCDs of $\mathrm{O}_{4}$ and $\mathrm{NO}_{2}$. 


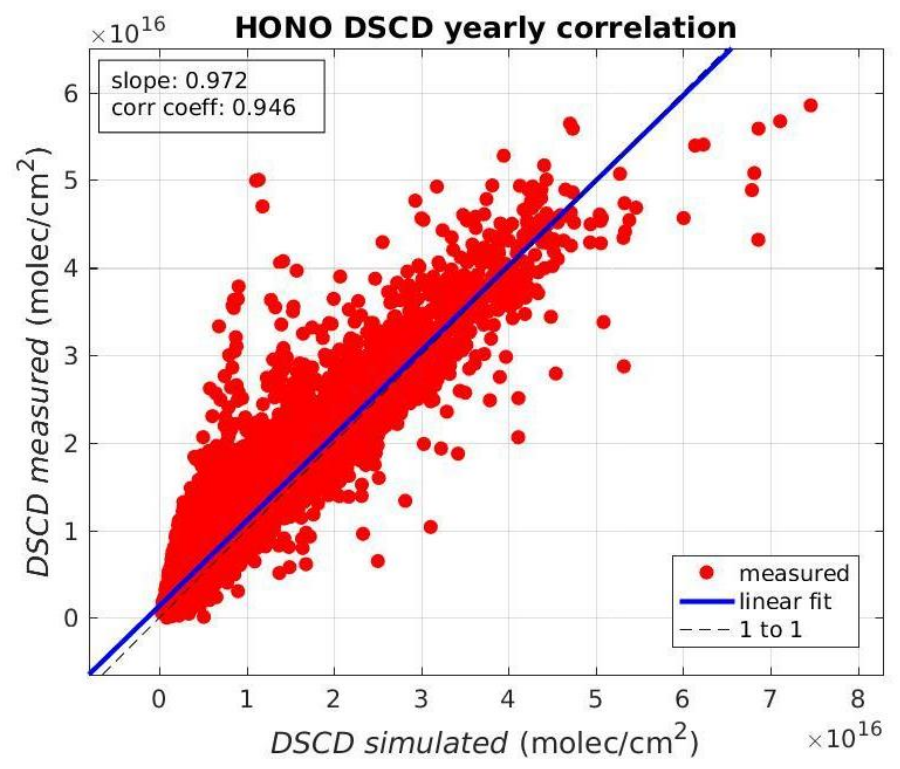

Figure 6.11. Annual correlation of measured and simulated DSCDs of HONO.

Considering that the fit includes an entire year of measurements, these values with slopes close to 1 and correlation coefficients higher than 0.94 offer a solid consistency and agreement. In Figure 6.12, seasonal diurnal variations of HONO are shown. The seasons were split as follows: winter goes from January to March, spring includes April, May and June, while summer encompasses July, August and September; autumn is composed of the remaining months.
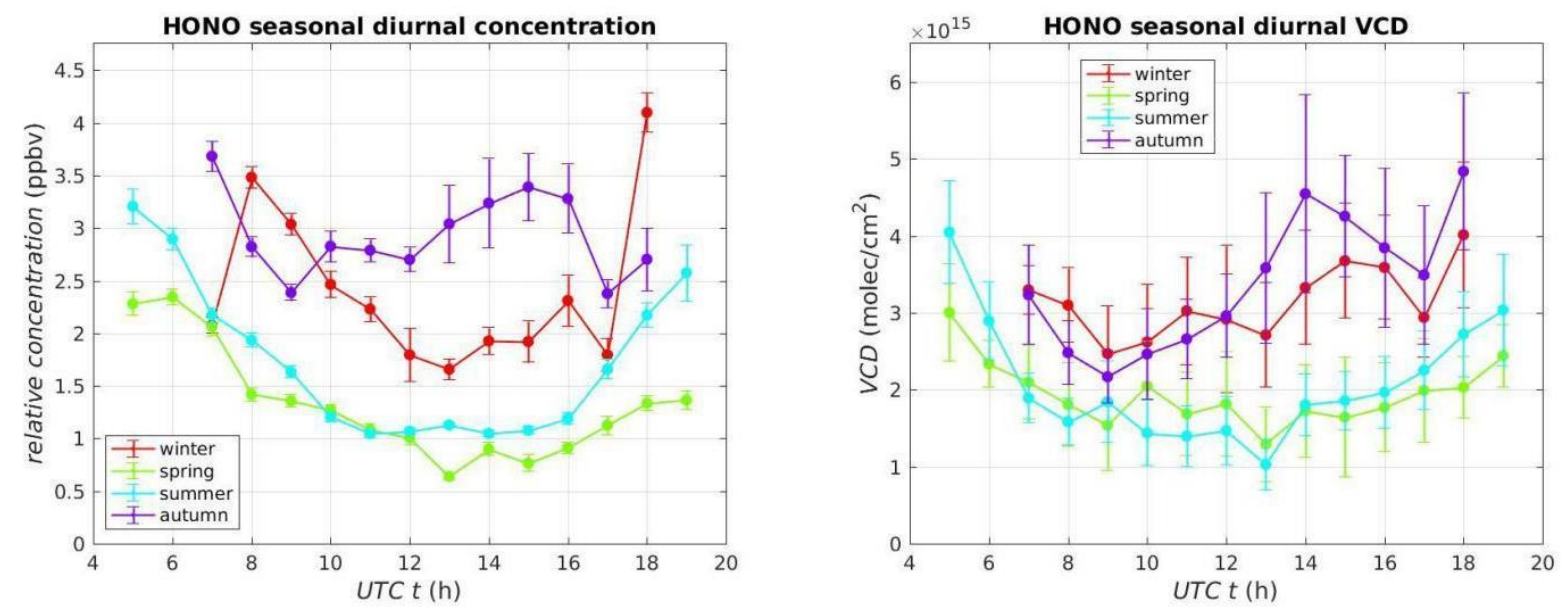

Figure 6.12. Seasonal, diurnal variation of HONO surface mixing ratio and VCDs. 
The left panel in Figure 6.12 shows the HONO mixing ratio in our first layer (up to $100 \mathrm{~m}$ height from the street level) and exhibits maximum values -within each seasonduring the early morning, except for winter, which has a peak of about 4 ppbv near sunset. The impact of photolysis in HONO can be observed, with minimum values around noon, apart from autumn, which presents a more flattened behaviour from 9 UTC on. These relatively constant values could be explained by a less effective photolysis during this period along with an increase in the emissions of precursors (see Figure 6.17) that either directly or indirectly forms HONO, such as the use of house heating. On the other hand, the plot on the right in Figure 6.12 depicts the VCDs, which present similar trends to the relative concentrations, with the higher values in autumn during the afternoon.

Figure 6.13 shows the seasonally averaged diurnal temperature and relative humidity in Madrid during 2016. The measurements come from the air quality stations of the Community of Madrid ("Red de Calidad del Aire de la Comunidad de Madrid") which validate the values daily. Less photolytic loss as the day approaches sunset (diurnal variation), in addition to the decrease in the convective boundary layer height as a function of lower temperatures (as can be seen in the left panel of Figure 6.13), can explain the rise of HONO relative concentrations and VCDs during the autumn and winter evenings. Relative humidity in autumn and winter are higher than in other seasons (Figure 6.13), and these values, along with the Reaction 6.14 could also account for the higher HONO relative concentrations during these seasons. Regarding previous HONO measurements in urban environments, our observed values fall within the order of the reported HONO concentrations, from the first HONO detection ever (Perner and Platt, 1979) up to the most recent works (Hendrick et al., 2014; Acker et al., 2006; Lee et al., 2016), the first layer values are around tenths of ppbv up to $1 \mathrm{ppbv}$ in summer, although some variables such as height of the layer, geolocation and season should be considered for a complete comparison. 

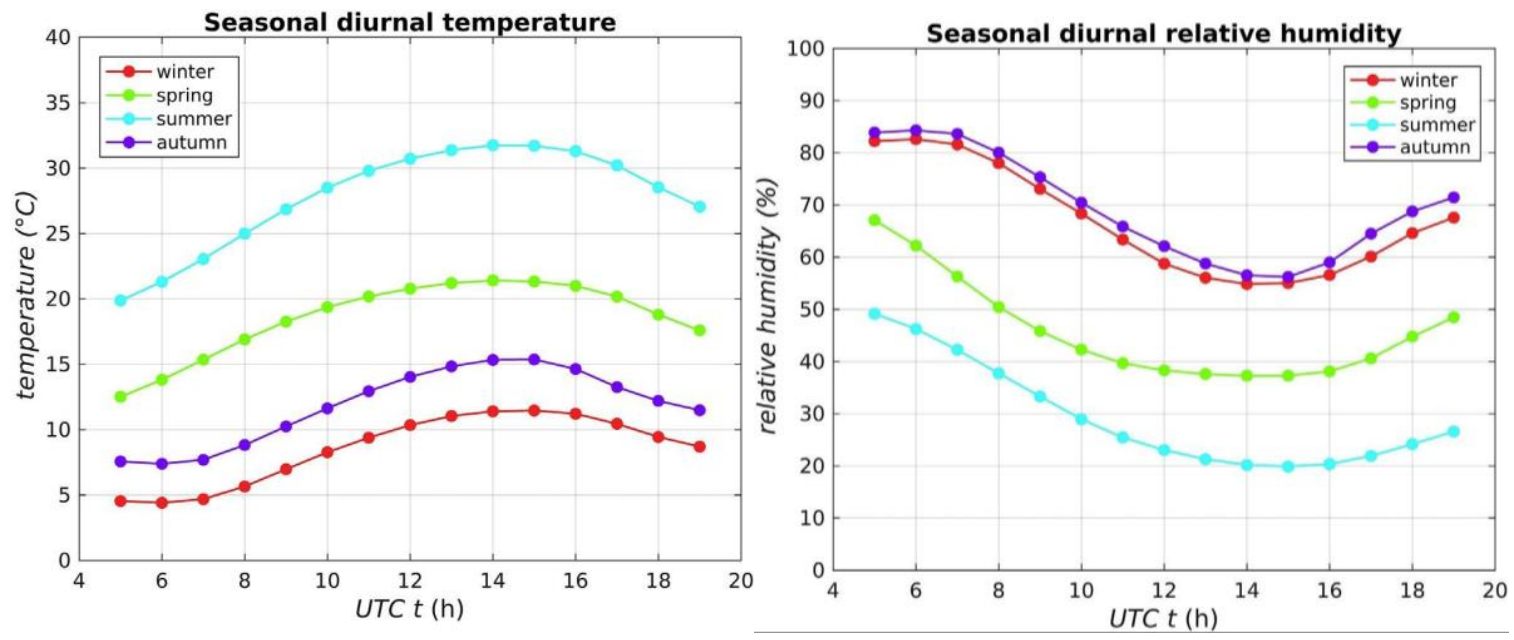

Figure 6.13. Seasonal, diurnal variation of temperature and relative humidity in Madrid during 2016.

Figure 6.14 shows the seasonal average of HONO vertical concentration profiles at 8 UTC, coincident with the early morning traffic rush hour and at a time when the Sun is already above the horizon.

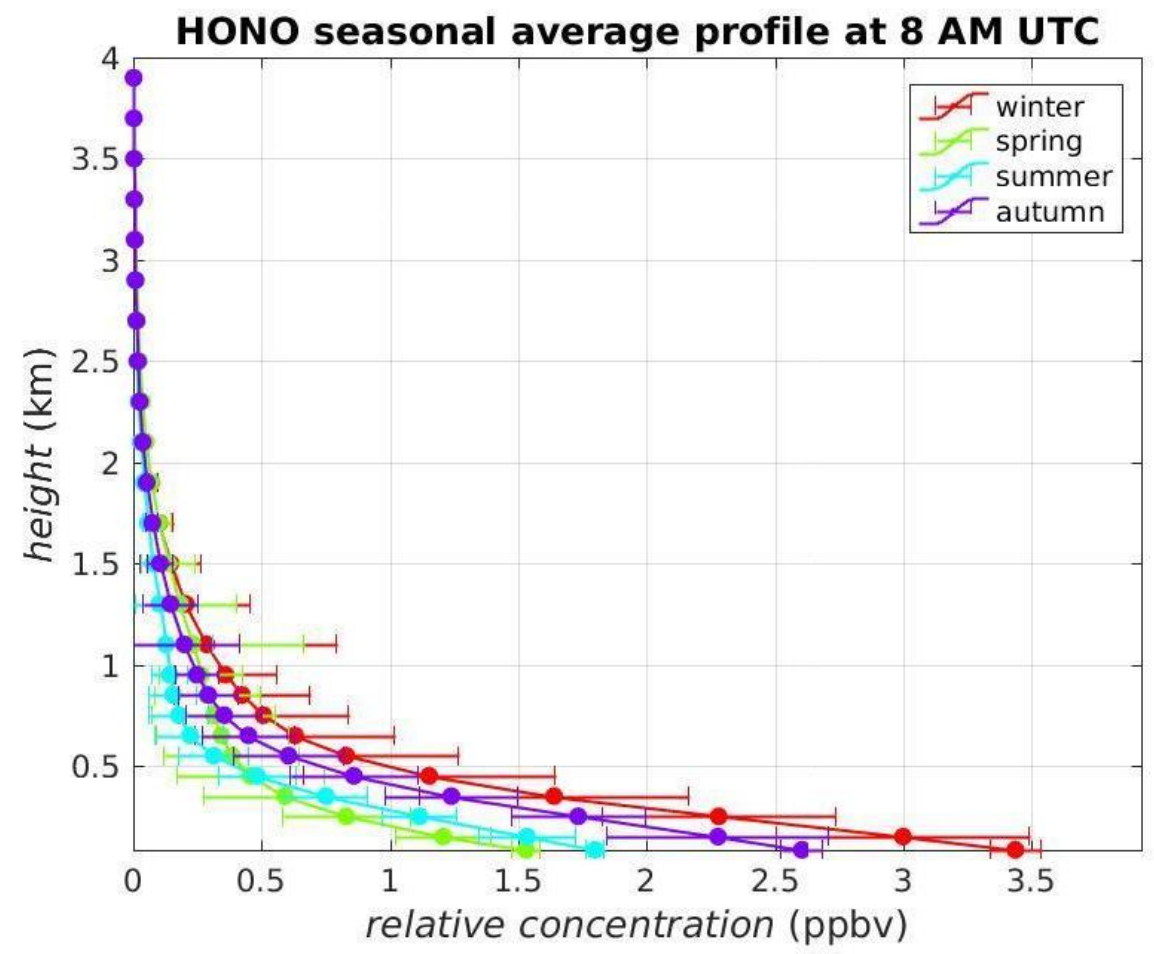

Figure 6.14. Seasonal diurnal profiles of HONO at 8 UTC. 
In Figure 6.14 we can observe that the peak values occur at the lowest level (up to 3.5 ppbv during winter) and then the curves show a fast decrease with height in a fashion similar to exponentially decreasing functions. Note that most of the HONO is located within the first $500 \mathrm{~m}$ from the surface. Moreover, this concentration drop with height is even more pronounced during spring and summer, in accordance with a more active photolysis during those seasons. Moreover, Figs. 6.12 and 6.14 show that there is a marked seasonality in the amounts of HONO, with values consistently higher in autumn and winter than in spring and summer. Figure 6.15 illustrates this seasonal variation for relative concentrations and VCDs during the entire year at 8 UTC.
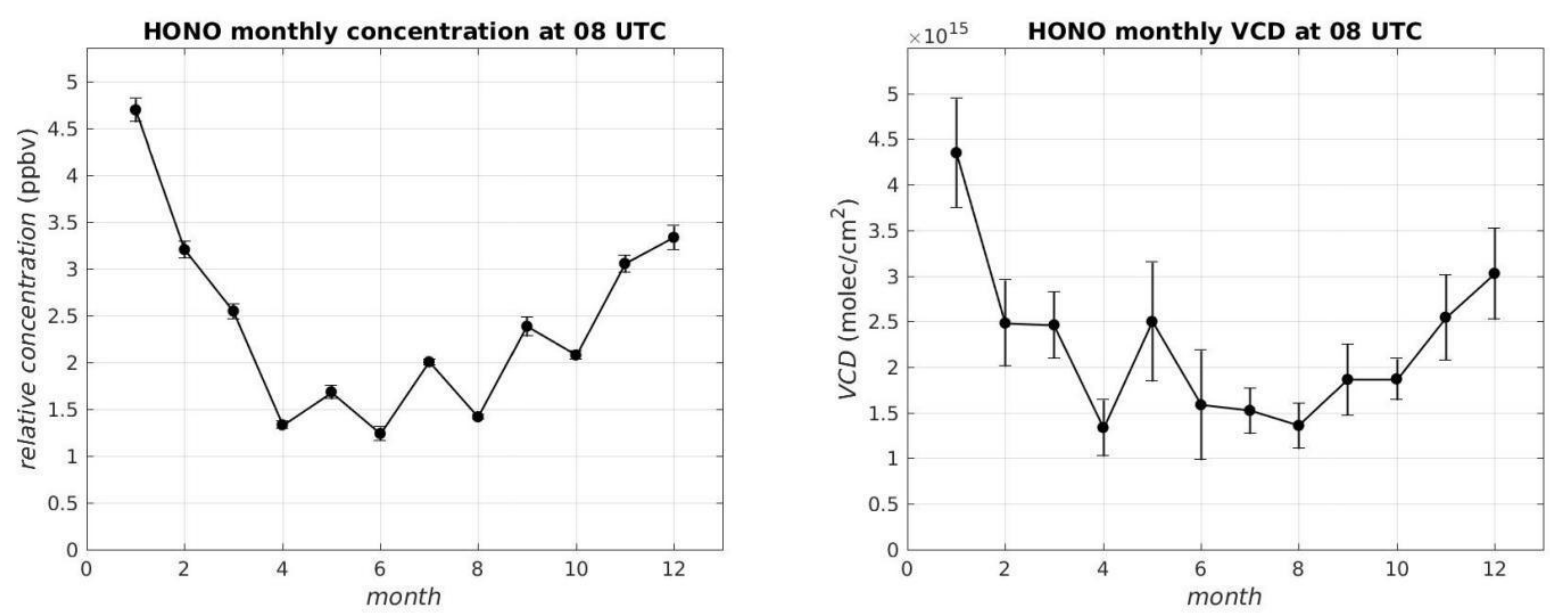

Figure 6.15. HONO monthly variation of relative concentration and VCD at 8 UTC.

The difference between the maximum (January) and the minimum (during summer) is a factor of 3 . This variability can be explained by the large effect of photolysis in summer and the relation between $\mathrm{HONO}$ and $\mathrm{NO}_{2}$, which is thought to be its main precursor. The increase in $\mathrm{NO}_{2}$ levels during autumn and winter is a well-characterised feature of industrialized, polluted urban areas (Richter et al., 2005). This $\mathrm{NO}_{2}$ rise is attributed to the autumn/winter higher emissions, longer photochemical lifetime and lower concentration of $\mathrm{OH}$ radical levels. Figure 6.16 illustrates this seasonal effect for the hourly averaged $\mathrm{NO}_{2}$ relative concentrations within the first layer. Other contributions are the domestic heating in winter and the change in boundary layer height due to different convective conditions, 
which can be of even a factor of 3 -from $1 \mathrm{~km}$ in winter up to $3 \mathrm{~km}$ in summer- leading to a larger dilution of HONO in summer and therefore to lower concentrations close to the surface. However, the latter effect seems to have a minor impact since the relative amplitudes between peaks and troughs in Figures 6.12, 6.14, 6.15 for relative concentrations and VCDs are very similar and they show a reasonably good correlation (0.91).

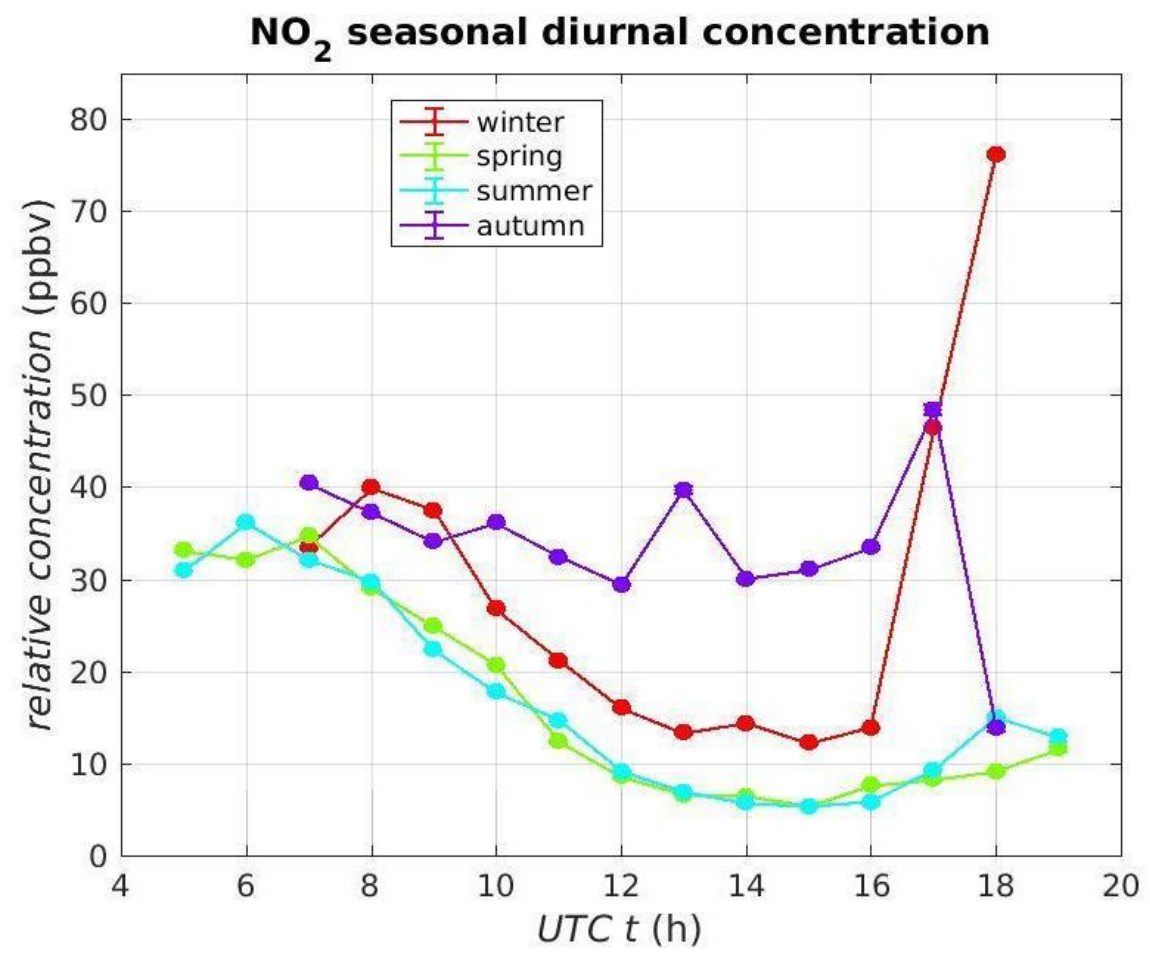

Figure 6.16. Seasonal diurnal variation of $\mathrm{NO}_{2}$ relative concentration (of the first layer).

\subsubsection{Estimation of $\mathrm{OH}$ production from HONO photolysis}

$\mathrm{HONO}$ is one of the main sources of $\mathrm{OH}$ radicals, especially in the early morning, when ozone photolysis is not efficient. Therefore, the evaluation of the $\mathrm{OH}$ production rate from the HONO photolysis is essential to understand the impact of this process on the oxidation capacity of the urban atmosphere. 
We estimate the $\mathrm{OH}$ radical production rate from HONO for the first layer (up to $100 \mathrm{~m}$ height from the ground surface), using the retrieved HONO relative concentrations for that layer and deriving photolysis rates $\operatorname{JONO}(t)$ :

$$
J_{\text {HONO }}(t)=\int_{\lambda_{0}}^{\lambda_{f}} Q Y(\lambda, T) \sigma(\lambda, T) I_{\text {solar }}(\lambda, T) d \lambda
$$

where $Q Y$ is the quantum yield for photolysis, sigma is the respective absorption cross section and $I_{\text {solar }}$ is the solar irradiance (in the form of photon flux) received in an assumed horizontal surface layer. For this particular case, the HONO photolysis $Q Y$ to form $\mathrm{OH}$ is assumed to be 1 (Atkinson et al., 2004), while the photon flux $I_{\text {solar }}$ has been calculated following the spectral irradiance equations of the Sun and taking the evolution of the air mass factor into account (Kasten and Young, 1989). Furthermore, the integral was performed between the same wavelength interval used in the DOAS analysis for HONO (see Table 6.2): 337-375 $\mathrm{nm}$.

Once $\operatorname{JHONO}_{\mathrm{HON}}(t)$ is obtained, it is crossed with the corresponding first layer concentration vector (which also depends on time). Later on, the results have been hourly and seasonally averaged (Figure 6.17).

The $\mathrm{OH}$ radical production exhibits its maximum values at around noon, with the absolute peak occurring in autumn at 13 UTC time. It is also noticeable that in the early morning ( 5 to 9 UTC) and late afternoon (16 to 19 UTC) the contribution of HONO to the $\mathrm{OH}$ radical is higher during spring and summer, mainly due to the more effective photolysis in these periods. Both the shape and the values of $\mathrm{OH}$ production are similar to the ones calculated in previous studies (Alicke et al., 2003). 


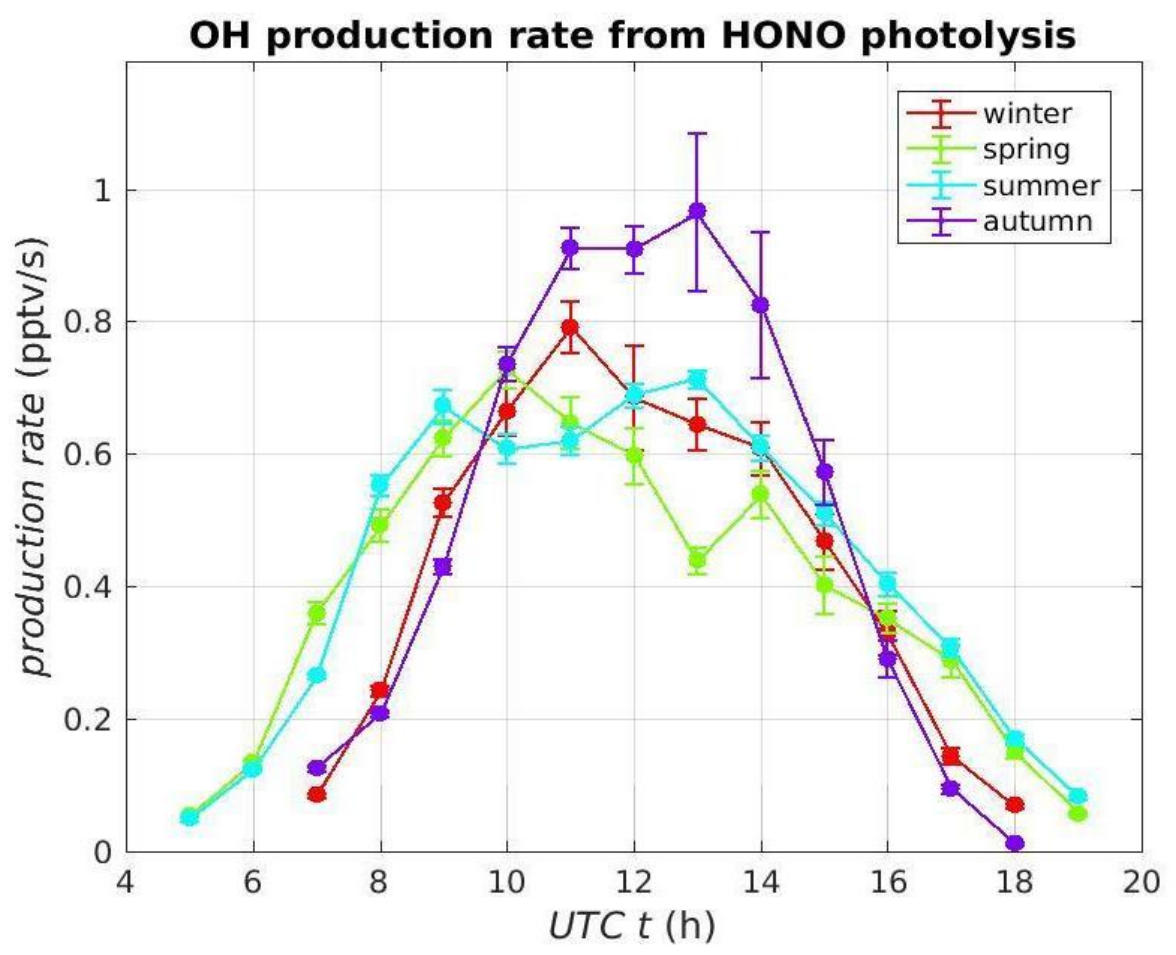

Figure 6.17. Seasonal and diurnal $\mathrm{OH}$ production rate from $\mathrm{HONO}$ photolysis. 


\section{GLYOXAL AND HCHO}

In this chapter the results for glyoxal and formaldehyde are described. First, an introduction about the context and basic chemical reactions for both trace gases is provided, followed by the DOAS analysis, the inversion parameters and the results. The findings in this chapter have been published in Benavent et al., 2019.

\subsection{Introduction}

Glyoxal and formaldehyde are organic trace gases that play an important role in tropospheric chemistry as oxidation products of a number of Volatile Organic Compounds (VOCs).

VOCs play an important function in the atmosphere despite their low concentrations. Their photolysis contributes to the formation of $\mathrm{O}_{3}$ and SOAs (Gouw et al., 2018). They are emitted from different sources including the oceans, fossil fuel burning, biomass burning and geochemical processes (Vrekoussis et al., 2010). Urban atmospheric VOCs have an atmospheric lifetime in the order of days (Garzon et al., 2015) and their total emission uncertainties are high because they depend on factors such as temperature, humidity, pressure and motor vehicle emissions exhaust.

Formaldehyde and glyoxal can act as traces of VOCs oxidation. One of the most important reactions of these VOCs is the oxidation by $\mathrm{O}_{3}$ or $\mathrm{OH}$ resulting in significant channels of $\mathrm{HCHO}$ and $\mathrm{CHOCHO}$ production. Oxidation of isoprene, alkyne and aromatic hydrocarbons generates glyoxal, which is the simplest $\alpha$-dicarbonyl (Volkamer et al., 2005a). Its most important removal process in the atmosphere is photolysis (Reactions R7.1, R7.4) and reaction with $\mathrm{OH}$ radicals (Reactions R7.5, R7.6). Glyoxal has a lifetime of 1- 
$2 \mathrm{~h}$ in presence of sunlight (Sinreich et al., 2010). CHOCHO also plays an important role in SOA formation and it has been suggested to have an impact on the Cloud Condensation Nuclei (CCN) formation (Vrekoussis et al., 2010).

$$
\begin{aligned}
& \mathrm{CHOCHO}+\mathrm{hv}-->2 \mathrm{HCO} \\
& \mathrm{CHOCHO}+\mathrm{hv}->\mathrm{H}_{2}+2 \mathrm{CO} \\
& \mathrm{CHOCHO}+\mathrm{hv}->\mathrm{H}_{2} \mathrm{CO}+\mathrm{CO} \\
& \mathrm{CHOCHO}+\mathrm{hv}->\mathrm{H}+\mathrm{CO}+\mathrm{HCHO} \\
& \mathrm{CHOCHO}+\mathrm{OH}-->\mathrm{HCOCO}+\mathrm{H}_{2} \mathrm{O} \\
& \mathrm{HCOCO}+\mathrm{O}_{2}->2 \mathrm{CO}+\mathrm{HO}_{2}
\end{aligned}
$$

Formaldehyde is produced by the photo-oxidation of VOCs which are released to the atmosphere from different natural and anthropogenic sources. However, background levels of $\mathrm{HCHO}$ are regulated by the total sum of tropospheric $\mathrm{CH}_{4}$ (Gratsea et al., 2016):

$$
\begin{aligned}
& \mathrm{OH}+\mathrm{CH}_{4}-->\mathrm{CH}_{3}+\mathrm{H}_{2} \mathrm{O} \\
& \mathrm{CH}_{3}+\mathrm{O}_{2}+\mathrm{M}-->\mathrm{CH}_{3} \mathrm{O}_{2}+\mathrm{M} \\
& \mathrm{CH}_{3} \mathrm{O}_{2}+\mathrm{NO}-->\mathrm{NO}_{2}+\mathrm{CH}_{3} \mathrm{O} \\
& \mathrm{CH}_{3} \mathrm{O}+\mathrm{O}_{2}-->\mathrm{HCHO}+\mathrm{HO}_{2}
\end{aligned}
$$


The lifetime of $\mathrm{HCHO}$ is about $2 \mathrm{~h}$ (Palmer et al., 2003), mainly due to its significant photolysis in the UV region (see Reactions R7.11 and R7.12) and reaction with hydroxyl radicals (Reactions R7.13 and R7.15) (Dufour et al., 2009).

$$
\begin{aligned}
& \mathrm{HCHO}+\mathrm{hv}->\mathrm{H}_{2}+\mathrm{CO} \\
& \mathrm{HCHO}+\mathrm{hv}-->\mathrm{H}+\mathrm{HCO} \\
& \mathrm{HCHO}+\mathrm{OH}-->\mathrm{HCHO}+\mathrm{H}_{2} \mathrm{O} \\
& \mathrm{HCHO}+\mathrm{O}_{2}->\mathrm{HO}_{2}+\mathrm{CO} \\
& \mathrm{HO}_{2}+\mathrm{NO}-->\mathrm{NO}_{2}+\mathrm{OH}
\end{aligned}
$$

The ratio of $\mathrm{CHOCHO}$ to $\mathrm{HCHO}$ can be used as an indicator of the nature of VOCs precursors (Kaiser et al., 2015). As mentioned before, CHOCHO is formed from alkenes, aromatics, isoprene and monoterpenes and $\mathrm{HCHO}$ is an oxidation product of pyrogenic and anthropogenic VOCs. The two oxidation products have similar lifetimes in the atmosphere and they can be used as an indicator of the speciation of VOCs contributing to the total VOC reactivity. For instance, high $R_{G F}$ has been related to biogenic emissions, while low $R_{G F}$ is usually linked with anthropogenic emissions although it is worth noting that there are some discrepancies related to this point (DiGangi et al., 2012).

Considering the relevance of VOCs in tropospheric chemistry, oxidation capacity and the role of $\mathrm{CHOCHO}$ and $\mathrm{HCHO}$ as VOCs source emission tracers, more measurements of these species in large cities are still needed. Moreover, in the particular atmosphere of Madrid, a recent increase in oxidation capacity has been proposed following the reduction in $\mathrm{NO}_{\mathrm{x}}$ emissions due to the recent economic recession (Saiz-Lopez et al., 2017). This 
potential change in the burden of oxidants such as $\mathrm{OH}$ and $\mathrm{O}_{3}$ may have an impact on the atmospheric abundances of VOCs and in turn, in that of $\mathrm{HCHO}$ and $\mathrm{CHOCHO}$ in Madrid.

Both formaldehyde and glyoxal have strong, and narrow, absorption bands in the UV and VIS wavelength range, respectively, which have previously been used to measure these species in urban areas (e.g. Heckel et al., 2005; Pinardi et al., 2013; Volkamer et al., 2005a). In this work, we present the year-round measurements of glyoxal and formaldehyde in the city of Madrid, Spain, using a MAXDOAS instrument. One year, January 1 to December 31, 2016, of $\mathrm{HCHO}$ and $\mathrm{CHOCHO}$ observations is used to study their surface concentrations, vertical distributions, and diurnal and seasonal evolution.

\subsection{Experimental}

For the glyoxal and formaldehyde analysis, our MAXDOAS-1D instrument was again used (detailed in Chapter 5).

In particular for the glyoxal retrieval, and considering the difficulties of the analysis mainly due to water vapor interference (Mahajan et al., 2014), several sensitivity analysis procedures have been conducted in order to quantify any systematic error. The polynomial degree, the wavelength range and the intensity offset were varied. A small wavelength gap was also used to avoid the strong water absorption features (442-229 nm) instead of using the whole spectral window (424-457 nm). Moreover, the inclusion of an averaged residual like an "instrumental" cross section with the purpose of taking care of possible systematic structures in our spectra has been tried, but the results did not improve. Every combination of the mentioned parameters was applied to the following clear sky days: January 1, 2016; April 6, 2016; July 1, 2016 and September 1, 2016. Finally, the best configuration found (with the lowest residual RMS and a good spectral fitting) was the following: wavelength range of 425-457 $\mathrm{nm}$ with a gap between 442 and $449 \mathrm{~nm}$. There are some discrepancies about the wavelength region used in the glyoxal DOAS analysis: Sinreich et al., 2007 used a 
wavelength between 420 and $460 \mathrm{~nm}$; Coburn et al., 2014 applied a wavelength range of 433-460 nm, and the 434-460 nm window was used in Khokhar et al., 2016.

For $\mathrm{HCHO}$, the wavelength range used in this study follows that of previous reports: Heckel et al., 2005 applied a fitting window between 335 and 357 nm, 336.5-359 nm was used in Pinardi et al., 2013; 336-358 nm in Vigouroux et al., 2009 and 336-359 nm in Irie et al., 2011. Absorption cross sections included in this study are shown in Table 7.1 for glyoxal (based on Mahajan et al., 2014) and Table 7.2 for formaldehyde (configuration used in the CINDI-2 campaign, http://www.tropomi.eu/data-products/cindi-2). 
Table 7.1. DOAS fit settings for the retrieval of glyoxal in the VIS range.

\begin{tabular}{|l|l|}
\hline \multicolumn{1}{|c|}{ Parameter } & \multicolumn{1}{|c|}{ Value } \\
\hline Fitting window & $424-457 \mathrm{~nm}$ \\
\hline Wavelength calibration & $\begin{array}{l}\text { Based on reference solar atlas (Chance and } \\
\text { Kurucz, 2010). }\end{array}$ \\
\hline Zenith reference & Scan \\
\hline Polynomial order & 2 \\
\hline Intensity offset & - \\
\hline Shift & All measured spectra and the Ring cross \\
& section were allowed to shift and stretch \\
(order 1) in wavelength.
\end{tabular}


Table 7.2. DOAS fit settings for the retrieval of $\mathrm{HCHO}$ in the UV range.

\begin{tabular}{|c|c|}
\hline Parameter & Value \\
\hline Fitting window & $336.5-359 \mathrm{~nm}$ \\
\hline Wavelength calibration & $\begin{array}{l}\text { Based on reference solar atlas (Chance and } \\
\text { Kurucz, 2010). }\end{array}$ \\
\hline Zenith reference & Scan \\
\hline Polynomial order & 3 \\
\hline Intensity offset & Order 1 \\
\hline Shift & $\begin{array}{l}\text { All measured spectra and the Ring cross } \\
\text { section were allowed to shift and stretch } \\
\text { (order } 1 \text { ) in wavelength. }\end{array}$ \\
\hline Molecule & Cross section \\
\hline $\mathrm{HCHO}$ & 297 K (Meller and Moortgat, 2000) \\
\hline $\mathrm{O}_{4}$ & $293 \mathrm{~K}$ (Thalman and Volkamer, 2013) \\
\hline $\mathrm{NO}_{2}$ & 298 K (Vandaele et al., 1998) \\
\hline $\mathrm{O}_{3} \mathrm{a}$ & $223 \mathrm{~K}$ (Serdyuchenko et al., 2014) \\
\hline $\mathrm{O}_{3} \mathrm{~b}$ & $243 \mathrm{~K}$ (Serdyuchenko et al., 2014) \\
\hline $\mathrm{BrO}$ & 223 K (Fleischmann et al., 2004) \\
\hline Ring & Calculated by QDOAS \\
\hline
\end{tabular}


The analysis of $\mathrm{O}_{4}$, which is needed for the aerosol extinction profile retrieval, was done in the UV region. It uses the range of $338-370 \mathrm{~nm}$ and the configuration used in Chapter 6.

\subsection{Analysis methods}

\subsubsection{Cloud screening and quality filters}

In order to avoid the influence of cloudy skies, we applied the cloud screening as it is explained in Subsection 4.5. Before using the profile retrieval algorithm, some quality filters were applied (explained in Subsection 4.7) in order to avoid not reliable DSCDs. Once these filters were applied, the remaining days were: 269 for $\mathrm{HCHO}$ and 249 for $\mathrm{CHOCHO}$. By season, there are 58 days in winter, 69 in spring, 85 in summer and 57 in autumn for formaldehyde, and 49 days in winter, 64 days in spring, 80 days in summer and 56 days in autumn for glyoxal.

\subsubsection{Spectral detection}

The complete (and already filtered) collection of scattered light spectra are prepared for the next step. Two examples of the spectral fittings for $\mathrm{HCHO}$ and $\mathrm{CHOCHO}$ appear in Figure 7.1. The zenith measurement of each MAXDOAS scan has been used as the reference spectrum. 

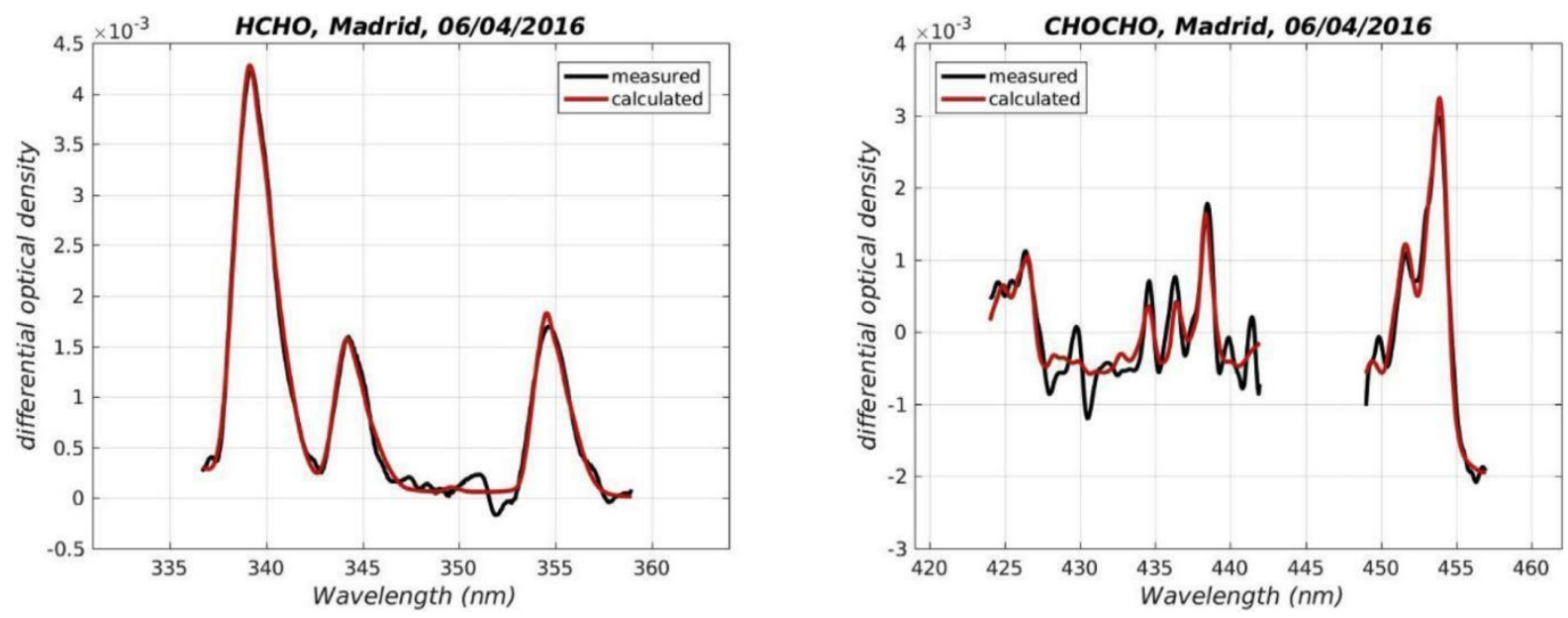

Figure 7.1. Formaldehyde and glyoxal spectral fitting examples. The red curves are the calculated optical densities for each trace gas (using QDOAS) while the black curves represent the measured optical density and the residual of the analysis, and they stand for the goodness of the analysis. The spectrum was collected on April 6,2016 , at a VEA of $20^{\circ}$ and a SZA of $54^{\circ}$ for formaldehyde and at a VEA of $20^{\circ}$ and SZA of $65^{\circ}$ for glyoxal.

\subsubsection{Profile retrieval configuration}

Additional information on the calculation of AODs and extinction coefficients for this study is detailed in Chapter 6. Making use of the extinction coefficient profiles derived from the UV region (at $360 \mathrm{~nm}$ ) and the Angstrom exponent ( $\alpha$ ) (available on the AERONET website) we calculated the extinction coefficient in the visible region following this equation:

$$
\tau_{455}=\tau_{360}\left(\frac{\lambda_{455}}{\lambda_{360}}\right)^{-\alpha}
$$

where $\tau$ is the extinction coefficient, $\lambda$ is the wavelength and $\alpha$ is the Ångstrom exponent between $380 \mathrm{~nm}$ and $500 \mathrm{~nm}$. The wavelength of $455 \mathrm{~nm}$ comes from the maximum differential absorption coefficient of glyoxal.

The a priori profiles and their associated covariance matrices have a noticeable effect in the results. A balance has to be found to optimize the degrees of freedom of the 
analysis while avoiding non-physical oscillations. The relative weight of the a priori profiles in the analysis comes from the covariance matrix. In this study, a scaling factor of 0.5 times the a priori profile squared, with a correlation length between layers of $0.2 \mathrm{~km}$ has been chosen (see Clémer et al., 2010).

\subsection{Results and discussion}

\subsubsection{HCHO profiles}

Following an analogous idea as in Chapter 6, Figure 7.2 represents the long-term correlation for simulated and measured HCHO DSCDs throughout the year. The slope of the fit is 0.89 , with a correlation coefficient higher than 0.95 . These values reflect a reliable and consistent fit quality.

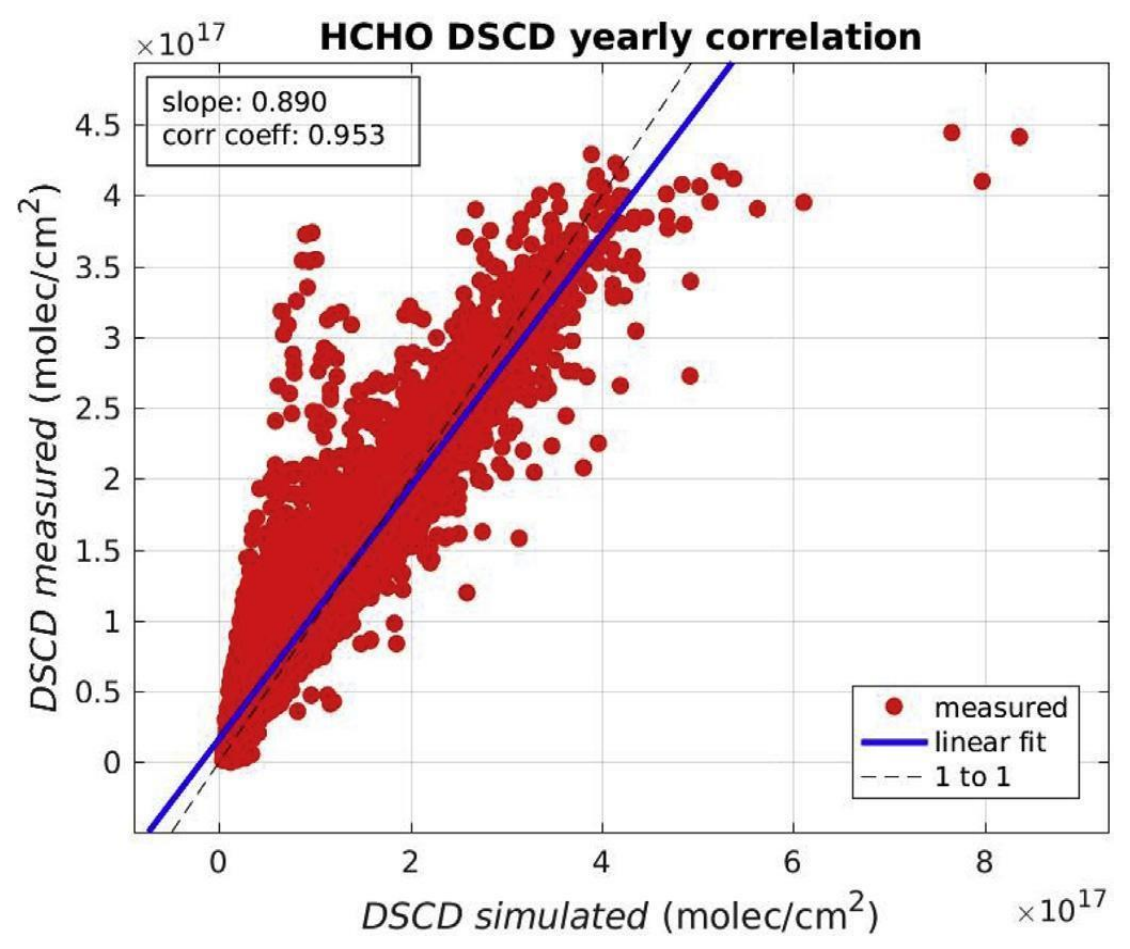

Figure 7.2. Long-term correlation of simulated and measured HCHO DSCDs throughout the year. 
Figure 7.3 shows an example of $\mathrm{HCHO}$ profiles on April 6, 2016 at an hourly basis. Panels a) and b) demonstrate the good correlation between DSCDs (even considering the relatively large uncertainties at low VEAs), with a correlation coefficient value very close to 1. The lower panel includes mixing ratio results: the diurnal vertical profile for this day (Figure $7.3 \mathrm{c}$ ) and the temporal evolution of the mixing ratio in the first layer (up to $100 \mathrm{~m}$ height) (Figure $7.3 \mathrm{~d}$ ). Madrid typically experiences two peaks of traffic rush hours, the first one around 8-9 local time and the second at 19-20 local time (Borge et al., 2016). Both peaks, for April 6, are located at 6-7 and 17-18 UTC time, respectively. Figure 7.3 shows that both traffic peaks coincide with the highest levels of $\mathrm{HCHO}$, with a maximum surface mixing ratio value of about $30 \mathrm{ppbv}$ in the early morning (at 6 UTC). Note that surface mixing ratio levels around noon are rather steady and they seem to be well-mixed within the first hundred meters, with values of roughly $7 \mathrm{ppbv}$, which is in accordance with the relatively slow photolysis rate of $\mathrm{HCHO}$ and its lifetime in the order of a few hours. We estimated the vertical concentration profile to decay roughly $90 \%$ within the first $\mathrm{km}$, while the temporal decay -between the rush hour and noon time- is of the order of $-3.5 \mathrm{ppbv} \mathrm{h}^{-1}$ for this day. 

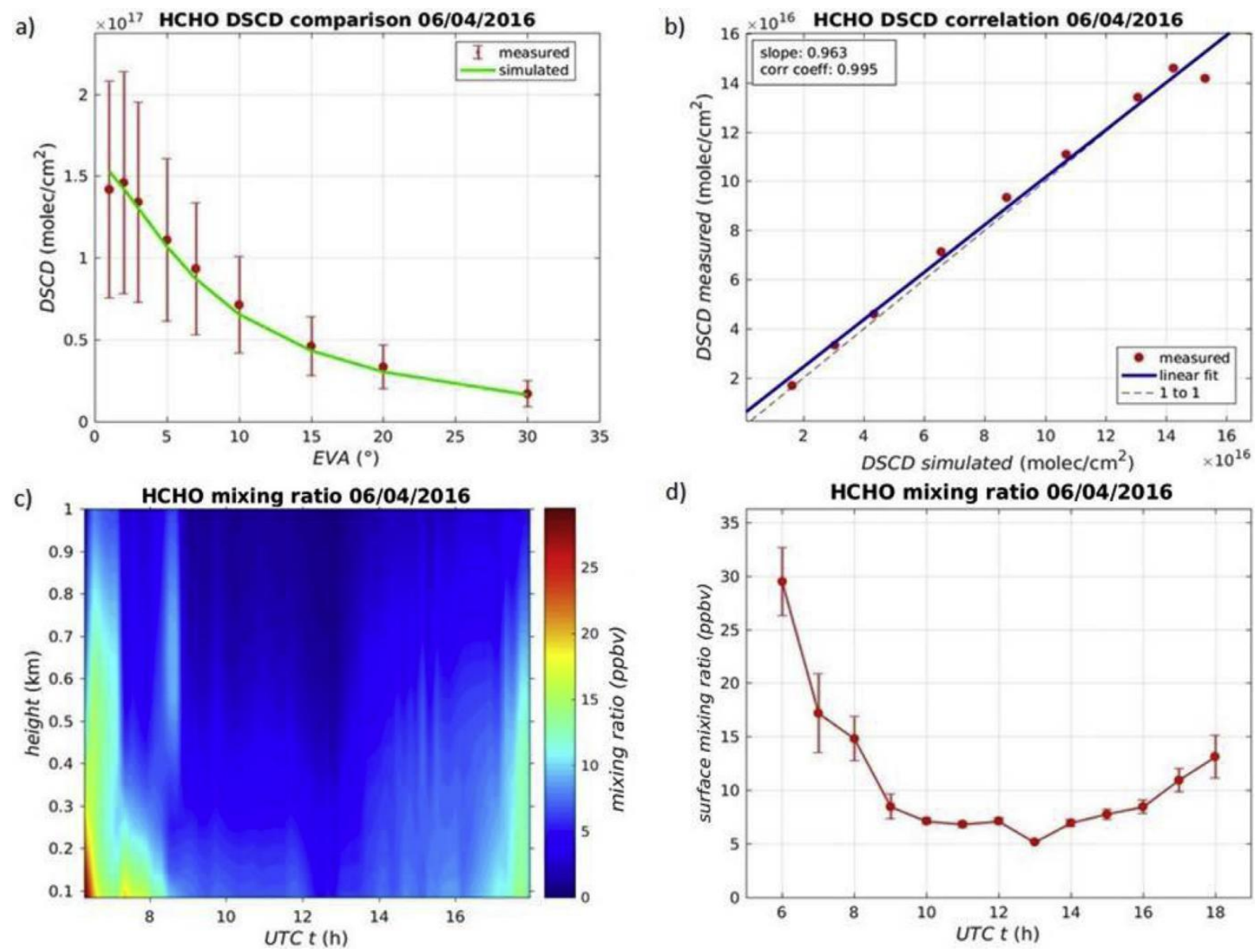

Figure 7.3. a) Measured and simulated HCHO DSCDs comparison, b) correlation between measured and simulated $\mathrm{HCHO}$ DSCDs, c) diurnal vertical profiles of $\mathrm{HCHO}$ mixing ratio d) $\mathrm{HCHO}$ surface mixing ratio. The measurements of these plots were all taken on April 6, 2016.

Since an entire year of measurements is available, it is possible to assess seasonal variations in the levels of these trace gases. $\mathrm{HCHO}$ seasonal daytime series of surface mixing ratios and VCDs are shown in Figure 7.4. The curves correspond to the average diurnal variation for each season. It can be seen that both variables have similar trends for every season. It is also observed that the curves do not exhibit swift temporal changes, which is in accordance with the relatively slow $\mathrm{HCHO}$ photolysis. However, values during autumn and winter are higher than in spring and summer, due to the lower photolysis rates and more effective accumulation of $\mathrm{HCHO}$ in autumn and winter. Seasonal assessments of $\mathrm{HCHO}$ measurements in urban environments have been previously reported in the 
literature, showing different trends depending on the place (Sin et al., 2001): for instance, maximum levels of $\mathrm{HCHO}$ in autumn and winter were found in Grosjean, 1991 and Bakeas et al., 2003, while other studies reported higher values in spring and summer (Possanzini et al., 1996; Ho et al., 2002a,b). It is widely accepted that a main formation channel for carbonyls like $\mathrm{HCHO}$ is the photochemical oxidation of hydrocarbons as well as the direct emissions from vehicles. Assuming these vehicular emissions to be relatively steady in Madrid (in terms of seasonality at a given time of the day), other secondary sources could play a role in the seasonal evolution of atmospheric HCHO. As explained in Sin et al., 2001, although higher temperatures and solar irradiance levels may increase the photochemical production during spring and summer, they also favour the photolysis rates that produce $\mathrm{OH}$, which ends up being an additional sink of $\mathrm{HCHO}$. Thus the net result at each moment will be determined by the balance between photochemical production and removal processes. Figure 7.4 shows the seasonal, hourly-averaged diurnal surface mixing ratio (within the first layer: 0-100 m) of HCHO.

For each season we observe two peaks, in the early morning and evening during spring and summer, while for autumn and winter the second peak moves approaching noon, resulting also in higher values than in spring and summer. Three main reasons can account for these generally higher levels of $\mathrm{HCHO}$ during autumn and winter, especially in the afternoon: the decrease in solar irradiance levels, thus reducing the photolysis of $\mathrm{HCHO}$, the rise of direct emissions and precursors (for instance house heating) and the contraction of the boundary layer height during cold seasons, which enhance the pollutants accumulation increasing their mixing ratios close to the surface. Overall, the lowest surface mixing ratio -of about 6 ppbv- was found in spring at 13 UTC, while the maximum value, $\sim 30$ ppbv, is measured in autumn at 07 UTC. 

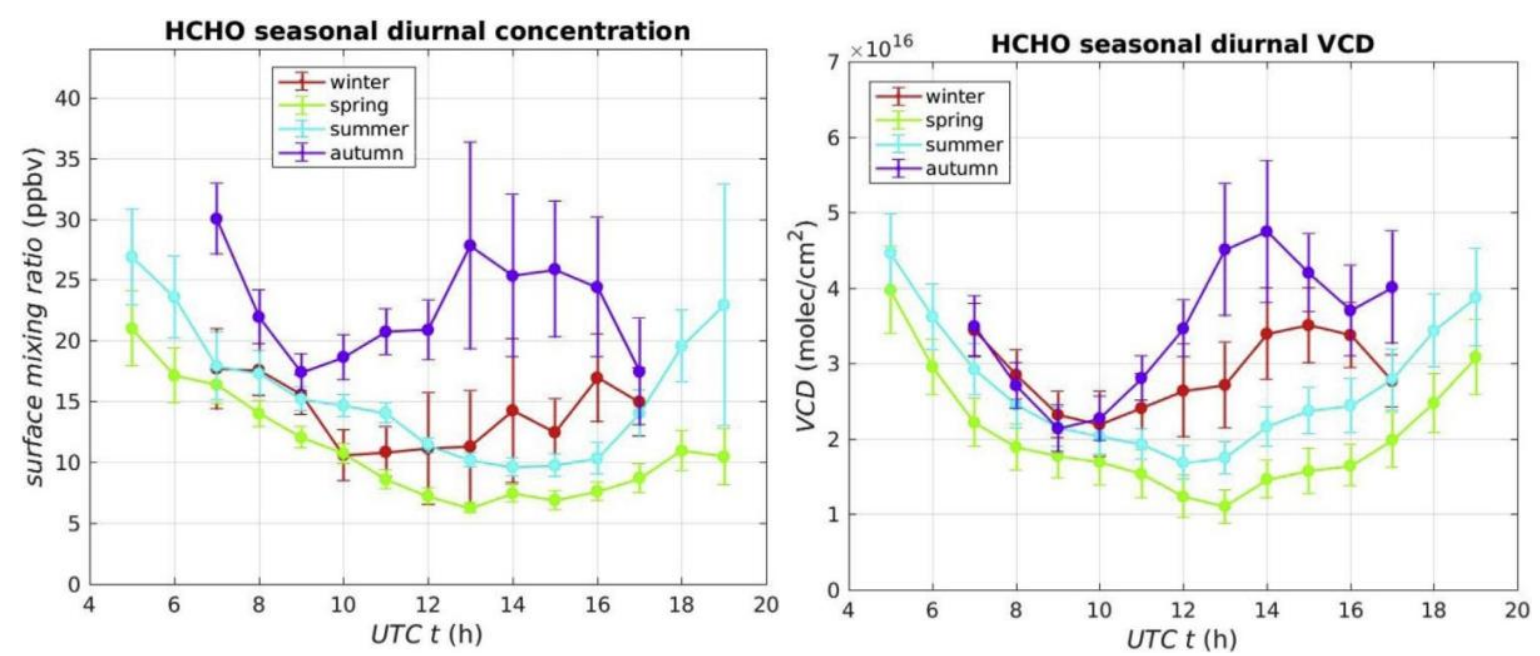

Figure 7.4. Seasonally averaged diurnal surface mixing ratios and VCDs of HCHO.

VCDs present similar seasonal trends to the surface mixing ratios: two peaks during the day for each season and higher values in autumn and winter, especially during the afternoon. This similarity can be expected since the tropospheric $\mathrm{HCHO}$ column over Madrid is dominated by the $\mathrm{HCHO}$ levels in the boundary layer. In spring and summer the VCD values span between $1 \times 10^{16}$ molecules $\mathrm{cm}^{-2}$ (around noon) and $4.5 \times 10^{16}$ molecules $\mathrm{cm}^{-}$ 2 (in the early morning) while during autumn and winter the VCDs range from $2 \times 10^{16}$ molecules $\mathrm{cm}^{-2}$ (at 9 UTC) to $5 \times 10^{16}$ molecules $\mathrm{cm}^{-2}$ (at 14 UTC). Recent studies (Lee et al., 2015) have found similar HCHO ranges of surface mixing ratios and VCDs in urban sites using the MAXDOAS technique.

Figure 7.5 includes monthly hourly-averages of the $\mathrm{HCHO}$ first layer mixing ratio and VCDs at certain hours during the day. In the morning (at 09 UTC, upper panel) there is not a marked seasonality in either variable (smooth trends with maximum differences below a factor of 2), whereas there is indeed a more noticeable temporal change at noon (12 UTC, medium panel), when both the irradiance and photolysis levels are higher. At this time a peak-valley difference of a factor of 6 in surface mixing ratios and of a factor of 4 in VCDs can be observed. In addition, in the afternoon (at 16 UTC, lower panel) we found again considerable monthly variations (maximum differences of a factor of 5 and 3 in surface mixing ratios and VCDs, respectively). The last two panels of Figure 7.6 shows an analogous 
behaviour as in Figure 7.5 for the afternoon $\mathrm{HCHO}$ levels in autumn and winter months, which can also be explained by i) the combination of a less effective photolysis, along with the decrease in the boundary layer height during those seasons (e.g. can even be a factor of 3, with the associated increase in $\mathrm{HCHO}$ accumulation) and ii) the increase in direct emissions during winter which can exceed the effect of the more intense photochemical activity in spring and summer.

There are several studies of HCHO using satellite measurements (Gonzalez-Abad et al., 2015). We have used for comparison purposes the monthly averaged OMI results from 2016 and we observed that there is not a clear seasonal variability (also found in GonzalezAbad et al., 2015) while our MAXDOAS VCDs (Figure 7.4) show seasonal changes around the passing time of the satellite over Madrid. Taking yearly averages, the MAXDOAS VCDs are $67 \%$ higher than the OMI values (a similar difference in an urban environment was reported in Lee et al., 2015). This difference could be explained by two reasons: we considered the typical pixel size ( $21 \times 28 \mathrm{~km}$ for OMI in Madrid) to be larger than our spatial scale for comparison purposes (note that our MAXDOAS measurements are representative for distances of approximately $10 \mathrm{~km}$ within the viewing direction), moreover these pixels are not able to distinguish local emission sources from the total amount measured in each pixel. 

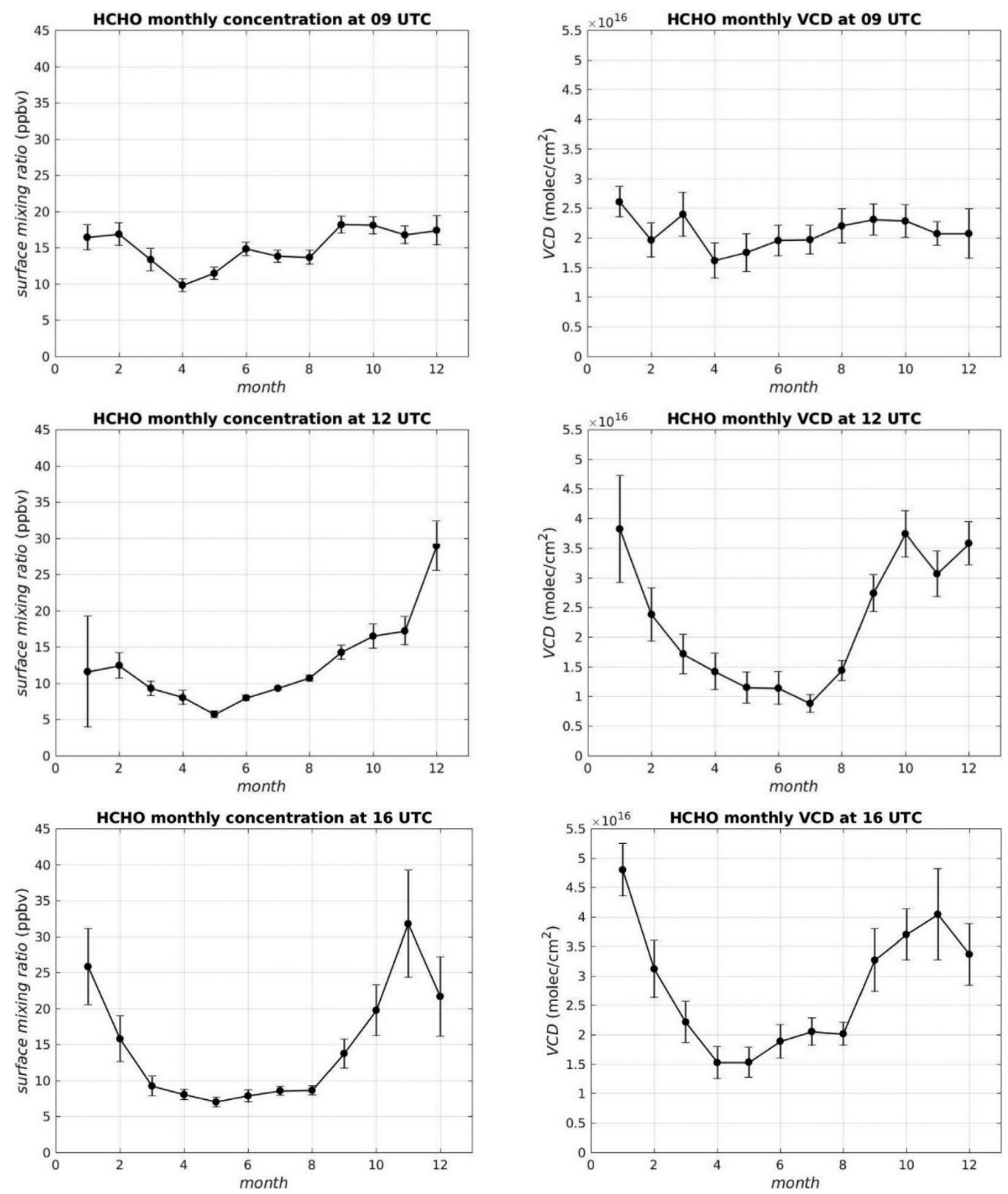

Figure 7.5. Monthly variation of hourly averaged surface mixing ratios (left column) and VCDs (right column) for $\mathrm{HCHO}$ at three different UTC hours. 


\subsubsection{Glyoxal profiles}

We now explore the glyoxal vertical concentration profiles. As for $\mathrm{HCHO}$, Figure 7.7 includes the comparison between the measured and simulated DSCDs of CHOCHO. It can be noted that the comparison is not as robust as for $\mathrm{HCHO}$, and we find higher DSCD uncertainties for low VEAs. As mentioned in Section 2.1, $\mathrm{CHOCHO}$ is a complicated trace gas to be retrieved using DOAS, particularly because of the interference of water vapor absorptions. Note also that this is one of the still few glyoxal DOAS analyses in the literature. In Figure 7.6 there is a glyoxal profile for November 10, 2016. First layer concentrations peak at 10 UTC, at around $1.2 \mathrm{ppbv}$, and reach a minimum value at 14 UTC, close to 0.4 ppbv (Figure 7.6). This yields a temporal decay for this time interval of about 0.25 pptv $\mathrm{h}^{-1}$. We calculated that around $85-90 \%$ of the glyoxal total column is located within the first kilometer.

a)

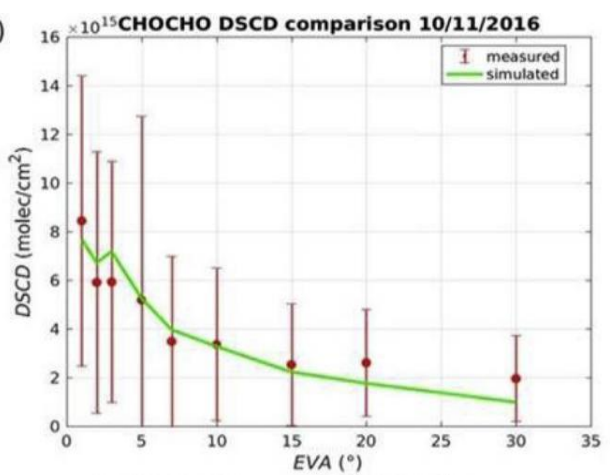

c)

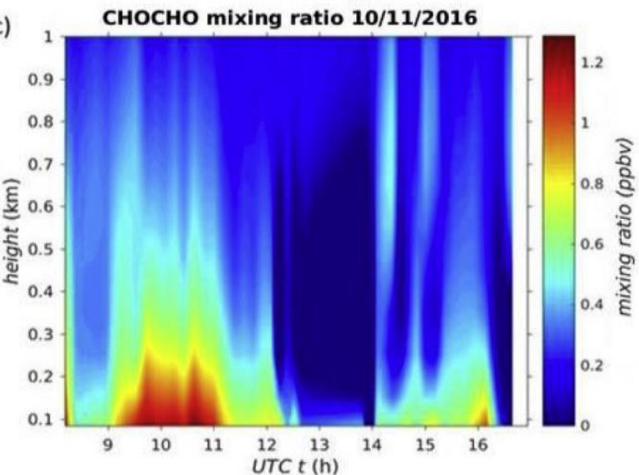

b)

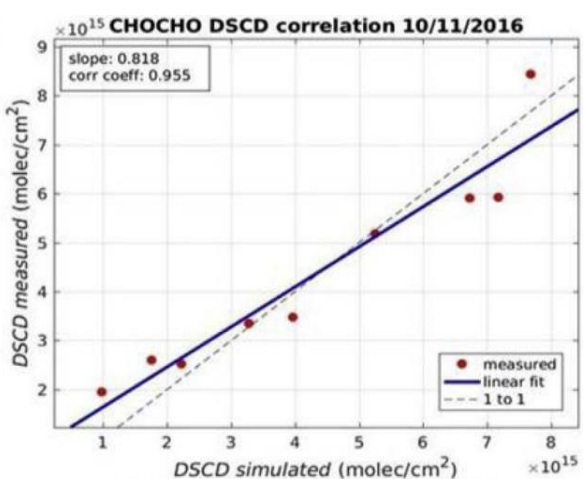

d)

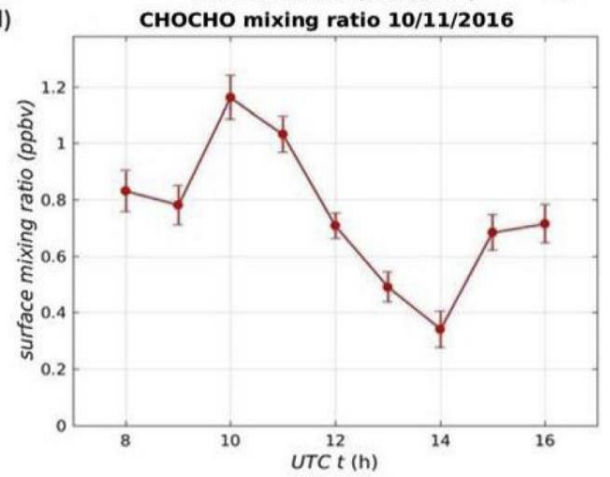

Figure 7.6. a) Measured and simulated $\mathrm{CHOCHO}$ DSCDs comparison, b) correlation between measured and simulated $\mathrm{CHOCHO}$ DSCDs, c) diurnal vertical profiles of $\mathrm{CHOCHO}$ mixing ratio d) $\mathrm{CHOCHO}$ surface mixing ratio. The measurements of these plots were all taken on November 10, 2016. 
Throughout the year, we had some measurement gaps caused by some saturation problems in the CCD detector, particularly in the VIS spectral region and around noon due to higher irradiance levels. Hence we show here one-day representative of the days when the spectra were not saturated at noon. Besides the saturation problems, few valid days remained from the afternoon glyoxal analysis after the quality check. Thus, we do not consider the number of noon and afternoon measurements to be statistically significant to assess their seasonality. We have therefore taken the early morning hours for the seasonal and long-term assessment of glyoxal levels. Monthly hourly-averaged glyoxal surface mixing ratios and VCDs at 09 UTC are illustrated in Figure 7.7. In Figure 7.7 we observe a decrease in the glyoxal levels throughout the year, with a maximum value of $2 \mathrm{ppbv}$ in February and a minimum value of $0.7 \mathrm{ppbv}$ in August. On the other hand, we found that the profiling algorithm for glyoxal was not sensitive enough to layers above $1 \mathrm{~km}$, thus the corresponding VCDs yielded larger uncertainties (and even two months, January and April, without reliable results for the entire column) than the surface mixing ratios. Our retrieved results of glyoxal VCDs are higher (in the order of twice as much) than other reported for urban environments using the DOAS technique (Sinreich et al., 2007) although there is a good agreement with the range reported in Khokhar et al., 2016. Our values fall within the typical range (0.04-4.1 ppbv) reported by the World Health Organization (Kielhorn et al., 2004) for urban environments. In addition, the lack of a strong temporal evolution compares to that observed for $\mathrm{HCHO}$ at the same time of day.

Although there are some previous studies of glyoxal from satellite measurements (Miller et al., 2014), we could not reliably compare our glyoxal VCDs with the glyoxal VCDs from the OMI satellite because at its passing time over Madrid (after noon time) we had some saturation problems with the CCD camera. 

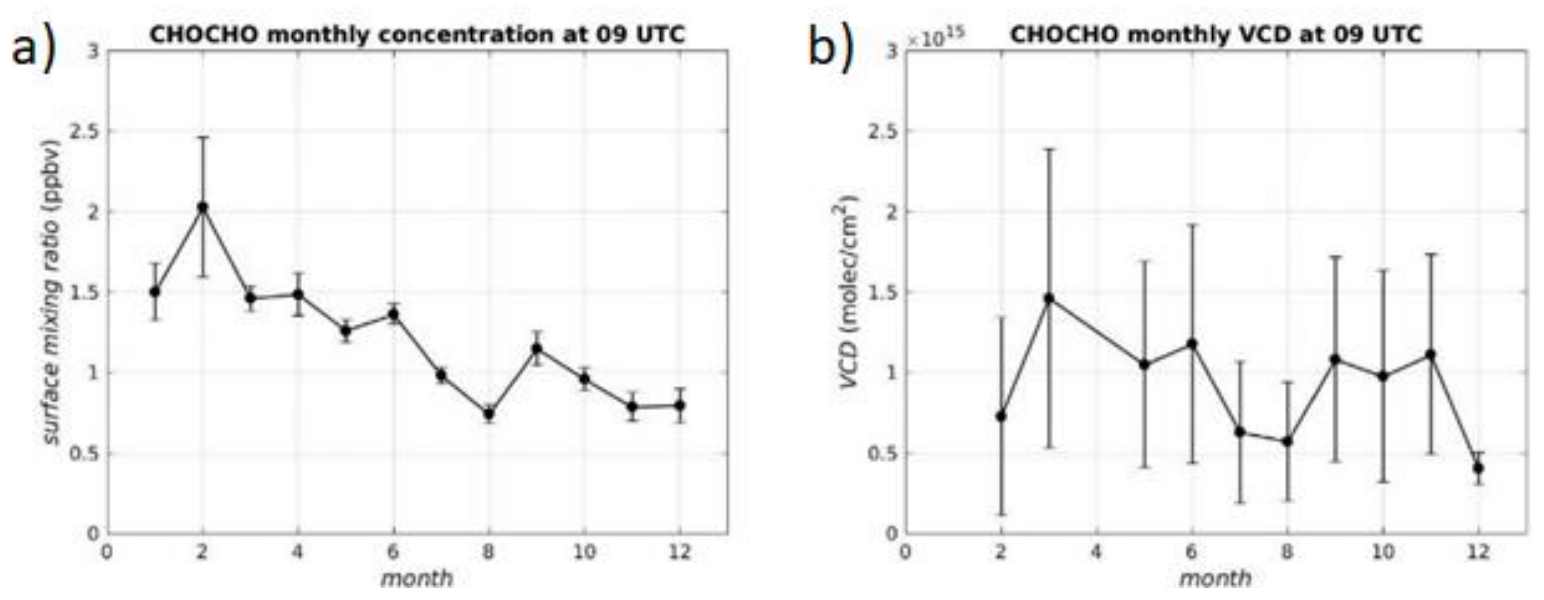

Figure 7.7. Glyoxal monthly variation at 09 UTC time of hourly averaged surface mixing ratios (a) and VCD (b).

\subsection{The glyoxal/formaldehyde ratio}

Previous studies have looked into the glyoxal/formaldehyde ratio as an indicator of the source of VOCs precursors, although there are discrepancies about how to interpret such ratio (Kaiser et al., 2015). However, one general idea derived from the existing literature is: high $R_{G F}$ seems to be correlated with biogenic emissions and low $R_{G F}$ with anthropogenic emissions. Unfortunately, in Madrid there are no measurements of isoprene or other VOCs that could help this study to further understand the interpretation of the observed $\mathrm{R}_{\mathrm{GF}}$ ratio.

For this reason, in order to relate the $\mathrm{R}_{\mathrm{GF}}$ to some information about the nature of emissions, we compared it with $\mathrm{NO}_{2}$ surface mixing ratios, which are associated with anthropogenic emissions (Vrekoussis et al., 2010). These $\mathrm{NO}_{2}$ values have been derived from the same MAXDOAS instrument and applying analogous calculations as for $\mathrm{HCHO}$ and glyoxal. On the other hand, the $\mathrm{R}_{\mathrm{GF}}$ has been retrieved taking the surface mixing ratios of glyoxal and formaldehyde. 


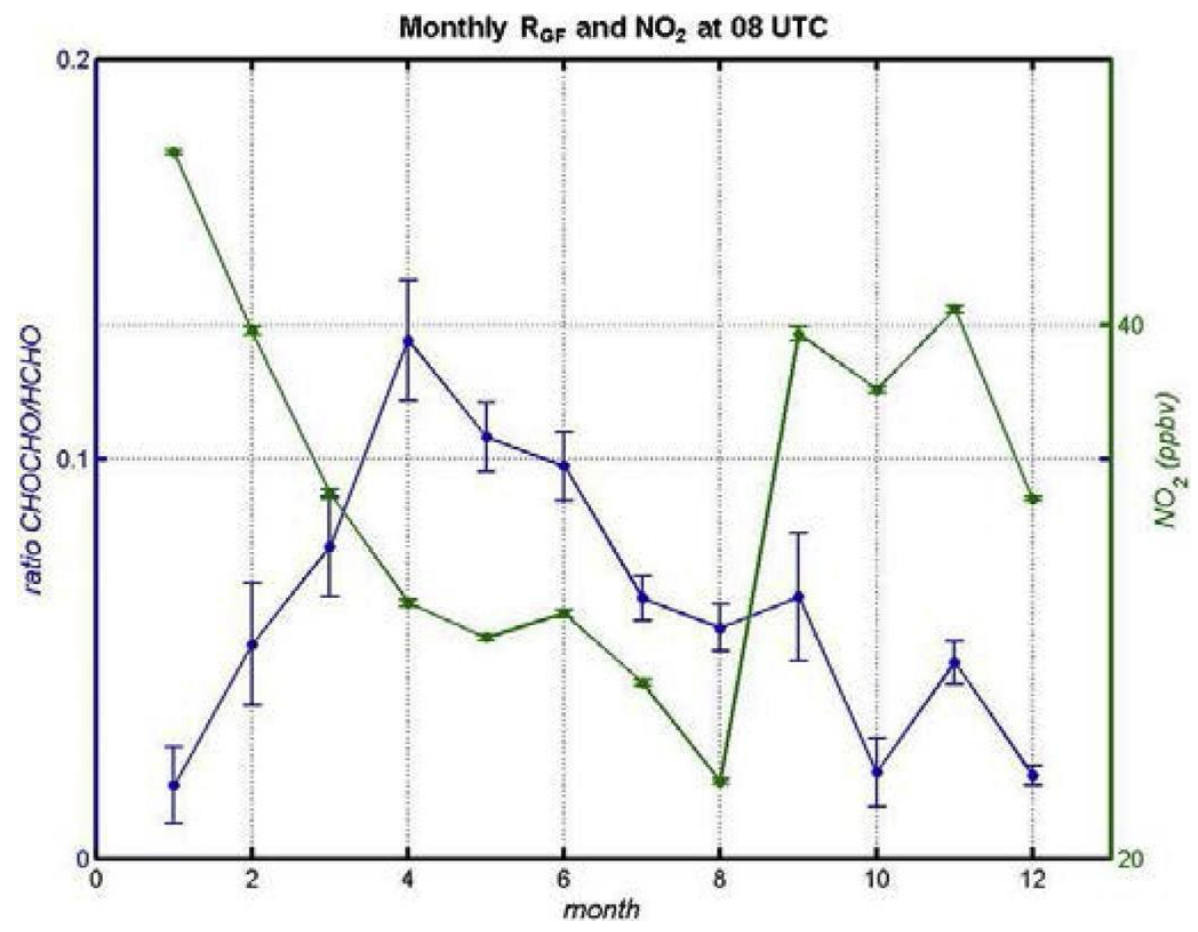

Figure 7.8. Monthly variation of $\mathrm{R}_{\mathrm{GF}}$ calculated from hourly averaged $\mathrm{CHOCHO}$ and $\mathrm{HCHO}$ surface mixing ratios at 08 UTC time (blue curve) and monthly variation of $\mathrm{NO}_{2}$ surface mixing ratio at 08 UTC time (green curve).

Figure 7.8 represents the monthly $\mathrm{R}_{\mathrm{GF}}$ ratios and $\mathrm{NO}_{2}$ levels hourly-averaged at 08 UTC. Both variables have been obtained taking the results of the first $100 \mathrm{~m}$ layer. Autumn and winter months have high $\mathrm{NO}_{2}$ mixing ratios, from $33 \mathrm{ppbv}$ to $46 \mathrm{ppbv}$, that are related to lower $\mathrm{R}_{\mathrm{GF}}$ values in these months (0.02-0.07). $\mathrm{NO}_{2}$ values range between 23 and $39 \mathrm{ppbv}$ in spring and summer, while the $\mathrm{R}_{\mathrm{GF}}$ varies within 0.07 and 0.13 during those seasons. Figure 7.8 also shows that $\mathrm{R}_{\mathrm{GF}}$ has a trend which is anticorrelated with the $\mathrm{NO}_{2}$ values reinforcing the scenario of more prevalent anthropogenic than biogenic emissions when the $\mathrm{R}_{\mathrm{GF}}$ levels are lower. 


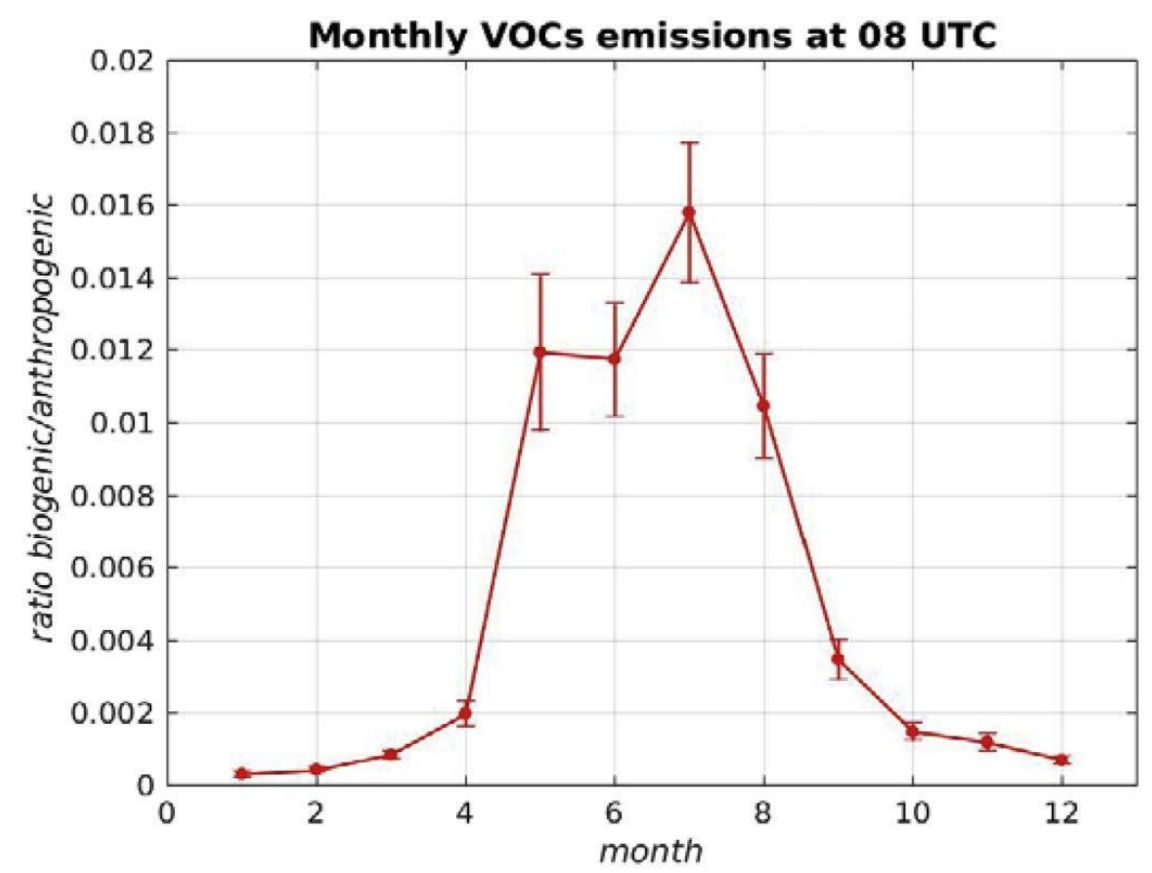

Figure 7.9. Monthly variation of the ratio between biogenic and anthropogenic VOCs emissions in Madrid for the year 2015. The ratio was taken using hourly averaged values of the emissions inventories at 08 UTC.

To further support this idea, in this study we have also included VOCs emissions inventories within the city centre of Madrid. Anthropogenic emissions have been estimated using the SMOKE model (Sparse Matrix Operator Kernel Emissions Modeling System, UNC Carolina Environmental Program, 2005, described in Borge et al., 2018) and biogenic emissions have been evaluated by means of the MEGANv2.10 model (Borge et al., 2008 and Gunther et al., 2012).

Although these emissions inventories could only provide values for Madrid as recent as 2015, we still took the year 2015 assuming that the emissions should be comparable to those during 2016 (when our MAX-DOAS measurements were carried out). Figure 7.9 shows the monthly hourly-averaged ratio between biogenic and anthropogenic VOCs emissions in Madrid at 08 UTC time. Note that in summer the relative contribution of biogenic emissions is higher than in winter. This modelled VOCs emissions ratio has a comparable trend to the $\mathrm{R}_{\mathrm{GF}}$ curve shown in Figure 7.8 in which summer and spring months 
have higher $\mathrm{R}_{\mathrm{GF}}$ than autumn and winter. This supports the notion of relatively larger biogenic emissions in spring and summer inferred by the measured $\mathrm{R}_{\mathrm{GF}}$.

Note that this work only considers the variation between different months in a specific hour. $R_{G F}$ values fall roughly between 0.02 and 0.13 , which are in good agreement with ranges previously reported in other urban environments (Grosjean et al., 1990 in Sao Paulo; Grosjean et al., 1996 in Los Angeles; Ho et al., 2002b in Hong Kong). Regarding the monthly evolution, Liu et al., 2006 provide higher values (between 0.15 in winter and 0.35 in summer) but with a similar trend to that obtained in this work (higher values during spring and summer) although taking daily averages. Nevertheless, more combined $\mathrm{HCHO}$, $\mathrm{CHOCHO}$ and other VOCs measurements in different areas are needed to get a better knowledge of the meaning of the $\mathrm{R}_{\mathrm{GF}}$ ratio. 


\section{DEVELOPMENT OF A MAXDOAS-}

\section{D INSTRUMENT}

The development, set-up and analysis of the MAXDOAS-2D instrument is described in this chapter. In Section 8.1 a brief introduction to the measurements is made. Then, the experimental set-up and analysis methods are explained. Moreover, in Section 8.4 some inversion algorithm details and the related parameters are treated. The two-dimensional maps of trace gases vertical profiles are new in Madrid; this is the first time that this type of maps are presented in this city (Section 8.5) as well as their horizontal distribution (Section 8.6). To finish this section, a comparison between both MAXDOAS-2D measurements and the in situ monitors of the City Council of Madrid is performed. The content in this chapter is under review in Garcia-Nieto et al., 2020.

\subsection{Introduction}

In an effort to control and reduce high pollution events, the local government has enforced some traffic restriction measures (Izquierdo et al., 2020) and has set up several in-situ air quality monitoring stations over the metropolitan area of the city. These in situ instruments -as of today- cannot measure some important trace gases present in the atmosphere and their values are only representative of the immediate surrounding of the instruments and at surface level. There is therefore a need for mesoscale analysis (both in horizontal and vertical) of urban air pollution that could complement the in-situ measurements. In addition to routinely monitored, regulated species such as $\mathrm{NO}_{2}$ and $\mathrm{O}_{3}$. Over the past few years, we have reported trace gas measurements in Madrid using the MAXDOAS technique (Wang et al., 2016; Garcia-Nieto et al., 2018; Benavent et al., 2019) 
as well as pollutants trend analysis and chemical transport modelling (Borge et al., 2018; Cuevas et al., 2018; Saiz-Lopez et al., 2017).

During the course of this thesis, a new two-dimensional MAXDOAS instrument has been built, tested and set up to take continuous measurements in Madrid. This instrument represents a follow-up development to our previous one-dimensional instrument (MAXDOAS-1D, see Garcia-Nieto et al., 2018) that incorporates the capability of moving in the azimuthal dimension, therefore allowing the collection of spectra pointing at any desired angular direction. This additional capability allows the measurement of both the horizontal and vertical trace gas (e.g. $\mathrm{NO}_{2}$ ) distribution throughout the city and in turn the generation of two-dimensional maps of trace gas composition. Several works using twodimensional MAXDOAS instruments have been carried out in recent years (e.g. Ortega et al., 2015, Schreier et al., 2019, Dimitropoulou et al., 2020). These studies were mostly focused on mapping the $\mathrm{NO}_{2}$ distribution in urban environments and assessing its role for air quality monitoring.

Here, two months of MAXDOAS-2D measurements of scattered sunlight spectra are presented. The measurements were taken from May 6, 2019 to July 5, 2019, with focus on the evaluation of $\mathrm{NO}_{2}$ vertical concentration profiles and the characterization of horizontal light paths lengths. We will also provide the retrieval of HONO as an example of the potential of the MAXDOAS-2D measurements. This represents the first two-dimensional MAXDOAS measurements in Madrid. An assessment of the relation between the MAXDOAS analysis and the in situ instruments in the city was carried out

\subsection{Experimental}

For these two-dimensional measurements we used the MAXDOAS-2D described in Section 5.3. Using the DOAS technique, the absorptions of the molecular oxygen dimer and $\mathrm{NO}_{2}$ were measured for the entire campaign and for two spectral windows: 352-387 nm (UV region) and 438-487 $\mathrm{nm}$ (VIS region). The analysis settings applied for the UV and VIS 
regions are summarized in Tables 8.1 and 8.2, respectively. These configurations follow those used in Wagner et al., 2018.

Table 8.1. DOAS fit settings for the retrieval of $\mathrm{O}_{4}$ and $\mathrm{NO}_{2}$ (UV).

\begin{tabular}{|c|c|}
\hline Parameter & Value \\
\hline Fitting window & $352-387 \mathrm{~nm}$ \\
\hline Wavelength calibration & $\begin{array}{l}\text { Based on reference solar atlas (Chance and } \\
\text { Kurucz, 2010) }\end{array}$ \\
\hline Zenith reference & Scan \\
\hline Polynomial Order & 5 \\
\hline Intensity Offset & Order 2 \\
\hline Shift & $\begin{array}{l}\text { The measured spectra and the Ring cross } \\
\text { sections were allowed to shift and stretch } \\
\text { (order } 1 \text { ) in wavelength. }\end{array}$ \\
\hline Molecule & Cross section \\
\hline $\mathrm{O}_{4}$ & $293 \mathrm{~K}$ (Thalman and Volkamer, 2013) \\
\hline $\mathrm{NO}_{2}$ & 298 K (Vandaele et al., 1998) \\
\hline $\mathrm{O}_{3} \mathrm{a}$ & 223 K (Serdyuchenko et al., 2014) \\
\hline $\mathrm{O}_{3} \mathrm{~b}$ & 293 K (Serdyuchenko et al., 2014) \\
\hline $\mathrm{HCHO}$ & 297 K (Meller and Moortgat, 2000) \\
\hline HONO & 296 K (Stutz et al., 2000) \\
\hline Ring a & Calculated by QDOAS \\
\hline
\end{tabular}


Ring b

Ring a spectrum multiplied by $\lambda^{-4}$

Table 8.2. DOAS fit settings for the retrieval of $\mathrm{O}_{4}$ and $\mathrm{NO}_{2}$ (VIS).

\begin{tabular}{|c|c|}
\hline Parameter & Value \\
\hline Fitting window & $438-487 \mathrm{~nm}$ \\
\hline Wavelength calibration & $\begin{array}{l}\text { Based on reference solar atlas (Chance and } \\
\text { Kurucz, 2010) }\end{array}$ \\
\hline Zenith reference & Scan \\
\hline Polynomial order & 5 \\
\hline Intensity offset & Order 2 \\
\hline Shift & $\begin{array}{l}\text { The measured spectra and the Ring cross } \\
\text { sections were allowed to shift and stretch } \\
\text { (order 1) in wavelength. }\end{array}$ \\
\hline Molecule & Cross section \\
\hline $\mathrm{O}_{4}$ & $293 \mathrm{~K}$ (Thalman and Volkamer, 2013) \\
\hline $\mathrm{NO}_{2}$ & 298 K (Vandaele et al., 1998) \\
\hline $\mathrm{O}_{3} \mathrm{a}$ & $223 \mathrm{~K}$ (Serdyuchenko et al., 2014) \\
\hline $\mathrm{O}_{3} \mathrm{~b}$ & $223 \mathrm{~K}$ (Serdyuchenko et al., 2014) \\
\hline $\mathrm{H}_{2} \mathrm{O}$ & 296 K (Rothman et al., 2010) \\
\hline Glyoxal & 296 K (Volkamer et al., 2005) \\
\hline Ring a & Calculated by QDOAS \\
\hline Ring $b$ & Ring a spectrum multiplied by $\lambda^{-4}$ \\
\hline
\end{tabular}




\subsection{Analysis methods}

\subsubsection{Spectral detection}

The selected differential absorption cross sections -along with the spectral window and fitting parameters- have been included in Tables 8.1 and 8.2. Figure 8.1 shows examples of the spectral detection of $\mathrm{O}_{4}$ and $\mathrm{NO}_{2}$ for both the UV and VIS regions.
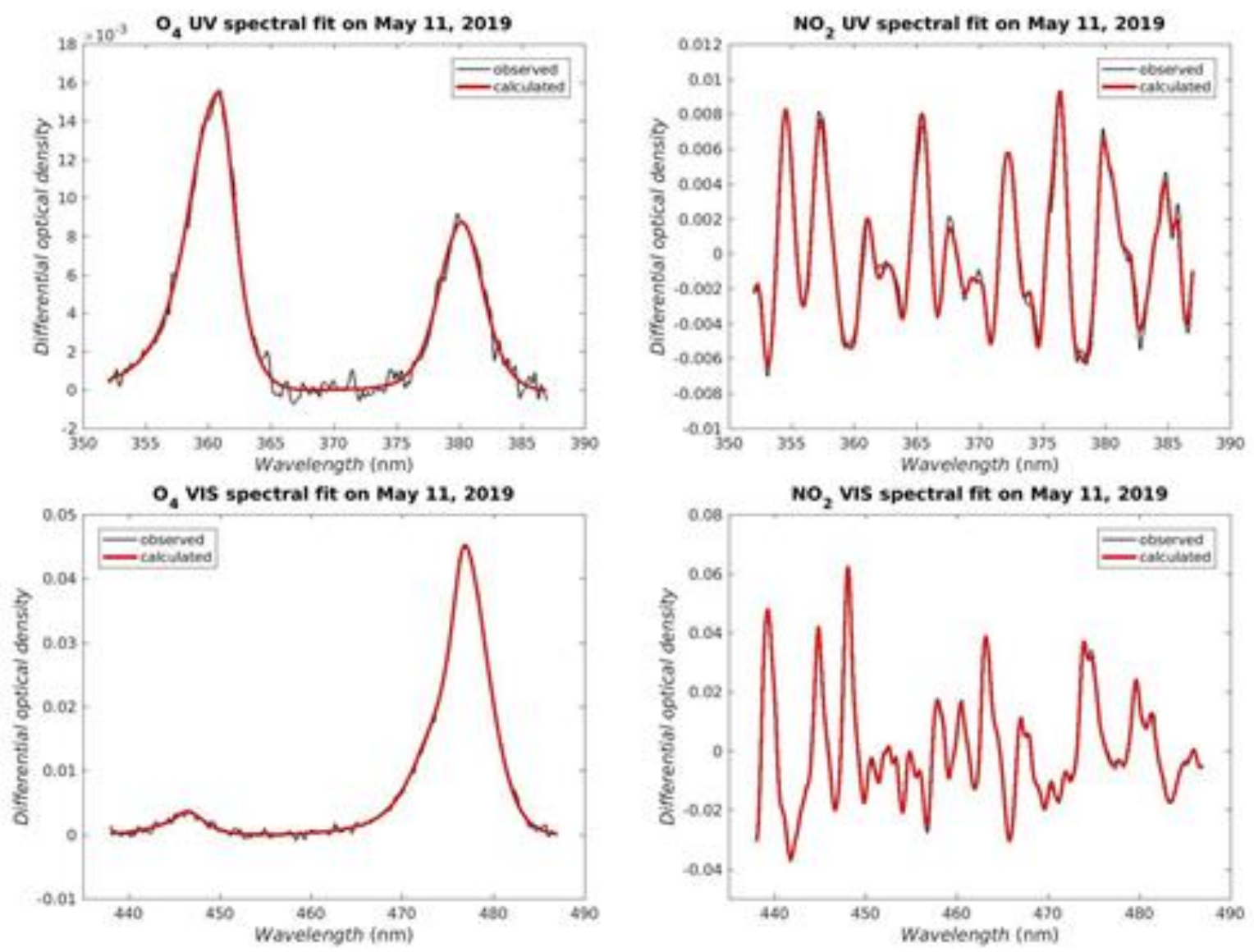

Figure 8.1. Spectral detection of $\mathrm{O}_{4}(\mathrm{a})$ and $(\mathrm{c})$ and $\mathrm{NO}_{2}(\mathrm{~b})$ and $(\mathrm{d})$, red lines represent the calculated optical densities and black lines are the measured optical densities.

\subsubsection{Inversion algorithm and vertical profiles}

For the inversion algorithm we used similar ideas to those explained in Section 6.3. But first of all, we applied the cloud screening and quality filtering criteria detailed in 
Chapter 4 for the retrieved DSCDs. Then, we chose typical values for urban environments: surface albedo of 0.07 , correlation length of $0.4 \mathrm{~km}$ and an a priori covariance factor of 1 (see Hendrick et al., 2014). However, the most important set of parameters are the atmospheric profiles such as the air number density vertical profile since it is directly related to the number of $\mathrm{O}_{4}$ absorptions, and therefore to the $\mathrm{O}_{4}$ DSCDs. Hence the relative differences, particularly for lower VEAs, between the measured and simulated $\mathrm{O}_{4} \mathrm{DSCDs}$ are usually assigned to aerosol extinction. Note however, that uncertainties in the air number density profiles -arising from uncertainties in the values or shape of the temperature and pressure profiles- could also explain such differences (Figure 8.2).
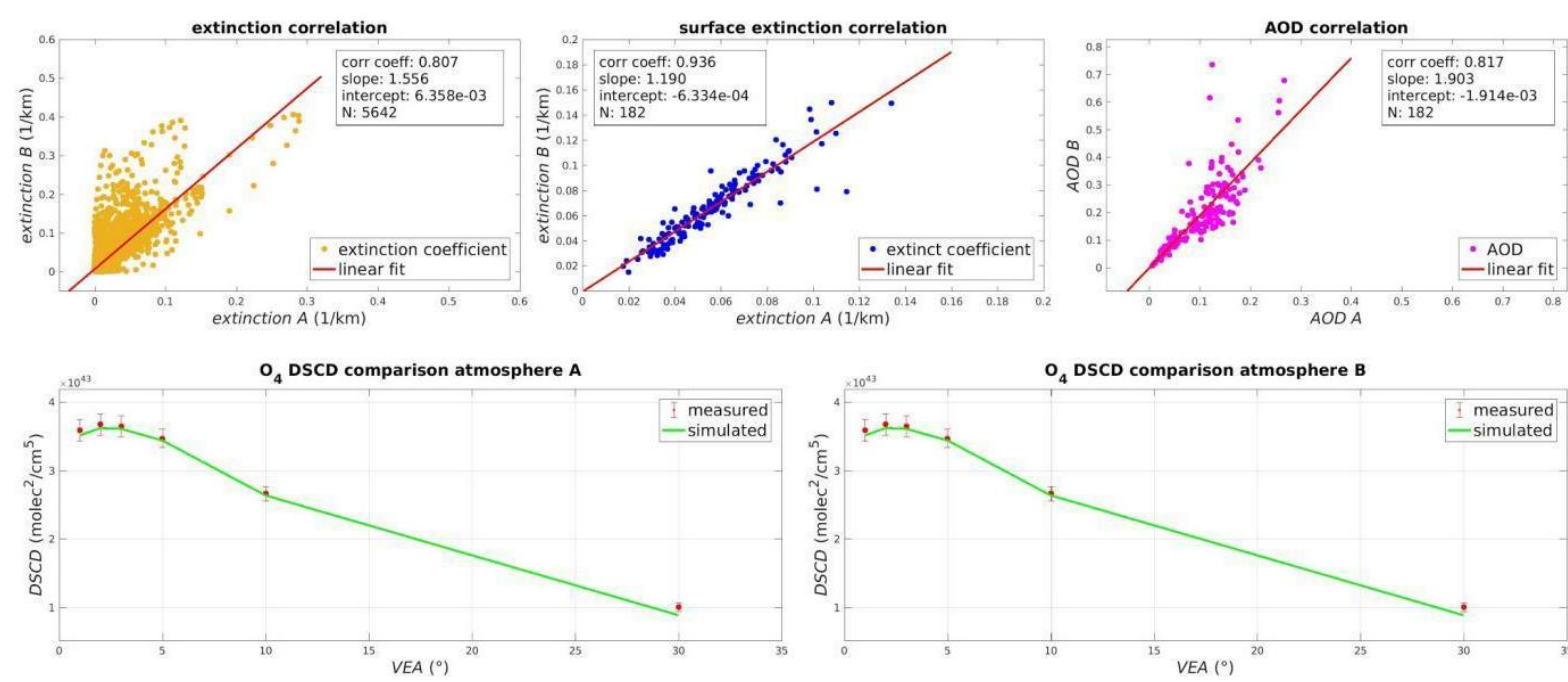

Figure 8.2. Comparison of retrieved aerosols using two different atmospheric profiles: the U.S. Standard Atmosphere (atmosphere A) and the U.S. Standard Atmosphere adapted to the altitude above sea level of Madrid (atmosphere B).

Here we compare the simulation of $\mathrm{O}_{4}$ DSCDs using two different sets of atmospheric profiles: i) the U.S. Standard Atmosphere, and ii) the same profile but interpolating the pressure profile to the height of Madrid above sea level (mean value of $667 \mathrm{~m}$ ). This means that the temperature profile is assumed to be the same but the pressure profile is shifted less than $10 \%$, so there are no major variations within the 
profiles. The lower row in Figure 8.2 shows that both atmospheric profiles result in almost the same set of simulated $\mathrm{O}_{4}$ DSCDs, however the aerosol extinction coefficients differ significantly (although less for the surface layer coefficients), and consequently, the AOD also varies. From this we infer that:

i) the retrieval is mainly driven by the measured DSCDs, which leaves a relatively low weight for the chosen atmospheric profiles (pressure and temperature). Therefore, we can achieve very consistent, solid correlations between the measured and simulated $\mathrm{O}_{4}$ DSCDs.

ii) we cannot reliably assign the extinction coefficients at each layer to particulate aerosol (especially for atmospheric layers above the surface layer), but rather consider them as irradiance extinction coefficients.

Furthermore, we have assessed the impact of the pressure and temperature profiles choice on the trace gas retrieval. As can be noted in Figure 8.3, there is no significant effect coming from this choice on the simulated $\mathrm{NO}_{2}$ DSCDs. These are basically the same (and with very good agreement with the measured DSCDs), as well as the derived concentration coefficients and their integration (VCD). Consequently, we have decided to use the U.S. Standard Atmosphere profiles for the $\mathrm{NO}_{2}$ retrievals in this study. 

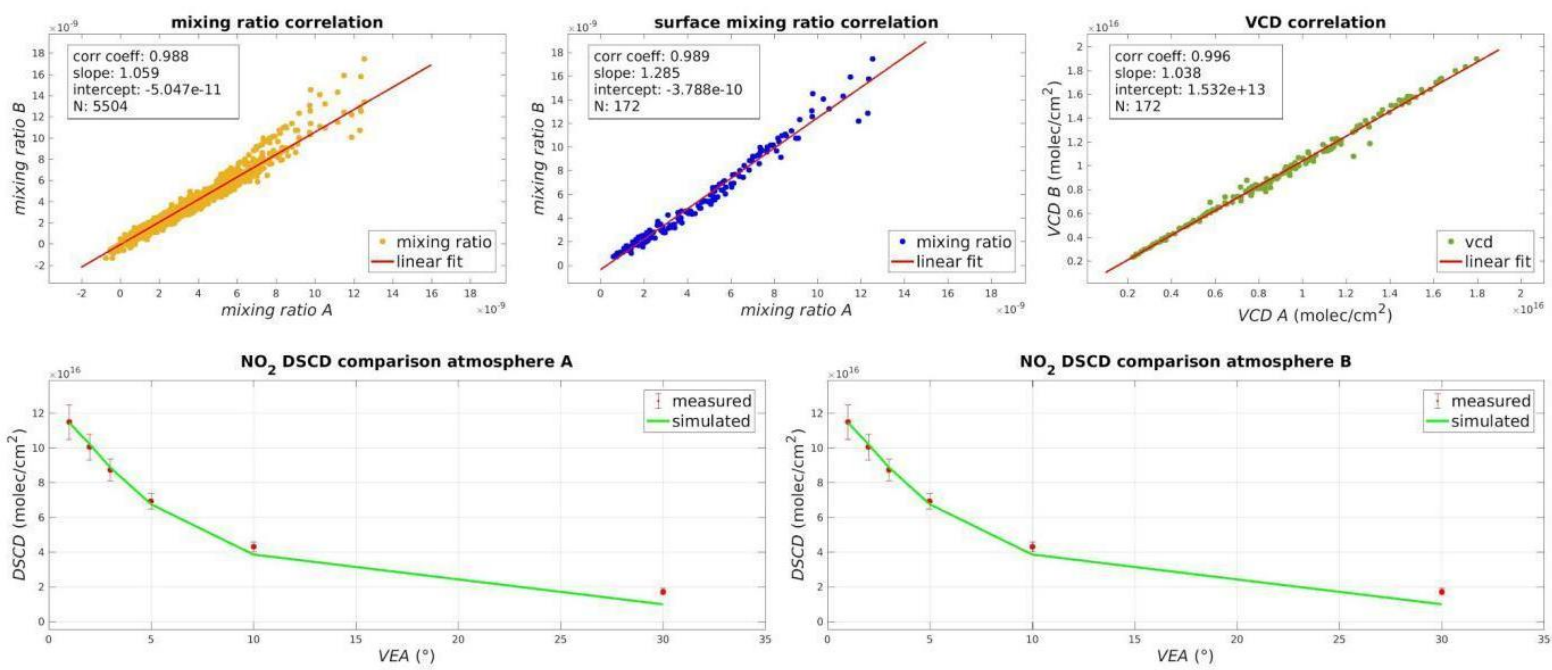

Figure 8.3. $\mathrm{NO}_{2}$ retrieval comparison using two different atmospheric profiles: the US Standard Atmosphere (atmosphere A) and the US Standard Atmosphere adapted to the altitude above sea level of Madrid (atmosphere B).

However, we also evaluated if a similar behavior can be expected for larger variations in the pressure and temperature profiles. We first obtained the average surface temperature and pressure values for the duration of the campaign (May-July, 2019). With the inclusion of these values in the retrieval, we found that, within the first $10 \mathrm{~km}$ height, the RMS of the relative variations with respect to the standard atmosphere were about 8 $\%$. Although it is a small change, it is indeed not negligible. Nonetheless, when evaluating light paths, the relative changes were below $2 \%$. Consequently, we have decided to use the U.S. Standard Atmosphere profiles for the $\mathrm{NO}_{2}$ retrievals in this study.

But apart from that, we also assessed the influence of several other inversion parameters for the purpose of seeing if there was a significant impact of any of them. Here we show a couple more comparisons. The first one corresponds to the a priori extinction profile: we checked the influence of two different a priori extinction profiles (with integrated extinction values of 0.05 and 0.1 ). Figure 8.4 includes this comparison. 

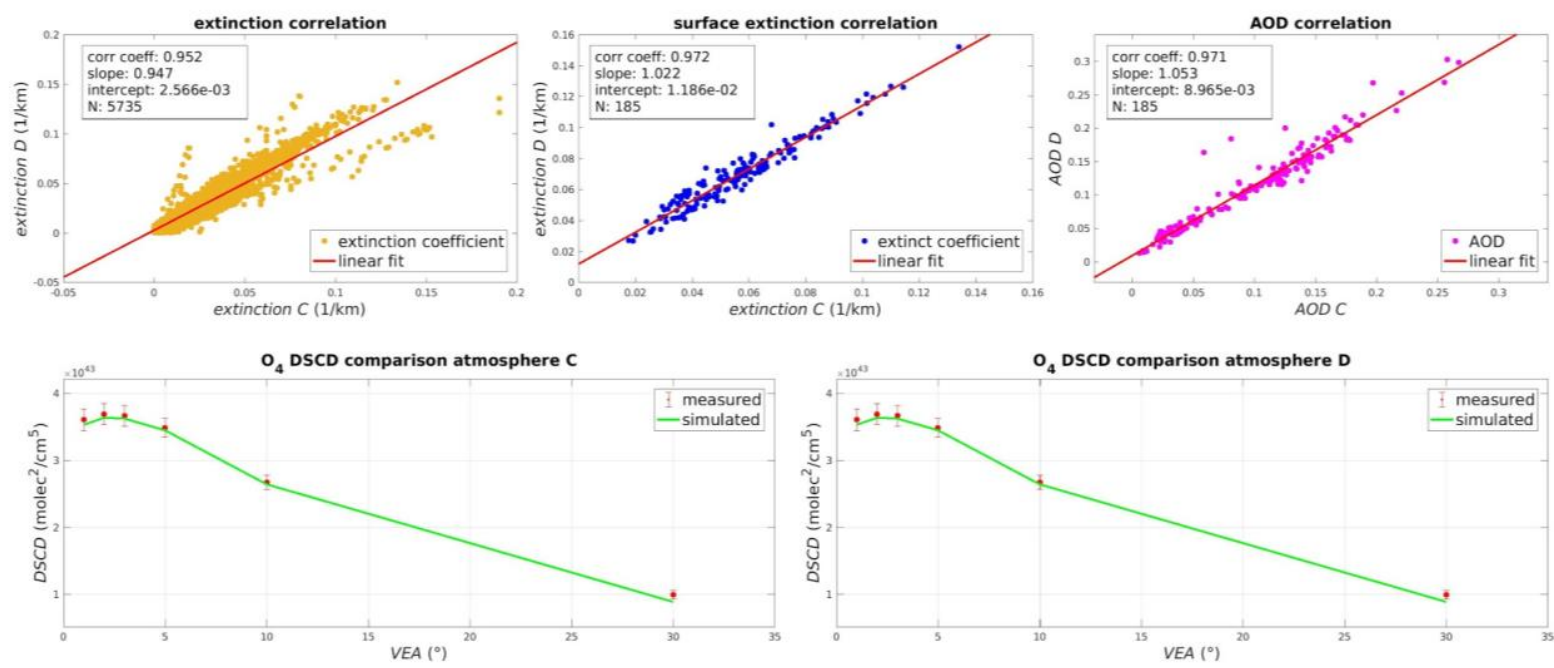

Figure 8.4. Comparison of the simulated $\mathrm{O}_{4} \mathrm{DSCDs}$ and the retrieved extinction coefficients using two different a priori column-integrated extinctions: a total extinction of 0.05 (atmosphere C) and a total extinction of 0.1 (atmosphere D).

We observe that there is no significant impact when using different a priori profiles for the extinction coefficients. Again very solid correlations are found (correlation coefficients higher than 0.95 and slopes close to 1 for every magnitude), and the main conclusion remains: the principal driver of the extinction retrieval is the measured $\mathrm{O}_{4}$ DSCDs vector.

The second comparison concerns the surface albedo coefficient: now two different surface albedo coefficients ( 0.07 and 0.7 ) are explored. Figure 8.5 shows the comparison. Once more we obtain quite similar simulated $\mathrm{O}_{4} \mathrm{DSCDs}$, but the extinction coefficients -and thus their integrated AOD- differ, although offering robust correlations (with coefficients higher than 0.83 for every magnitude). 

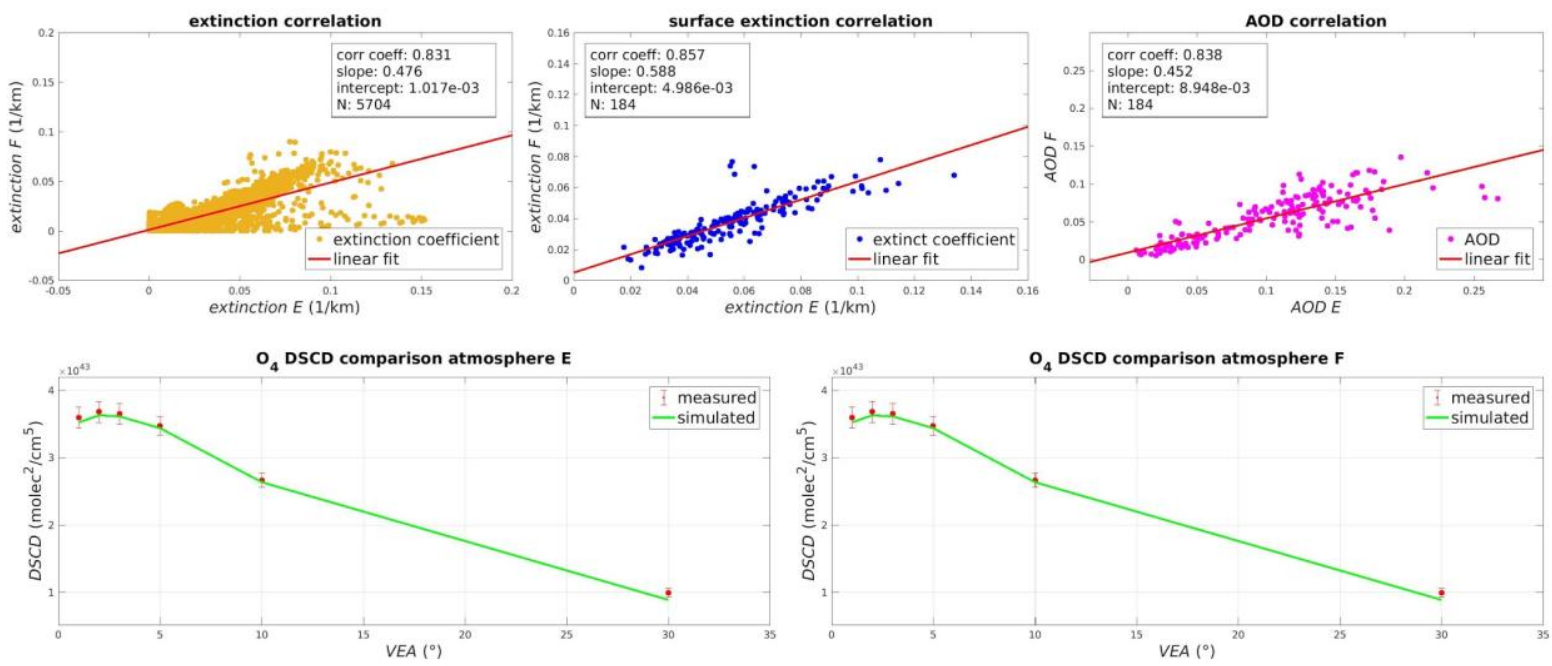

Figure 8.5. Comparison of the simulated $\mathrm{O}_{4}$ DSCDs and the retrieved extinction coefficients using two different surface albedo coefficients: 0.07 (atmosphere E) and 0.7 (atmosphere F).

These last two comparisons further contribute to the conclusions reached in the discussion after Figure 8.2.

Table 8.3 summarizes the average uncertainties (using one standard deviation for each component) of the retrieval, along with their relative contributions, for the ground layer (0-200 $\mathrm{m}$ height). The mean, overall uncertainty for $\mathrm{NO}_{2}$ in both spectral regions is in the order of $10 \%$. 
Table 8.3. Summary of average uncertainties of the retrieval in both spectral regions.

\begin{tabular}{|c|c|c|}
\hline Variable \Trace gas & $\mathbf{N O}_{2}$ UV (\%) & NO $_{2}$ VIS (\%) \\
\hline Irradiance Extinction & 7.7 & 5.1 \\
\hline DSCD & 4.8 & 3.2 \\
\hline Surface Mixing Ratio & 5 & 8.7 \\
\hline Total & 10 & 11 \\
\hline
\end{tabular}

\subsubsection{Estimation of $\mathrm{NO}_{2}$ gradients}

Making use of the different paths that photons travel through the atmosphere for different wavelengths, we can estimate the horizontal distribution of $\mathrm{NO}_{2}$. We use the estimated horizontal light paths at two wavelengths, $360.8 \mathrm{~nm}$ and $477 \mathrm{~nm}$, for the surface layer (0-200 m height). The different light paths at 360.8 and $477 \mathrm{~nm}$ provide information about the horizontal distribution of $\mathrm{NO}_{2}$ mixing ratios within the surface layer. In order to evaluate these horizontal paths, we have used our own codes that implement the RTM equations based on previous pioneering work (Solomon et al., 1987). These equations yield a vector of scattering events along with their respective probabilities. If we take a VEA of $0^{\circ}$ (i.e. horizontal viewing), then the scalar product of such vectors produces the length of the horizontal light path. We computed this for every MAXDOAS cycle and for both wavelengths, yielding typical -representative- horizontal distances of about 8-10 km for the UV (at $360.8 \mathrm{~nm}$ ) and between 15-20 km for the VIS window (at $477 \mathrm{~nm}$ ). The next step follows the estimation of $\mathrm{NO}_{2}$ gradients approach proposed by Ortega et al. 2015 (the strong dependence of scattering with wavelength means that shorter wavelengths result in shorter light paths). Therefore, we first assign the UV (i.e. $360.8 \mathrm{~nm}$ ) mixing ratios $\left(m r_{u v}\right)$ and their expected horizontal paths $\left(d_{u v}\right)$ to the first peel $\left(m r_{A}\right.$, meaning zone A). Then the second peel (zone $B, m r_{B}$ ) can be derived as: 


$$
m r_{B}=\frac{m r_{v i s} \times d_{v i s}-m r_{u v} \times d_{u v}}{d_{v i s}}
$$

Thus we can derive mixing ratio values $\left(m r_{a}\right.$ and $\left.m r_{b}\right)$ representative of two different horizontal distances for each VAA.

\subsection{Results and discussion}

\subsection{1 $\mathrm{O}_{4}$ and $\mathrm{NO}_{2}$ DSCDs assessment}

Once the vertical profiles were retrieved using the RTM explained in Chapter 4, an overall comparison between the measured and simulated DSCDs was carried out. The fit between the measured and simulated DSCDs show correlation coefficients $\left(r^{2}\right)$ as well as slopes very close to 1 for both $\mathrm{O}_{4}$ and $\mathrm{NO}_{2}$ in the UV and VIS regions (Figure 8.6). That signals a solid, robust analysis.
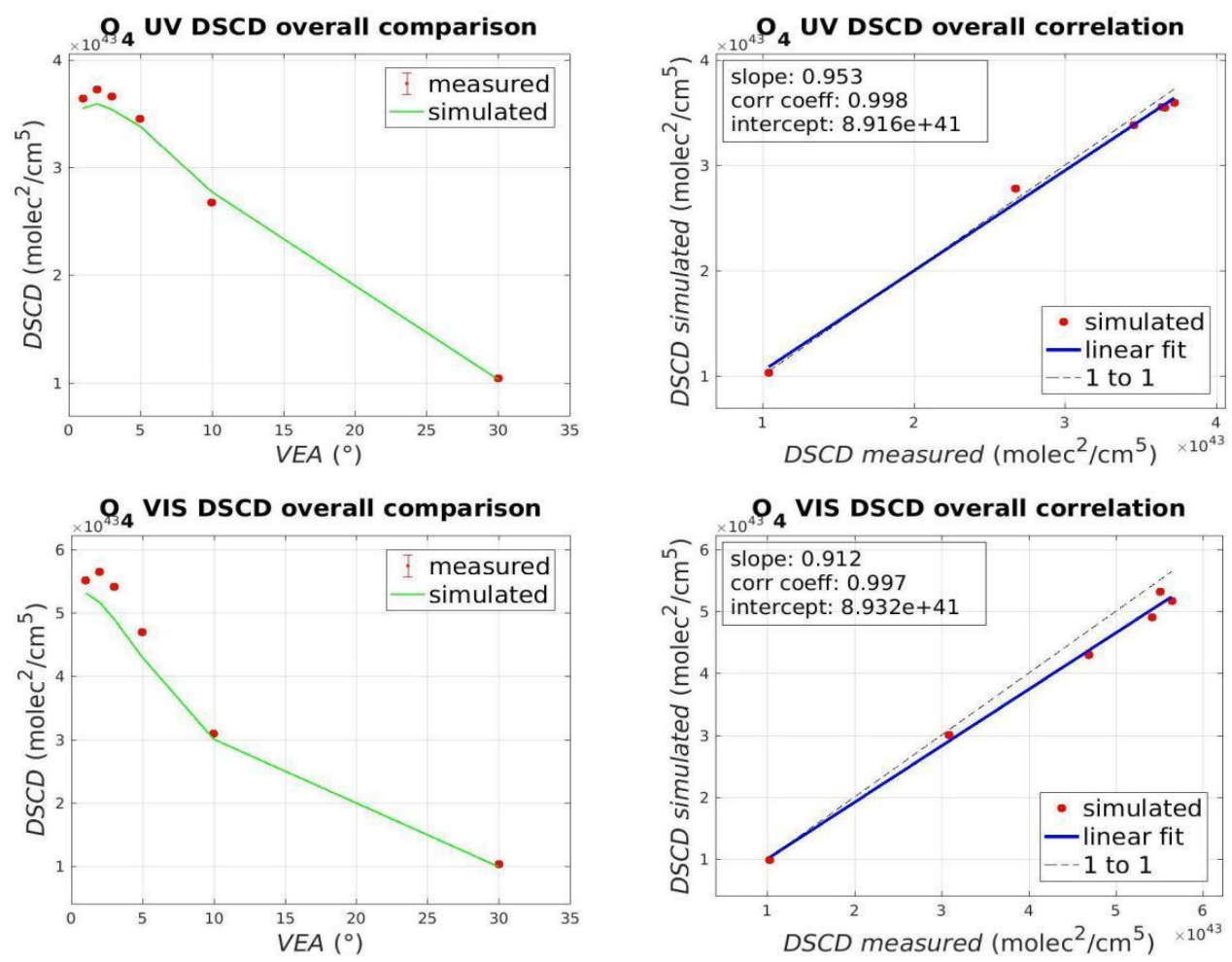

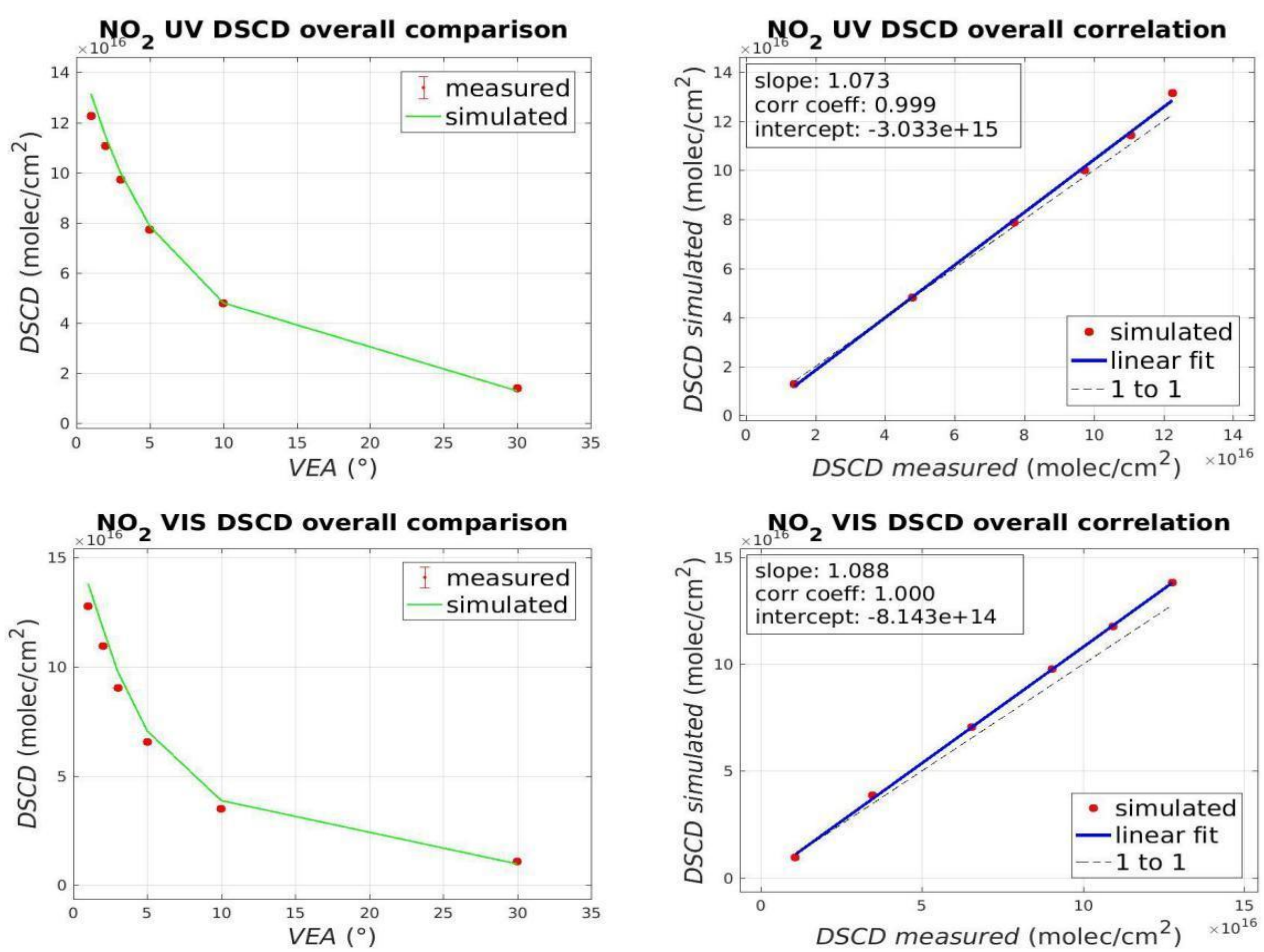

Figure 8.6. Comparison between simulated and measured DSCDs of $\mathrm{O}_{4}$ and $\mathrm{NO}_{2}$ for the entire campaign.

\subsubsection{Two-dimensional maps}

We now combine the VAA and height for each azimuthal cycle of the MAXDOAS-2D to generate a two-dimensional concentration map. Figure 8.7 shows an example of the $\mathrm{O}_{4}$ retrieval in the UV for a given azimuthal cycle. In addition to the profiles, Figure 8.7 also shows the comparison and correlation of measured and simulated DSCDs for that azimuthal cycle, along with the evolution of the retrieved AOD. The AOD varies between 0.05 and 0.18 within this azimuthal cycle (Figure 8.7, upper panel). The contour plot shows the irradiance extinction coefficient profiles with maximum values of $0.14 \mathrm{~km}^{-1}$ (near the ground and at around $40^{\circ}$ VAA) associated with aerosol extinction. Note the enhanced extinction at about $2 \mathrm{~km}$ height pointing at $50^{\circ} \mathrm{VAA}$. This could be due to particulate matter emitted by traffic (there is a main road at that location) or it could be associated with a residual aerosol layer remaining from the preceding night (Carnerero et al., 2019). Further 
research is needed to better establish the vertical distribution of aerosols in Madrid, and their diurnal evolution.
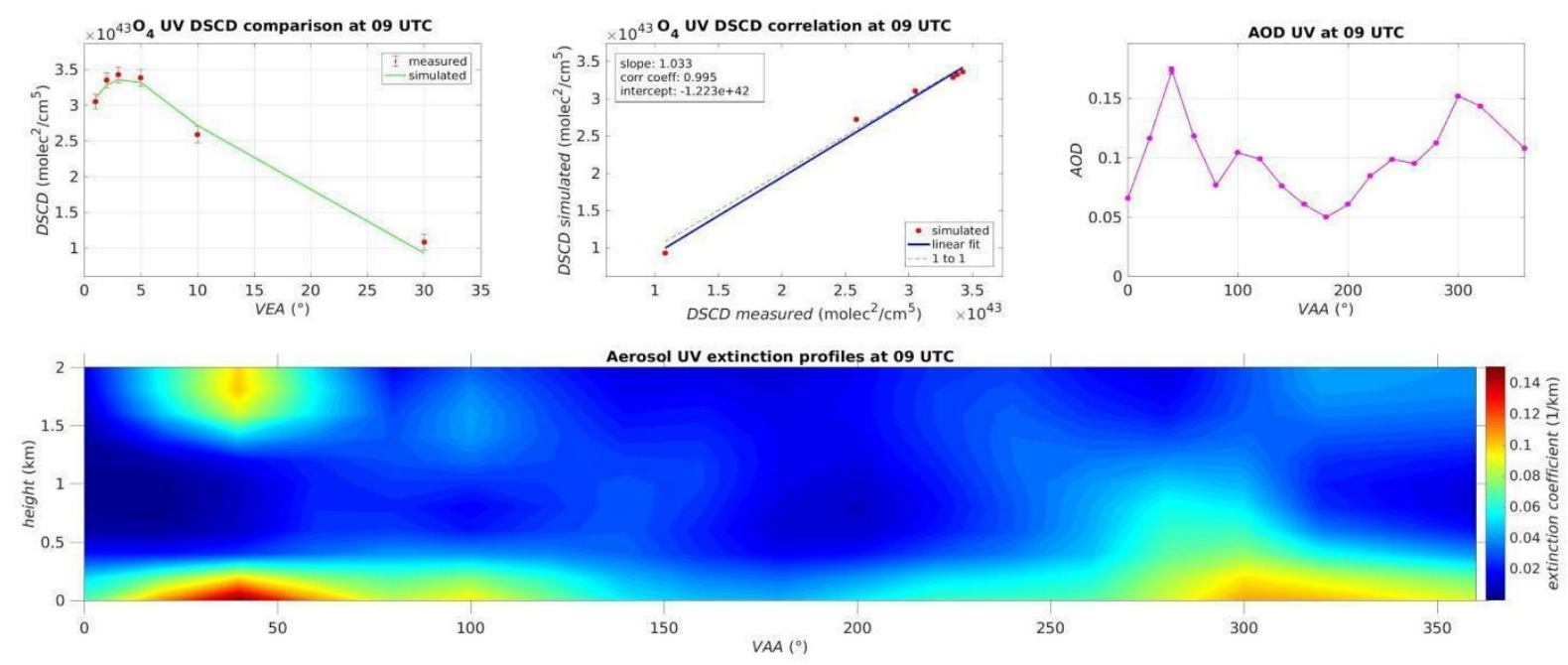

Figure 8.7. Example of $\mathrm{O}_{4}$ and AOD retrievals in the UV region at 9 UTC on May 11, 2019. These contour plots are smoothed from adjacent VAA data points separated by $20^{\circ}$ in order to estimate the azimuthal distribution of the irradiance extinction coefficients over Madrid.

Figure 8.8 presents a two-dimensional representation of $\mathrm{NO}_{2}$ on May 11, 2019 at two different hours ( 6 UTC and 12 UTC, respectively). Both contour plots show maximum $\mathrm{NO}_{2}$ values of $12 \mathrm{ppbv}$ at 6 UTC and $8 \mathrm{ppbv}$ at 12 UTC, when the instrument is pointing south (i.e. VAA of $180^{\circ}$ ). We chose to show this day as an example since it was a clear sky day and offered $\mathrm{NO}_{2}$ mixing ratios that were representative of the entire period of measurements. These values are found in the layer near the ground and are in good agreement with our previous MAXDOAS observations in Madrid (Garcia-Nieto et al., 2018). The retrieved azimuthal distribution of $\mathrm{NO}_{2}$ agrees with previous reports that show higher pollution levels in the southern section of Madrid (Picornell et al., 2019). $\mathrm{NO}_{2}$ VCDs range from $5 \times 10^{15}$ molecules $\mathrm{cm}^{-2}$ (at 12 UTC and pointing at $300^{\circ}$ VAA) up to $15 \times 10^{15}$ molecules $\mathrm{cm}^{2}$ (at 12 UTC and pointing at $200^{\circ}$ VAA), with an average value of $1 \times 10^{16}$ molecules $\mathrm{cm}^{-2}$. Although there can be different $\mathrm{NO}_{x}$ emission rates at both times of the day ( 6 and 12 UTC), the increase in the boundary layer height during the day could explain the similar values of 
VCDs at both hours but generally lower surface mixing ratios at $12 \mathrm{UTC}$. Note that $\mathrm{NO}_{2}$ is efficiently mixed within the boundary layer as it develops during the day (i.e. boundary layer height usually lags the solar zenith angle) (Figure 8.8) (de la Paz et al., 2016).
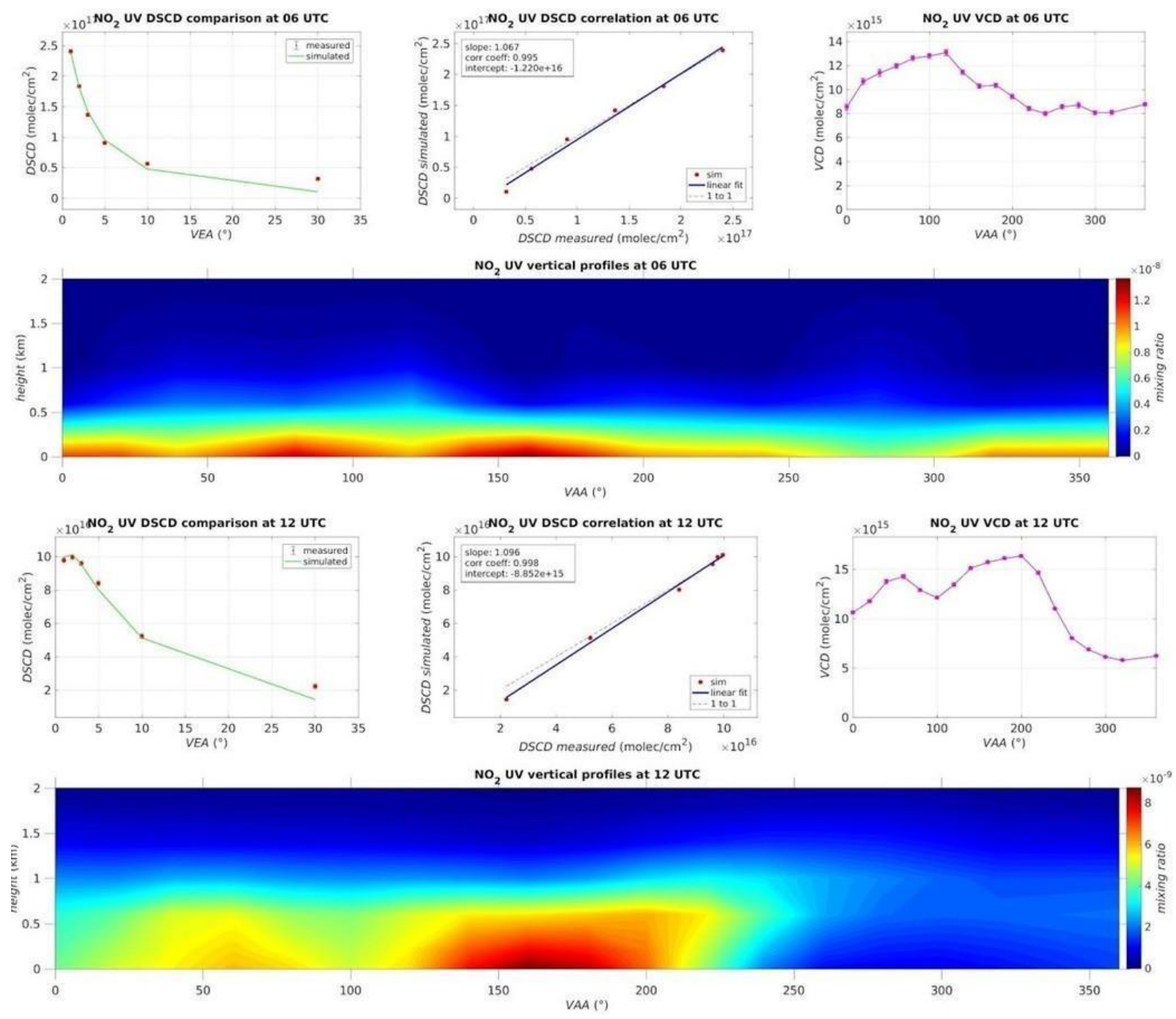

Figure 8.8. $\mathrm{NO}_{2}$ vertical distribution retrieved in the UV region at 6 UTC and at 12 UTC on May 11, 2019. These contour plots are smoothed from adjacent VAA data points separated by $20^{\circ}$ in order to estimate the azimuthal distribution of $\mathrm{NO}_{2}$ over Madrid.

We have also analyzed HONO DSCDs measured by the MAXDOAS-2D using the same configuration as in Garcia-Nieto et al., 2018. Figure 8.9 shows a two-dimensional representation of HONO on May 11, 2019 at 6 UTC. We retrieve surface layer peak values 
of 0.7 ppbv pointing at $50^{\circ}$ of VAA in the early morning, in agreement with previous studies for HONO in urban environments (see Hendrick et al., 2014; Ryan et al., 2018). The VCDs at 6 UTC range from $6 \times 10^{14}$ to $1.2 \times 10^{15}$ molecule $\mathrm{cm}^{-2}$. The combination of spatially distributed measurements of $\mathrm{NO}_{2}$ and $\mathrm{HONO}$ can be used together with chemical transport models to further understand pollution dynamics in Madrid.
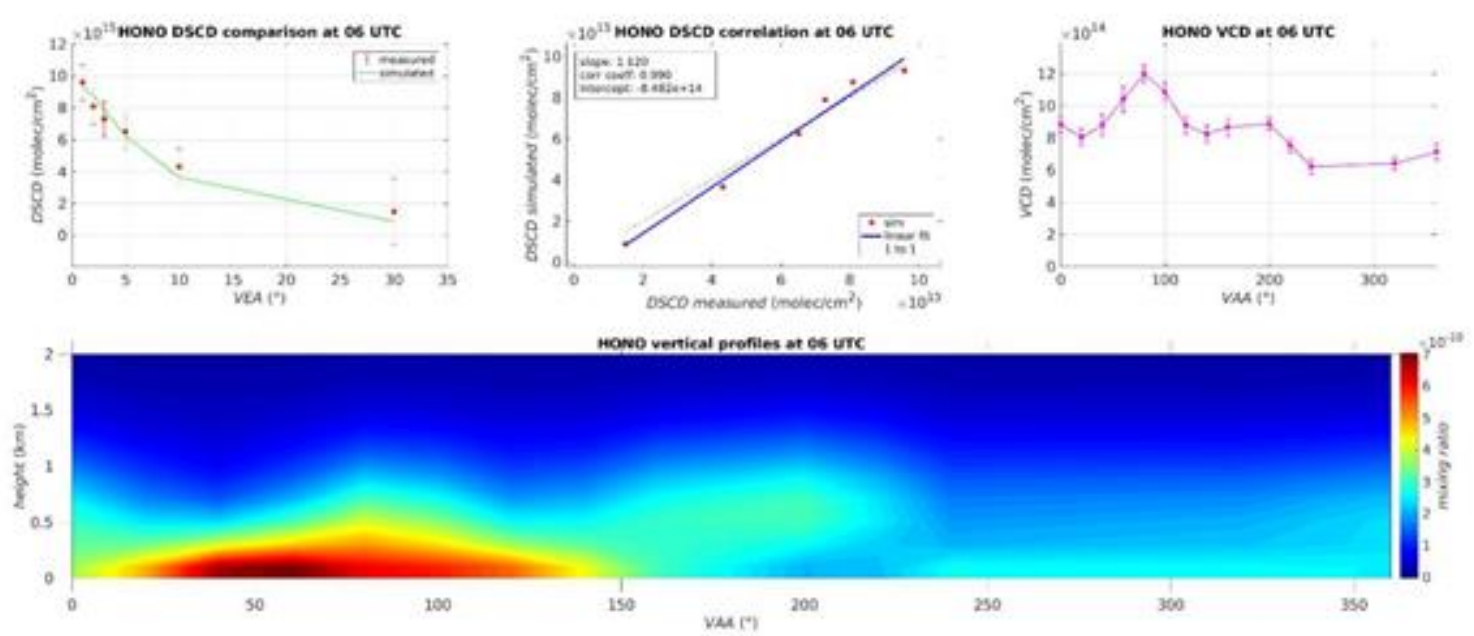

Figure 8.9. HONO vertical distribution retrieved at 6 UTC.

\subsubsection{Horizontal distribution of $\mathrm{NO}_{2}$}

Based on Equation (8.2), we derive the horizontal distribution of $\mathrm{NO}_{2}$ in the surface layer (0-200 m height). Figure 8.10 shows an example of surface layer $\mathrm{NO}_{2}$ mixing ratios over two radial distances from the MAXDOAS-2D instrument (using the UV and VIS $\mathrm{NO}_{2}$, respectively, as explained in Subsection 8.3.3), located at the center of the plot. The highest mixing ratios occur during the first sunlit hours (7-8 UTC), coincident with the peak in $\mathrm{NO}_{x}$ emissions in Madrid (Quaassdorff et al., 2016). This early morning peak is followed by a gradual decrease in surface layer $\mathrm{NO}_{2}$ mixing ratios during the day. Note that $\mathrm{NO}_{2}$ is predominantly located in the southern part of the semisphere (VAA from $90^{\circ}$ to $270^{\circ}$ ). 

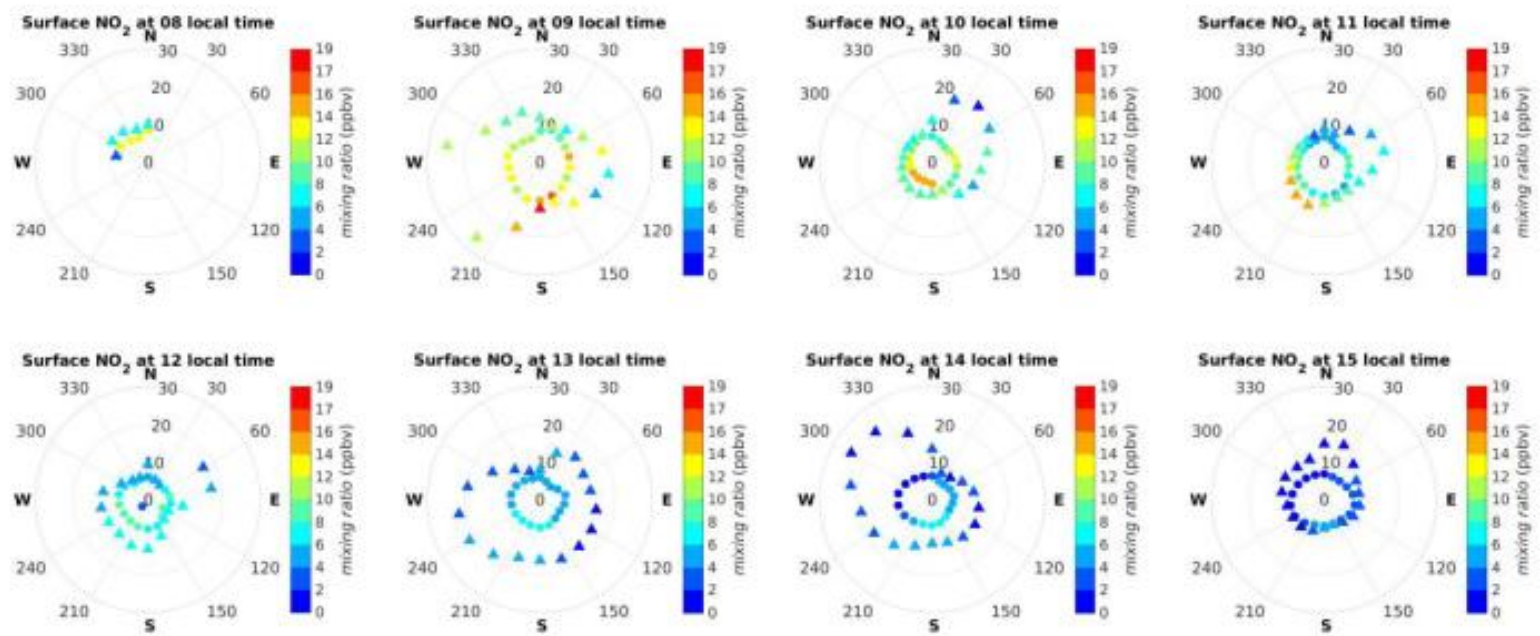

Figure 8.10. Polar plots of $\mathrm{NO}_{2}$ within the surface layer (0-200 $\mathrm{m}$ height) for May 11, 2019. Please note that these polar plots extend over a direction perpendicular to those shown in Figure 8.8. Here, circles are used for the UV (shorter horizontal light path) and triangles for the VIS (larger horizontal light path).

In order to make it easier to visualize the changes and transport of $\mathrm{NO}_{2}$ masses, Figure 8.11 includes a contour plot interpolating the polar plot data of the previous figure. 

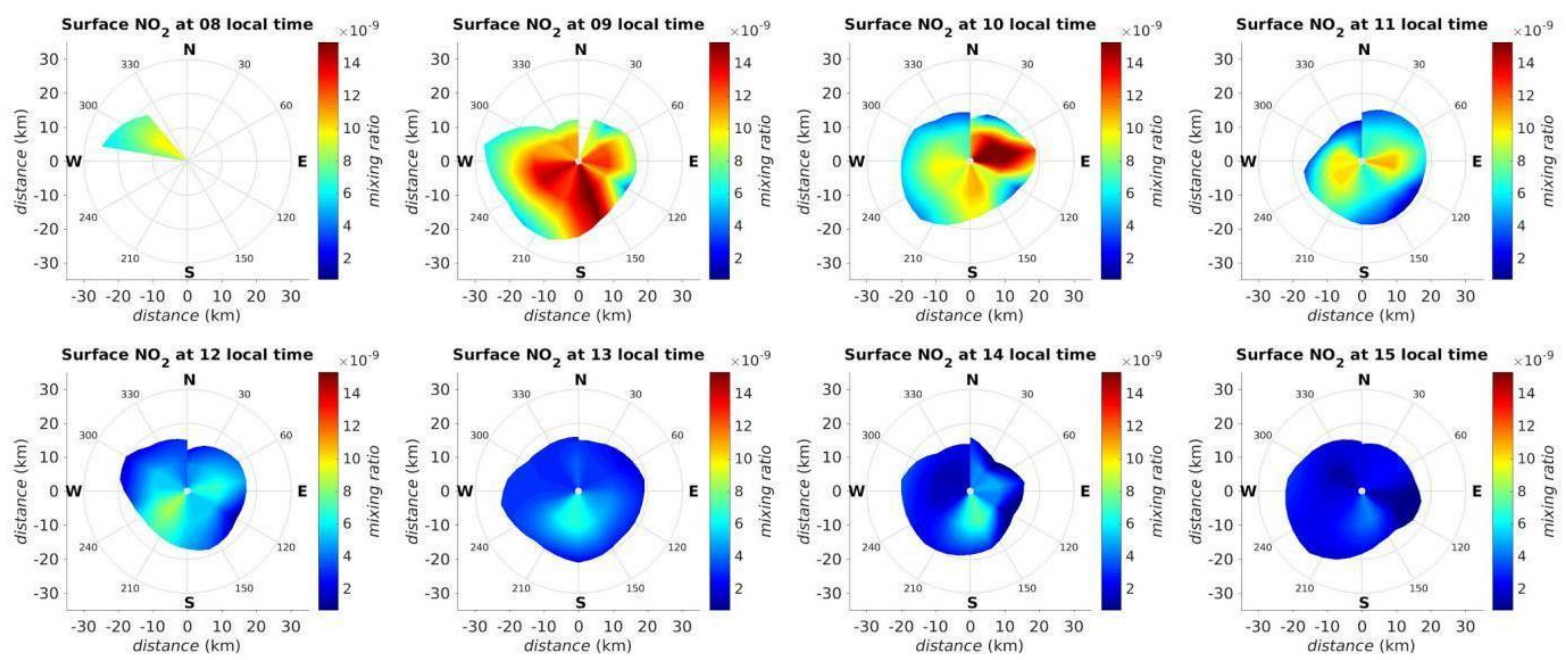

Figure 8.11. Polar plots of $\mathrm{NO}_{2}$ within the surface layer (0-200 $\mathrm{m}$ height) for May 11, 2019. Please note that these contours extend over a direction perpendicular to those shown in Figure 8.8. These contour plots are smoothed from adjacent VAA data points separated by $20^{\circ}$ and from the two different horizontal light paths in the radial direction.

\subsubsection{Correlation with the in-situ air quality monitoring stations of Madrid}

The City Council of Madrid (CCM) monitors the air quality throughout the city: the monitoring network consists of 27 stations that form the Air Quality Network (see Figure 8.12). 


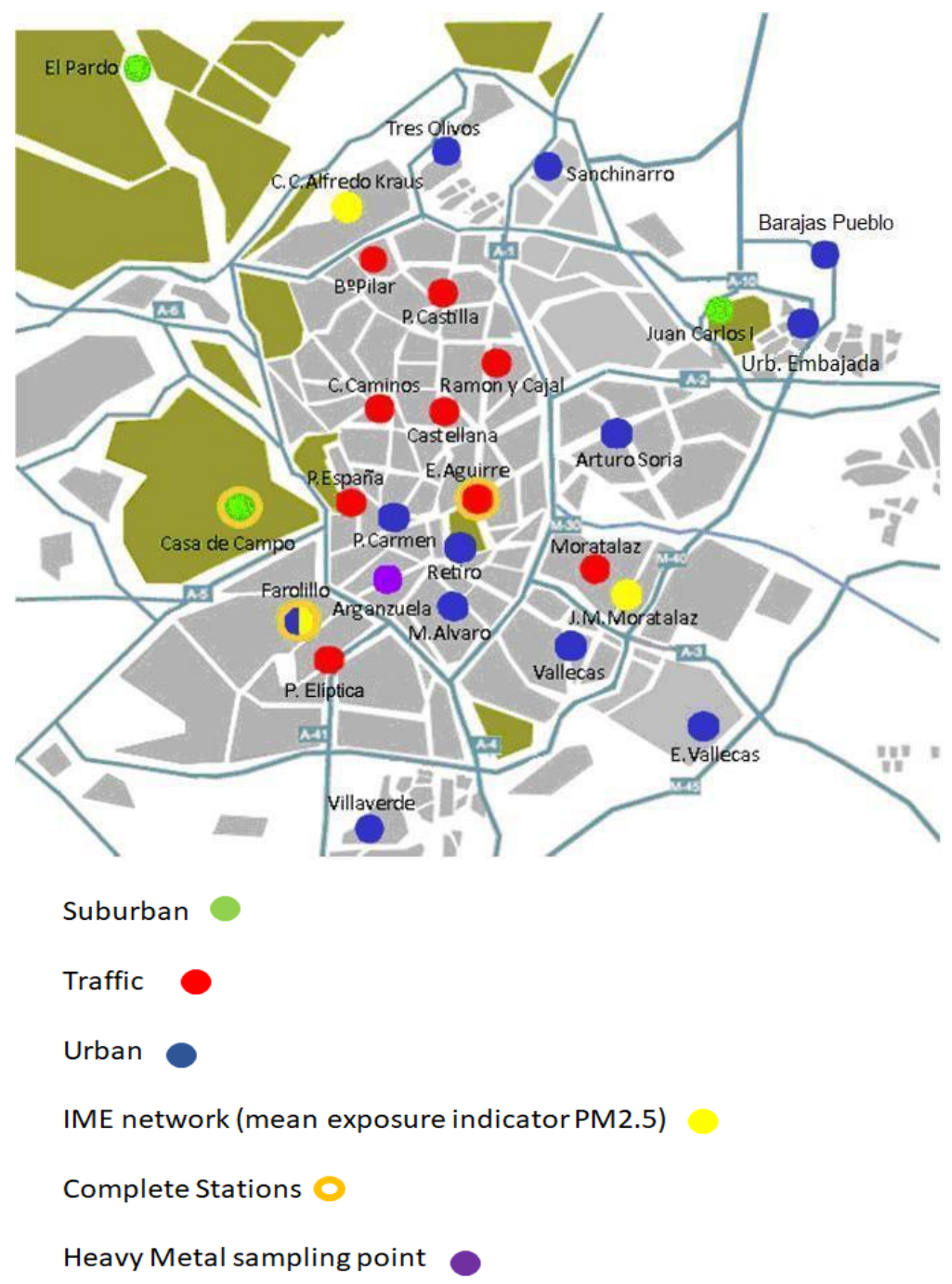

Figure 8.12. Monitoring stations in Madrid, the picture was taken from (c) Annual Plan 2019 Madrid.

We suggest that MAXDOAS-2D mesoscale observations may complement the information provided by the local air quality monitoring network based on reference analytical techniques (according to Directive 2008/50/EC). While air quality monitors of the reference network provide information about ambient concentrations in their specific locations (currently 24 air quality monitoring stations measure $\mathrm{NO}_{2}$ within the city, see $\mathrm{AM}$, 2019), MAXDOAS-2D observations produce near ground-level concentrations averaged over the optical path in a given direction. That prevents us from quantitatively comparing both types of observations. Nonetheless, we analyzed their correspondence using the $\mathrm{NO}_{2}$ 
concentrations measured by the in-situ instruments throughout the entire city, and the $\mathrm{NO}_{2}$ mixing ratios within the surface layer derived from our MAXDOAS-2D instrument over the 2-month period (May-June, 2019). For this comparison, we considered the air quality monitoring stations within a distance from the MAXDOAS-2D equal or lower than $10 \mathrm{~km}$. Since this is the typical horizontal light path for the UV region, we decided to include only the $\mathrm{NO}_{2}$ values retrieved in the UV region for the comparison. Strong gradients between the values measured by the in-situ instruments are typical. Therefore, and considering that we are mainly interested in their temporal correlation with respect to our MAXDOAS-2D measurements, we compare both the in-situ $\mathrm{NO}_{2}$ and surface layer MAXDOAS-2D hourlyaveraged data. Note that for the MAXDOAS-2D this approximately corresponds to averaging the surface layer values for each azimuthal lap, given that each azimuthal lap takes approximately 1 hour to complete.

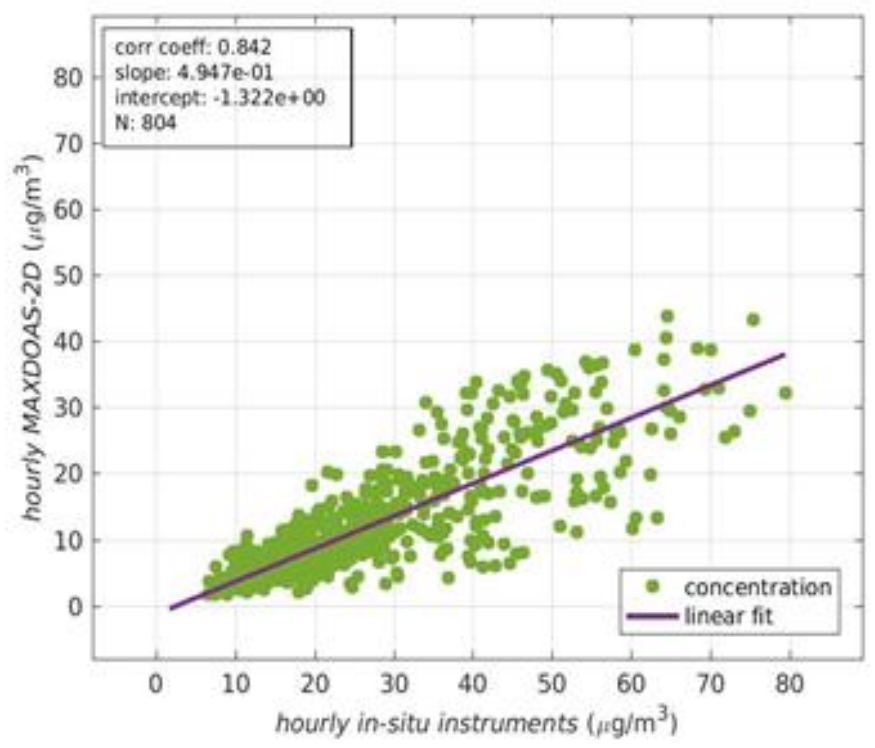

Figure 8.13. Correlation between in-situ observations from Madrid's air quality monitoring network and those derived from the MAXDOAS-2D instrument for the surface layer (0-200 $\mathrm{m}$ height).

Despite the different spatial representativeness, Figure 11 shows a reasonably good correlation coefficient of 0.842 between both datasets for the two-month campaign. The 
slope is lower than 1 , this can be explained by the typical $\mathrm{NO}_{2}$ vertical profiles in urban environments. Simulations performed over Madrid with a high-resolution Eulerian air quality model (Borge et al., 2018) yielded an exponentially decreasing with height $\mathrm{NO}_{2}$ profile. Therefore, the MAXDOAS-2D mixing ratios, which represent an average across the surface layer (0-200 m height), are not expected to quantitatively match the values of insitu instruments, located close to the surface (between 0-10 m height). Similar conclusions -and slopes comparable to the one retrieved above- regarding the correlation between insitu and MAXDOAS instruments can be found in previous works (Schreier et al., 2019; Kramer et al., 2008; Chan et al., 2020). In addition, there is a good temporal correlation between in-situ and MAXDOAS-2D measurements over an extended period of time. 


\section{CONCLUSIONS AND FUTURE}

\section{WORK}

This thesis reports on remote-sensing measurements of trace gases in the atmosphere of Madrid and their assessment. The ground-based Multi AXis Differential Optical Absorption Spectroscopy technique has been used. The objectives achieved and the main results described across the different chapters are summarized below.

A comprehensive analysis of $\mathrm{NO}_{2}, \mathrm{HONO}$, glyoxal and formaldehyde vertical concentration profiles in the urban atmosphere of Madrid (Spain) has been performed. Our MAXDOAS-1D instrument (located within the city centre) was operating during the entire year of 2016 allowing the retrieval of diurnal and seasonal cycles of these trace gases. Using DOAS spectral fitting methods and a radiative transfer model, information on vertical columns and their altitude-distributed concentrations were retrieved.

Regarding nitrous acid, the year-round simulation yielded a good agreement with the DSCDs measured for both $\mathrm{O}_{4}, \mathrm{NO}_{2}$ and $\mathrm{HONO}$, with correlation coefficients close to 0.95. $\mathrm{NO}_{2}$ seasonality shows higher mixing ratios in winter and autumn (from $12 \mathrm{ppbv}$ up to $78 \mathrm{ppbv}$ ), while during spring and summer the $\mathrm{NO}_{2}$ mixing ratios range between 8 and 38 ppbv. The seasonality of HONO has also been studied showing higher concentration values between 2 and 4 ppbv of within the first layer (up to $100 \mathrm{~m}$ height) in autumn and winter with respect to spring and summer. The latter seasons showed relatively high peak values in the early morning and afternoon, however the photolysis impact is greater in these months, causing a more significant HONO loss around noon. A similar behaviour was observed in the seasonal vertical profiles at a given time (8 UTC), providing a clear gradient 
among seasons and similar peak values. This seasonal variation also appears in the monthly evolution both of the first layer relative concentration and VCD. Finally, an assessment of the contribution of $\mathrm{HONO}$ photolysis to the $\mathrm{OH}$ formation through photolysis was also performed. Maximum averaged values close to 1 pptv $\mathrm{s}^{-1}$ were found in autumn around noon. Moreover, values higher than 0.5 pptv s $^{-1}$ were observed for every season around noon, which are within the range of previous modelling reports over heavily polluted regions.

The seasonal evolution of formaldehyde surface mixing ratios showed two distinct peaks for each season (in the early morning and late afternoon) and minimum values around noon, with higher levels of $\mathrm{HCHO}$ during autumn and winter, particularly during the afternoon. Daytime mixing ratios ranged between $6 \mathrm{ppbv}$ (in spring at $13 \mathrm{UTC}$ ) and $30 \mathrm{ppbv}$ (in autumn at 07 UTC). The seasonal evolution of HCHO VCDs had similar trends to the surface mixing ratios varying from $1 \times 10^{16}$ molecules $\mathrm{cm}^{-2}$ (in spring at $13 \mathrm{UTC}$ ) to $5 \times 10^{16}$ molecules $\mathrm{cm}^{-2}$ (in autumn at 14 UTC). The glyoxal DOAS analysis is challenging due to strong water vapour absorption interference. This factor, along with instrumental saturation problems and the limited values that remained after the quality checks restricted the seasonal analysis to certain hours in the early morning. For this reason, only monthly averages at 09 UTC have been included, showing a winter peak value of 2 ppbv, whereas the minimum mixing ratios were found in August, 0.7 ppbv.

We also evaluated ratios between $\mathrm{CHOCHO}$ and $\mathrm{HCHO}$ surface mixing ratios in order to estimate the nature of their VOCs precursors. Values within the usual ranges reported in the literature for urban sites were found, with a higher relative contribution from biogenic sources of VOCs observed in this study during spring and summer. Lower levels in autumn and winter. Two additional analyses were carried out in order to assess the meaning of $R_{G F}$, and we found that the $\mathrm{R}_{\mathrm{GF}}$ trend was anticorrelated with the $\mathrm{NO}_{2}$ surface mixing ratios -which are directly involved in anthropogenic emissions- and correlated with the ratio between biogenic and anthropogenic VOCs emission inventories for the city. 
Nevertheless, there are discrepancies in the interpretation of this ratio which points to the need for more simultaneous observations of VOCs precursors, $\mathrm{HCHO}$ and $\mathrm{CHOCHO}$ under different biogenic and anthropogenic emission environments.

The work compiled in this thesis demonstrates that the MAXDOAS-1D represents a useful instrument for atmospheric measurements since it is able to analyse several trace gases at the same time in different spectral regions. In particular, this MAXDOAS-1D instrument will continue the observations to establish a long term series of relevant trace gases and aerosols in Madrid.

Within the course of this thesis, we have fully developed for the first time a MAXDOAS-2D instrument to obtain two-dimensional maps of air pollution in the city of Madrid. A comprehensive analysis of $\mathrm{O}_{4}, \mathrm{NO}_{2}$ and $\mathrm{HONO}$ vertical concentration profiles in the urban atmosphere of Madrid (Spain) has been performed over two months (from May 6 to July 5, 2019). We analyzed the absorptions and derived the corresponding DSCDs for these trace gases. Then, the corresponding profiles were retrieved using a RTM. In this step, we assessed the impact of different atmospheric profiles (pressure and temperature) in the retrieval results. The overall comparison of measured and simulated DSCDs showed that they were in very good agreement in all cases, supporting the reliability of the observations.

This new MAXDOAS-2D was built from scratch in our laboratory and represents the first application of this remote sensing technology for air pollution research in Spain. Both the software and the hardware were adapted to our location. We also installed a tower in order to avoid the high buildings which were in the instrumental field of view. We have also inferred information on the horizontal gradient of $\mathrm{NO}_{2}$ within the surface layer making use of the strong dependence between wavelengths and light paths across the $\mathrm{NO}_{2}$ absorption spectrum. The resulting "onion-peeling" figures indicate peak values of $\mathrm{NO}_{2}$ in the early morning and in the southern section of the city (around $180^{\circ}$ of VAA). We suggest that the 
new mesoscale information provided by the MAXDOAS-2D instrument helps in the study of pollution transport dynamics in Madrid.

As future work, we will analyse these trace gases measured by the MAXDOAS-1D for a long term period (4-5 years) and establish relationships with COVs and traffic emissions. The future work with the MAXDOAS-2D involves the analysis of other trace gases such as glyoxal and formaldehyde. Together, we will continue the operation of the instrument to build a time series of the vertical and horizontal distribution of these pollutants over the city of Madrid. 


\section{REFERENCES}

Acker, K., Febo, A., Trick, S., Perrino, C., Bruno, P., Wiesen, P., Möller, D., Wieprecht, W., Auel, R., Giusto, M., Geyer, A., Platt, U., Allegrini, I. (2006). Nitrous acid in the urban area of Rome. Atmos. Environ. 40 (17), 3123-3133.

Alicke, B., Geyer, A., Hofzumahaus, A., Holland, F., Konrad, S., Pätz, H.W., Schäfer, J., Stutz, J., Volz-Thomas, A., Platt, U. (2003). OH formation by HONO photolysis during the BERLIOZ experiment, J. Geophys. Res. Atmos. 108 pp. PHO 3-1; PHO 3-17.

Aliwell, S. R., Van Roozendael, M., Johnston, P. V., Richter, A., Wagner, T., Arlander, D. W., Burrows, J. P., Fish, D. J., Jones, R. L., Tørnkvist, K. K., Lambert, J. C., Pfeilsticker, K., \& Pundt, I. (2002). Analysis for BrO in zenith-sky spectra: An intercomparison exercise for analysis improvement. Journal of Geophysical Research Atmospheres. https://doi.org/10.1029/2001JD000329.

Arpaq, K. H., Johnston, P. V., Miller, H. L., Sanders, R. W., and Solomon, S.(1994). Observations of the stratospheric BrO column overColorado, 40॰N, J. of Geophys. Res., 99, D4, 8175-8181.

Atkinson, R., Baulch, D.L., Cos, R.A., Crowley, J.N., Hampson, R.F., Haynes, R.G., Jenkin, M.E., Rossi, M.J., Troe, J. (2004). Evaluated kinetic and photochemical data for atmospheric chemistry: volume IN - gas phase reactions of $\mathrm{O}_{\mathrm{x}}, \mathrm{HO}_{\mathrm{x}}, \mathrm{NO}_{\mathrm{x}}$ and $\mathrm{SO}_{\mathrm{x}}$ species. Atmos. Chem. Phys. 4 (6), 1461-1738. 
Ayuntamiento de Madrid (AM). (2019). Madrid 2016 Annual Air Quality Assessment Report (Calidad del aire Madrid 2019). General Directorate of Sustainability and Environmental Control, Madrid City Council Available online -only Spanish version-at http://www.mambiente.munimadrid.es/opencms/export/sites/default/calaire/Anexos/M emorias/Memoria_2019.pdf.

Bakeas, E.B., Argyris, D.I., Siskos, P.A. (2003). Carbonyl compounds in the urban environment of Athens, Greece. Chemosphere 52 (5), 805-813.

Beer, A. (1854). Grundriss des photometrischen Calcüles, Vieweg.

Benavent, N., Garcia-Nieto, D., Wang, S., \& Saiz-Lopez, A. (2019). MAX-DOAS measurements and vertical profiles of glyoxal and formaldehyde in Madrid, Spain. Atmospheric Environment, 199, 357-367. https://doi.org/10.1016/j.atmosenv.2018.11.047

Borge, R., Lumbreras, J., Rodríguez, E. (2008). Development of a high-resolution emission inventory for Spain using the SMOKE modelling system: a case study for the years 2000 and 2010. Environ. Model. Software 23 (8), 1026-1044.

Borge, R., Narros, A., Artiñano, B., Yagüe, C., Gómez-Moreno, F.J., de la Paz, D., RománCascón, C., Díaz, E., Maqueda, G., Sastre, M., Quaassdorff, C., Dimitrolopoulou, C., Vardoulakis, S., (2016). Assessment of microscale spatio-temporal variation of air pollution at an urban hotspot in Madrid (Spain) through an extensive field campaign. Atmos. Environ. $140,432-445$.

Borge, R., Santiago, J.L., de la Paz, D., Martín, F., Domingo, J., Valdés, C., Sánchez, B., Rivas, E., Rozas, M.T., Lázaro, S., Pérez, J., Fernández, Á., (2018). Application of a short term air 
quality action plan in Madrid (Spain) under a high-pollution episode - Part II: assessment from multi-scale modelling. Sci. Total Environ. 635, 1574-1584.

Brooks, S. B., Saiz-Lopez, A., Skov, H., Lindberg, S. E., Plane, J. M. C., and Goodsite, M. E. (2006). The mass balance of mercury in the springtime arctic environment, Geophys. Res. Lett., 33, L13812, doi:10.1029/2005GL025525.

Carnerero, C., Pérez, N., Petäjä, T., Laurila, T.M., Ahonen, L.R., Kontkanen, J., Ahn, K.- H., Alastuey, A., Querol, X. (2019). Relating high ozone, ultrafine particles, and new particle formation episodes using cluster analysis. Atmos. Environ. X, 100051.

Chan, K. L., Wiegner, M., van Geffen, J., De Smedt, I., Alberti, C., Cheng, Z., Ye, S., and Wenig, M. (2020). MAX-DOAS measurements of tropospheric $\mathrm{NO}_{2}$ and $\mathrm{HCHO}$ in Munich and the comparison to OMI and TROPOMI satellite observations, Atmos. Meas. Tech., 13, 44994520, https://doi.org/10.5194/amt-13-4499-2020.

Chance, K., Kurucz, R.L. (2010). An improved high-resolution solar reference spectrum for earth's atmosphere measurements in the ultraviolet, visible, and near infrared. J. Quant. Spectrosc. Radiat. Transf.; Special Issue Dedicated to Laurence S. Rothman on the Occasion of his 70th Birthday 111 (9), 1289-1295.

Chance, K. V., \& Spurr, R. J. D. (1997). Ring effect studies: Rayleigh scattering, including molecular parameters for rotational Raman scattering, and the Fraunhofer spectrum. Applied Optics. https://doi.org/10.1364/ao.36.005224.

Clémer, K., Van Roozendael, M., Fayt, C., Hendrick, F., Hermans, C., Pinardi, G., Spurr, R., Wang, P., De Mazière, M., (2010). Multiple wavelength retrieval of tropospheric aerosol optical properties from MAX-DOAS measurements in Beijing. Atmos. Meas. Tech. 3 (4), 863.

Coburn, S., Ortega, I., Thalman, R., Blomquist, B., Fairall, C.W., Volkamer, R. (2014). Measurements of diurnal variations and eddy covariance (EC) fluxes of glyoxal in the 
tropical marine boundary layer: description of the Fast LED-CE-DOAS instrument. Atmospheric Measurement Techniques 7, 3579-3595.

Cuevas, C. A., Maffezzoli, N., Corella, J. P., Spolaor, A., Vallelonga, P., Kjær, H. A., Simonsen, M., Winstrup, M., Vinther, B., Horvat, C., Fernandez, R. P., Kinnison, D., Lamarque, J. F., Barbante, C., \& Saiz-Lopez, A. (2018). Rapid increase in atmospheric iodine levels in the North Atlantic since the mid-20th century. Nature Communications. https://doi.org/10.1038/s41467-018-03756-1.

de la Paz, D., Borge, R., \& Martilli, A. (2016). Assessment of a high resolution annual WRF$\mathrm{BEP} / \mathrm{CMAQ}$ simulation for the urban area of Madrid (Spain). Atmospheric Environment, 144, 282-296. https://doi.org/10.1016/j.atmosenv.2016.08.082.

Deutschmann, T., Beirle, S., Friess, U., Grzegorski, M., Kern, C., Kritten, L., Platt, U., PradosRoman, C., Puḳīte, J., Wagner, T., Werner, B., \& Pfeilsticker, K. (2011). The Monte Carlo atmospheric radiative transfer model McArtim: Introduction and validation of Jacobians and 3D features. Journal of Quantitative Spectroscopy \& Radiative Transfer, 112, 11191137.

DiGangi, J.P., Henry, S.B., Kammrath, A., Boyle, E.S., Kaser, L., Schnitzhofer, R., Graus, M., Turnipseed, A., Park, J.-H., Weber, R.J., Hornbrook, R.S., Cantrell, C.A., Maudlin III, R.L., Kim, S., Nakashima, Y., Wolfe, G.M., Kajii, Y., Apel, E.C., Goldstein, A.H., Guenther, A., Karl, T., Hansel, A., Keutsch, F.N. (2012). Observations of glyoxal and formaldehyde as metrics for the anthropogenic impact on rural photochemistry. Atmos. Chem. Phys. 12, 9529-9543.

Dimitropoulou, E., Hendrick, F., Pinardi, G., Friedrich, M. M., Merlaud, A., Tack, F., De Longueville, H., Fayt, C., Hermans, C., Laffineur, Q., Fierens, F., and Van Roozendael, M. (2020). Validation of TROPOMI tropospheric $\mathrm{NO}_{2}$ columns using dual-scan multi-axis differential optical absorption spectroscopy (MAX-DOAS) measurements in Uccle, Brussels, Atmos. Meas. Tech., 13, 5165-5191, https://doi.org/10.5194/amt-13-5165-2020. 
Dufour, G., Wittrock, F., Camredon, M., Beekmann, M., Richter, A., Aumont, B., Burrows, J.P., (2009). SCIAMACHY formaldehyde observations: constraint for isoprene emission estimates over Europe?

European Environment Agency (EEA), (2019). Air quality in Europe - 2019 report. Catalogue number: TH-AL-19-012-EN-N. ISBN: 978-92-9480-088-6. Available online at: http://www.eea.europa.eu/publications/air-quality-in-europe-2019.

Fleischmann, O.C., Hartmann, M., Burrows, J.P., Orphal, J. (2004). New ultraviolet absorption cross-sections of $\mathrm{BrO}$ at atmospheric temperatures measured by timewindowing Fourier transform spectroscopy. J. Photochem. Photobiol. Chem. 168 (1-2), 117132.

Garzón, J.P., Huertas, J.I., Magaña, M., Huertas, M.E., Cárdenas, B., Watanabe, T., Maeda, T., Wakamatsu, S., Blanco, S. (2015). Volatile organic compounds in the atmosphere of Mexico city. Atmos. Environ. 119, 415-429.

Gomez, L., Navarro-Comas, M., Puentedura, O., Gonzalez, Y., Cuevas, E., and Gil-Ojeda, M. (2014). Long-path averaged mixing ratios of $\mathrm{O}_{3}$ and $\mathrm{NO}_{2}$ in the free troposphere from mountain MAX-DOAS, Atmos. Meas. Tech., 7, 3373-3386, https://doi.org/10.5194/amt-73373-2014.

González Abad, G., Liu, X., Chance, K., Wang, H., Kurosu, T.P., Suleiman, R., (2015). Updated Smithsonian astrophysical observatory ozone monitoring instrument (SAO OMI) formaldehyde retrieval. Atmospheric Measurement Techniques 8, 19-32.

Gouw, J.A., Gilman, J.B., Kim, S.-W., Alvarez, S.L., Dusanter, S., Graus, M., Griffith, S.M., Isaacman-VanWertz, G., Kuster, W.C., Lefer, B.L., Lerner, B.M., McDonald, B.C., Rappenglück, B., Roberts, J.M., Stevens, P.S., Stutz, J., Thalman, R., Veres, P.R., Volkamer, 
R., Warneke, C., Washenfelder, R.A., Young, C.J., (2018). Chemistry of volatile organic compounds in the Los Angeles basin: formation of oxygenated compounds and determination of emission ratios. J. Geophys. Res: Atmosphere 123 (4), 2298-2319.

Grainger, J. F., and Ring, J., (1962). The Physics and Astronomy of the Moon, edit. by Z. Kopal, 383, Academic Press.

Garcia-Nieto, D., Benavent, N., \& Saiz-Lopez, A. (2018). Measurements of atmospheric HONO vertical distribution and temporal evolution in Madrid (Spain) using the MAX-DOAS technique. Science of the Total Environment, 643. https://doi.org/10.1016/j.scitotenv.2018.06.180.

Gratsea, M., Vrekoussis, M., Richter, A., Wittrock, F., Schönhardt, A., Burrows, J., Kazadis, S., Mihalopoulos, N., Gerasopoulos, E. (2016). Slant column MAX-DOAS measurements of nitrogen dioxide, formaldehyde, glyoxal and oxygen dimer in the urban environment of Athens. Atmos. Environ. 135, 118-131.

Grosjean, D., Miguel, A.H., Tavares, T.M. (1990). Urban air pollution in Brazil: acetaldehyde and other carbonyls. Atmospheric Environment. Part B. Urban Atmosphere 24 (1), 101-106.

Grosjean, D. (1991). Ambient levels of formaldehyde, acetaldehyde and formic acid in southern California: results of a one-year baseline study. Environ. Sci. Technol. 25 (4), 710715.

Grosjean, E., Grosjean, D., Fraser, M.P., Cass, G.R. (1996). Air quality model evaluation data for organics. 2.C1 - C14 carbonyls in Los Angeles air. Environ. Sci. Technol. 30 (9), 26872703.

Guenther, A.B., Jiang, X., Heald, C.L., Sakulyanontvittaya, T., Duhl, T., Emmons, L.K., Wang, X., (2012). The Model of Emissions of Gases and Aerosols from Nature version 2.1 
(MEGAN2.1): an extended and updated framework for modeling biogenic emissions. Geosci. Model Dev. (GMD) 5, 1471-1492.

Heard, D.E., Pilling, M.J. (2003). Measurement of $\mathrm{OH}$ and $\mathrm{HO} 2$ in the troposphere. Chem. Rev. 103 (12), 5163-5198.

Heckel, A., Richter, A., Tarsu, T., Wittrock, F., Hak, C., Pundt, I., Junkermann, W., Burrows, J.P., (2005). MAX-DOAS measurements of formaldehyde in the Po- Valley. Atmos. Chem. Phys. 5, 909-918.

Henyey L. G., and Greenstein J L. (1941). Diffuse radiation in the galaxy Astrophys. J., 93, 70-83.

Heland, J., Kleffmann, J., Kurtenbach, R., Wiesen, P. (2001). A new instrument to measure gaseous nitrous acid (HONO) in the atmosphere. Environ. Sci. Technol. 35 (15), 3207-3212.

Hendrick, F., Müller, J.-., Clémer, K., Wang, P., De Mazière, M., Fayt, C., Gielen, C., Hermans, C., Ma, J., Pinardi, G., Stavrakou, T., Vlemmix, T., Van Roozendael, M. (2014). Four years of ground-based MAX-DOAS observations of HONO and NO2 in the Beijing area. Atmos. Chem. Phys. 14 (2), 765.

Ho, K.F., Lee, S.C., Louie, P.K.K., Zou, S.C. (2002). Seasonal variation of carbonyl compound concentrations in urban areas of Hong Kong. Atmos. Environ. 36 (8), 1259-1265.

Ho, S.S.H., Yu, J.Z. (2002). Feasibility of collection and analysis of airborne carbonyls by onsorbent derivatization and thermal desorption. Anal. Chem. 74 (6), 1232-1240.

Hönninger, G., von Friedeburg, C., Platt, U. (2004). Multi axis differential optical absorption spectroscopy (MAXDOAS). Atmos. Chem. Phys. 4 (1), 231-254. 
Honsberg, C.B., and Bowden, S.G. (2019). page on www.pveducation.org.

Irie, H., Takashima, H., Kanaya, Y., Boersma, K.F., Gast, L., Wittrock, F., Brunner, D., Zhou, Y., Van Roozendael, M. (2011). Eight-component retrievals from ground-based MAX-DOAS observations. Atmos. Measurement Technique 4, 1027-1044.

Izquierdo, R., García Dos Santos, S., Borge, R., Paz, D. de la, Sarigiannis, D., Gotti, A., \& Boldo, E.(2020). Health impact assessment by the implementation of Madrid City airquality plan in 2020. Environmental Research, 183, 109021. https://doi.org/10.1016/j.envres.2019.109021.

Kaiser, J., Wolfe, G.M., Min, K.E., Brown, S.S., Miller, C.C., Jacob, D.J., DeGouw, J.A., Graus, M., Hanisco, T.F., Holloway, J., Peischl, J., Pollack, I.B., Ryerson, T.B., Warneke, C., Washenfelder, R.A., Keutsch, F.N. (2015). Reassessing the ratio of glyoxal to formaldehyde as an indicator of hydrocarbon precursor speciation. Atmos. Chem. Phys. 15 (13), 75717583.

Kasten, F., Young, A.T., (1989). Revised optical air mass tables and approximation formula. Appl. Opt. 28 (22), 4735-4738.

Khokhar, M.F., Naveed, S.L., Butt, J.K., Abbas, Z. (2016). Comparative analysis of atmospheric glyoxal column densities retrieved from MAX-DOAS observations in Pakistan and during MAD-CAT field campaign in Mainz, Germany. Atmosphere 7, 68.

Kielhorn, J., Pohlenz-Michel, C., Schmidt, S., Mangelsdorf, I. (2004). "Glyoxal”, Concise International Chemical Assessment, Doc. 57, Int. Programme on Chem.-Safety. World Health Organization, Geneva. 
Kramer, L. J., Leigh, R. J., Remedios, J. J., and Monks, P. S. (2008). Comparison of OMI and ground-based in situ and MAX-DOAS measurements of tropospheric nitrogen dioxide in an urban area, J. Geophys. Res., 113, D16S39, doi:10.1029/2007JD009168.

Kurtenbach, R., Becker, K.H., Gomes, J.A.G., Kleffmann, J., Lörzer, J.C., Spittler, M., Wiesen, P., Ackerman, R., Geyer, A., Platt, U. (2001). Investigations of emissions and heterogeneous formation of HONO in a road traffic tunnel. Atmos. Environ. 35 (20), 3385-3394.

Lee, H., Ryu, J., Irie, H., Jang, S.-H., Park, J., Choi, W., Hong, H. (2015). Investigations of the diurnal variation of vertical $\mathrm{HCHO}$ profiles based on MAXDOAS measurements in Beijing: comparisons with OMI vertical column data. Atmosphere 6, 1816-1832.

Lee, J.D., Whalley, L.K., Heard, D.E., Stone, D., Dummore, R.E., Hamilton, J.F., Young, D.E., Allan, J.D., Laufs, S., Kleffmann, J. (2016). Detailed budget analysis of HONO in central London reveals a missing daytime source. Atmos. Chem. Phys. 16, 2747-2764.

Leighton, P. (1961). Photochemistry of air pollution. New York: Academic Press.

Liu, W., Zhang, J., Kwon, J., Weisel, C., Turpin, B., Zhang, L., Korn, L., Morandi, M., Stock, T., Colome, S. (2006). Concentrations and source characteristics of airborne carbonyl compounds measured outside urban residences. J. Air Waste Manag. Assoc. 56 (8), 11961204.

Maddox, E. M., and Mullendore, G.L. (2018). Determination of Best Tropopause Definition for Convective Transport Studies. J. Atmos. Sci., 75, 3433-3446, https://doi.org/10.1175/JAS-D-18-0032.1.

Mahajan, A.S., Prados-Roman, C., Hay, T.D., Lampel, J., Pöhler, D., GroBmann, K., Tschritter, J., FrieB, U., Platt, U., Johnston, P., Kreher, K., Wittrock, F., Burrows, J.P., Plane, J.M.C., Saiz- 
Lopez, A. (2014). Glyoxal observations in the global marine boundary layer. J. Geophys. Res: Atmosphere 119 (10), 6160-6169.

Meller, R., Moortgat, G.K. (2000). Temperature dependence of the absorption cross sections of formaldehyde between 223 and $323 \mathrm{~K}$ in the wavelength range $225-375 \mathrm{~nm}$. J. Geophys. Res.: Atmosphere 105, 7089-7101.

Mie, G. (1908). On the optics of turbid media, especially colloidal metal solutions. Ann. Phys. Berlin.

Miller, C.C., Gonzalez Abad, G., Wang, H., Liu, X., Kurosu, T., Jacob, D.J., Chance, K. (2014). Glyoxal retrieval from the ozone monitoring instrument. Atmospheric Measurement Techniques 7, 3891-3907.

Miller, H. L., Weaver, A., Sanders, R. W., Arpag, K., and Solomon, S. (1997) Measurements of arctic sunrise surface ozone depletion events at Kangerlussuaq, Greenland (67॰N, 51॰W), Tellus, 49B,496-509.

Monks, P.S., Granier, C., Fuzzi, S., Stohl, A., Williams, M.L., Akimoto, H., Amann, M., Baklanov, A., Baltensperger, U., Bey, I., Blake, N., Blake, R.S., Carslaw, K., Cooper, O.R., Dentener, F., Fowler, D., Fragkou, E., Frost, G.J., Generoso, S., Ginoux, P., Grewe, V., Guenther, A., Hansson, H.C., Henne, S., Hjorth, J., Hofzumahaus, A., Huntrieser, H., Isaksen, I.S.A., Jenkin, M.E., Kaiser, J., Kanakidou, M., Klimont, Z., Kulmala, M., Laj, P., Lawrence., M.G., Lee, J.D., Liousse, C., Maione, M., McFiggans, G., Metzger, A., Mieville, A., Moussiopoulos, N., Orlando, J.J., O’Dowd, C.D., Palmer, P.I., Parrish, D.D., Petzold, A., Platt, U., Pöschl, U., Prévôt, A.S.H., Reeves, C.E., Reimann, S., Rudich, Y., Sellegri, K., Steinbrecher, R., Simpson, D., ten Brink, H., Theloke, J., van der Werf, G.R., Vautard, R., Vestreng, V., Vlachokostas, Ch., von Glasow, R., (2009). Atmospheric composition change - global and regional air quality. Atmospheric Environment 43 (33), 5268-5350. 
Nicole, B. \& Frank, F. (eds.). (2004). Mini MAX-DOAS - an Introduction. Heidelberg, Germany: Institute of Environmental Physics, University of Heidelberg.

Ortega, I., Koenig, T., Sinreich, R., Thomson, D., and Volkamer, R. (2015). The CU 2-D-MAXDOAS instrument - Part 1: Retrieval of 3-D distributions of $\mathrm{NO}_{2}$ and azimuth-dependent OVOC ratios, Atmos. Meas. Tech., 8, 2371-2395, https://doi.org/10.5194/amt-8-23712015, 2015.

Palmer, P.I., Jacob, D.J., Fiore, A.M., Martin, R.V., Kelly, C., Kurosu, T.P. (2003). Mapping isoprene emissions over North America using formaldehyde column observations from space. J. Geophys. Res.: Atmosphere, 108.

Perner, D., Platt, U. (1979). Detection of nitrous acid in the atmosphere by differential optical absorption. Geophys. Res. Lett. 6 (12), 917-920.

Peters, E., Wittrock, F., GroBmann, K., FrieB, U., Richter, A., Burrows, J. (2012). Formaldehyde and nitrogen dioxide over the remote western Pacific Ocean: SCIAMACHY and GOME-2 validation using ship- based MAX-DOAS observations. Atmos. Chem. Phys. 12 (22), 11179.

Picornell, M., Ruiz, T., Borge, R., Garcia-Albertos, P., de la Paz, D., Lumbreras, J. (2019). Population dynamics based on mobile phone data to improve air pollution exposure assessments. J Expo Sci Environ Epidemiol 29, 278-291. https://doi.org/10.1038/s41370018-0058-5.

Pinardi, G., Van Roozendael, M., Abuhassan, N., Adams, C., Cede, A., Clémer, K., Fayt, C., FrieB, U., Gil, M., Herman, J., Hermans, C., Hendrick, F., Irie, H., Merlaud, A., NavarroComas, M., Peters, E., Piters, A.J.M., Puentedura, O., Richter, A., Schönhardt, A., Shayganfar, R., Spinei, E., Strong, K., Takashima, H., Vrekoussis, M., Wagner, T., Wittrock, 
F., Yilmaz, S. (2013). MAX-DOAS formaldehyde slant column measurements during CINDI: intercomparison and analysis improvement. Atmospheric Measurement Technique 6, 167185.

Plane, J.M.C., Saiz-Lopez, A. (2006). UV-Visible Differential Optical Absorption Spectroscopy (DOAS). In: Heard, D.E. (Ed.), Analytical Techniques for Atmospheric Measurement. Blackwell Publishing, Oxford.

Platt, U. (1994). Differential optical absorption spectroscopy (DOAS). In: Air Monitoring by Spectroscopic Techniques", Chem. Anal. Ser, vol. 127 M. W. Sigrist.

Platt, U., Stutz, J. (2008). Differential Optical Absorption Spectroscopy: Principles and Applications. Springer Berlin Heidelberg, Berlin, Heidelberg, Berling, Heidelberg.

Possanzini, M., Di Palo, V., Petricca, M., Fratarcangeli, R., Brocco, D. (1996). Measurements of lower carbonyls in Rome ambient air. Atmos. Environ. 30 (22), 3757-3764.

Prados-Roman, C., Cuevas, C.A., Hay, T., Fernandez, R.P., Mahajan, A.S., Royer, S.-J., Galí, M., Simó, R., Dachs, J., Großmann, K., Kinnison, D.E., Lamarque, J.-F., Saiz-Lopez, A. (2015). lodine oxide in the global marine boundary layer. Atmos. Chem.Phys. 15, 583-593.

Quaassdorff C., Borge R., Pérez J., Lumbreras J., de la Paz D., de Andrés J.M. (2016). Microscale traffic simulation and emission estimation in a heavily trafficked roundabout in Madrid (Spain). Sci Total Environ, 566-567:416-427. doi:10.1016/j.scitotenv.2016.05.051.

Richter, A., Burrows, J.P., NüB, Hendrick, Granier, C., Niemeier, U. (2005). Increase in tropospheric nitrogen dioxide over China observed from space. Nature 437 (7055), 129. 
Rodgers, C.D. (2000). Inverse Methods for Atmospheric Sounding: Theory and Practice. World Scientific Publishing, Singapore (Singapore).

Rothman, L.S., Gordon, I.E., Barber, R.J., Dothe, H., Gamache, R.R., Goldman, A., Perevalov, V.L., Tashkum, S.A., Tennyson, J. (2010). HITEMP, the high-temperature molecular spectroscopic database. J. Quant. Spectrosc. Radiat. Transf. 111 (15), 2139, 2150.

Sanders, R. W., Solomon, S., Smith, J. P., Perliski, L., Miller, H.L., Mount, G. H., Keys, J. G., and Schmeltekopf, A. L. (1993). Visible and Near-Ultraviolet Spectroscopy at McMurdo Station Antarc-tica, 9. Observations of OClO from April to October 1991, J.Geophys. Res., 98, D4, 7219-7228.

Saiz-Lopez, A., Shillito, J. A., Coe, H., and Plane, J. M. C. (2006). Measurements and modelling of $\mathrm{I}_{2}, \mathrm{IO}, \mathrm{OIO}, \mathrm{BrO}$ and $\mathrm{NO}_{3}$ in the mid-latitude marine boundary layer, Atmos. Chem. Phys., 6, 1513-1528.

Saiz-Lopez, A., Borge, R., Notario, A., Adame, J.A., de la Paz, D., Querol, X., Artiñano, B., Gómez-Moreno, F.J., Cuevas, C.A. (2017). Unexpected Increase in the Oxidation Capacity of the Urban Atmosphere of Madrid, Spain. Scientific Reports 7 (45956).

Serdyuchenki, A., Gorshelev, V., Weber, M., Chehade, W., Burrows, J.P. (2014). High spectral resolution ozone absorption cross-sections - Part 2: temperature dependence. Atmospheric Measurement Techniques 7 (2), 625-636.

Sin, D.W.M., Wong, Y., Louie, P.K.K. (2001). Trends of ambient carbonyl compounds in the urban environment of Hong Kong. Atmos. Environ. 35 (34), 5961-5969. 
Sinreich, R., Filsinger, F., Friess, U., Platt, U., Sebastian, O., Wagner, T., Volkamer, R., Kern, C. (2007). MAX-DOAS detection of glyoxal during ICARTT 2004. Atmos. Chem. Phys. 7, 12931303.

Sinreich, R., Coburn, S., Dix, B., Volkamer, R. (2010). Ship-based detection of glyoxal over the remote tropical Pacific Ocean. Atmos. Chem. Phys. 10, 11359-11371.

Schreier, S. F., Richter, A., and Burrows, J. P. (2019). Near-surface and path-averaged mixing ratios of $\mathrm{NO}_{2}$ derived from car DOAS zenith-sky and tower DOAS off-axis measurements in Vienna: a case study, Atmos. Chem. Phys., 19, 5853-5879, https://doi.org/10.5194/acp-195853-2019.

Solomon, S., Schmeltekopf, A.L., Sanders, R.W. (1987). On the interpretation of zenith sky absorption measurements. Journal of Geophysical Research 92, 8311-8319 Series C. Oceans and atmospheres, 1987.

Stamnes K, Tsay SC, Wiscombe W, Jayaweera K. (1988). Numerically stable algorithm for discrete-ordinate-method radiative transfer in multiple scattering and emitting layered media. Appl Opt. Jun 15;27(12):2502-9. doi: 10.1364/AO.27.002502.

Stutz, J., Kim, E.S., Platt, U., Bruno, P., Perrino, C., Febo, A. (2000). UV-visible absorption cross sections of nitrous acid. J. Geophys. Res.-Atmos. 105, 14585-14592.

Thalman, R., Volkamer, R. (2013). Temperature dependent absorption cross-sections of O2$\mathrm{O} 2$ collision pairs between 340 and $630 \mathrm{~nm}$ and at atmospherically relevant pressure. Phys. Chem. Chem. Phys. 15 (37), 15371-15381.

Vandaele, A.C., Hermans, C., Simon, P.C., Carleer, M., Colin, R., Fally, S., Mérienne, M.F., Jenouvrier, A., Coquart, B., (1998). Measurements of the NO2 absorption cross-section 
from $42000 \mathrm{~cm}^{-1}$ to $10000 \mathrm{~cm}^{-2}(238-1000 \mathrm{~nm})$ at $220 \mathrm{~K}$ and $294 \mathrm{~K}$. Journal of Quantitative Spectroscopy and Radiative Transfer; Atmospheric Spectroscopy Application 59 (3), 17118496.

Vigouroux, C., Hendrick, F., Stavrakou, T., Dils, B., De Smedt, I., Hermans, C., Merlaud, A., Scolas, F., Senten, C., Vanhaelewyn, G., Fally, S., Carleer, M., Metzger, J.-M., Müller, J.-F., Van Roozendael, M., De Mazière, M. (2009). Ground-based FTIR and MAX-DOAS observations of formaldehyde at Réunion Island and comparisons with satellite and model data. Atmos. Chem. Phys. 9, 9523-9544.

Volkamer, R., Molina, L.T., Molina, M.J., Shirley, T., Brune, W.H. (2005a). DOAS measurements of glyoxal as an indicator for fast VOC chemistry in urban air. Geophys. Res. Lett. 32 (8).

Volkamer, R., Spietz, P., Burrows, J., Platt, U. (2005b). High-resolution absorption crosssection of glyoxal in the UV-vis and IR spectral ranges. J. Photochem. Photobiol. Chem. 172 (1), 35-46.

Volkamer, R., Sheehy, P.M., Molina, L.T., Molina, M.J. (2007). Oxidative capacity of the Mexico City atmosphere - part 1: a radical source perspective. Atmos. Chem. Phys. Discuss. 7 (2), 5365-5412.

Vrekoussis, M., Wittrock, F., Richter, A., Burrows, J. (2010). GOME-2 observations of oxygenated VOCs: what can we learn from the ratio glyoxal to formaldehyde on a global scale? Atmos. Chem. Phys. 10 (21), 10145.

Wagner, T., Von Friedeburg, C., Wenig, M., Otten, C., Platt, U. (2002). UV-visible observations of atmospheric $\mathrm{O} 4$ absorptions using direct moonlight and zenith-scattered 
sunlight for clear-sky and cloudy sky conditions. J. Geophys. Res. Atmos. 107 pp. AAC 3-1; AAC 3-15.

Wagner, T., Dix, B., Friedeburg, C.v., FrieB, U., Sanghavi, S., Sinreich, R., Platt, U. (2004). MAX-DOAS O4 measurements: a new technique to derive information on atmospheric aerosols-principles and information content. J. Geophys. Res.: Atmosphere 109, D22.

Wagner, T., Deutschmann, T., Platt, U. (2009). Determination of aerosol properties from MAX-DOAS observations of the Ring effect. Atmos. Meas. Tech. 2 (2), 495-512.

Wagner, T. \& Beirle, S. \& Benavent, N. \& Bösch, T. \& Lok Chan, K. \& Donner, S. \& Dörner, S. \& Fayt, C. \& Frieß, U. \& García-Nieto, D. \& Gielen, C. \& González-Bartolome, D. \& Gomez Martin, L. \& Hendrick, F. \& Henzing, B. \& Li Jin, J. \& Lampel, J. \& Ma, J. \& Mies, K. \& Yela, M. (2018). Is a scaling factor required to obtain closure between measured and modelled atmospheric $\mathrm{O}_{4}$ absorptions? - A case study for two days during the MADCAT campaign. Atmospheric Measurement Techniques Discussions. 1-85. 10.5194/amt-2018-238.

Wang, S., Cuevas, C.A., Frieß, U., Saiz-Lopez, A. (2016). MAX-DOAS retrieval of aerosol extinction properties in Madrid, Spain. Atmospheric Measurement Techniques 9, 50895101.

Wang, Y., Beirle, S., Hendrick, F., Hilboll, A., Jin, J., Kiuberis, A.A., Lampel, J., Li, A., Luo, Y., Lodi, L., Ma, J., Navarro, M., Ortega, I., Peters, E., Polyansky, O.L., Remmers, J., Richter, A., Puentedura, O., Van Roozendael, M., Seyler, A., Tennyson, J., Volkamer, R., Xie, P., Zobov, N.F., Wagner, T. (2017). MAX-DOAS measurements of HONO slant column densities during the MAD-CAT campaign: inter-comparison, sensitivity studies on spectral analysis settings, and error budget. Atmos. Meas. Tech. 10, 3719-3742. 
Wedderburn, R. W. M. (1974). Quasi-likelihood functions, generalized linear models, and the Gauss-Newton method, Biometrika, Volume 61, Issue 3, Pages 439-447, https://doi.org/10.1093/biomet/61.3.439

Wojtal, P., Halla, J.D., McLaren, R. (2011). Pseudo steady states of HONO measured in the nocturnal marine boundary layer: a conceptual model for HONO formation on aqueous surfaces. Atmos. Chem. Phys. 11 (7), 3243-3261.

Widenhorn, R- \& Blouke, M. \& Weber, A. \& Rest, A. \& Bodegom, E. (2002). Temperature dependence of dark current in a CCD. Proceedings of SPIE - The International Society for Optical Engineering. 4669. 10.1117/12.463446.

World Health Organization. (2019). World health statistics 2019: monitoring health for the SDGs, sustainable development goals. World Health Organization. https://apps.who.int/iris/handle/10665/324835. License: CC BY-NC-SA 3.0 IGO

Zhang, L., Wang, T., Zhang, Q., Zheng, J., Xu, Z., Lv, M. (2016). Potential sources of nitrous acid (HONO) and their impacts on ozone: a WRF-Chem study in a polluted subtropical region. J. Geophys. Res. Atmos. 121 (7), 3645-3662. 